\title{
The Sensitivity of Wellbeing to Inequalities in Local Wellbeing
}

\author{
Pascarn Ronald Dickinson
}

A thesis submitted to Victoria University of Wellington in partial fulfilment of requirements for the degree of Master of Science

2018

School of Geography, Environment and Earth Sciences

Victoria University of Wellington 



\section{Abstract}

Research points to a robust negative relationship between average levels of subjective wellbeing and the distribution of subjective wellbeing. The fact that our wellbeing falls as wellbeing distributions widen suggests we care about inequalities in the lives of others. Central to this relationship is the role of place and human geography. The literature relating wellbeing to inequality in wellbeing is confined almost exclusively to inter-country comparisons. Virtually no attention has been paid to the relationship between wellbeing and inequality within countries - at the level of regions and below. The aim of this thesis is to test the generality of the inter-country evidence in the sub-national context.

I present four hypotheses which I test on three separate cross-sectional surveys: the New Zealand Quality of Life Survey, The New Zealand General Social Survey and the survey of Māori wellbeing, Te Kupenga. I follow the literature in using the standard deviation of wellbeing as a measure of wellbeing inequality. In each case the negative relationship between individual wellbeing and wellbeing inequality is clearly identifiable. The wellbeing effect of living in a place one standard deviation higher than another is roughly equivalent to the difference between the wellbeing of someone who is fully employed and someone who is unemployed and looking for work. Clearly we are highly sensitive to disparities in the subjective wellbeing of those around us.

I conduct several tests of the psychological drivers that lie behind the wellbeing response to local inequality in wellbeing. The first tests fairness perceptions, and finds sensitivity to wellbeing inequality to be higher among those who do not believe society is intrinsically fair. My test of altruism, while not as convincing empirically, suggests altruistic people may also be less affected by local wellbeing inequality. Both conclusions are consistent with the implied causation running from inequality to wellbeing.

While an important addition to the wellbeing literature, the more important implication of my findings is political. As the New Zealand Local Government Act comes up for renewal, the evidence I have assembled strongly supports providing local government with a clear purpose and the necessary funding to address the underlying causes of local inequalities in wellbeing. On empirical grounds alone, reducing wellbeing inequality is likely to make us all much happier. 
ii | P a g e

\section{Acknowledgements}

Access to some of the data used in this study was provided by Statistics New Zealand under conditions designed to give effect to the security and confidentiality provisions of the Statistics Act 1975. I am grateful to both Statistics New Zealand and the Quality of Life Project for the survey data, without whom this thesis would not be possible.

I cannot thank my supervisor Philip Morrison enough. I could not have produced this thesis without your knowledge and support. Your advice has played a key role in my learning over the past year and has encouraged me to think more in depth than I ever have before.

To all my fellow postgrads, thank you for your encouragement and company. If I did not have such a kind and supportive network, this year would have been much harder.

Finally, my thanks go to both Eleanor Wenman and Kealagh Robinson for their kindness (and proof reading) over the past year. Your support has ensured that while I focus on researching the wellbeing of others, my own wellbeing has been protected too. 
iii | P a g e

\section{Contents}

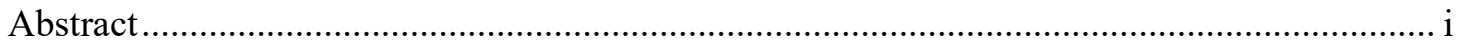

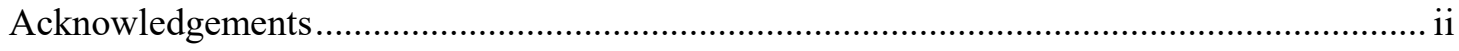

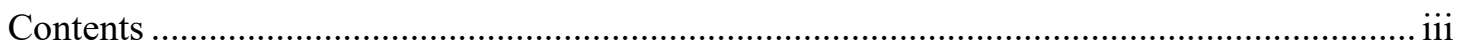

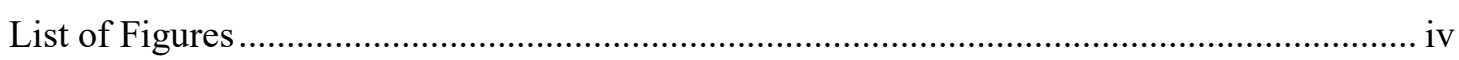

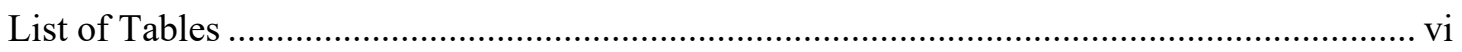

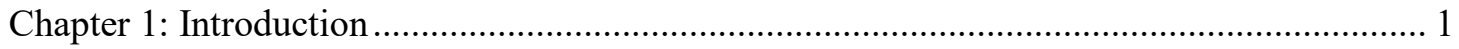

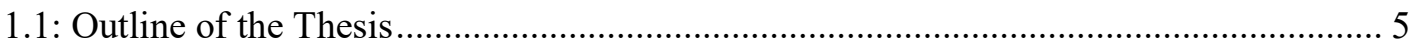

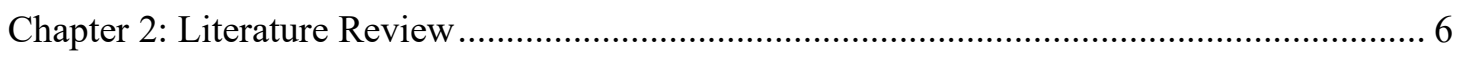

2.1: Income Inequality and Subjective Wellbeing .......................................................... 7

2.2: How do Income Inequality and Subjective Wellbeing Inequality Differ?..................... 9

2.3: What Drives the Sensitivity of Our Wellbeing to Wellbeing Inequality? ..................... 11

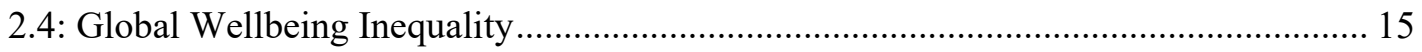

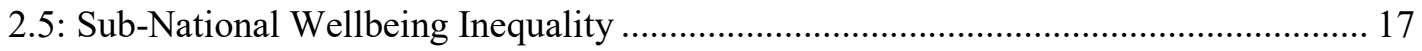

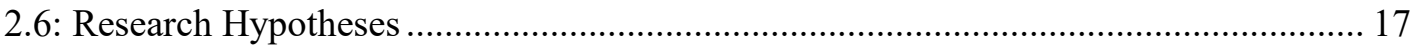

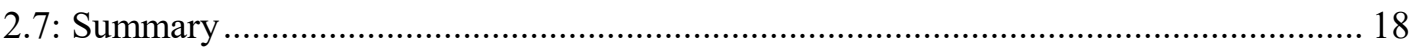

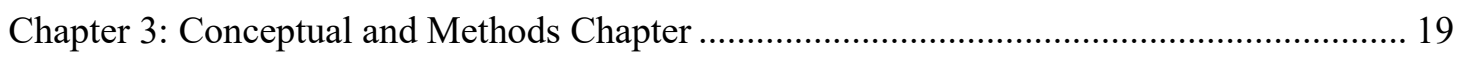

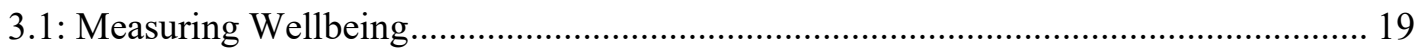

3.2: What is the Relationship Between Wellbeing and Wellbeing Inequality? .................. 20

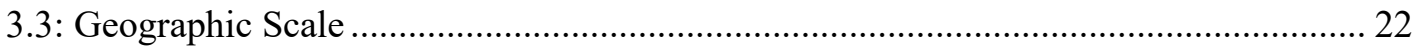

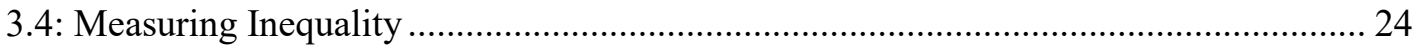

3.5: Modelling the Wellbeing and Wellbeing Inequality Relationship................................ 25

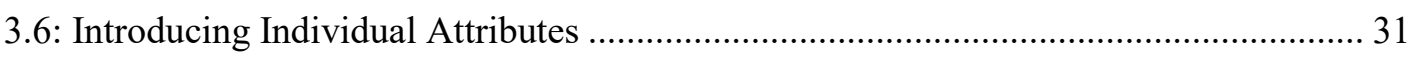

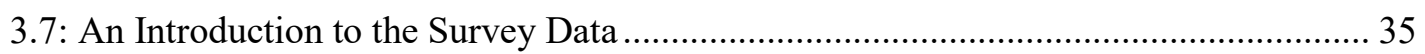

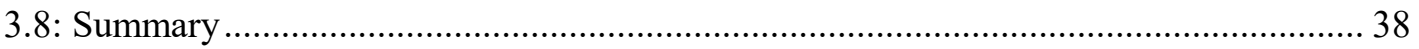

Chapter 4: Are People Sensitive to Wellbeing Inequality Where They Live?.......................... 40

4.1: The Primary Hypothesis - Does Average Wellbeing Decline

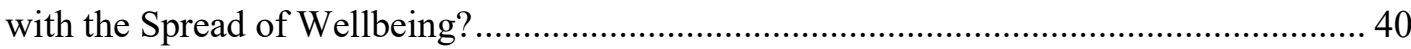

4.2: The Primary Hypothesis - Does Individual Wellbeing Decline

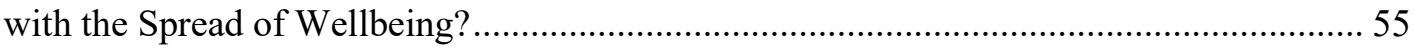

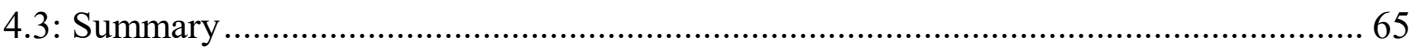

Chapter 5: What Mechanisms Drive Sensitivity to Wellbeing Inequality? ............................... 66

5.1: The Primary Hypothesis - Confirming Wellbeing is Sensitive

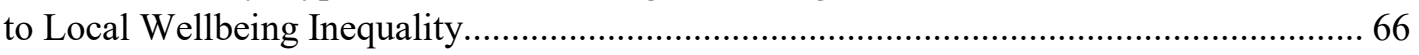

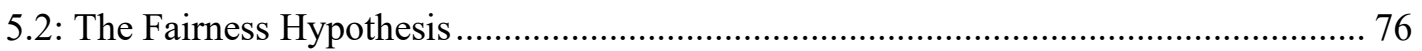

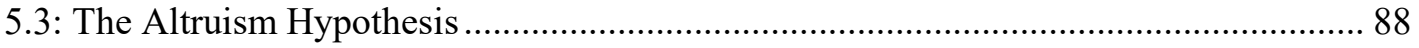

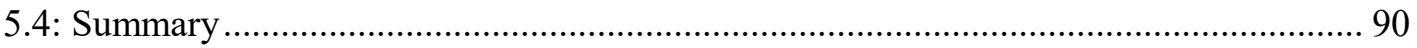

Chapter 6: Do These Mechanisms Apply for Māori? ............................................................... 92 
6.1: The Primary Hypothesis - Does the Wellbeing of Māori Decline with the Inequality of Wellbeing Among Māori? ..................................................................93

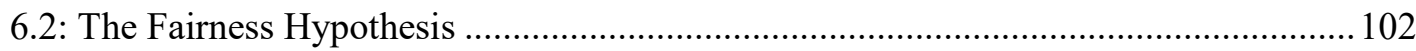

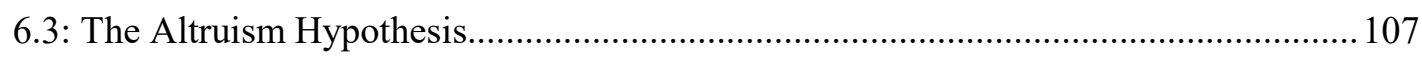

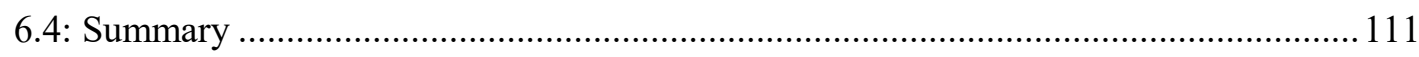

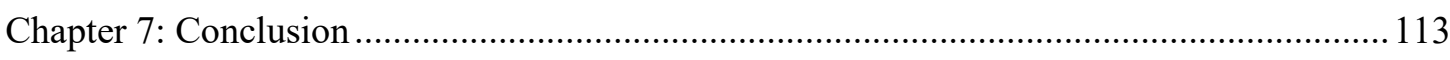

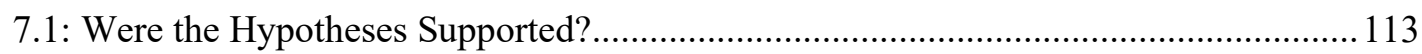

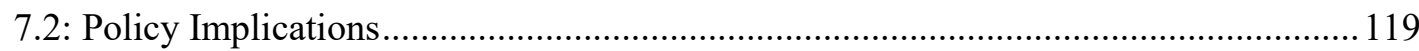

7.3: Limitations and Recommendations for Future Research ........................................... 120

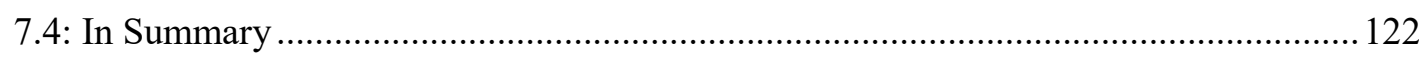

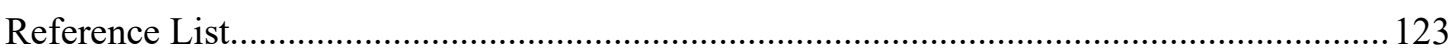

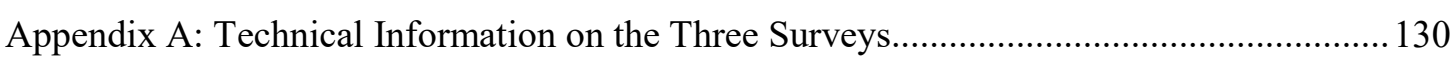

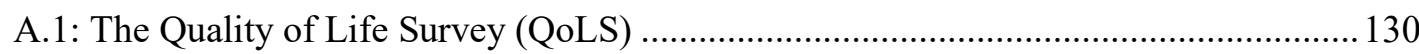

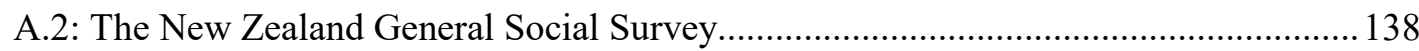

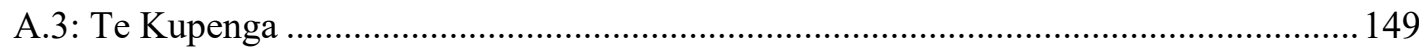

\section{List of Figures}

Figure 3.1: Four examples of inter-country negative relationships

between wellbeing and wellbeing inequality.

Figure 3.2: Sensitivity of average wellbeing to wellbeing inequality

at different scales

Figure 3.3: The theoretical sensitivity of wellbeing to wellbeing inequality at different sub-national scales

Figure 3.4: Histogram of life satisfaction in the Quality of Life Survey

for 2010 and 2012

Figure 3.5: Histogram of life satisfaction in the New Zealand General

Social Survey for 2008, 2010, and 2012

Figure 3.6: Histogram of life satisfaction in Te Kupenga

Figure 3.7: Histogram of the standard deviation life satisfaction of wards in the quality of life survey

Figure 3.8: A visual representation of the factors likely to influence sensitivity to wellbeing inequality

Figure 4.1: The relationship between mean and standard deviation life satisfaction by selected cities.

Figure 4.2: The relationship between mean and standard deviation life satisfaction among Auckland wards

Figure 4.3: Map of Auckland wards available for analysis in the Quality of Life Survey

Figure 4.4: The relationship between mean and standard deviation life satisfaction among Wellington Region wards 
$\mathbf{v}$ | P a g e

Figure 4.5: Map of Wellington Region wards available for analysis in the Quality of Life Survey

Figure 4.6: The relationship between mean and standard deviation life satisfaction among Christchurch wards

Figure 4.7: Map of Christchurch wards available for analysis in the Quality of Life Survey

Figure 4.8: The relationship between mean and standard deviation

life satisfaction among Dunedin wards

Figure 4.9: Map of Dunedin wards available for analysis in the Quality of Life Survey

Figure 4.10: The relationship between mean and standard deviation life satisfaction among New Zealand wards.

Figure 4.11: The relationship between mean and standard deviation life satisfaction among New Zealand wards, 2012 and 2014.

Figure 4.12: The relationship between mean and standard deviation life satisfaction by selected cities, 2012 and 2014

Figure 4.13: The relationship between mean and standard deviation life satisfaction among Auckland wards, 2012 and 2014.

Figure 4.14: The relationship between mean and standard deviation life satisfaction among Wellington Region wards, 2012 and 2014

Figure 4.15: The relationship between mean and standard deviation life satisfaction among Christchurch wards, 2012 and 2014

Figure 4.16: The relationship between mean and standard deviation life satisfaction among Dunedin wards, 2012 and 2014...

Figure 4.17: The spread of standard deviation life satisfaction by year

Figure 4.18: Coefficient plot of standard deviation life satisfaction and controls on individual life satisfaction in 2012

Figure 4.19: Coefficient plot of standard deviation life satisfaction and controls on individual life satisfaction in 2014

Figure 5.1: The relationship between mean and standard deviation life satisfaction by region

Figure 5.2: The relationship between mean and standard deviation life satisfaction by urban-region

Figure 5.3: Coefficient plot of standard deviation life satisfaction and controls on individual life satisfaction in 2012

Figure 5.4: Predicted individual life satisfaction by standard deviation life satisfaction by perceptions of fairness, no controls

Figure 5.5: Predicted individual life satisfaction by standard deviation life satisfaction by perceptions of fairness, with controls

Figure 5.6: Predicted individual life satisfaction by standard deviation life satisfaction by perceptions of fairness, high deprivation areas.

Figure 6.1: The relationship between mean and standard deviation life satisfaction by New Zealand regions 
vi | P a g e

Figure 6.2: The relationship between mean and standard deviation life satisfaction by urban-region categories

Figure 6.3: The frequency weighted relationship between mean and standard deviation life satisfaction by urban-region categories .97

Figure 6.4: Coefficient plot of standard deviation life satisfaction and controls on individual life satisfaction

Figure 6.5: Predicted individual life satisfaction by standard deviation

life satisfaction by perceptions of fairness, no controls

Figure 6.6: Predicted individual life satisfaction by standard deviation

life satisfaction by trust in others, no controls

Figure 6.7: Predicted individual life satisfaction by standard deviation

life satisfaction by community help provided, no controls

Figure 6.8: Predicted individual life satisfaction by standard deviation life satisfaction by housework help provided to those outside of the household, no controls

\section{List of Tables}

Table 3.1: Number of responses to the life satisfaction question within each survey by year.....

Table 3.2: Summary statistics on key control variables as they appear in each of the three surveys

Table 4.1: Models of individual life satisfaction against standard deviation life satisfaction of wards, 2012 (cluster-controlled) .56

Table 4.2: Models of individual life satisfaction against standard deviation life satisfaction of wards, 2014 (cluster-controlled)

Table 5.1: Details on the NZGSS urban-region variable

Table 5.2: Models of individual life satisfaction against standard deviation life satisfaction of urban-region group, 2012 (cluster-controlled)

Table 5.3: OLS regression of life satisfaction on the interaction between perceptions of fairness and standard deviation life satisfaction for 2008 80

Table 6.1: Details for the Te Kupenga urban-region variable 94

Table 6.2: Models of individual life satisfaction against standard deviation life satisfaction of urban-region group, 2013 (cluster-controlled) 


\section{Chapter 1: Introduction}

This thesis builds on a 40 year enquiry into the reasons for variation in wellbeing. Easterlin (1974) prompted the rapid growth of the economics literature on wellbeing with his investigation of the relationship between wellbeing and income. Others followed, seeking to explore the relationship between wellbeing and the income of others, rather than just our own (Katic \& Ingram, 2017; Oishi, Kesebir \& Diener, 2011; Wilkinson \& Pickett, 2009). More recently, research has addressed the way that the wellbeing of others effects our own wellbeing, focusing on the relationship between wellbeing and wellbeing inequality across nations (Goff, Helliwell \& Mayraz, 2016; Ott, 2005). I take the next step in this inquiry by examining the relationship between wellbeing and wellbeing inequality within countries.

Wellbeing in this context refers to subjective wellbeing (SWB), as opposed to objective measures of wellbeing such as health or income. As Quick (2015) explains:

"Wellbeing can be defined as the extent to which an individual or group experiences their life as going well, based on experiencing positive emotions, functioning well and meeting basic psychological needs" (p. 7).

The most common measure of SWB within the literature is life satisfaction. The life satisfaction question from the New Zealand Quality of Life Survey is typical of most: "Taking everything into account, how satisfied or dissatisfied are you with your life in general these days?" on a scale of 1 'very dissatisfied' to 5 'very satisfied' .

But why should politicians and the academy care about wellbeing? You likely already know one reason innately; that SWB in the form of happiness and satisfaction is what we all strive for. As Layard (2016) argues:

"if we ask why it matters how people feel - why happiness is good - we can give no answer. It is just self-evident. So happiness is revealed as the overarching good, and other goods obtain their goodness from the fact that they contribute to happiness." (p. 52).

\footnotetext{
${ }^{1}$ Throughout this thesis I use the terms 'wellbeing' and 'SWB' interchangeably. Where relevant, I will refer to a specific measure of SWB, such as 'happiness' or 'life satisfaction'.
} 
However, there are more academic reasons as to why wellbeing deserves our attention as well. Perhaps the main attraction of SWB measures from a policy perspective is that they are indicators of people's evaluations of life as a whole. Veenhoven (2005) argues that objective measures such as Gross Domestic Product (GDP) or GDP per capita are reasonably effective measures of life-chances, but that SWB measures can give us unparalleled insight into people's life-results.

As a result, researchers have advocated the use of SWB measures as more effective indicators of progress than GDP or GDP per capita, and have encouraged their widespread adoption in governance (Clark, Frijters \& Shields, 2008; Easterlin, 1974; Helliwell, Layard \& Sachs, 2013). It is therefore a natural progression of this argument that SWB inequality (i.e. the distribution of SWB) is a more sensitive measure of unequal development progress than income inequality (Goff et al., 2016; Helliwell, Huang \& Wang, 2016; Kalmijn \& Veenhoven, 2014; Quick, 2015; Veenhoven \& Kalmijn, 2005).

If SWB has been shown to be a better measure of progress than income or GDP, why isn't SWB more widely used? The primary reason is that people tend to confuse income with happiness. Many contemporary economists, politicians and members of the public consider income to be synonymous with wellbeing. While some governments have committed to integrating SWB into their approach to governance (e.g. Bhutan, see Ura, Alkire \& Zangmo, 2012) much of the world has been slow to change, and progress as measured by economic growth is still a dominant part of political discourse internationally.

In spite of the relative inefficacy of income inequality as a development measure, the literature on income inequality has blossomed in recent decades. The most notable work is Wilkinson and Pickett's influential book The Spirit Level (2009), in which the authors conclude that income inequality is harmful to our wellbeing in a number of ways. The SWB literature has also long been interested in the impacts of income inequality on wellbeing (Graham \& Felton, 2006; Kelley \& Evans, 2017; Ngamaba, Panagioti \& Armitage, 2018; Oishi, Kesebir \& Diener, 2011; Tomes, 1986). While results are inconclusive, in developed nations income inequality is generally found to be negative for our wellbeing (Kelley \& Evans, 2017). However, research has largely focused on levels of wellbeing, while interest in wellbeing inequality remains lacklustre (Quick, 2015). 
Debate about whether individuals desire the greatest happiness for the greatest number (i.e. are 'utilitarian') or desire equality in happiness ('egalitarian') dates back centuries (Bentham, 1823; Hutcheson, 1726; Mill, 1863; Paine, 1792). It is only with the recent availability of survey datasets with large sample sizes that we have been in a position to actually document the distribution of people's SWB. We are now able to empirically test whether levels of wellbeing in a community may increase or decrease with the dispersion of wellbeing in that community. In spite of these tests having been feasible for decades, measuring SWB inequality is a relatively recent practice (Kalmijn \& Veenhoven, 2005).

However, a small but growing literature base does examine the link between levels of wellbeing and dispersion of wellbeing, and finds a negative relationship between wellbeing and wellbeing inequality at the international level (Delhey, 2004; Fahey \& Smyth, 2004; Goff et al., 2016; Ott, 2005). In other words, as wellbeing inequality in a country rises, levels of wellbeing in that country tend to decline. Such variations in wellbeing inequality are predominantly explained using country-level institutional factors such as governance or levels of economic growth (Abdallah \& Quick, 2016; Helliwell et al., 2016; Ott, 2005; Ovaska \& Takashima, 2010; Veenhoven, 2005).

In contrast, research on wellbeing inequality at the sub-national level is in its infancy. The one study that does find a negative relationship between wellbeing and wellbeing inequality offers no mechanisms to explain it (Tesfazghi, Martinez \& Verplanke, 2010). Given that country-level effects are held constant for individuals and groups within the same nation, the institutional mechanisms used to explain the international relationship cannot simply be applied to the sub-national relationship. As such, other drivers of sensitivity to SWB inequality need to be investigated.

We know New Zealanders differ markedly in their preferences for greater or reduced inequality (Morrison, 2015a). However, the attitudes of New Zealanders to wellbeing inequality are unexplored, as are the possible effects of that inequality. Do people in New Zealand care about inequalities in wellbeing? Is their own wellbeing affected by those inequalities?

In this thesis, I document and explain the relationship between wellbeing and wellbeing inequality at various scales within New Zealand. On the basis of previous research, I predict that New Zealanders will demonstrate lower subjective wellbeing 
when they live in areas with a greater inequality in wellbeing. In other words, I expect that people will be less happy when there is greater spread in the happiness of people around them.

I then examine potential mechanisms driving the negative relationship between wellbeing and wellbeing inequality. Why are some people more sensitive to inequality than others? Drawing from the income inequality literature for explanatory mechanisms, I expect sensitivity to wellbeing inequality to be affected by the individual's outlook on society. Katic and Ingram (2017) present a strong case that those who perceive income inequality to be the result of a fair process face less of the negative SWB implications of that inequality. As such, I predict that the wellbeing of those who believe society treats individuals fairly will be less sensitive to wellbeing inequality than their more sceptical counterparts.

Research from psychology indicates that people with altruistic tendencies suffer more in the presence of inequality (Dawes, Fowler, Johnson, McElreath \& Smirnov, 2007; Johnson, Dawes, Fowler, McElreath \& Smirnov, 2009). As such, I also predict that people who are more altruistic will be more sensitive to wellbeing inequality than those who are more egoistic.

In summary, the primary arguments I make below are that levels of wellbeing fall as wellbeing inequality in an individual's local area rises, and that an individual's values dictate their sensitivity to that inequality. I am the first so far to test and explain this relationship at the sub-national level.

What might be the policy implications of a negative relationship between wellbeing and sub-national wellbeing inequality in New Zealand? Dalziel and Saunders (2014) contrast the Local Government Act (2002) put in place by Labour with the National Party amendment (2012). In 2002, one of the two statutory purposes of local government was defined as: "to promote the social, economic, environmental, and cultural wellbeing of communities, in the present and in the future" (Dalziel \& Saunders, 2014, p. 63, emphasis added). In 2012 this purpose was amended to: "to meet the current and future needs of communities for good quality local infrastructure, local public services, and performance of regulatory functions in a way that is most costeffective for households and businesses.” (Dalziel \& Saunders, 2014, p. 63). Evidently, with the National government came an explicit move away from prioritising wellbeing in communities, and towards prioritising finances. 
As such, if a negative relationship between wellbeing and wellbeing inequality at a regional level is established, it would have significant implications for the role of local government in improving the wellbeing of citizens. The policy implications of this research will be discussed in the conclusion.

\section{1: Outline of the Thesis}

This thesis consists of seven chapters, including this one. In Chapter 2, I review the literature on SWB and its relationship with inequality as measured in terms of income and wellbeing. The review is followed by the introduction of four research hypotheses.

In Chapter 3, I model the predicted relationship between SWB and SWB inequality and discuss the various forms these relationships can take. Chapter 3 also contains my discussion of the methods used in this thesis, including the measure of SWB inequality and individual attributes that will be controlled for in the analysis. The chapter concludes with a brief introduction to three New Zealand surveys.

Chapter 4 draws solely on the New Zealand Quality of Life Survey for 2012 and 2014, to comprehensively address the relationship between SWB and SWB inequality at both the city and ward level.

Chapter 5 draws on the New Zealand General Social Survey for 2008, 2010, and 2012 to confirm the negative relationship between wellbeing and wellbeing inequality at the sub-national level. I then test some of the potential explanatory mechanisms underlying the relationship, testing the impact of altruism and perceptions of fairness on individual sensitivity to SWB inequality.

Chapter 6 draws on Te Kupenga, the survey of Māori wellbeing conducted in 2013, to confirm the presence of a negative relationship between wellbeing and wellbeing inequality by using a culturally specific sub-set of the population. The impact of perceptions of fairness and altruism will also be re-tested in Chapter 6 .

The thesis concludes with Chapter 7, where I bring together the results from the three analysis chapters for discussion. I highlight the policy implications of my research and conclude with a discussion of questions for further research. 


\section{Chapter 2: Literature Review}

Philosophical discussion of the impact of societal wellbeing on individual happiness has been ongoing for centuries (Bentham, 1823; Mill, 1863). However, only relatively recently have academics had the measures, tools, and motivation to analyse and explain variations in wellbeing and their consequences.

The work of Easterlin (1974) is generally considered the catalyst for the rapid expansion of the SWB literature. Easterlin used data on 14 countries to demonstrate that at any point in time richer countries are happier than poorer countries, while over time countries do not get happier as they get richer (1974). This so called 'Easterlin Paradox' spurred a wave of new research into the relationship between income and SWB, with many attempting to explain the apparently counterintuitive findings Easterlin put forward (for a review of the income and SWB literature see Clark, Frijters \& Shields, 2008).

Economists also began to investigate other country-level characteristics. Some turned to income inequality as an explanation for differences in the average wellbeing of countries (Wilkinson \& Pickett, 2009). As a result, there has been a gradual acceptance within economics that while our SWB rises with our own income, our SWB is also determined in part by the income of others relative to our own (Clark et al., 2008).

From this attention to the income of others, questions surrounding the effect of the relative wellbeing of other people have arisen. Collectively, previous research into the way an individual's SWB is altered by other people's wellbeing has motivated the analysis of average wellbeing relative to inequality in wellbeing (Bolle, Okhrin \& Vogel, 2009; Delhey, 2004; Fahey \& Smyth, 2004; Helliwell et al., 2016).

The change in focus from the influence of income inequality on wellbeing to the influence of wellbeing inequality has also required a change in the proposed causal mechanism. Previously, primarily less desirable attributes (envy or pride) were used to explain the costs and benefits of possessing lower or higher relative income. Now, more positive attributes such as altruism, egalitarianism and compassion are used to explain our sensitivity to inequality (Katic \& Ingram, 2017).

The focus is no longer on upwards comparisons, but instead is on comparisons with the group as a whole. In a sense, the adoption of these new positive drivers has 
entailed a U-turn in the economic literature, back to those $18^{\text {th }}$ and $19^{\text {th }}$ century philosophers who pronounced virtuosity, compassion, and sympathy as the reasons that our happiness is affected by the happiness of others (Bentham, 1823; Mill, 1863).

Wellbeing inequality has primarily been addressed at the country-level (Becchetti, Massari \& Naticchioni, 2013; Chin-Hon-Foei, 1989; Delhey \& Kohler, 2011; Ifcher \& Zarghamee, 2016; Veenhoven, 2000; 2005). A smaller subset of research has specifically addressed the relationship between average wellbeing and wellbeing inequality, and has found a consistent negative relationship (Delhey, 2004; Fahey \& Smyth, 2004; Goff et al., 2016; Bolle et al., 2009).

The mechanisms underlying the relationship between wellbeing and wellbeing inequality are still relatively unexplored, but are generally attributed to country-level characteristics (Delhey, 2004; Ott, 2005). Only a few studies address SWB inequality within a nation (Becchetti et al., 2013; Dutta \& Foster, 2013; Stevenson \& Wolfers, 2008), and only one addresses the relationship between wellbeing and wellbeing inequality using sub-national spatial groupings (Tesfazghi et al., 2010). The research of Tesfazghi and colleagues (2010) indicates that country-level analyses may hide significant sub-national variations in SWB inequality.

Based on the international literature, I expect a negative relationship between wellbeing and wellbeing inequality at the sub-national level. However, country-level effects cannot explain sub-national differences in sensitivity to wellbeing inequality. In this thesis, I explore these gaps in our current knowledge.

I elaborate on the above points in six sections. In order to frame our thinking about the relationship between SWB and inequality, the first section reviews research on the relationship between SWB and income inequality (the most widely studied form of inequality). I then point out the differences between using income inequality and SWB inequality as measures of inequality. In section three I turn to the possible explanatory mechanisms underlying the relationship between wellbeing and wellbeing inequality. The fourth and fifth sections explore the current research on SWB inequality at the international and sub-national scales respectively. This chapter concludes with the research hypotheses to be addressed in the analysis.

\section{1: Income Inequality and Subjective Wellbeing}

While research on the relationship between SWB inequality and SWB is confined to only a handful of papers (Bolle et al., 2009; Delhey, 2004; Fahey \& Smyth, 
2004; Goff et al., 2016; Ott, 2005), the relationship between income inequality and SWB has been widely studied. The literature on income inequality and its relationship with SWB can therefore be useful for framing our thinking about the relationship between SWB and other forms of inequality, even if findings may not directly relate to the SWB and SWB inequality relationship. The literature primarily discusses income inequality in terms of the Gini coefficient, although other versions of Gini and alternative measures are also used (Ngamaba et al., 2018).

The literature is divided on the relationship between SWB and income inequality. A negative relationship has been identified by numerous researchers covering many countries and regions of the globe, using diverse datasets (Delhey \& Dragolov, 2014; Fahey \& Smyth, 2004; Goff et al., 2016; Graham \& Felton, 2006; Hagerty, 2000; Morawetz et al., 1977; Oishi et al., 2011; Schwarze \& Härpfer, 2007). However, other studies find either no relationship between SWB and income inequality (Helliwell, 2003; Senik, 2004), or a positive relationship (Haller \& Hadler, 2006; Katic \& Ingram, 2017; Tomes, 1986). For example, Kelley and Evans (2017) use data from 68 countries spanning over 20 years, and find that income inequality is positively related to wellbeing in developing nations, but find no evidence of a relationship in developed nations.

There is evidence of geographic differences in the relationship between income inequality and SWB of countries across the developed world as well. For instance, Alesina, Di Tella and MacCulloch (2004) find that while income inequality negatively impacts SWB in both the United States and Europe, the effect is stronger in Europe than in the United States. The authors attribute the relative lack of sensitivity to inequality in the United States to higher perceived social mobility (i.e. people are able to move up or down the distribution more easily), even though objective levels of social mobility in Europe and the United States are roughly equivalent. In doing so, they demonstrate that subjective perceptions of the nature of income inequality can mitigate the negative impacts of that inequality on our wellbeing (Alesina et al., 2004).

While low perceived social mobility is one mechanism suggested as underlying the negative effects of income inequality on SWB, other explanations have also been offered. Katic and Ingram (2017) draw on World Values Survey data for 99 countries to demonstrate that the relationship between SWB and income inequality is moderated by perceived unfairness, perceptions surrounding hard work leading to success, and the individual's own socioeconomic status. For example, those who perceived the income 
distribution as being due to an unfair process had lower wellbeing when income inequality was higher (Katic \& Ingram, 2017).

Similarly, Oishi and colleagues (2011) use General Social Survey data from the United States to show that the negative link between SWB and income inequality can be explained by perceived unfairness and low social trust. However, Delhey and Dragolov (2014) critique Oishi and colleagues (2011) by asserting that trust in others and perceptions of fairness are both measures of the same underlying value: 'faith in others'. Delhey and Dragolov (2014) find a negative relationship between SWB and income inequality within 30 European countries, and show that status anxiety explains the relationship in less affluent societies, while distrust explains the relationship in more affluent societies.

Taken together, evidence from analyses of the relationship between income inequality and SWB suggests a number of mechanisms to explain this relationship. However, could the mechanisms put forth to explain the relationship between wellbeing and income inequality also be used to explain the relationship between wellbeing and wellbeing inequality? Differences between these two measures must be considered before I can answer that question.

\section{2: How do Income Inequality and Subjective Wellbeing Inequality Differ?}

Wellbeing inequality has been found to operate differently from income inequality in the same locations. A consistent relationship between the two has not been found (for a meta-analysis of this literature, see Ngamaba et al., 2018).

For instance, Ovaska and Takashima (2010) use a dataset of 72 countries to compare income inequality and happiness inequality, and found that income inequality was one of the most significant drivers of happiness inequality. Delhey and Kohler (2011) also found a positive relationship between income inequality and rising SWB inequality, although their methodology has since been heavily critiqued for being inaccurate, with others finding no relationship when using the same dataset (Kalmijn, 2012; Veenhoven, 2012).

In contrast, Ott (2005) argues that decreasing SWB inequality could go alongside increasing income inequality, although does not test this theory using data. Stevenson and Wolfers (2008) go a step further, and find as income inequality in the United States increased between 1972 and 2006, happiness inequality actually decreased. 
Becchetti and colleagues (2013) reported on individual data from the German Socio-Economic Panel, and concluded that non-pecuniary factors are much more important than income inequality for driving happiness inequality. They concluded that income inequality could not be considered a driver of happiness inequality (Becchetti et al., 2013). Others have likewise found no significant relationship between income inequality and SWB inequality in 119 countries (Berg \& Veenhoven, 2010), and again for 33 European countries (Fahey \& Smyth, 2004).

Goff and colleagues (2016) aimed to determine which of the two measures of inequality best predicts wellbeing. They compared the impact of happiness inequality and income inequality on happiness in three international surveys and one large domestic survey from the United States. In all cases they found happiness inequality to have a stronger negative impact on happiness than income inequality.

It is also worth drawing attention to the work of Veenhoven (2000), who shows that state welfare (i.e. redistribution of income) neither reduces the level nor the inequality of happiness in 41 European nations. Veenhoven's work once again points towards the idea that addressing income inequality will not necessarily bring simultaneous benefits for wellbeing inequality.

There are several reasons why the empirical relationship between income inequality and SWB inequality may not be as close as one might at first imagine. Perhaps the most obvious is the distinction between income as a measure of life-chances and SWB as a measure of life-results (Veenhoven, 2005). While income represents the opportunities that an individual might have in life, SWB represents an individual's evaluation of their life overall. Income is a measure of how much people earn, but SWB is a measure of the life they live as a result of those earnings, as well as a measure of how well life is going in other domains.

Furthermore, while income is a finite resource, SWB is subject to no such restrictions. Given that the income available for distribution is finite at a given point in time, when we compete for income with others, it is a zero-sum game, as one must lose while another wins. In contrast, Layard (2016) refers to wellbeing as a 'positive-sum game', in that success for one brings success for others as well. Nobody is liable to feel they are losing out if SWB inequality is reduced, unlike when income inequality is reduced though redistribution.

I have demonstrated in this section that there is not necessarily a relationship between income inequality and wellbeing inequality. Therefore, it is possible the 
11 | P a g e

drivers of sensitivity to income inequality may not also drive sensitivity to wellbeing inequality. The following section will discuss the possible mechanisms underlying sensitivity to wellbeing inequality specifically.

\section{3: What Drives the Sensitivity of Our Wellbeing to Wellbeing Inequality?}

What is it that drives certain people to be more sensitive to inequality in the wellbeing of those around them? Why do some people appear to care more about others wellbeing? Do we care about all others, or just others 'like us'? Most researchers who have documented the link between the level and dispersion of SWB have not offered much in the way of explanation, beyond the level of wealth and institutional characteristics of the nation (Delhey, 2004; Fahey \& Smyth, 2004; Ott, 2005).

There are at least three compelling reasons why some individuals might be averse to wellbeing inequality: 1) we have evolved to care primarily about others in our in-group in order to survive; 2) we are willing to make self-sacrifices in order to encourage greater equality; and 3) some individuals perceive distribution in society to be less fair than others and are dissatisfied as a result. All of these explanatory mechanisms are related to the idea that some individuals are more caring or are more empathetic in general. Finally, it is important to note that the amount of inequality we are willing to tolerate before we become sensitive to it likely differs between (and even within) individuals.

\section{1: Those who cared about others close to them survived}

Evolutionary psychologists offer some useful insights as to why and to what degree humans prefer more equal social distributions. They consistently point out that humans have deep-rooted egalitarian preferences (Buss, 2000; Charlton, 1997; Dawes et al., 2007; Dawes et al., 2012; Fehr, Bernhard \& Rockenbach, 2008; Johnson et al., 2009).

Fehr and colleagues (2008) indicate that the inequality aversion of humans is the strongest within smaller groups. This preference for equality in the smaller group to which one belongs is known as 'parochialism' (Fehr et al., 2008). The more that the fate of a particular group is shared, the more that cooperation is enhanced, and the more averse people are to inequality (Buss, 2000). As a result, we are likely more averse to inequality at smaller, more localised scales such as the family or town than the nation as a whole. 
An important aside is that much of the literature on parochialism uses evidence from experiments where participants play income redistribution games (for examples, see Dawes et al., 2007; Fehr et al., 2008). These games vary in their intent, method, and specific structure, but generally consist of each participant being given a different amount of money, and having the option to engage in redistributive or greedy behaviour. Sometimes participants are given the option to punish others for their behaviour, and other times they are not.

As such, most evidence from such games is only indicative of people's sensitivity to income inequality. Even though parochialism could influence sensitivity to SWB inequality, there are likely differences between the way parochialism interacts with our sensitivities to income inequality as opposed to SWB inequality, especially given that SWB is not a resource which can be directly redistributed through any intervention.

While parochialism does help to explain why we might be sensitive to inequalities at smaller scales, it does not explain why we would still be sensitive to inequality in general. As such, it is likely that there is more than solely parochialism affecting our aversion to inequality.

\section{2: We are willing to make self-sacrifices to encourage equality}

Altruistic tendencies among people is another theoretical explanation for our sensitivity to inequality. When discussing altruism in the context of this theory, I refer not only to those wishing to help others worse off than themselves, but also to those who want to reduce the distribution overall by discouraging selfishness.

Generally, humans like helping people, and this leads to the redistribution of resources and greater equality (Dawes et al., 2007; Johnson et al., 2009). Johnson and colleagues (2009) found that egalitarian preferences underlie both altruistic punishment and cooperation in individuals. Altruistic punishment involves individuals spending their own resources in order to enforce greater equality (i.e. spending money to redistribute money from the greedy to the poor), and generally occurs in income games if individuals are given the power to do so (Johnson et al., 2009).

Dawes and colleagues (2007) also link altruism and egalitarianism. However, they go further to relate altruistic tendencies and inequality aversion to wellbeing by pointing out that as inequality rises, negative emotions also rise for people who are more altruistic. Dawes and colleagues (2012) later add validity to these findings by 
$\mathbf{1 3}$ | P a g e

demonstrating that the same neural basis underlies both egalitarian behaviour and participation in altruistic punishment. As such, we should expect that individuals with greater altruistic tendencies also have stronger egalitarian preferences, and therefore, a greater sensitivity to inequality.

Altruism as a trait is rarely measured directly in wellbeing surveys. However, we can still examine the effect of altruism by using a number of potentially closely related variables. For instance, females have been found to act in a more altruistic manner than males (Rand, Brescoll, Everett, Capraro \& Barcelo, 2016; Simmons \& Emanuele, 2007), and so we could expect females to be more sensitive to SWB inequality as a result of this altruism. Even better proxies of altruism could include the amount of support an individual offers to friends or strangers, measures of donations to charity, or frequency of participation in volunteer work.

At the same time, measures such as volunteering may capture motivations other than altruism (Becchetti, Corrado \& Conzo, 2017; Binder, 2015). For instance, Binder (2015) found that volunteering may play a protective role for individuals, preventing their SWB from being negatively impacted by dissatisfactory conditions. It is not unreasonable to believe such conditions may include SWB inequality. This means we may actually see a weaker relationship between SWB and SWB inequality for volunteers. Further, Becchetti and colleagues (2017) indicate that while volunteering is generally beneficial to wellbeing, altruistic values do not always underlie these actions.

While altruistic values may impact how inequality averse an individual is, their perceptions of that inequality may be just as important, if not more so.

\section{3: Some People Perceive Inequality to be Fairer than Others}

Perceived fairness is one of the explanatory mechanisms used in the literature to explain the relationship between income inequality and SWB (Delhey \& Dragolov, 2014; Katic \& Ingram, 2017; Oishi et al., 2011). For instance, Katic \& Ingram (2017) test whether those who perceive the income generation process as unfair are more likely to be negatively impacted by income inequality. They found a significant negative effect of inequality on SWB for those who perceive society as unfair, and a significant positive effect for those who perceived society to be fair.

Could this mechanism also help explain the relationship between an individual's SWB and SWB inequality? If an individual believes society is fair, they are less likely 
$14 \mid \mathrm{P}$ a g e

to be upset by inequality, because they will attribute it to poor luck or poor choices as opposed to societal injustice. In contrast, individuals who view societal systems as unfair are more likely to react negatively to higher inequality in wellbeing around them. There is some evidence of this in the SWB inequality literature, with one study finding that people who perceived income inequality as too high (similar to perceptions of fairness, but still distinct) were more negatively affected by wellbeing inequality in their country (Goff et al., 2016).

Perceptions of fairness in society can be measured by questions about respondents' views on society. Alternatively, measures of the trust that individuals have in other people can be used to indicate perceptions of fairness as well. An individual's ability to trust strangers to contribute to a fair society might be closely related to their evaluations of whether they perceive society to actually be fair or not (Delhey \& Dragolov, 2014). Likely related to both perceptions of fairness and altruism is how empathetic an individual is.

\section{$\underline{\text { Some People are More Empathetic than Others }}$}

Another factor potentially influencing sensitivity to SWB inequality is how caring or empathetic an individual is. Humans are generally good at being empathetic; at recognising and embodying the emotions of others (Davidson \& Schuyler, 2015). As a result, both positive and negative emotions show a tendency to spread through social networks (Davidson \& Schuyler, 2015; Fowler \& Christakis, 2008; Kramer, Guillory \& Hancock, 2014).

The development of empathy has been vital in encouraging pro-social activity among people, driving behaviours such as donating to charity or volunteering (Davidson \& Schuyler, 2015). How empathetic or caring an individual is may also impact their sensitivity to inequalities in SWB.

Unfortunately, empathy is rarely measured in surveys, and owing to the data available, I am unable to address it in the current research. As such, determining the influence of individual empathy on sensitivity to wellbeing inequality should be considered an avenue of exploration for future research.

\section{The Amount of Inequality Individuals are willing to Tolerate Differs}

Finally, when talking about sensitivity to SWB inequality, it is important to consider the likely magnitude of this sensitivity. It is doubtful that any individual, 
$\mathbf{1 5}$ | P a g e

regardless of how parochial or altruistic, would be deeply upset to see someone earn 1 cent more or less per year than them. Instead, it is likely we have a certain threshold of inequality that we deem acceptable, or 'equal enough'. It is when inequality increases above some implicit threshold that we react negatively.

Now that I have covered the theoretical mechanisms possibly underlying the sensitivity of wellbeing to wellbeing inequality, I will discuss the literature that has examined the empirical relationship between the two.

\section{4: Global Wellbeing Inequality}

The broadest scope analysis of SWB inequality at the global scale is a chapter in the World Happiness Report 2016 Update (Helliwell et al., 2016). Helliwell and colleagues (2016) compare responses of individuals from 157 countries to the standard Cantril Ladder life satisfaction question ( $0=$ low, to $10=$ high, see Cantril, 1963), and determine the mean and standard deviation of life satisfaction for each country. The standard deviation of SWB is the most common measure of SWB inequality in the literature (Bolle et al., 2009; Goff et al., 2016; Kalmijn \& Veenhoven, 2005; Ott, 2005)

Helliwell and colleagues (2016) provide useful contextual information regarding the state of world happiness and happiness inequality. Bhutan (a country which uses Gross National Happiness as its primary indicator of progress) had the lowest standard deviation of life satisfaction globally (1.294), while the country with the highest standard deviation was South Sudan with more than double that of Bhutan (3.044). While ranking $8^{\text {th }}$ globally for mean life satisfaction (at 7.334), New Zealand was ranked $18^{\text {th }}$ globally for the standard deviation of life satisfaction (1.649), behind a mixture of both developed and developing nations.

Helliwell and colleagues (2016) also demonstrate that SWB inequality has grown overall from the period of measurement in 2005-2011 to 2012-2015. Bearing this in mind, perhaps their most important finding is evidence of an association between higher wellbeing inequality and lower mean wellbeing in nations. Like Helliwell and colleagues (2016), most studies tend to look at the distribution of SWB at the country level (Abdallah \& Quick, 2016; Becchetti et al., 2013; Dutta \& Foster, 2013; Goff et al., 2016; Ott, 2005; 2011; Stevenson \& Wolfers, 2008; Veenhoven, 2000; 2005).

However, only a few researchers have explicitly examined the relationship between wellbeing and wellbeing inequality. For example, Delhey (2004) used data on life satisfaction in 28 European countries and found a negative relationship between the 
16|P a g e

standard deviation and mean levels of life satisfaction in nations. Fahey and Smyth (2004) do a similar analysis of happiness data from 33 European nations, and find that happiness has a close negative relationship with happiness inequality. Likewise, Bolle and colleagues (2009) use data for 71 countries to look directly at the link between the standard deviation and average level of happiness in countries. They conclude that greater equality in happiness is one of the main drivers of increasing happiness levels. Ott (2005) uses data on 78 countries from the World Values Survey and finds a consistent negative relationship between life satisfaction and life satisfaction inequality.

Goff and colleagues (2016) conduct the most comprehensive international analysis using the European Social Survey, the World Values Survey, and the Gallup World Poll to demonstrate that life satisfaction is negatively related to life satisfaction inequality in each survey. They also analyse the Gallup-Healthways Well-Being Index, which collects life satisfaction data from individuals in the United States. Controlling for age, sex, marital status, and education, Goff and colleagues (2016) find a strong negative relationship between life satisfaction and life satisfaction inequality by state. ${ }^{2}$

In summary, country-level research indicates that average wellbeing declines as wellbeing inequality increases. Research so far has primarily explained these results using country-level characteristics and indicators (Ott, 2005; 2011; Ovaska \& Takashima, 2010; Veenhoven, 2000; 2005). For instance, Ott (2005; 2011) has repeatedly demonstrated that good quality governance and a number of other country characteristics offer some explanation for the relationship between mean and standard deviation SWB. Those studies examining SWB inequality outside of its relationship with average wellbeing generally also offer institutional explanations for varying SWB inequality (Becchetti et al., 2013).

However, explaining SWB inequality solely using country-level characteristics begs the question: what if wellbeing varies at the sub-national level? If the relationship between wellbeing and wellbeing inequality differs between regions in the same country where institutional effects are constant, then there must be other mechanisms underlying the relationship. The following section addresses the literature discussing SWB and SWB inequality at the sub-national spatial scale.

\footnotetext{
${ }^{2}$ A graph and results from each of Delhey (2004), Ott (2005), Fahey and Smyth (2004), and Goff and colleagues (2016) will be reproduced in Figure 3.1, alongside discussion of the regression models used in this thesis.
} 


\section{5: Sub-National Wellbeing Inequality}

Hardly any research measures SWB inequality at a scale more specific than the national level. This section discusses research which has analysed SWB inequality in sub-national spatial groupings to date.

In one of the few articles examining sub-national wellbeing inequality at a smaller scale, Okulicz-Kozaryn (2011) compares mean life satisfaction and life satisfaction inequality in a number of provinces of Western European countries. While pointing to their marked differences, Okulicz-Kozaryn does not examine a possible relationship between mean SWB and SWB inequality.

The only study to explicitly examine sub-national sensitivity of wellbeing to wellbeing inequality is Tesfazghi and colleagues (2010). The authors use data on subjective quality of life (measured on a 6-point Likert scale) collected in 11 small administrative units in the Kirkos sub-city of Addis Ababa in Ethiopia. They note that as the variance in quality of life in these administrative units increases, mean quality of life decreases. However, as it is not the focus of their discussion, Tesfazghi and colleagues offer no explanation for this relationship (2010). Nonetheless, their findings indicate that country scale studies can hide variability in SWB at smaller scales, indicating a need for deeper exploration of possible sub-national relationships between SWB and SWB inequality.

The variation in SWB within countries (such as across regions) likely has some bearing on average levels of wellbeing in those places, but this relationship is currently unexplored. The relationship at a sub-national level could not be explained solely by the country-level institutional effects currently offered in the literature. So why should humans be sensitive to the variation in SWB within regions? I have covered some of the theoretical explanations in section 2.3, and it is these explanations which my thesis will apply to the SWB and SWB inequality relationship in the following research hypotheses.

\section{6: Research Hypotheses}

Research has consistently found that both mean and individual SWB decrease with rising SWB inequality (Bolle et al., 2009; Delhey, 2004; Fahey \& Smyth, 2004; Goff et al., 2016; Helliwell et al., 2016; Ott, 2005). On the basis of the literature I advance the following four hypotheses, which I test using sub-national spatial groupings within New Zealand. 
1. The Primary Hypothesis: Wellbeing is negatively associated with wellbeing inequality at sub-national scales.

2. The Parochialism Hypothesis: Wellbeing is more sensitive to wellbeing inequality at smaller, more localised scales.

3. The Fairness Hypothesis: Those who perceive society to be fairer are less sensitive to wellbeing inequality.

4. The Altruism Hypothesis: Individuals who hold altruistic values are more sensitive to wellbeing inequality.

\section{7: Summary}

Unlike the literature on income inequality and wellbeing, the literature on the relationship between wellbeing inequality and wellbeing has demonstrated a consistent negative relationship at the international level. To date, this relationship has been explained largely by country-level institutional effects.

In contrast, there is little research examining the wellbeing and wellbeing inequality relationship at the sub-national level. What does exist indicates a negative relationship is likely. The country context is constant at the sub-national level, meaning that the institutional explanations used at the international scale are unlikely to drive the relationship.

Existing research on the sensitivity of SWB to income inequality, and inequality aversion more broadly, offers some clues as to what might drive the relationship between wellbeing and wellbeing inequality at the sub-national level. The egalitarian nature of altruistic individuals means they should be more sensitive to wellbeing inequality than their less altruistic counterparts. Those who perceive society to treat people fairly should be less sensitive to inequalities in wellbeing than those who are more sceptical. Finally, parochialism indicates that sensitivity to the dispersion of wellbeing should be greater at increasingly localised scales.

The following chapter addresses the relationship between wellbeing and wellbeing inequality within countries at a conceptual level, before discussing the methods and surveys used in this research in the analysis chapters. 


\section{Chapter 3: Conceptual and Methods Chapter}

If a sub-national relationship between wellbeing and wellbeing inequality exists, it has implications for the way we approach both research and policy on wellbeing. Previous literature informs us that the sub-national relationship between wellbeing and wellbeing inequality is likely to be negative (Fahey \& Smyth, 2004; Goff et al., 2016; Ott, 2005; Tesfazghi et al., 2010). However, we still know little about how to explain this sub-national relationship, if it does exist. This chapter focuses on the key terms of the wellbeing and wellbeing inequality relationship.

The first portion of this chapter defines wellbeing and its measures. The second section discusses the expected relationship between wellbeing and wellbeing inequality, with examples from previous research. The third section of the chapter contains my discussion of the possibility that the form of the SWB and SWB inequality relationship may differ according to geographic scale.

The fourth section discusses measuring wellbeing inequality. In the fifth section, I detail the statistical model used in this thesis. I then go on to introduce individual attributes in the sixth section by discussing how the intercept and slope of the relationship might differ between people with different characteristics. The final section introduces the three surveys prior to their analysis in chapters 4 to 6 .

\section{1: Measuring Wellbeing}

What is subjective wellbeing (SWB)? While a succinct definition was provided in the introduction chapter, this section goes into greater depth regarding SWB as it is used in the literature. The key distinction from objective measures of wellbeing such as income is the emphasis on 'subjective': the person's own evaluation of their wellbeing.

SWB has been measured in a multitude of ways. The primary distinction that economists make is between affective happiness (the presence of positive or negative emotions) and evaluations of how well life as a whole is going (Helliwell, Layard \& Sachs, 2013). Life evaluation measures tend to be more stable over time, meaning they are more useful when trying to determine the impact of inequality, and are the most commonly used in the SWB inequality literature (Fahey \& Smyth, 2004; Helliwell et al., 2016; Ott, 2005). As a result, my research focuses exclusively on life evaluation measures of SWB. 
Among life evaluation measures, the most commonly used in research is life satisfaction. In Chapter 1, I referred to the New Zealand Quality of Life Survey life satisfaction question as fairly typical of those used in the literature ("Taking everything into account, how satisfied or dissatisfied are you with your life in general these days?"). Respondents answered on a 5-point Likert scale ranging from 'very dissatisfied' (1) to 'very satisfied' (5).

Other measures are widely used as well, such as happiness with life as a whole, or overall quality of life (Helliwell et al., 2013). While these measures may all seem similar at face value, it is important to recognise that they capture distinct aspects of life, as individuals can respond differently to each of them within the same survey (Morrison, 2007). In spite of the differences between various SWB measures, terms such as 'happiness', 'life satisfaction' and 'subjective wellbeing' are often used interchangeably. Throughout this thesis I will refer to the specific measure where applicable, and will use the terms 'subjective wellbeing' or simply 'wellbeing' when discussing the concepts more broadly.

Most SWB is measured on Likert scales like that in the Quality of Life Survey. These scales can range from a 3-point scale (Dutta \& Foster, 2013) up to 11-point scales (Cantril, 1963). When using scales of most sizes, SWB can be treated as either cardinal or ordinal (Ferrer-i-Carbonell \& Frijters, 2004). For instance, Dutta and Foster (2013) use data from a life evaluation question about how happy someone is in their day-today life that offers a choice of 'not too happy', 'pretty happy', or 'very happy'. In the aforementioned instance, we might consider the data ordinal, as there are only three points and we don't know the numerical 'distance' between each value (i.e. whether the difference between 'not too happy' and 'pretty happy' is the same as that between 'pretty happy' and 'very happy').

Ferrer-i-Carbonell and Frijters (2004) test the difference between assuming cardinality or ordinality when using happiness measures, and find it makes little difference to the results which one is assumed. The measures I use in this thesis range from 5-point to 11-point scales, at which cardinality assumptions are acceptable. As such, in this thesis I follow the majority of the literature in treating SWB as cardinal.

\section{2: What is the Relationship Between Wellbeing and Wellbeing Inequality?}

The four empirical studies to date demonstrate a strong negative relationship between mean wellbeing and wellbeing inequality: clockwise are Delhey (2004), Fahey \& Smyth (2004), Ott (2005) and Goff and colleagues (2016: reconstructed from their 
original data set and based mainly on OECD countries). Figure 3.1 displays a graph from each of these papers as an example. Note that they all plot mean life satisfaction on the $y$ axis and life satisfaction inequality on the $x$ axis, with the exception of Ott (2005) who reverses the axes.

Figure 3.1: Four examples of inter-country negative relationships between wellbeing and wellbeing inequality
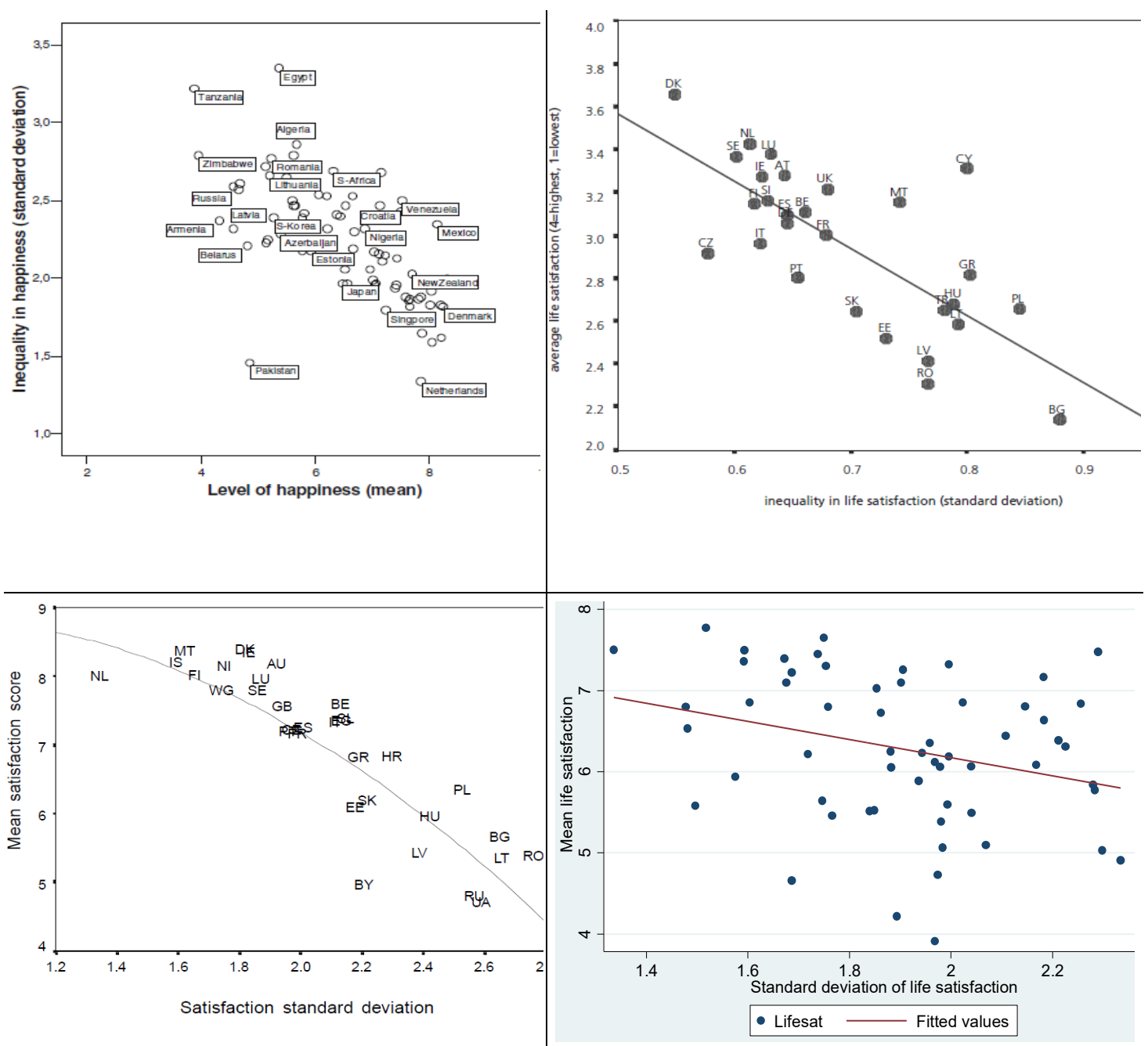

Figure 3.1: Sources, clockwise: Ott (2005), Delhey (2004), Goff and colleagues (2016), Fahey \& Smyth (2004)

I will now discuss the magnitude of the relationships in Figure 3.1 in greater detail. Ott (2005) investigates the relationship between mean life satisfaction and life satisfaction inequality in 78 countries using World Values Survey data from 1999-2001. In Figure 3.1, Ott finds a negative correlation between inequality in life satisfaction (10point Likert scale) and the level of life satisfaction: greater inequality goes with lower means (Pearson $r=-.65$ ). 
Delhey (2004) draws on the Candidate Countries Eurobarometer (for acceding and candidate European Union countries) and the standard Eurobarometer to compare mean life satisfaction (4-point scale) and life satisfaction inequality across 28 European nations. As demonstrated in Figure 3.1, Delhey finds a negative relationship, but does not report the magnitude or significance of this regression (2004).

Fahey and Smyth (2004) conduct a similar analysis, producing the graph in Figure 3.1 to demonstrate a negative relationship between mean life satisfaction (10point scale) and life satisfaction inequality for 33 countries from the 1999-2000 European Values Survey. They provide an $\mathrm{R}^{2}$ of $77 \%$ for their regression, indicating inequality in life satisfaction accounts for a great deal of the variation in mean life satisfaction (Fahey \& Smyth, 2004).

Goff and colleagues (2016) use Cantril Ladder 11-point life satisfaction measures drawing on a variety of data sources. Their data was made publically available as an online resource accompanying the World Happiness Report 2016. I draw on the 58 countries with a GDP per capital (ppp) of US\$13,359 and above to construct the graph in Figure 3.1 and estimate a negative relationship between the variables $(b=$ $1.115, p<0.05)$.

Based on this literature, I expect mean wellbeing in New Zealand regions to fall as regional wellbeing inequality rises. In each inter-country case the relationship between the countries' average wellbeing and wellbeing inequality is linear ${ }^{3}$.

\section{3: Geographic Scale}

Largely unaddressed in the inter-country studies is the possible influence of geographic scale and the relative size of the spatial groups (e.g. the city or region). We do not know whether to expect small and large settlements to bear the same relationship between average wellbeing and the observed dispersion of wellbeing within them. Tobler's First Law of Geography states that everything is related to everything else, but that near things are more related than distant things (Tobler, 1970). Applying Tobler's First Law alongside the theory of parochialism, we would expect that people are more sensitive to the SWB of those who are close to them compared to those who are distributed throughout the wider region or nation (see Figure 3.2).

\footnotetext{
${ }^{3}$ There is an a priori case for non-linearity as well, in which average wellbeing falls at an increasing rate with rising dispersion in wellbeing. However, the possibility of a non-linear relationship has no consistent empirical support in the literature.
} 
Figure 3.2: Sensitivity of average wellbeing to wellbeing inequality at different scales

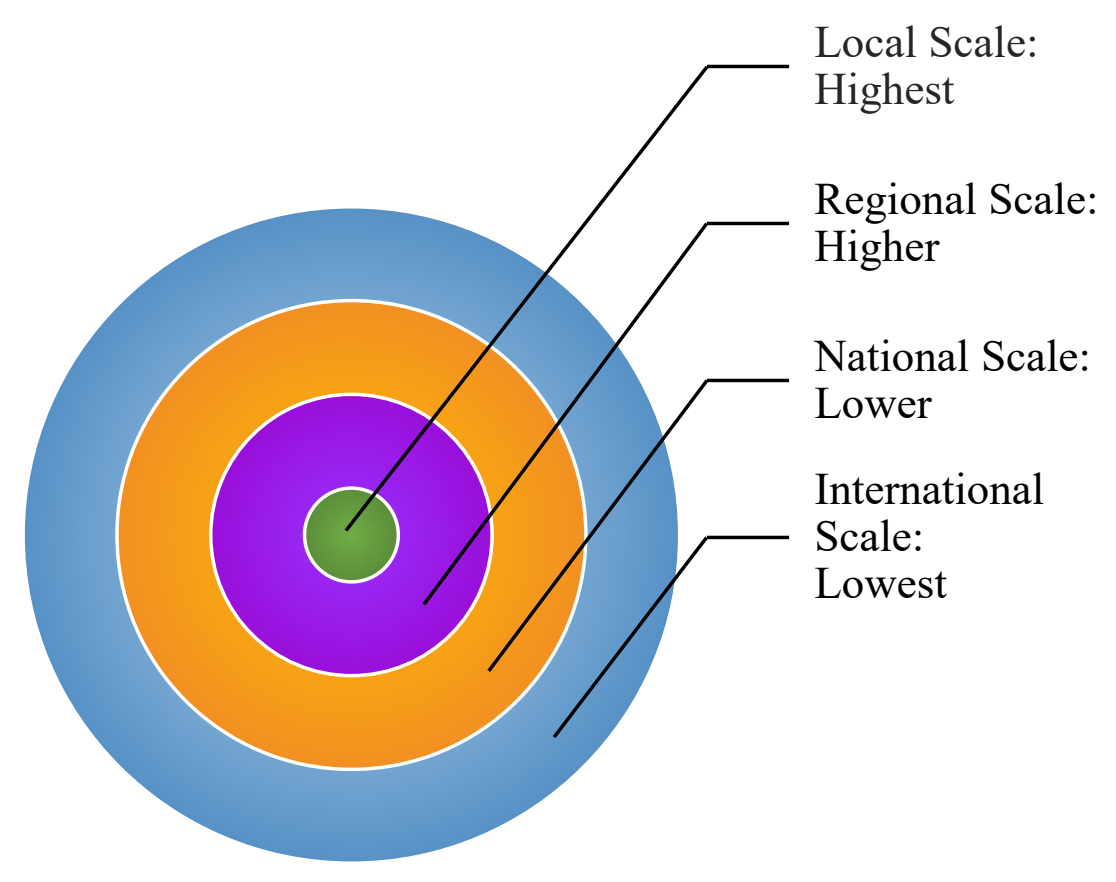

According to The Parochialism Hypothesis, the size or scale of the spatial group can be expected to influence the strength of the relationship between wellbeing and wellbeing inequality. Figure 3.3 shows the predicted relationship between SWB and SWB inequality at different geographic scales, if the interaction between the two is indeed linear. The solid blue line represents the relationship we might expect to see at the national scale, where people are expected to be less sensitive to inequalities in SWB. The dashed blue line represents the relationship that might be found at the regional level, where parochialism indicates we are theoretically more sensitive to SWB inequality. Finally, the dotted blue line represents the local area, where we would expect people to be the most sensitive to inequalities in the wellbeing of those around them. 
$\mathbf{2 4} \mid \mathrm{P}$ a g e

\section{Figure 3.3: The theoretical sensitivity of wellbeing to wellbeing inequality at different sub-national scales}

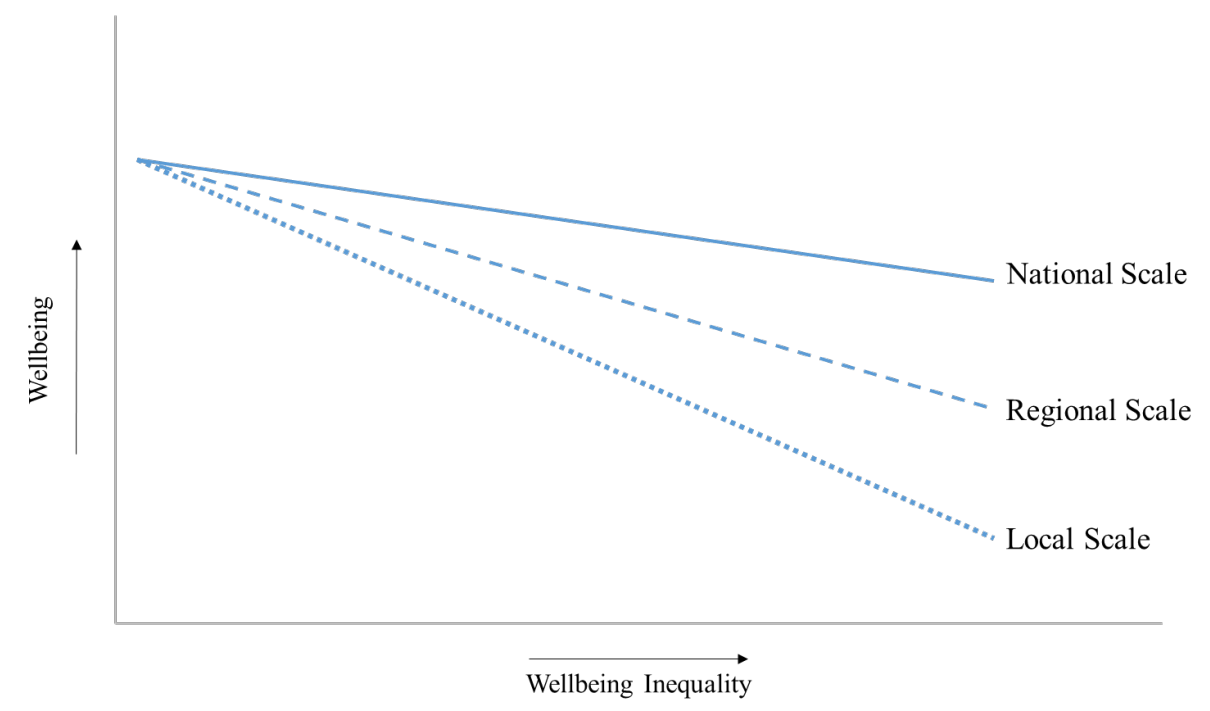

\section{4: Measuring Inequality}

In their much-cited report on measuring social progress, Stiglitz, Sen and Fitoussi (2009) highlight the following in a section on inequality:

"While established methodologies and data sources can be used to measure inequalities in the distribution of economic resources in a fairly reliable way, the situation is much less satisfactory with respect to the non-monetary dimensions of quality of life." (p. 50).

The literature on wellbeing inequality follows the income inequality literature in many respects. However, even though wellbeing can be treated as cardinal (Ferreri-Carbonell \& Frijters, 2004), SWB measures themselves are still ordinal, unlike income. SWB is measured on a bounded scale and is therefore subject to a 'ceiling effect' whereby it is unable to increase beyond a certain point (Goff et al., 2016).

When measuring standard deviation based on a bounded scale, a potential problem arises if the distribution is negatively skewed. If a large number of respondents' life satisfaction ratings appear near the top of the scale, the presence of the upper bound artificially reduces the standard deviation value, leading to a negative mechanical or structural relationship between wellbeing and wellbeing inequality. For a more comprehensive explanation of the issues associated with a bounded scale, see Quick (2015). 
In practice, the standard deviation will only be distorted by structural dependency when the mean is very close to the upper bound. When measuring SWB using 11-point or 10-point Likert scales, such extreme means very rarely occur (Kalmijn $\&$ Veenhoven, 2005). In practice therefore few means encountered in the literature are likely to be affected by the 'mechanical' effect. This 'problem' and the suitability of standard deviation as a measure of SWB inequality has been the subject of thorough debate (Delhey \& Kohler, 2011; Dutta \& Foster, 2013; Kalmijn, 2012; Veenhoven, 2012), and the majority of researchers now agree that the standard deviation measure of inequality is suitable (Berg \& Veenhoven, 2010; Bolle et al., 2009; Kalmijn \& Arends, 2010; Kalmijn \& Veenhoven, 2005; Ott, 2005; Ovaska \& Takashima, 2010).

Two recent articles address structural dependency and assumptions of cardinality in SWB inequality research in greater depth. In a comprehensive evaluation of structural dependency in the relationship between wellbeing and wellbeing inequality, Goff and colleagues (2016) conclude that structural dependency is hardly an issue in practice. Clark, Fléche and Senik (2016) compare an ordinal measure of SWB inequality with the standard deviation of SWB, and likewise find that the choice had little bearing on the overall results. So, the standard deviation remains the most acceptable and convenient measure of SWB inequality. As such, I employ it in the models below.

\section{5: Modelling the Wellbeing and Wellbeing Inequality Relationship}

In modelling the relationship between the wellbeing and wellbeing inequality of a place, I make a distinction between correlating measures of the group and of individuals. In other words, I distinguish between running ecological regressions and individual regressions.

\section{The Ecological Relationship}

The only groups used in relating average wellbeing to wellbeing inequality in the literature so far are spatial groupings of countries. The regressions are therefore appropriately termed ecological regressions (Robinson, 1950). In the ecological or group model the dependent variable is the mean of life satisfaction in an area, $\bar{S}_{g}(1)$.

(1) $\bar{S}_{g}=1 / n \sum_{i=1}^{n} S_{i}$ 
Where $S_{i}$ is the life satisfaction reported by the $i^{\text {th }}$ person, $i=1, \ldots, \mathrm{N}$. The measure of wellbeing inequality is the standard deviation of life satisfaction within the group, $D_{g}$, equation (2).

(2) $D_{g}=\sqrt{ } 1 / n \sum_{i=1}^{n}\left(S_{i}-\overline{\mathrm{S}}_{g}\right)^{2}$

The ecological model relates the average wellbeing of the geographic group $\bar{S}_{g}$ (1) to the standard deviation of wellbeing within the group (2).

(3) $\quad \bar{S}_{g}=f\left(D_{g}\right)$

In its expanded form we can see that how $S_{i}$ appears on both sides of equation (4).

$$
\frac{1}{n} \sum_{i=1}^{n} S_{i} \quad=\quad f \sqrt{ } 1 / n \sum_{i=1}^{n}\left(S_{i}-\bar{S}_{g}\right)^{2}
$$

When the following ecological model has been applied as a full regression, the estimated coefficient has been found to be negative in all cases. In other words, the average wellbeing of the group decreases with every unit increase in its standard deviation wellbeing, equation 5.

(5) $\bar{S}_{g}=a_{o}+\beta D_{g}+\varepsilon_{g}$

While the ecological model is a convenient and simple way of analysing the relationship between mean and standard deviation of life satisfaction, Robinson (1950) emphasises that ecological models may not accurately depict the relationship as it applies at the individual level. The following section introduces the individual model to be used alongside the ecological model in this research.

\section{$\underline{\text { The Individual Model }}$}

In the applications below, the individual model (6) differs from the ecological model in only one respect: the individual replaces the group as the unit of analysis, and therefore the $\mathrm{N}$ becomes much larger $(i=1, \ldots, \mathrm{N}>g=1, \ldots, \mathrm{n})$. Under this model the wellbeing of individual $i$ (in group $g$ ) is regressed on the standard deviation of the group they are a member of. The primary reason for applying this model is to allow the 
attributes of individuals to also serve as arguments, $X_{i g}$ (e.g. we might want to know the influence being unemployed has on a person's SWB).

$$
S_{i g}=a_{o}+\beta D_{g}+X_{i g}+\varepsilon_{i g}
$$

According to equation 6, the life satisfaction of the individual is expected to be (negatively) affected by the degree of inequality in life satisfaction across the spatial group that they are a member of. In addition, the equation will predict the marginal effects of the attributes included in X (e.g. age, sex, income) and these in turn will moderate the effect of inequality.

A variant of equation 6 is applied when I want to test whether the effect of the inequality within the group on an individual's wellbeing is sensitive to the beliefs people have. For example, in chapters 5 and 6 I ask whether an individual believing society is fair ( $F=1$ vs 0$)$ affects their sensitivity to local wellbeing inequality. This test is undertaken by adding the fairness variable and an interaction term as in equation 7 , generating parameters $\zeta$ and $\lambda$ respectively. Extensive use is made of this model by Goff (2005) among others.

$$
S_{i g}=a_{o}+\beta D_{g}+\zeta F_{i}+\lambda(F . D)_{i g}+X_{i g}+\varepsilon_{i g}
$$

In the applications of the individual model I also recognise the presence of spatial clustering and its potential effect on the iid assumptions of the model. I therefore apply the standard adjustment for clustered observations (using Stata 14). All results in this thesis are adjusted for clustering according to the geographic variables in the different surveys.

In chapters 5 and 6 I test explicitly for the effect that perceiving a fair society or having altruistic tendencies has an individual's sensitivity to SWB inequality. In addition to reporting the estimates I also present graphs of the predicted SWB (the marginal) along with the confidence bands of those estimates (see Figure 5.4 for an example). These figures allows me to convey visually the relative effect of believing society is fair or of proxies for altruism.

There is a third, more advanced type of model applied in the literature which combines (as two levels) the ecological and the individual. Referred to as either the random coefficients or multi-level model, this model has a number of advantages over the standard cluster adjusted Ordinal Least Squares (OLS) regression model I use. In 
$\mathbf{2 8} \mid \mathrm{P}$ a g e

particular it allows the variance in wellbeing to be partitioned across level 1 (the individuals), and level 2 (the place). Most usefully it allows an explicit test for interactions between characteristics of the place and attributes of the individual. The model can also be extended to successively larger places nested within each other (the hierarchical model). The application of this more sophisticated model would go beyond the scope of a master's thesis. However, multi-level modelling is increasingly applied to SWB, and warrants using in future research (Aslam \& Corrado, 2011; Ballas \& Tranmer, 2012; Pittau, Zelli \& Gelman, 2010).

The Distribution of Wellbeing and Wellbeing Inequality

For the individual regression, it is useful to visualise the distribution of individual life satisfaction and the life satisfaction inequality of their groups. Figures $3.4,3.5$, and 3.6 display histograms of life satisfaction for each of the surveys used in this thesis. Figure 3.7 does the same for standard deviation life satisfaction in the quality of life survey.

Figure 3.4: Histogram of life satisfaction in the Quality of Life Survey for 2010 and 2012

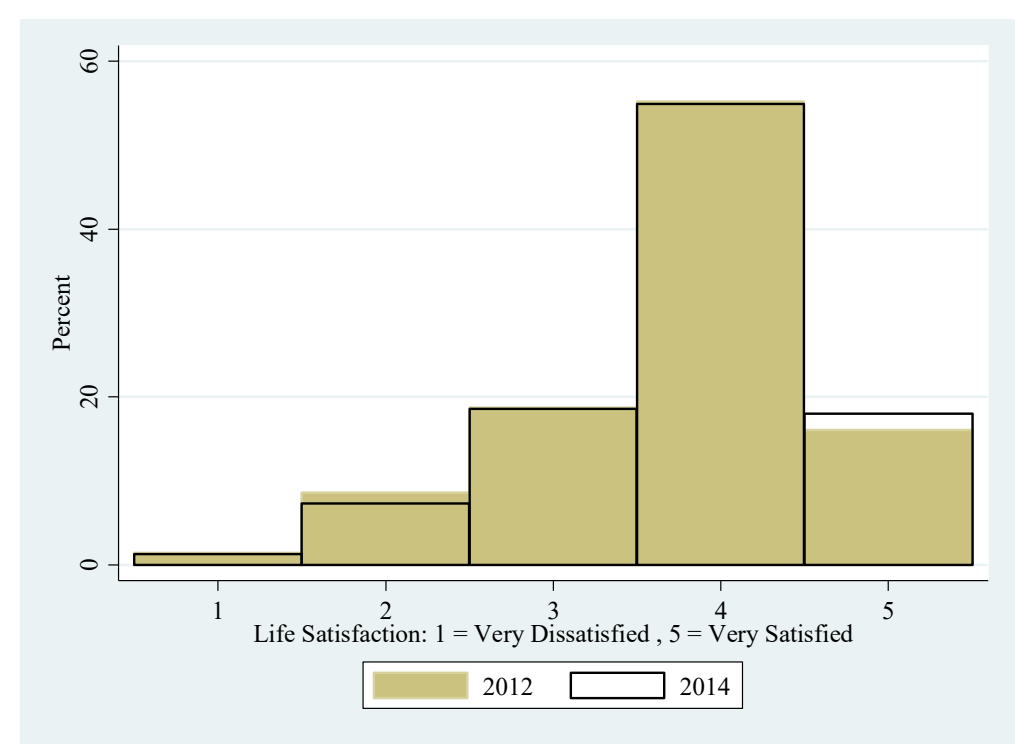

Figure 3.4: Quality of Life Survey, 2012 (khaki) and 2014 (black outline). Mean life satisfaction is 3.759 in 2012, and 3.812 in 2014. 
Figure 3.5: Histogram of life satisfaction in the New Zealand General Social Survey for 2008, 2010, and 2012

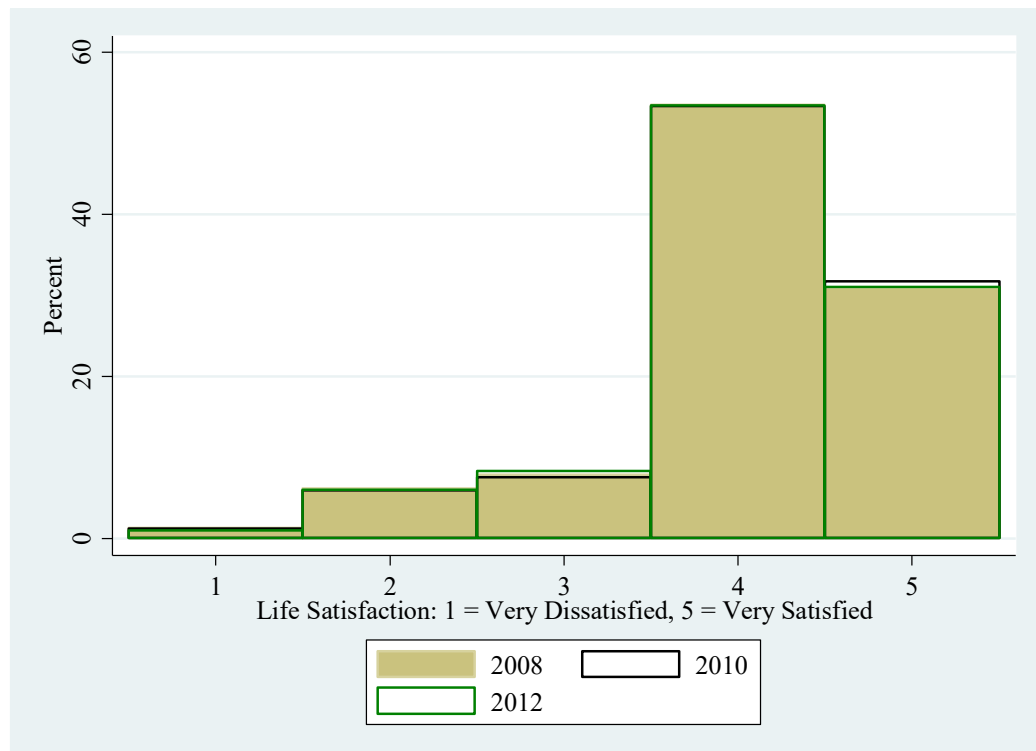

Figure 3.5: New Zealand General Social Survey, 2008 (khaki), 2010 (black outline), and 2012 (green outline). Mean life satisfaction is 4.067 in 2008, 4.086 in 2010, and 4.075 in 2012.

Figure 3.6: Histogram of life satisfaction in Te Kupenga

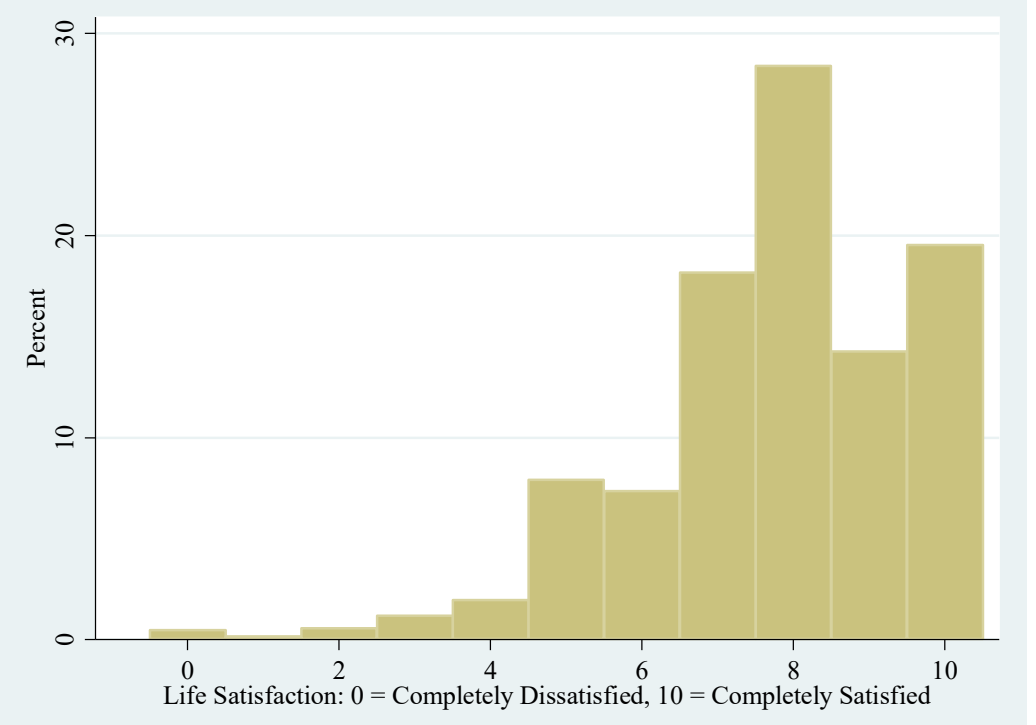

Figure 3.6: Te Kupenga, 2013. Mean life satisfaction is 7.744. 
30|P a g e

Figure 3.7: Histogram of the standard deviation life satisfaction of wards in the quality of life survey

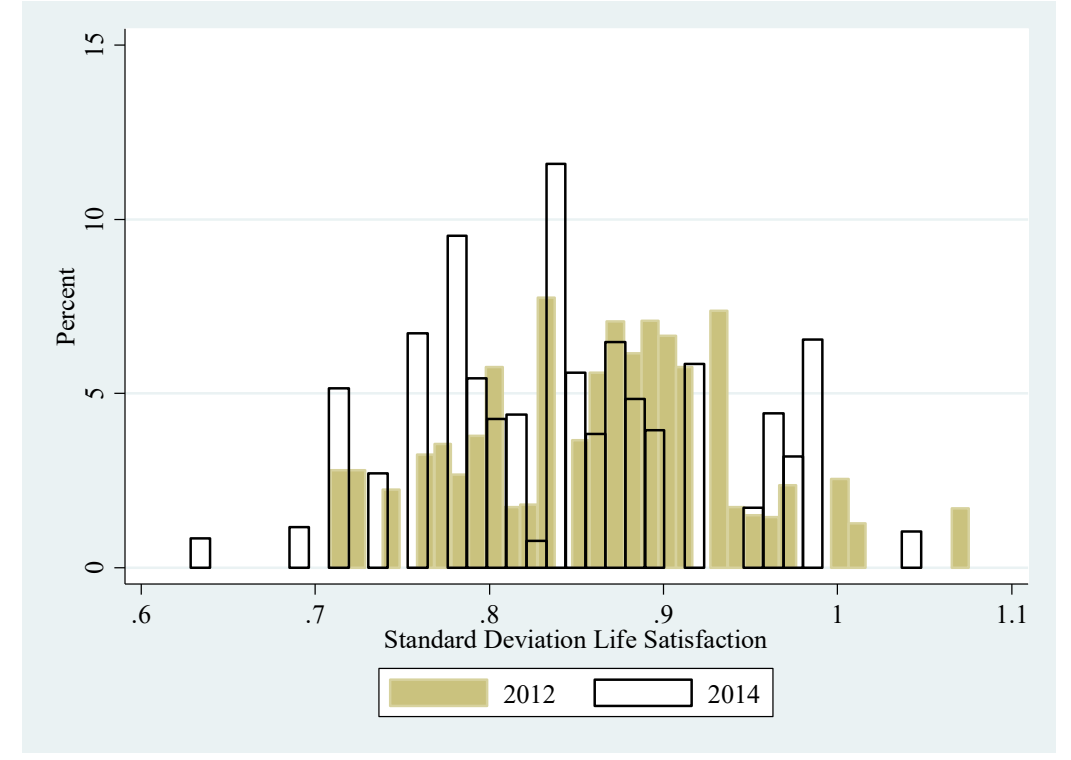

Figure 3.7: QoLS, 2012 (khaki), 2014 (black outline). Mean standard deviation life satisfaction by ward is 0.863 in 2012 and 0.846 in 2014 .

A few things are evident from Figures 3.4 to 3.7. First and foremost, life satisfaction is not normally distributed in any of the three surveys, exhibiting the negative skew I expect. It also seems Te Kupenga has a higher cluster of respondents with high wellbeing than the other surveys, although this may simply be due to the different scales. I test this by temporarily collapsing the 11-point life satisfaction scale into a 5-point scale (combining ' 0 ' in with ' 1 ' and ' 2 ') and comparing the mean with the other surveys. With a mean life satisfaction of 4.085, it appears as though the distribution of life satisfaction in Te Kupenga is roughly similar to that of the other surveys when viewed on the same scale (see Figure captions).

Figure 3.7 demonstrates the distribution of standard deviation life satisfaction values throughout the Quality of Life Survey (the other surveys were not used owing to the small number of spatial categories - to be discussed later). The distribution appears roughly bi-modal in 2012, and possibly even tri-modal in 2014, indicating some changes in clustering over time. Those with extremely high values on either end only have a few observations within them, indicating they may be outliers. The spread of standard deviation life satisfaction values will be discussed in greater depth within the analyses. 
So far I have mentioned the likelihood that individual attributes impact on the relationship between wellbeing and wellbeing inequality. However, I have not yet explored the manner in which those personal attributes might have an impact. The following section briefly discusses the way personal attributes might affect the intercept of the relationship as well as the slope.

\section{6: Introducing Individual Attributes}

Individual attributes of respondents to wellbeing surveys are important to consider for two reasons. First, they can be associated with differences in an individual's levels of wellbeing (the intercept $\alpha$ in the models above), and second, differences in attributes may alter the slope of the relationship between wellbeing and wellbeing inequality (the parameter $\beta$ ).

All SWB research includes a few controls for attributes which are known to influence an individual's wellbeing. These attributes include factors such as age (or age group), sex, race, social connectedness (or another social capital indicator), and income. However, the variables that moderate the effect of SWB inequality in an area are less well documented.

\section{Demographic Characteristics}

The estimated effect of sex differences vary considerably, with females being happier than males in some places, and the opposite holding true in others (Dolan, Peasgood and White, 2008). Once controls are introduced, sex differences are often either substantively or statistically insignificant (Dolan et al., 2008; Fortin, Helliwell \& Wang, 2015). Sex may also mediate the relationship between wellbeing and wellbeing inequality. For instance, research has indicated females are generally more altruistic (Rand et al., 2016; Simmons \& Emanuele, 2007) so we might expect them to be more sensitive to inequality than males.

One of the most consistent findings in the SWB literature concerns the identification of a U-shaped relationship between age and SWB. In a given population, younger and older people tend to have higher wellbeing than middle-aged people (Dolan et al., 2008; Layard, Clark \& Senik, 2012). However, the U-shaped trend is primarily found in more developed countries, with many developing countries exhibiting a negative relationship between age and wellbeing (Fortin et al., 2015). Furthermore, evidence indicates this U-shaped trend may also vary from place to place even in developed nations (Fortin et al., 2015). Regarding SWB inequality in particular, 
Becchetti and colleagues (2013) found an inverse U-shaped relationship with age groups, with members of the middle-age group (45-54) having the highest inequality in life satisfaction.

The age of an individual could also alter the slope of the wellbeing and wellbeing inequality relationship. There is evidence that the elderly are more sensitive to income inequality (Morrison, 2015b), indicating they may also be more sensitive to SWB inequality. Alternatively, there is evidence that happiness inequality is greater among the middle-aged groups (Becchetti et al., 2013), so it may be that the middleaged are actually the most sensitive to wellbeing inequality.

Findings on race generally conclude that a difference in SWB does exist, with people of colour generally reporting lower wellbeing than whites in more developed countries such as the United States (Dolan et al., 2008). Racial differences in SWB have received attention in New Zealand, given the traditional disparities in outcomes faced by New Zealand's indigenous Māori (Sibley, Harré, Hoverd \& Houkamau, 2011). Sibley and colleagues (2011) found the gap between the SWB of Māori and New Zealand Europeans has widened significantly over time, with Māori becoming less satisfied and New Zealand Europeans staying roughly the same. As such, racial differences are necessary to control for in any SWB research conducted in New Zealand.

On a related note, cultural background can significantly alter the way an individual reports their SWB. For instance, Latin Americans and some Middle Eastern cultures consistently report higher SWB than would be predicted based on their objective characteristics (Brulé \& Veenhoven, 2017; Dolan et al., 2008). The same may also apply for cultures in New Zealand (e.g. Māori).

Research has consistently indicated unemployment has a large negative impact on an individual's SWB, even after controlling for financial satisfaction (Alesina et al., 2004; Dolan et al., 2008; Fahey \& Smyth, 2004; Layard et al., 2012). Research on wellbeing inequality in particular indicates that there is more inequality in wellbeing among the unemployed when compared to the employed, although the effect of retirement (i.e. willingly unemployed) is non-significant (Becchetti et al., 2013).

The relationship between income and SWB has been the subject of a vast amount of research. Easterlin (1974) first ignited debate when he identified what is now 
called the Easterlin Paradox: that at any point in time, richer people are happier than poorer people, but that as countries get richer, they do not get happier on average.

Countless articles have been written on the topic since, but income at the individual level has generally been shown to improve wellbeing up to a certain point, whereafter income has diminishing returns to wellbeing (Clark et al., 2008; Dolan et al., 2008; Layard et al., 2012). So, while obtaining greater income is beneficial, it becomes much less so the more that we earn.

With regard to SWB inequality specifically, Becchetti and colleagues (2013) find that SWB inequality is lower among the rich than it is among the poor, an effect that is significant and consistent over time. We might also expect sensitivity to wellbeing inequality to be lower among the rich, as they tend to perceive the greatest benefit from inequalities (Morrison, 2015b) and may be exposed to less of a distribution in wellbeing among their high-earning peers.

$\underline{\text { Health }}$

Physical and mental health correlate strongly with SWB. While physical illness and disabilities have a large negative impact on SWB (Dolan et al., 2008; Layard et al., 2012), Layard, Chisholm, Patel and Saxena (2013) found that mental illness is the single biggest determinant of human unhappiness in many countries. Given the close relationship between someone's health and their capacity to live the life they desire, it is perhaps unsurprising that health is one of the strongest determinants of SWB.

\section{$\underline{\text { Social Capital }}$}

Our social relationships and interactions with others are perhaps the most important factor in influencing our SWB. Social capital is a broad term encompassing our social relationships, trust in others, and our ability to rely on others in times of need (Sachs, 2015). Sachs (2015) describes a number of studies demonstrating that possessing high social capital is extremely beneficial to the wellbeing of both individuals and societies.

Breaking social capital down into more specific components, research consistently indicates that being married, socialising with family and friends, having a high level of trust in society, being pro-social, and interacting with the community all positively impact on SWB (Dolan et al., 2008; Layard et al., 2012; Sachs, 2015).

At present, I am not aware of any research considering the effect of social capital on the relationship between SWB and SWB inequality in a place. However, I would assume those who have high social capital are more sensitive to the inequality around 
them, as they are likely more invested in their community and more aware of the inequality within it. However, it is also possible that social capital plays a protective role and that more socially connected individuals are buffered from the negative impacts of SWB inequality.

It is worth noting that variables such as subjective health or social isolation likely have a high degree of endogeneity with SWB measures such as life satisfaction. As such, one should be careful when interpreting the results of regressions in which they are controlled for.

$\underline{\text { Values }}$

Researchers have begun to explore the effect that values might have on SWB (Morrison \& Weckroth, 2017), but values are traditionally not included as controls in SWB research, and so the literature using them as such will not be discussed here. Still, particular attention should be paid to the values of individuals when considering variations in sensitivity to wellbeing inequality, as these values are likely to have a very significant impact upon the relationship between individual wellbeing and wellbeing inequality. The possible impact of the following variables on this relationship will be examined in the analysis.

The first values I examine are an individual's perceptions of fairness in society. An individual's perceptions often hold greater sway over their SWB than the reality of their situation. If an individual perceives society to be unfair, it is likely they will be more sensitive to the distribution of SWB in their area. Alternatively, if an individual believes the distribution of wellbeing in their society is the result of fair processes, they are likely to be less sensitive to wellbeing inequality. Goff and colleagues (2016) find those who believe income inequality is too high are more sensitive to wellbeing inequality. However, perceptions of fairness specifically have not been tested in regards to the relationship between wellbeing and wellbeing inequality.

The second value I examine is altruism. I expect that those who are more altruistic are more sensitive to wellbeing inequality in their community, with their own wellbeing reducing more for each unit increase in inequality. Alternatively, if an individual is more avaricious by nature and less caring about others, we might expect them to be relatively insensitive to wellbeing inequality.

I have established that three broad aspects of individuals' lives will likely have an impact on the sensitivity of their wellbeing to the inequality in wellbeing around 
them. First, the impact of wellbeing inequality on individual wellbeing likely differs according to geographic scale. Second, an individual's values such as altruism or perceived societal fairness may alter their sensitivity to wellbeing inequality. Finally, control variables will account for variation in other aspects of people's lives, providing a more complete picture, but likely weakening the relationship between wellbeing and wellbeing inequality. Figure 3.8 presents a simple visual representation of these relationships.

Figure 3.8: A visual representation of the factors likely to influence sensitivity to wellbeing inequality

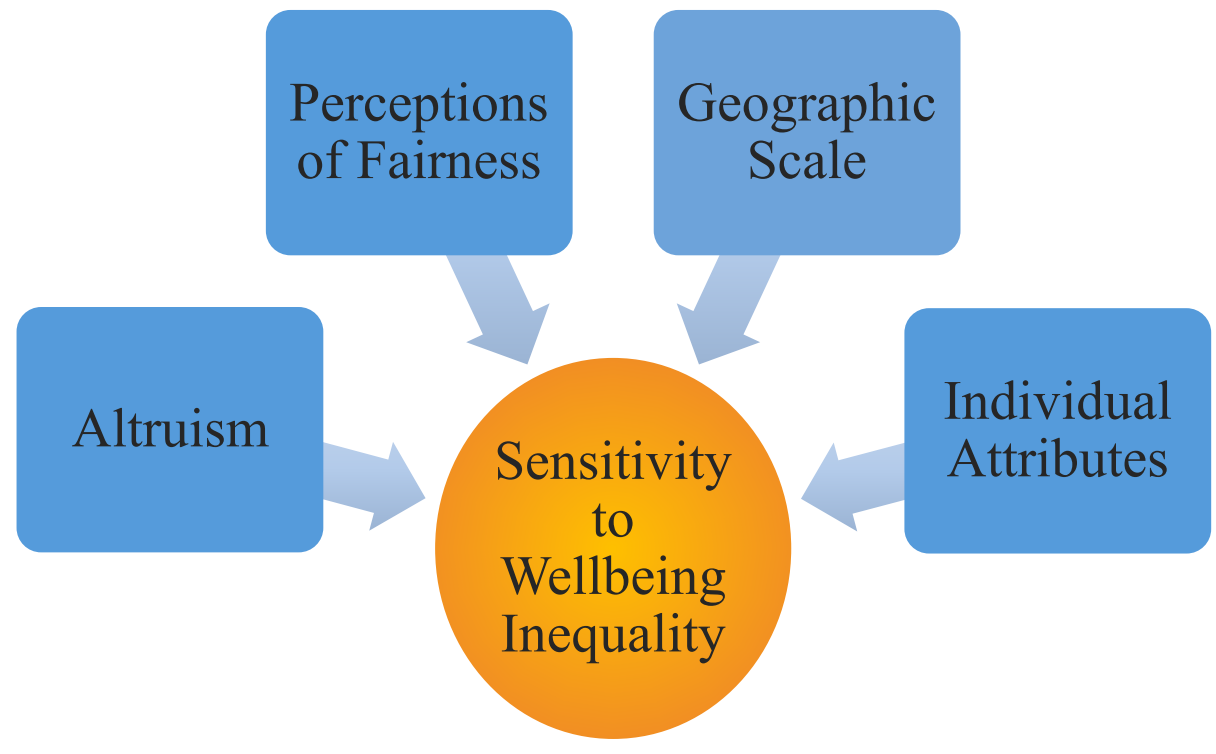

I have introduced the conceptual and statistical models that I will be using to examine the wellbeing and wellbeing inequality relationship, and have discussed the possible effects of individual attributes on this relationship. I now introduce the three surveys that the data are drawn from.

\section{7: An Introduction to the Survey Data}

Life satisfaction variables are available in the three surveys analysed in this thesis. In the New Zealand General Social Survey (NZGSS) and the Quality of Life Survey (QoLS) life satisfaction was measured using a 5-point Likert scale (see Figures 3.4 and 3.5). In Te Kupenga, however, life satisfaction was measured using an 11-point Likert scale (see Figure 3.6). ${ }^{4}$

${ }^{4}$ Detail on the specific wording of the SWB variables (and all other variables) is available in Appendix A. 
Given that I am going to be measuring variation within sub-populations of the three surveys, sample size is a particularly important consideration in my research. A total of 41,985 individuals responded to the life satisfaction questions across the three surveys, and are therefore available for analysis. Table 3.1 provides detail on the number of individuals who answered the life satisfaction question for each survey in each year. 5

Table 3.1: Number of responses to the life satisfaction question within each survey by year

\begin{tabular}{l|c|c|c|c|c|c|}
\multicolumn{2}{|c|}{$\mathbf{2 0 0 8}$} & $\mathbf{2 0 1 0}$ & $\mathbf{2 0 1 2}$ & $\mathbf{2 0 1 3}$ & $\mathbf{2 0 1 4}$ & Total \\
\hline \multirow{2}{*}{ NZGSS } & 8,710 & 8,543 & 8,455 & - & - & 25,708 \\
\cline { 2 - 7 } QoLS & - & - & 5,459 & - & 5,277 & 10,736 \\
\multirow{2}{*}{ Te Kupenga } & - & - & - & 5,541 & - & 5,541 \\
\cline { 2 - 8 } Total & 8,710 & 8,543 & 13,914 & 5,541 & 5,277 & 41,985 \\
\hline
\end{tabular}

While the SWB measures are the variables of interest for this thesis, the geographic variables are also essential. The geographic units in each survey will be used to generate the SWB inequality variables. When examining the individual scale relationship, each individual will be assigned a SWB inequality value representing the inequality in SWB within their area. When examining the relationship between mean SWB and SWB inequality, the geographic unit will be the basis for the creation of both the mean SWB and standard deviation SWB variables.

The QoLS contains a city variable with six cities, and a ward variable with 46 wards. The NZGSS contains a region variable with six regions, but also has an urban indicator with four different categories of urbanity. Te Kupenga likewise has a region variable with six regions as well as an urban indicator, but the urban indicator only has three categories. It is worth noting that some of the 'regions' in the NZGSS and in Te Kupenga span quite a large area with a relatively small sample size. More information on these geographic indicators will be provided in the analysis.

\footnotetext{
${ }^{5}$ For more information on the target populations, data collection processes and technical details, see Appendix A.
} 
Each survey also includes basic information on age, sex, ethnicity, employment, income, health, and social isolation. See Table 3.2 for basic summary statistics on these control variables as they appear in each survey. Only control variables present in all three surveys are presented in Table 3.2. Either mean or percentage values are displayed in the cells. The row containing the survey title describes how many groups or categories were available in total for each variable.

In each survey, individuals could choose multiple ethnicities, so only the percentage who chose New Zealand European are presented in Table 3.2. Health and isolation are measured on 5-point scales in all three surveys, with 1 being least healthy and least isolated, and 5 being healthiest and most isolated. The unemployed category includes only those actively looking for work (i.e. not retired individuals).

Table 3.2: Summary statistics on key control variables as they appear in each of the three surveys

\begin{tabular}{|c|c|c|c|c|c|c|c|}
\hline & $\begin{array}{c}\text { Mean } \\
\text { Age } \\
\text { Group }\end{array}$ & $\begin{array}{c}\text { Sex } \\
\text { (Male) }\end{array}$ & $\begin{array}{c}\text { New } \\
\text { Zealand } \\
\text { European }\end{array}$ & $\begin{array}{c}\text { Employment } \\
\text { Status } \\
\text { (Unemployed) }\end{array}$ & $\begin{array}{l}\text { Mean } \\
\text { Health }\end{array}$ & $\begin{array}{c}\text { Mean } \\
\text { Isolation }\end{array}$ & $\begin{array}{c}\text { Mean } \\
\text { Income } \\
\text { Group }\end{array}$ \\
\hline NZGSS & Fifteen & Binary & Multiple & Four & 5-point & 5-point & Thirteen \\
\hline 2008 & $45-49$ & $44.92 \%$ & $80.70 \%$ & $2.83 \%$ & 3.61 & 1.57 & $\begin{array}{l}\$ 25 \mathrm{k}- \\
\$ 30 \mathrm{k}\end{array}$ \\
\hline 2010 & $45-49$ & $44.15 \%$ & $80.48 \%$ & $3.70 \%$ & 3.60 & 1.54 & $\begin{array}{l}\$ 25 \mathrm{k}- \\
\$ 30 \mathrm{k}\end{array}$ \\
\hline 2012 & $45-49$ & $43.88 \%$ & $76.91 \%$ & $3.95 \%$ & 3.57 & 1.53 & $\begin{array}{l}\$ 25 \mathrm{k}- \\
\$ 30 \mathrm{k}\end{array}$ \\
\hline QoLS & Eleven & Binary & Multiple & Four & 5-point & 5-point & Ten \\
\hline 2012 & $45-49$ & $46.04 \%$ & $72.87 \%$ & $5.97 \%$ & 3.36 & 2.07 & $\begin{array}{l}\$ 40 \mathrm{k}- \\
\$ 50 \mathrm{k}\end{array}$ \\
\hline 2014 & $40-44$ & $45.96 \%$ & $69.83 \%$ & $6.59 \%$ & 3.42 & 2.04 & $\begin{array}{l}\$ 40 \mathrm{k}- \\
\$ 50 \mathrm{k}\end{array}$ \\
\hline $\begin{array}{l}\text { Te } \\
\text { Kupenga }\end{array}$ & Seven & Binary & Multiple & Three & 5-point & 5-point & Eight \\
\hline 2013 & $35-44$ & $44.14 \%$ & $54.44 \%$ & $6.17 \%$ & 3.48 & 1.66 & $\begin{array}{l}\$ 20 \mathrm{k}- \\
\$ 30 \mathrm{k}\end{array}$ \\
\hline
\end{tabular}


Gaining access to the QoLS involved emailing the primary council researcher involved in the Quality of Life Project to request permission to access the data for research purposes. Data was provided in the form of separate SPSS (Statistical Package for the Social Sciences) files. Data was then transferred to Excel for data cleaning purposes, after which it was imported into Stata for data analysis.

Access to both the NZGSS and Te Kupenga was gained by completing a confidentialised unit-record files (CURFs) access form and submitting it to the microdata access team at Statistics New Zealand. Access to the data and associated files was granted after signing the required terms of use forms. The NZGSS survey data was provided in SAS format and was converted to a Stata format for data cleaning and analysis.

It is important to note that much of the data originally collected in the NZGSS and Te Kupenga is not available to researchers outside of Statistics New Zealand, who are required to use the CURFs. CURFs are unit record data which have been altered to protect the confidentiality of individual respondents, whilst maintaining the integrity of the original data. A drawback of this modification from a research perspective is the removal of certain variables that may have been useful for analysis (such as more specific geographic identifiers), and the collapsing of variables into categories (e.g. age into age groups).

Whilst this data alteration does result in a slightly less rich dataset, it does assure Statistics New Zealand and associated researchers meet their ethical obligations to the anonymity and confidentiality of participants' responses. In spite of the confidentialised nature of the CURFs, the exploratory analysis which I present in this thesis will pave the way for future researchers to draw on more comprehensive datasets to explore the issues I present.

\section{8: Summary}

This chapter has provided conceptual and statistical models for the expected relationship between wellbeing and wellbeing inequality. I discussed the measurement of wellbeing inequality and concluded that the standard deviation of SWB is the most appropriate measure to use in this thesis.

I have presented models for both the ecological and individual regressions of the relationship between wellbeing and wellbeing inequality. I also discussed the 
possible impact of individual attributes on both the intercept and the slope of this relationship. Finally, in this chapter I introduced the three surveys that will be used in the analysis: the Quality of Life Survey, the New Zealand General Social Survey, and Te Kupenga.

Chapter 4 will address The Primary Hypothesis at length, using data from the QoLS. Chapter 4 will also discuss The Parochialism Hypothesis. Chapter 5 will retest The Primary Hypothesis drawing on the NZGSS, and will also explore the mechanisms underlying sensitivity to SWB inequality by addressing The Altruism Hypothesis and The Fairness Hypothesis. Chapter 6 will again retest The Primary Hypothesis, this time using data from Te Kupenga to determine the relevance of the findings for Māori, and using a more comprehensive SWB measure. I will also retest The Fairness Hypothesis and The Altruism Hypothesis in Chapter 6. 


\section{Chapter 4: Are People Sensitive to Wellbeing Inequality Where They Live?}

Limited empirical research to date suggests wellbeing inequality within countries negatively impacts the country's average level of wellbeing. People expressing higher wellbeing live in countries that have a more equal distribution of wellbeing. But how general is this result? Is it likely to apply within countries with as much strength as it does between them? This chapter draws on the New Zealand Quality of Life Survey (QoLS) to address the question of whether the wellbeing of those living within a country are also sensitive to wellbeing inequality in their local area.

The QoLS is conducted biannually as part of the 'Quality of Life Project': a collaboration between a number of New Zealand's city and district councils. While the QoLS has been administered every two years since 2004, changes in data collection methods and the questions asked meant only the 2012 and 2014 datasets are used in the analysis that follows. ${ }^{6}$

I begin by asking whether the average life satisfaction of those living in the six cities available (Auckland, Wellington, Hutt City, Porirua, Christchurch and Dunedin) is negatively related to the inequality of SWB within those cities. I then ask the same question of the electoral wards ${ }^{7}$ within them, and whether the life satisfaction and life satisfaction inequality relationship varies by city. Following the estimation of these ecological models, I estimate the individual models controlling for demographic, attainment-based and subjective characteristics.

\section{1: The Primary Hypothesis - Does Average Wellbeing Decline with the Spread of Wellbeing?}

The following scatterplot (Figure 4.1) of the cities' mean life satisfaction and standard deviation life satisfaction is indeed negative, with a coefficient $(b)$ of -2.554 $(\mathrm{p}<0.05)$ and a standard error $(S E)$ of $0.615 .^{8}$ An increase of standard deviation life

\footnotetext{
${ }^{6}$ The 2010 and 2016 data was also made available to me for analysis. However, a drastic change in survey methodology and question structure between 2010 and 2012 made it impractical to analyse the 2010 survey alongside the others. The life satisfaction question was removed from the 2016 survey, so it could not be used for my analysis.

${ }^{7}$ Electoral wards were used for all of the cities except Auckland, who used community and local boards (which tended to be similar in size to wards). For the sake of simplicity, they will also be referred to as wards throughout this thesis.

${ }^{8}$ The regressions were run with and without frequency weights. The two results were very similar. Only the non-weighted results are presented here.
} 
$41 \mid \mathrm{P}$ a g e

satisfaction in the city is therefore associated with a decline in mean life satisfaction of -2.554 points on the 5-point scale. The $\mathrm{R}^{2}$ is high at 0.812 , indicating life satisfaction inequality in cities accounts for a large portion of the variation in the mean life satisfaction of cities.

Figure 4.1: The relationship between mean and standard deviation life satisfaction by selected cities

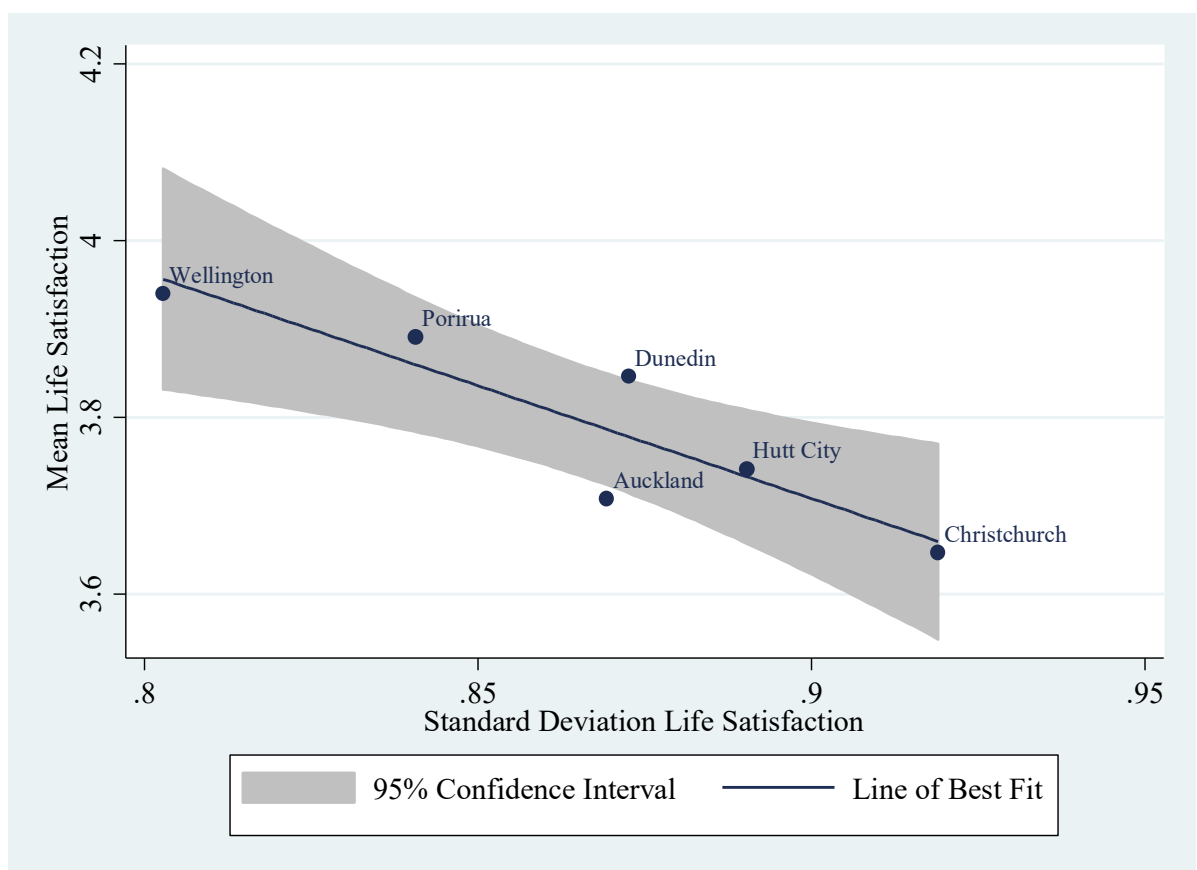

Figure 4.1: QoLS, 2012, New Zealand.

Figure 4.2 explores this relationship at a more local level, plotting the mean and standard deviation of life satisfaction of the various wards in Auckland City. The legend below assigns names to the value labels in the figure. The same negative relationship between the average and the spread of life satisfaction holds across the wards of Auckland. The sensitivity of the average is less marked than in the six-city case above. ${ }^{9}$

The $y$ and $x$ axes in Figure 4.2 indicate there is more variation in the standard deviation of life satisfaction within Auckland city than there is between the various cities of New Zealand, but variation in mean life satisfaction is similar. The magnitude of the coefficient is not as large for the wards within Auckland as it is for the city level, with a one unit increase in standard deviation life satisfaction resulting in only a -0.989 fall in mean life satisfaction $(a=4.561, b=-0.989, S E=0.224, p<0.01)$. A 0.5 unit increase would result in a fall in mean life satisfaction of -0.495 , and a 0.2 unit increase

\footnotetext{
${ }^{9}$ Initially, all of the graphs were produced with consistent scales on the $x$ and $y$ axes. However, this negatively impacted the clarity of many of the graphs. As such, note that the scales on the graphs in this chapter vary.
} 
with a fall of -0.198 . The $\mathrm{R}^{2}$ is 0.466 . Therefore, while my main hypothesis is supported, my hypothesis that sensitivity to wellbeing inequality is greater at smaller scales is not. The sensitivity of average life satisfaction to life satisfaction inequality across wards is weaker than it is at the city level.

\section{Figure 4.2: The relationship between mean and standard deviation life} satisfaction among Auckland wards

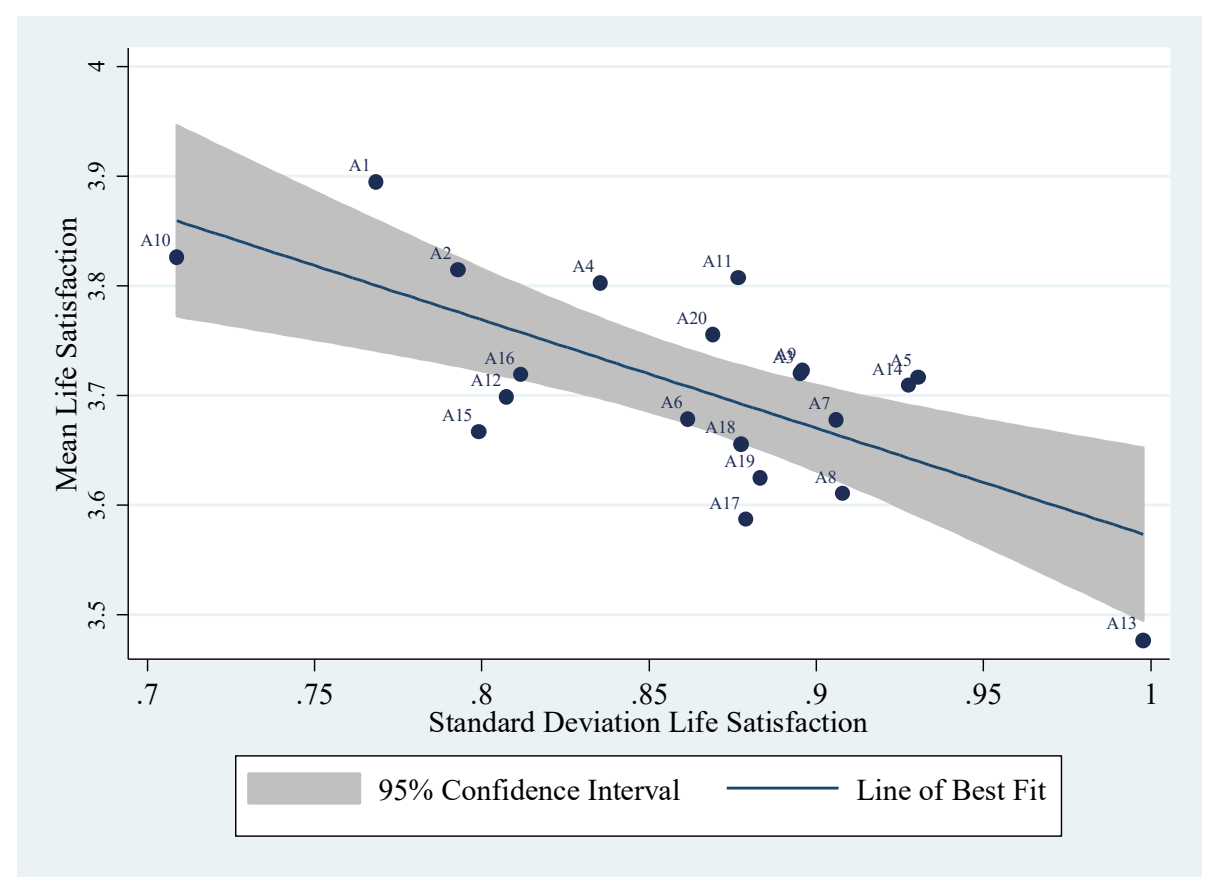

\begin{tabular}{|l|l|l|l|}
\hline A1 & Rodney & A11 & Albert-Eden \\
\hline A2 & Hibiscus and Bays & A12 & Puketapapa \\
\hline A3 & Upper Harbour & A13 & Maungakiekie-Tamaki \\
\hline A4 & Kaipatiki & A14 & Orakei \\
\hline A5 & Devonport-Takapuna & A15 & Howick \\
\hline A6 & Waitakere Ranges & A16 & Otara Papatoetoe \\
\hline A7 & Henderson-Massey & A17 & Mangere-Otahuhu \\
\hline A8 & Whau & A18 & Manurewa \\
\hline A9 & Waitemata & A19 & Papakura \\
\hline A10 & Waiheke and Great Barrier Is. & A20 & Franklin \\
\hline
\end{tabular}

Figure 4.2: QoLS, 2012, New Zealand

Is there a geographic pattern to the scatter in Figure 4.2? Figure 4.3 displays a map of the wards plotted above. At the two extremes of the standard deviation values are Waiheke and the Great Barrier Islands (lowest standard deviation [SD]), and Maungakiekie-Tamaki (highest $S D$ ). The former is on the city outskirts, and the latter is roughly central relative to the other wards. Although some neighbouring wards are similar in terms of life satisfaction and life satisfaction inequality (e.g. A17, A18 and A19), there does not appear to be evidence of a geographic pattern overall. 
Figure 4.3: Map of Auckland wards available for analysis in the Quality of Life Survey

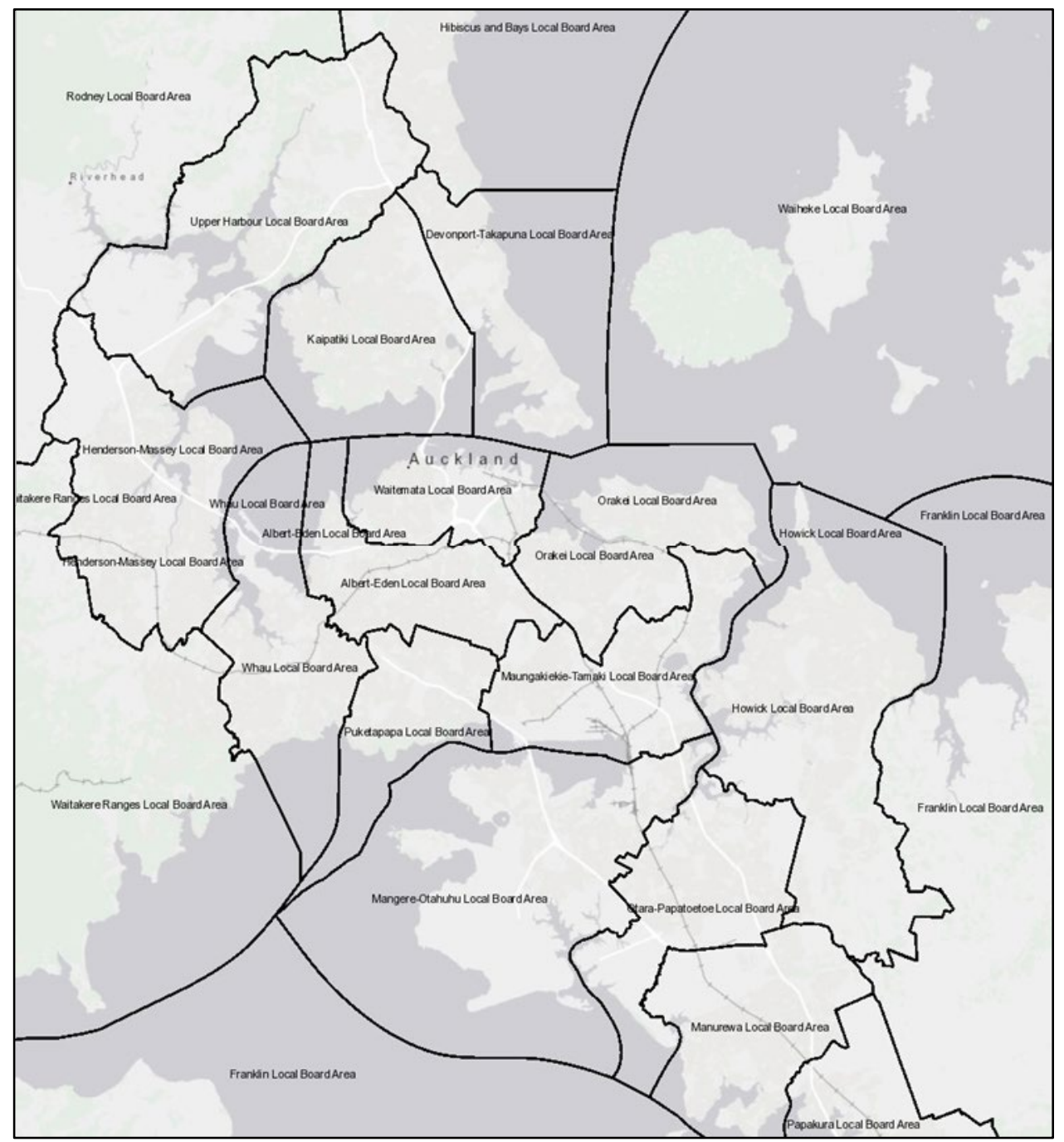

Figure 4.3: Map sourced from the Statistics New Zealand site http://archive.stats.govt.nz/StatsMaps/Home/Boundaries/geographic-boundary-viewer.aspx

Figure 4.4 presents a second within-city test of my hypothesis that wellbeing declines with rising local wellbeing inequality, by plotting mean and the standard deviation of life satisfaction across the different wards within the Wellington metropolitan region (Wellington City, Hutt City and Porirua City). Once again there is a negative relationship between mean and standard deviation wellbeing ( $a=4.935, b=-$ $1.306, S E=0.316, \mathrm{p}<0.01)$. This suggests that ward averages in the Wellington Region are more sensitive to ward inequality than in the case of Auckland. In addition, the $\mathrm{R}^{2}$ value is 0.586 , indicating that more of the variation in mean wellbeing is accounted for by wellbeing inequality in Wellington than in Auckland. However, wards continue to be less sensitive to wellbeing inequality within them than cities are in New Zealand. 
Figure 4.4: The relationship between mean and standard deviation life satisfaction among Wellington Region wards

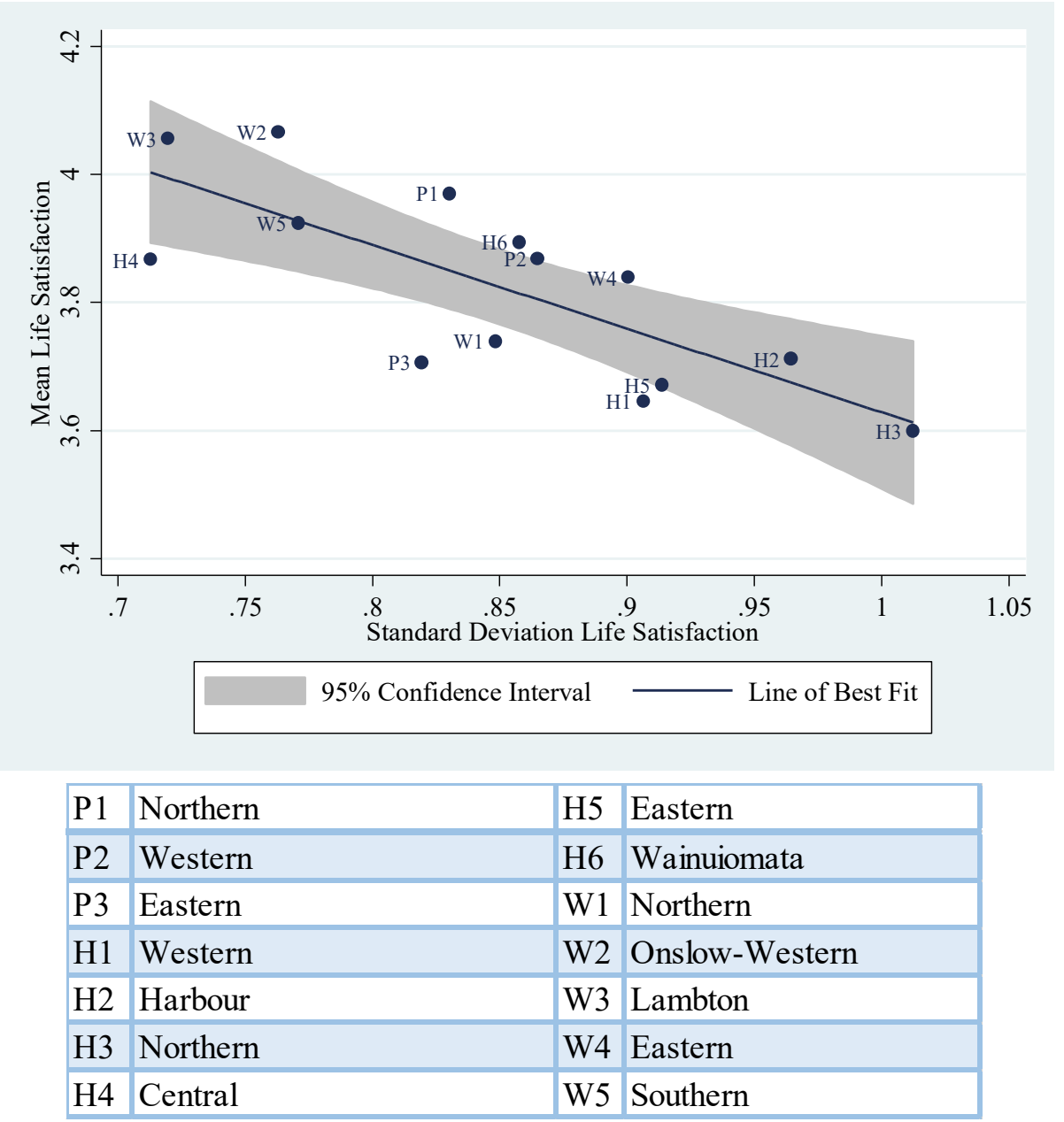

Figure 4.4: QoLS, 2012, New Zealand

Is there a geography to the relationship between SWB and SWB inequality in the Wellington Region? Figure 4.5 displays a map of the wards within each of Wellington, Hutt City and Porirua. The difference between Hutt City and the other two cities is the starkest. The Northern ward (H3) has the highest standard deviation of life satisfaction out of the wards in the Wellington Region. Likewise, the Western, Harbour and Eastern wards (H1, $\mathrm{H} 2$ and $\mathrm{H} 5$ respectively) all have standard deviation values higher than any of the Wellington or Porirua wards, and mean life satisfactions lower than most of Wellington and Porirua. In contrast, the Central ward of Hutt City has the lowest standard deviation life satisfaction in the Wellington Region, but still has a lower mean than all but two of the Wellington and Porirua wards - potentially owing to the higher inequality in wellbeing in wards surrounding it on all sides.

Within Wellington city, the neighbouring Onslow-Western and Lambton wards are both more satisfied and more equal in that satisfaction than the majority of other 
$\mathbf{4 5} \mid \mathrm{P}$ a g e

Wellington Region wards. A geography of sensitivity to wellbeing inequality is difficult to identify within Porirua given it only contains three wards.

Figure 4.5: Map of Wellington Region wards available for analysis in the Quality of Life Survey

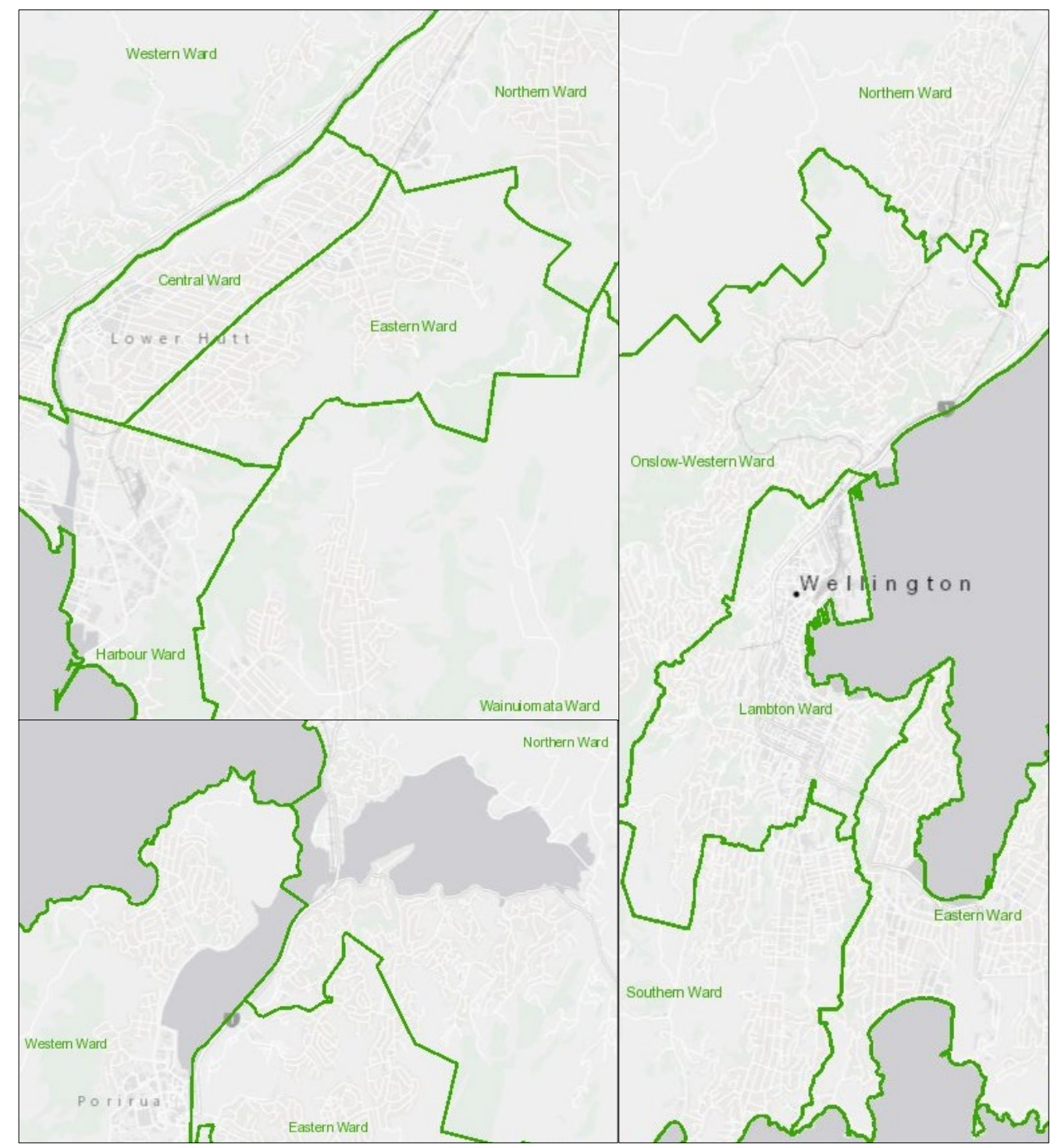

Figure 4.5: Maps sourced from the Statistics New Zealand site http://archive.stats.govt.nz/StatsMaps/Home/Boundaries/geographic-boundary-viewer.aspx

Figure 4.6 displays the same relationship for the wards in Christchurch. ${ }^{10}$ Wards in Christchurch demonstrate the second strongest negative relationship between mean life satisfaction and life satisfaction inequality out of the cities analysed thus far $(a=4.804, b=-1.277, S E=0.224, p<0.01)$. The $\mathrm{R}^{2}$ value is high at 0.891 .

\footnotetext{
10 The Banks Peninsula ward was available for analysis, but was excluded due to having a small sample size of only 12 .
} 
Figure 4.7 displays a map of the Christchurch wards analysed to assist in the identification of geographic patterns. The two extremes of standard deviation life satisfaction on the plot are Riccarton-Wigram (C4) at the lower end and BurwoodPegasus (C3) at the upper end. While these wards are on opposite ends of the city, there does not appear to be a discernible geography to the relationship in Figure 4.6.

Figure 4.6: The relationship between mean and standard deviation life satisfaction among Christchurch wards

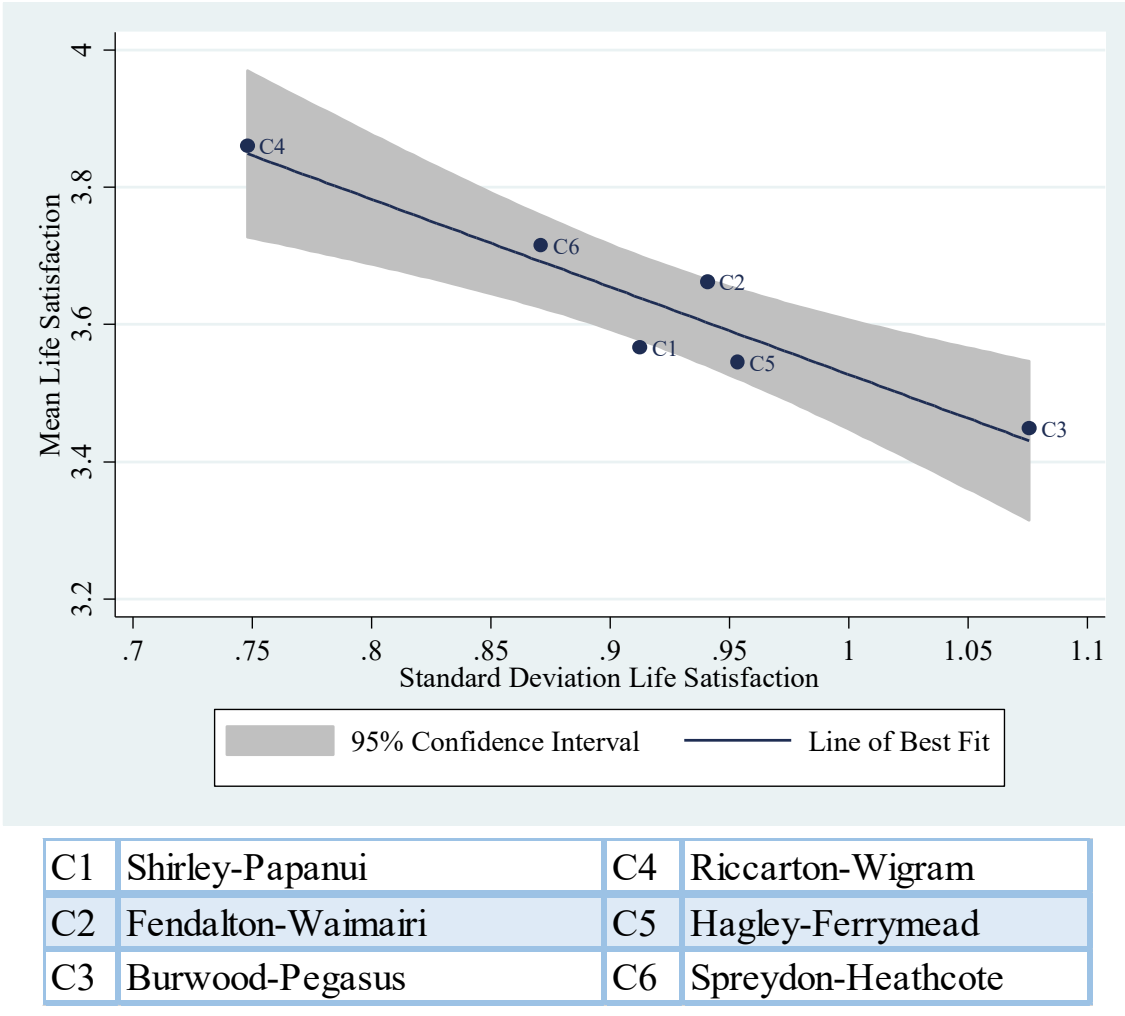

Figure 4.6: QoLS, 2012, New Zealand

Figure 4.7: Map of Christchurch wards available for analysis in the Quality of Life Survey

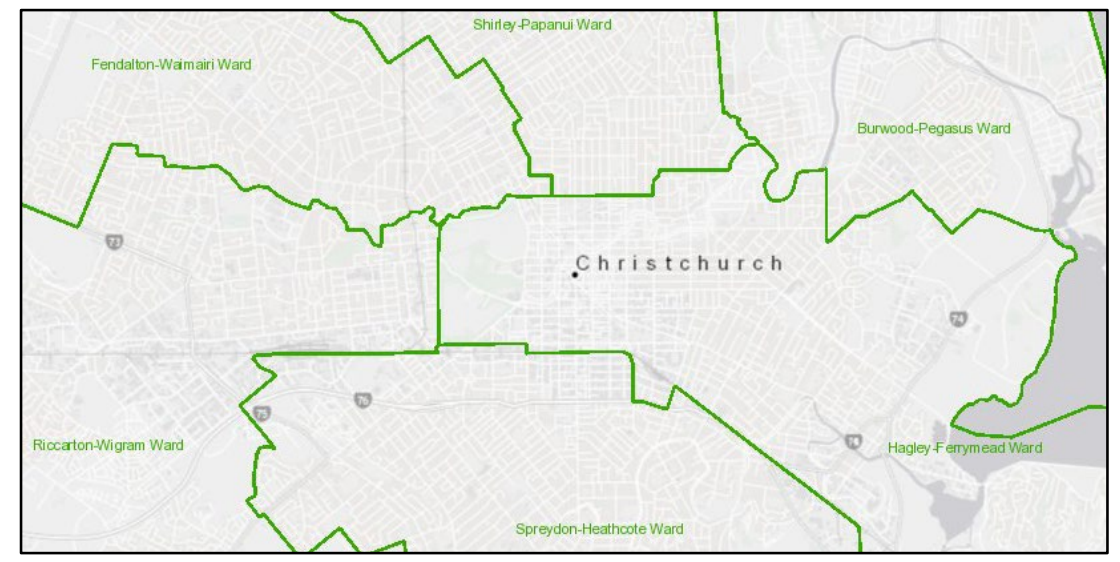

Figure 4.7: Map sourced from the Statistics New Zealand site $\underline{\text { http://archive.stats.govt.nz/StatsMaps/Home/Boundaries/geographic-boundary- }}$ viewer.aspx 
Of all the cities, Dunedin demonstrates the weakest relationship between the mean and standard deviation life satisfaction of its wards. A one unit increase in a ward's standard deviation is associated with a -0.737 fall in the mean life satisfaction of the ward $(a=4.491, b=-0.737, S E=0.179, p<0.05)$. A 0.5 unit increase in a ward's standard deviation life satisfaction is associated with a fall in mean life satisfaction of 0.369 , and a 0.2 unit increase is associated with a fall of -0.147 . The $\mathrm{R}^{2}$ is 0.809 .

According to the map in Figure 4.9, there does not appear to be a geography to the relationship in Figure 4.8. The ward with the highest standard deviation value is South Dunedin, while the one with the lowest is neighbouring Green Island-Saddle Hill ward.

Figure 4.8: The relationship between mean and standard deviation life satisfaction among Dunedin wards

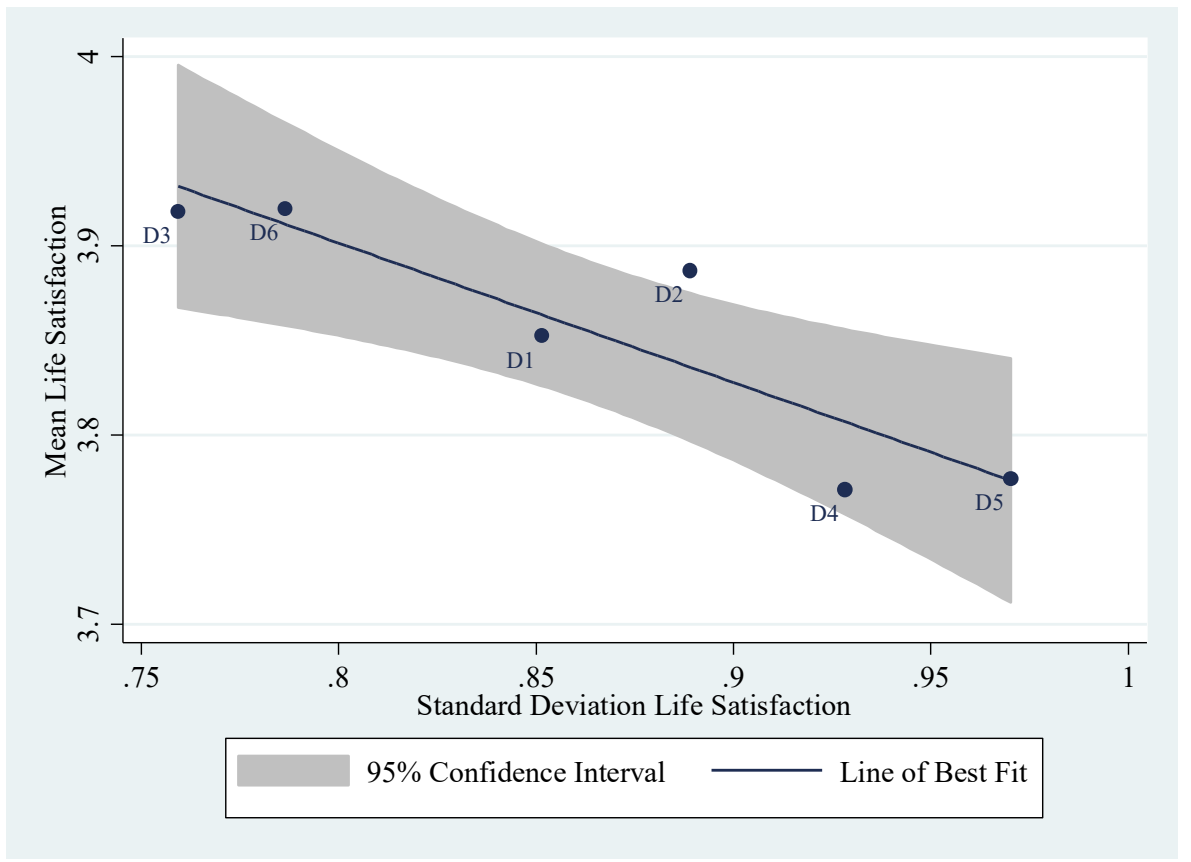

\begin{tabular}{|l|l|l|l|}
\hline D1 & Mosgiel-Taieri & D4 & Hills \\
\hline D2 & Waikouaiti Coast-Chalmers & D5 & South Dunedin \\
\hline D3 & Green Island-Saddle Hill & D6 & Cargill \\
\hline
\end{tabular}

Figure 4.8: QoLS, 2012, New Zealand 
Figure 4.9: Map of Dunedin wards available for analysis in the Quality of Life Survey

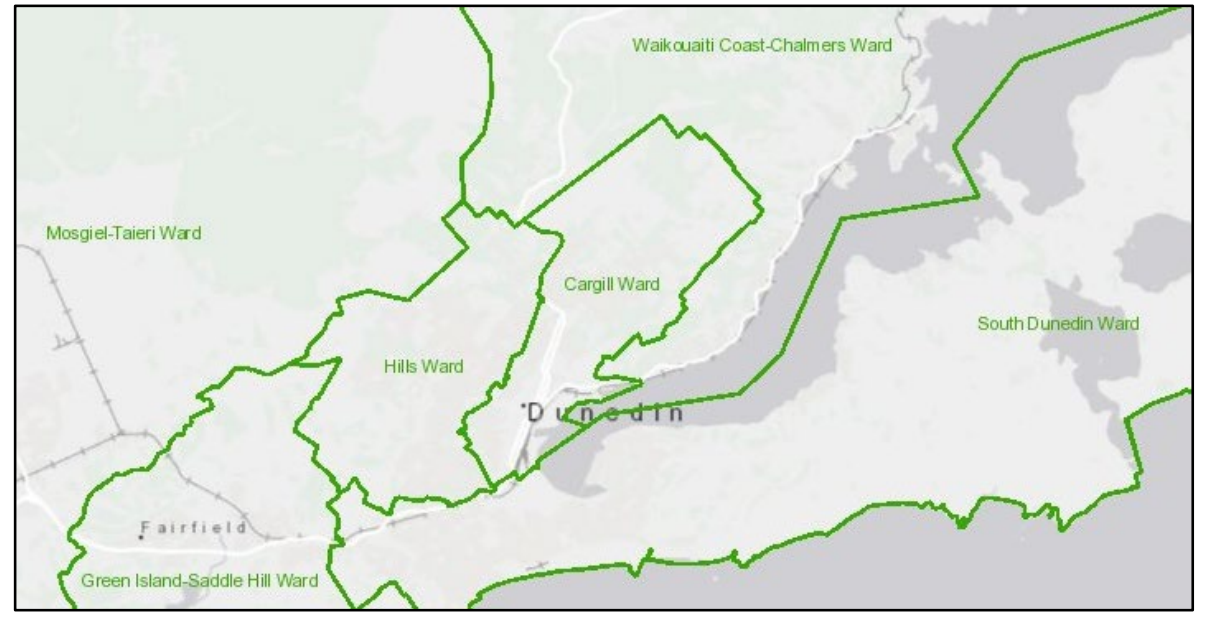

Figure 4.9: Map sourced from the Statistics New Zealand site http://archive.stats.govt.nz/StatsMaps/Home/Boundaries/geographic-boundary-viewer.aspx

I have presented regressions of each city separately using the wards within them, but the pattern for the wards throughout the entirety of New Zealand has not yet been examined. Doing so provides a more accurate picture of the country-wide relationship between life satisfaction and life satisfaction inequality than the analysis using the six cities. Figure 4.10 presents the relationship between mean and standard deviation life satisfaction among all of the wards in the QoLS for 2012.

In line with my primary hypothesis, there is a negative relationship between the wards' means and their standard deviation of life satisfaction. On average, every one unit increase in the standard deviation life satisfaction of a ward is associated with a decrease of -1.233 in the mean life satisfaction of that ward on a 5-point scale, while a 0.5 unit increase is associated with a decrease of -0.617 , and a 0.2 unit increase with a decrease of $-0.247(a=4.820, b=-1.233, S E=0.178, p<0.001)$. The $\mathrm{R}^{2}$ for the regression here is 0.523 .

So far there is no evidence indicating the relationship between mean and standard deviation life satisfaction is non-linear, in keeping with the observations of researchers at the international level (Delhey, 2004; Ott, 2005). 
Figure 4.10: The relationship between mean and standard deviation life satisfaction among New Zealand wards

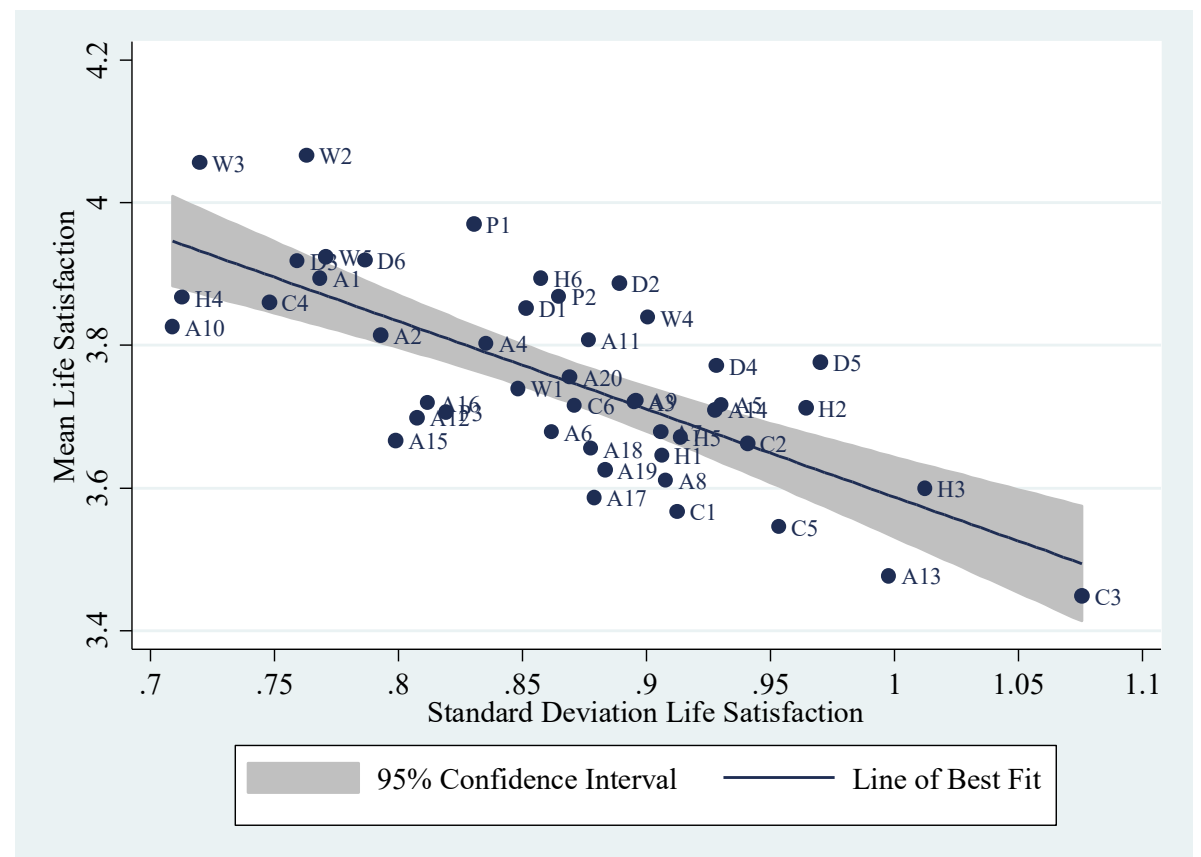

Figure 4.10: QoLS, 2012, New Zealand

However, so far I have only examined the relationship using the 2012 data of the QoLS. It is worth utilising the 2014 data to see how stable the relationships are across adjoining surveys.

Figure 4.11 presents a graph of the same wards from both 2012 and 2014. ${ }^{11}$ Life satisfaction in 2012 is represented by the darker shade of blue, with filled-in dots and a solid line, while life satisfaction in 2014 is represented by a lighter shade of blue with hollow dots and a dashed line. Note that the confidence intervals have been made transparent and are bordered by only a grey outline in the following figures.

Figure 4.11 shows that while relationship between mean and standard deviation life satisfaction is negative in both 2012 and 2014, it appears to be stronger in 2012 than in 2014. Whereas in 2012 the regression of the relationship yields a coefficient of $1.233(p<0.001)$ and an $\mathrm{R}^{2}$ of 0.523 , in 2014 we get a much smaller coefficient of only $-0.594(a=4.315, b=-0.594, S E=0.210 p<0.01)$, and an $\mathrm{R}^{2}$ of 0.154 . Evidently, there is a considerable difference between the years regarding how mean life satisfaction changes in response to standard deviation life satisfaction. The difference between

\footnotetext{
${ }^{11}$ Residual plots were created of the observations in Figure 4.6 and Figure 4.7. The plots indicate that the points are randomly distributed in each case.
} 
$\mathbf{5 0} \mid \mathrm{P}$ a g e

years warrants further exploration, as well as in the other years for which the data is available. While I can address the former, an exploration of the latter falls outside of the scope of this particular thesis.

\section{Figure 4.11: The relationship between mean and standard deviation life} satisfaction among New Zealand wards, 2012 and 2014

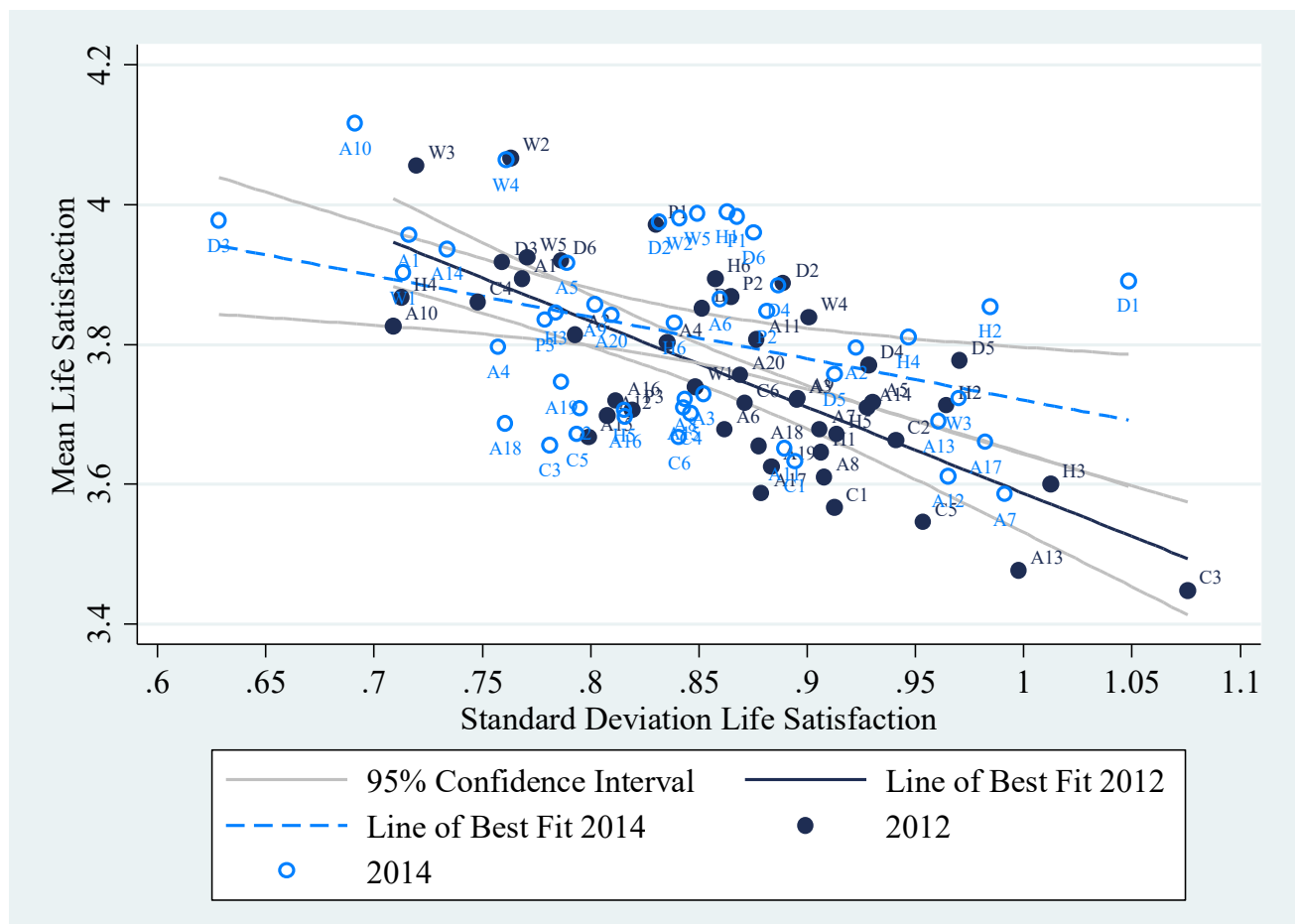

Figure 4.11: QoLS, 2012 and 2014, New Zealand

I compare 2012 and 2014 using the city variable by plotting Figure 4.12. Figure 4.12 visualises how the relationship between the mean and inequality in life satisfaction of the wards has changed in each city between 2012 and 2014. Note that while the order of most satisfied to least satisfied city is completely consistent between the two years, the order of most equal to most unequal changes for every city except Hutt City, which is second most unequal in both years.

While the mean and standard deviation life satisfaction of Porirua, Hutt City, Dunedin and Auckland have changed relatively little between the surveys, large differences are apparent in Wellington, and even larger ones in Christchurch. While Wellington is the most satisfied and most equal city in 2012, it is the most satisfied but only third most equal city in 2014. In contrast, Christchurch moves from being the most dissatisfied and most unequal city in 2012 to the most dissatisfied and most equal city 
in 2014. Note that while the magnitude of the coefficient in Figure 4.12 is large and positive at 1.602 , the effect is not statistically significant $(p=0.389)$.

Figure 4.12: The relationship between mean and standard deviation life satisfaction by selected cities, 2012 and 2014

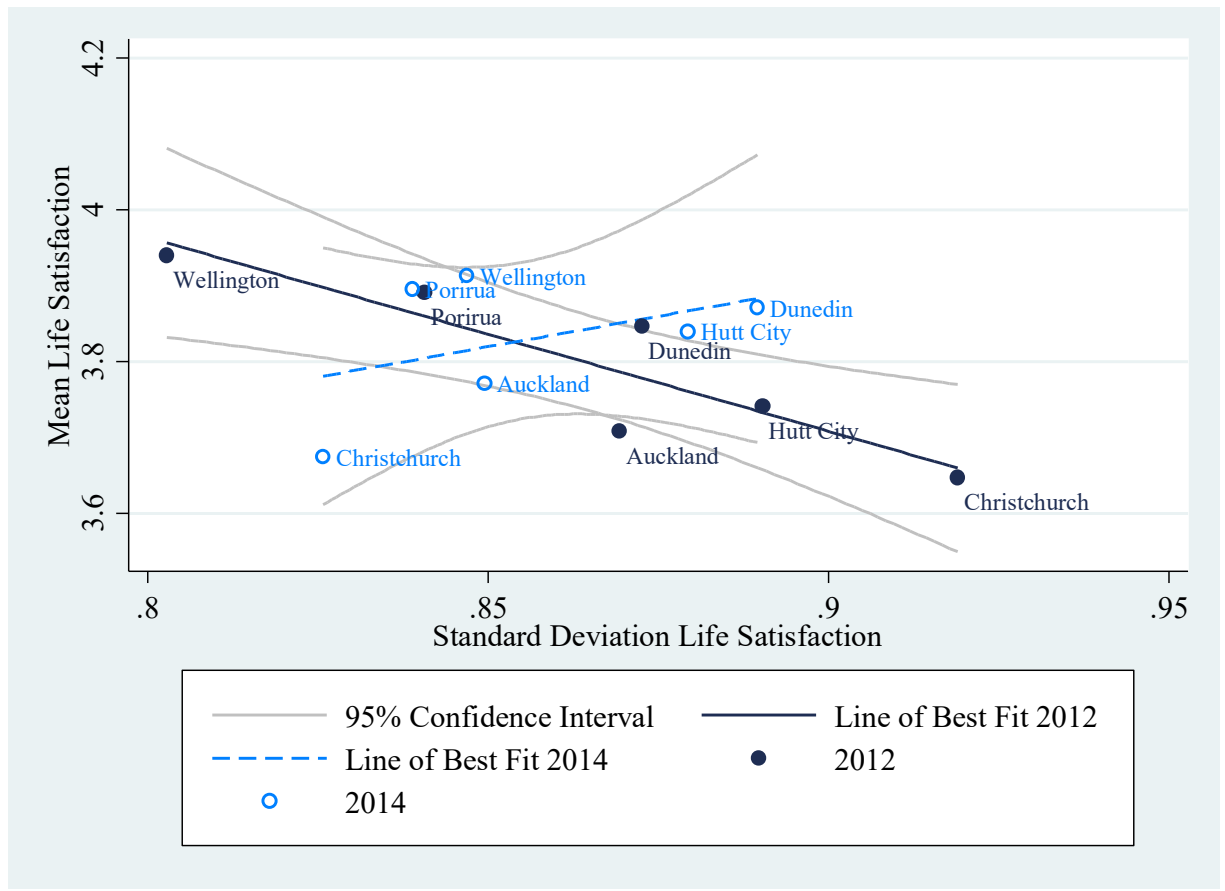

Figure 4.12: QoLS, 2012 and 2014, New Zealand

What could have caused this large decrease in life satisfaction inequality and simultaneous stagnancy in average life satisfaction within Christchurch? It is possible that Christchurch residents felt the repercussions of the 2011 earthquake in terms of their average wellbeing, but that the distribution of wellbeing had become more equal as residents became more community-oriented and inequality in life circumstances began to be redressed. It is also possible that those at the lower or upper ends of the wellbeing distribution moved away from Christchurch after the earthquake, leaving the majority of moderately satisfied individuals behind. ${ }^{12}$

Until I examine the relationship within each city, I cannot be sure that it is solely Christchurch showing an unexpected pattern. Figures 4.13, 4.14, 4.15, and 4.16 replicate the scatter plots for the wards within each of Auckland, the Wellington Region, Christchurch, and Dunedin respectively, except with both 2012 and 2014 plotted.

\footnotetext{
12 There may be a compounding collection issue as well. In 2012, all of the cities met their target responses for data collection, whereas in 2014 Christchurch was the only city to fall short, collecting 488 out of the target 500 responses. More information on target responses and the data collection process is available in Appendix A.
} 
Figure 4.13 indicates Auckland wards demonstrate a similar relationship between mean and standard deviation life satisfaction in both 2012 and 2014. Indeed, the magnitude and significance of the coefficient is stronger in 2014 at $-1.130(a=4.727$, $b=-1.130, S E=0.227, p<0.001)$ than in 2012 at $-0.989(\mathrm{p}<0.01)$. The $\mathrm{R}^{2}$ in 2014 is also higher at 0.580 , relative to 0.466 in 2012 .

Figure 4.14 compares wards in the Wellington Region in 2014 and 2012. In 2014 the magnitude of the correlation coefficient is much lower at -0.435 , and is statistically not significant $(p=0.259)$. OLS regressions of the relationships displayed in Figure 4.15 and 4.16 indicate that the 2014 relationship is also not statistically significant for Christchurch ( $p=0.414$ ) or Dunedin $(p=0.311)$. So while the relationship between mean and standard deviation life satisfaction is significant in both years for Auckland wards and New Zealand wards overall, it is not significant in the wards of the Wellington Region, Christchurch or Dunedin in 2014.

Figure 4.13: The relationship between mean and standard deviation life satisfaction among Auckland wards, 2012 and 2014

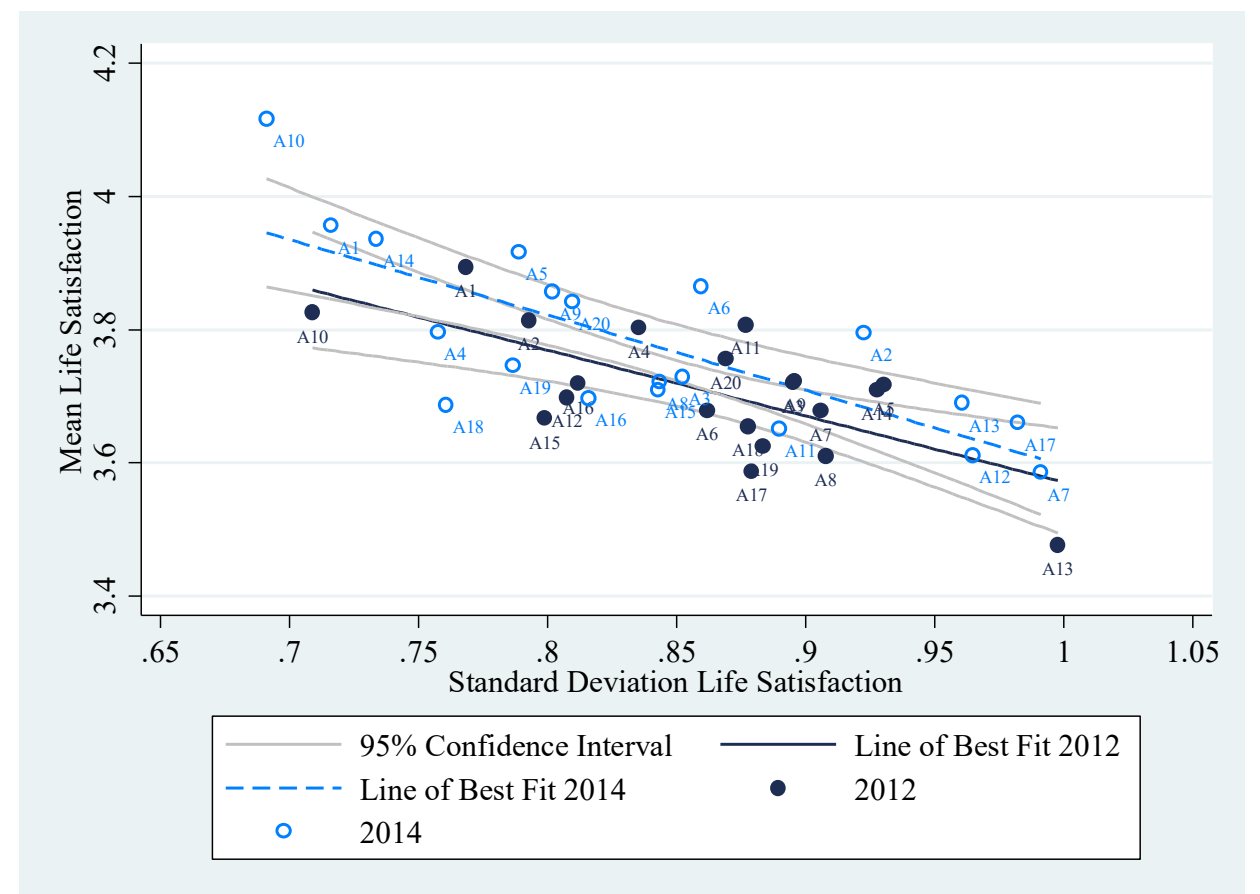

Figure 4.13: QoLS, 2012 and 2014, New Zealand 
Figure 4.14: The relationship between mean and standard deviation life satisfaction among Wellington Region wards, 2012 and 2014

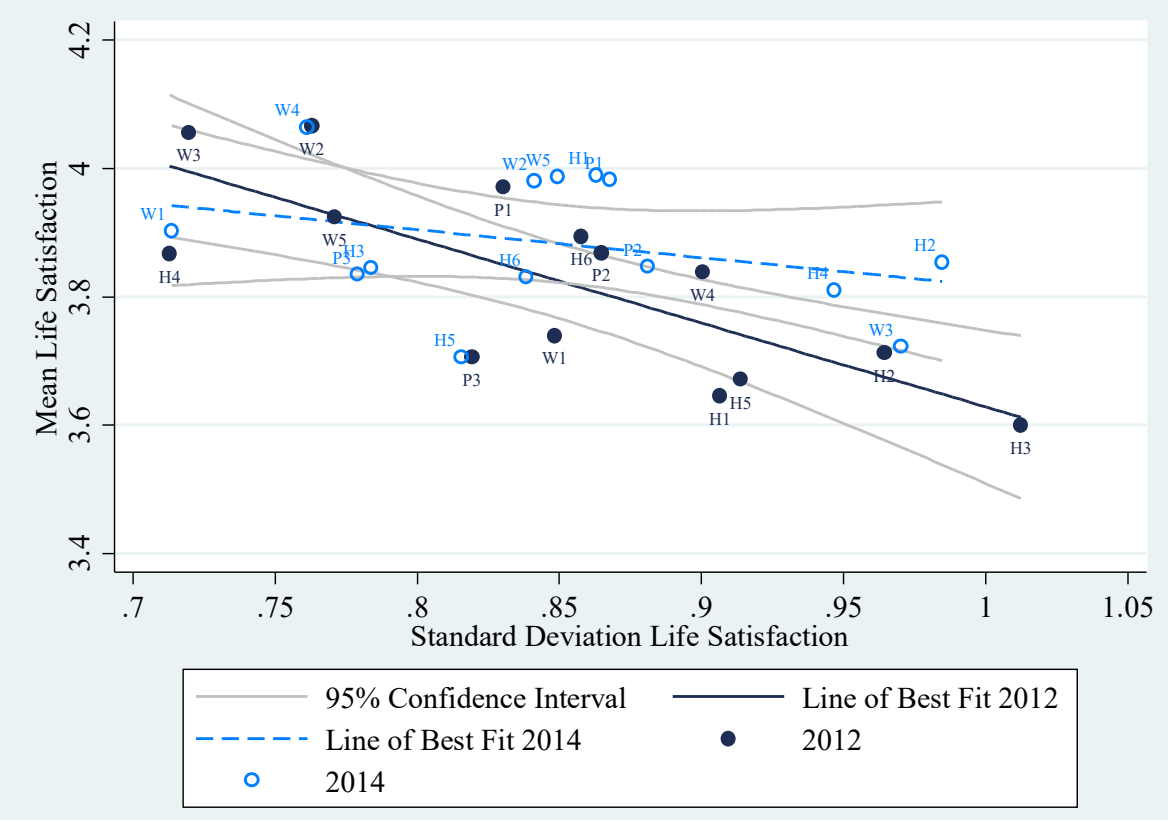

Figure 4.14: QoLS, 2012 and 2014, New Zealand

Figure 4.15: The relationship between mean and standard deviation life satisfaction among Christchurch wards, 2012 and 2014

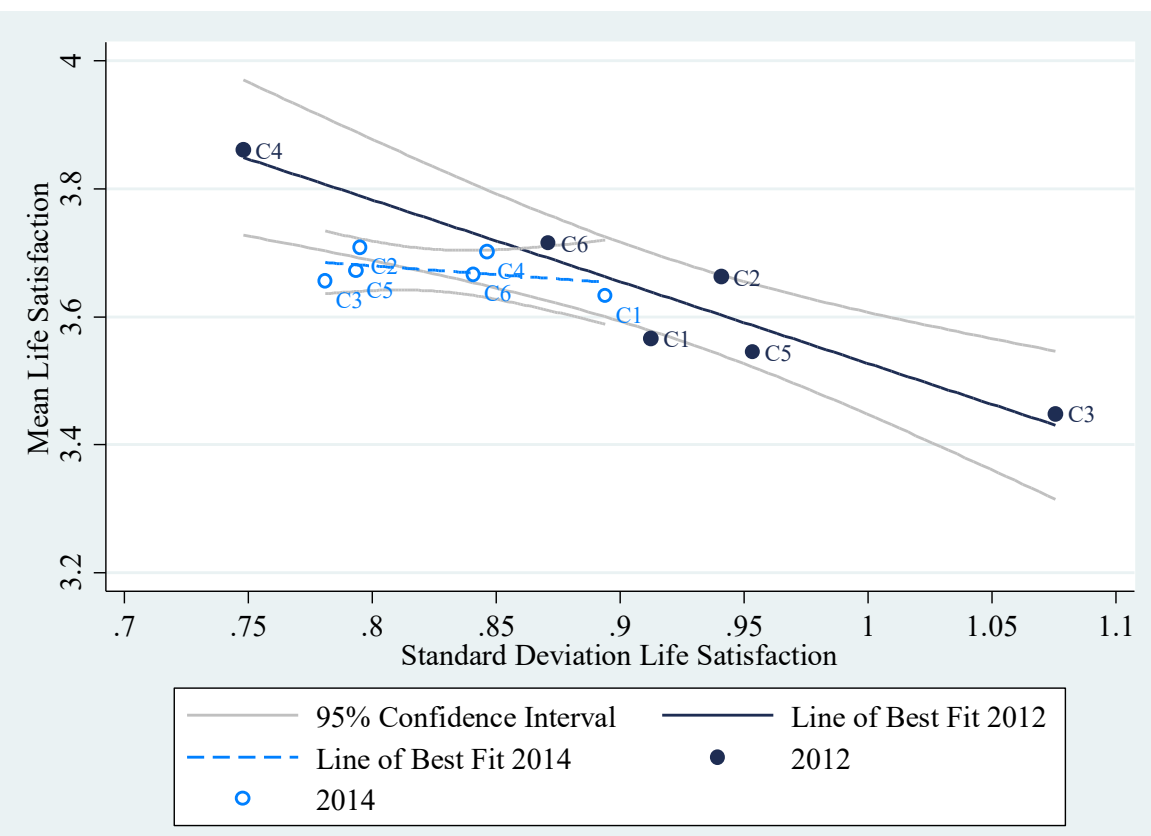

Figure 4.15: QoLS, 2012 and 2014, New Zealand 
Figure 4.16: The relationship between mean and standard deviation life satisfaction among Dunedin wards, 2012 and 2014

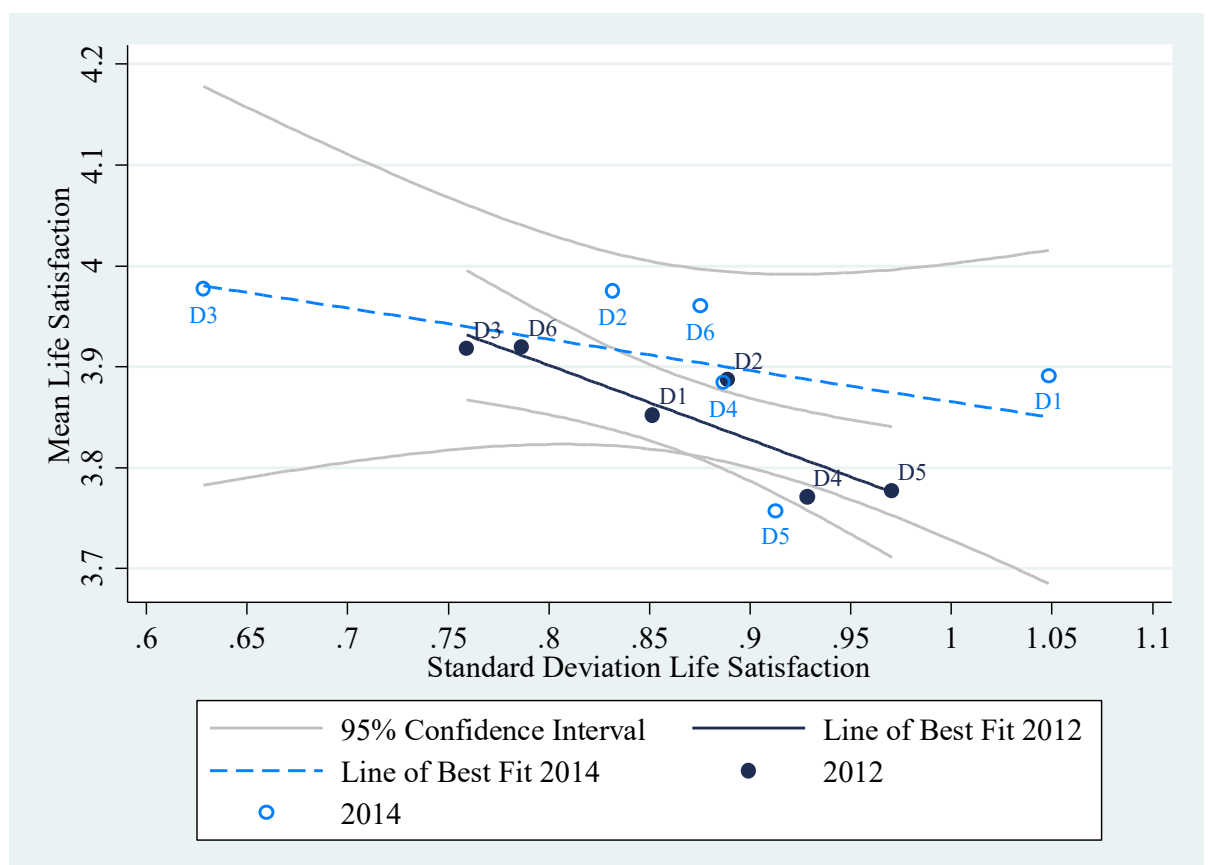

Figure 4.16: QoLS, 2012 and 2014, New Zealand

Note the extreme values of the Dunedin wards D3 and D1 in Figure 4.11 and Figure 4.16. These wards have the lowest and highest standard deviation values respectively for the 2014 data. The next step is to check whether any of the remaining wards, such as these Dunedin ones, are likely to be outliers compared to the remainder of the dataset. Figure 4.17 plots the distribution of standard deviation life satisfaction values in New Zealand wards.

There is one outlier in 2012 (C3: Burwood-Pegasus) and two in 2014 (D3: Green Island-Saddle Hill at the lower end, and D1: Mosgiel-Taieri at the upper end). Excluding the outliers from analysis has an impact on the results. In 2012 for all New Zealand wards collectively, a one unit increase in standard deviation life satisfaction is now associated with a drop of -1.194 in mean life satisfaction, while a 0.5 unit increase is associated with a drop of -0.597 , and a 0.2 unit increase with a drop of -0.239 ( $a=4.787, b=-1.194, S E=0.195, p<0.001)$. The $\mathrm{R}^{2}$ drops to 0.466 . The effect of life satisfaction inequality on mean life satisfaction in 2014 wards gets stronger after outliers are removed, rising to $-0.724(a=4.419, b=-0.724, S E=0.241, p<0.01)$. The $\mathrm{R}^{2}$ for 2014 increases accordingly to 0.177 . Evidently, running the regressions without the outliers has reduced the gap between the two years, but has not fully explained the 
difference between them. I will move to analysing the relationship using individual data and controls to explore this difference.

\section{Figure 4.17: The spread of standard deviation life satisfaction by year}

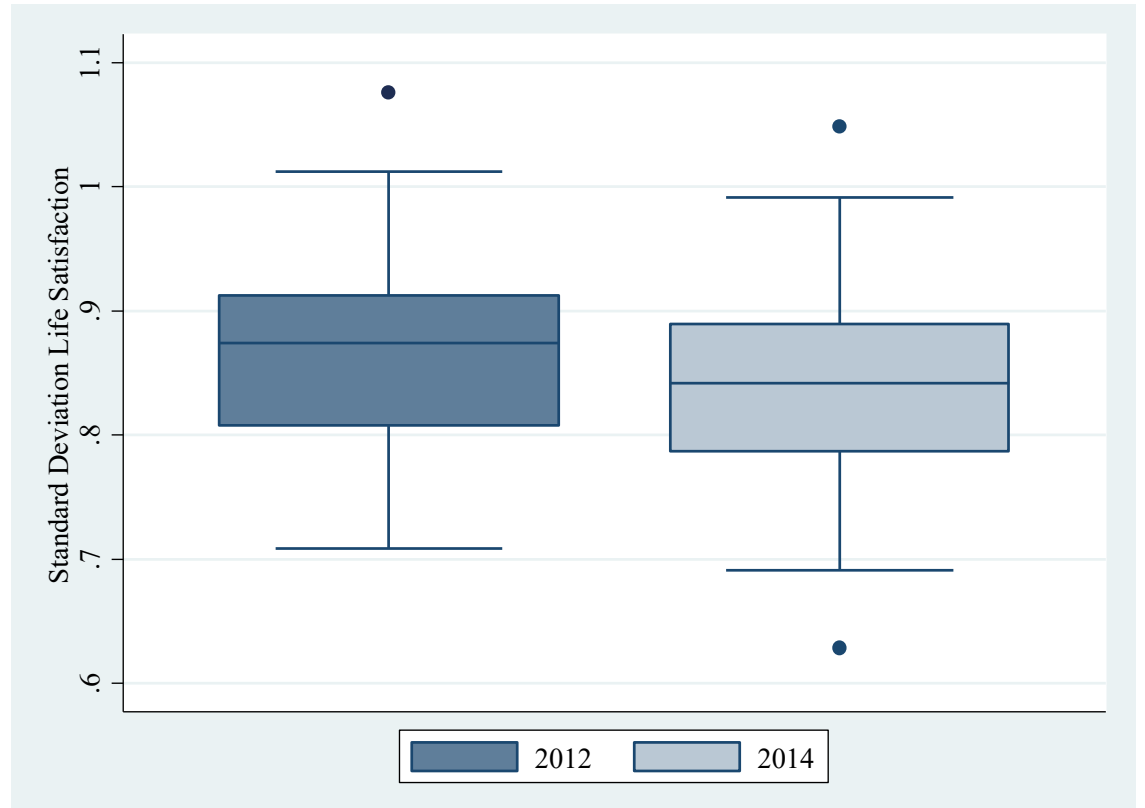

Figure 4.17: QoLS, 2012 and 2014, New Zealand

\section{2: The Primary Hypothesis - Does Individual Wellbeing Decline with the Spread of Wellbeing?}

Tables 4.1 and 4.2 provide eight models of individual life satisfaction against standard deviation life satisfaction in the individual's ward for 2012 and 2014 respectively, without the outliers identified in Figure 4.17. I apply Equation 8 from Chapter 3 adjusting for the clustering of individuals within wards.

With each model, a new control variable is included to test for its impact on the relationship between SWB inequality and individual SWB, in both substantive and statistical terms. A city control is introduced first, followed by demographic controls (sex, age group, and ethnicity), and the achievement controls (employment, education, and income). While subjective controls for health, sense of community, and social isolation are introduced last and will be discussed, they are not reported on in the estimates tables owing to their greater likelihood of an endogenous relationship with life satisfaction. 
Table 4.1: Models of individual life satisfaction against standard deviation life satisfaction of wards, 2012 (cluster-controlled)

\begin{tabular}{|c|c|c|c|c|c|c|c|c|}
\hline & Model 1 & Model 2 & Model 3 & Model 4 & Model 5 & Model 6 & Model 7 & Model 8 \\
\hline SD Life Satisfaction & $-1.255^{* * *}$ & $-1.048 * * *$ & $-1.047 * * *$ & $-1.023 * * *$ & $-.992 * * *$ & $-.937 * * *$ & $-.948 * * *$ & $-.918 * * *$ \\
\hline \multicolumn{9}{|l|}{ City (Base Auckland) } \\
\hline Porirua & & $.151 *$ & $.149^{*}$ & $.138^{*}$ & $.115^{*}$ & $.0994 * *$ & $.1 * *$ & $.0886^{* *}$ \\
\hline Hutt City & & .0505 & .0495 & .0236 & .00191 & -.00365 & .00261 & .0475 \\
\hline Wellington & & $.155 * * *$ & $.153 * * *$ & $.155^{* *}$ & $.133 * *$ & $.131 * * *$ & $.11 * *$ & $.112 * * *$ \\
\hline Christchurch & & -.0137 & -.0107 & -.0328 & $-.0669 *$ & $-.0859 * *$ & $-.081 *$ & $-.0823 *$ \\
\hline Dunedin & & $.143 * * *$ & $.142 * * *$ & $.131 * * *$ & $.0779 * * *$ & $.0607 * *$ & $.0628 * *$ & $.0975 * *$ \\
\hline \multicolumn{9}{|l|}{ Sex (Base Female) } \\
\hline Male & & & $-.0666 * *$ & $-.0849 * *$ & $-.0815 * *$ & $-.0954 * *$ & $-.091 * *$ & $-.141 * * *$ \\
\hline \multicolumn{9}{|l|}{ Age Group, Decades } \\
\hline Younger than 20 & & & & -.00975 & -.0082 & .0928 & .128 & $.269 * *$ \\
\hline $30-39$ & & & & .0556 & .0599 & .0301 & .0129 & .0256 \\
\hline \multicolumn{9}{|l|}{ Base: 40-49 } \\
\hline $50-59$ & & & & .068 & .0616 & .0437 & .0443 & .0533 \\
\hline $60+$ & & & & $.236 * * *$ & $.217 * * *$ & $.181 * * *$ & $.183 * * *$ & $.209 * * *$ \\
\hline New Zealand European & & & & & $.18 * * *$ & $.142 * * *$ & $.149 * * *$ & $.0935^{* *}$ \\
\hline $\begin{array}{r}\text { Employment Status (Base } \\
\text { Full Time) }\end{array}$ & & & & & & & & \\
\hline
\end{tabular}




\begin{tabular}{|c|c|c|c|c|c|c|c|c|}
\hline Part Time & & & & & & -.0469 & -.0404 & $.165^{* * *}$ \\
\hline $\begin{array}{r}\text { Unemployed and looking } \\
\text { for work }\end{array}$ & & & & & & $-.548 * * *$ & $-.529 * * *$ & $-.331 * * *$ \\
\hline $\begin{array}{r}\text { Unemployed and not } \\
\text { looking }\end{array}$ & & & & & & -.0276 & -.00391 & $.218^{* * *}$ \\
\hline University Educated & & & & & & & $.112^{* * *}$ & .0324 \\
\hline Personal Income & & & & & & & & \\
\hline Loss/No Income & & & & & & & & -.174 \\
\hline Less than $\$ 10,000$ & & & & & & & & $-.218 * *$ \\
\hline$\$ 10,001-\$ 20,000$ & & & & & & & & $-.233 * * *$ \\
\hline$\$ 20,001-\$ 30,000$ & & & & & & & & $-.222 * * *$ \\
\hline$\$ 30,001-\$ 40,000$ & & & & & & & & -.108 \\
\hline Base: $\$ 40,001-\$ 50,000$ & & & & & & & & \\
\hline$\$ 50,001-\$ 60,000$ & & & & & & & & .0957 \\
\hline$\$ 60,001-\$ 70,000$ & & & & & & & & $.138 *$ \\
\hline$\$ 70,001-\$ 100,000$ & & & & & & & & $.195^{* *}$ \\
\hline More than $\$ 100,000$ & & & & & & & & $.371 * * *$ \\
\hline Constant & $4.84 * * *$ & $4.62 * * *$ & $4.65^{* * *}$ & $4.56^{* * *}$ & $4.43 * * *$ & $4.49 * * *$ & $4.45^{* * *}$ & $4.39 * * *$ \\
\hline $\mathrm{N}$ & 5036 & 5036 & 5036 & 5036 & 5036 & 4714 & 4601 & 3845 \\
\hline $\mathrm{r} 2$ & .0105 & .0163 & .0178 & .0344 & .0424 & .06 & .0639 & .093 \\
\hline r2_a & .0103 & .0152 & .0164 & .0321 & .0399 & .0568 & .0604 & .0868 \\
\hline rmse & .864 & .861 & .861 & .854 & .851 & .834 & .833 & .826 \\
\hline 11 & -6406 & -6391 & -6387 & -6344 & -6323 & -5826 & -5678 & -4709 \\
\hline
\end{tabular}


Table 4.2: Models of individual life satisfaction against standard deviation life satisfaction of wards, 2014 (cluster-controlled)

\begin{tabular}{|c|c|c|c|c|c|c|c|c|}
\hline & Model 1 & Model 2 & Model 3 & Model 4 & Model 5 & Model 6 & Model 7 & Model 8 \\
\hline SD Life Satisfaction & $-.704 * * *$ & $-.812 * * *$ & $-.800 * * *$ & $-.783 * * *$ & $-.748 * * *$ & $-.672 * * *$ & $-.715 * * *$ & $-.900 * * *$ \\
\hline \multicolumn{9}{|l|}{ City (Base Auckland) } \\
\hline Porirua & & .122 & .121 & .119 & $.113 *$ & .0992 & $.112 *$ & $.102 *$ \\
\hline Hutt City & & .0981 & .0995 & .0937 & .0743 & .0682 & $.0806^{*}$ & $.0915^{*}$ \\
\hline Wellington & & $.138 * *$ & $.138 * *$ & $.153 * *$ & $.130 * *$ & $.125^{*}$ & $.112^{*}$ & $.0982 *$ \\
\hline Christchurch & & $-.106 * * *$ & $-.104 * * *$ & $-.106 * * *$ & $-.130 * * *$ & $-.159 * * *$ & $-.149 * * *$ & $-.124 * * *$ \\
\hline Dunedin & & $.128 * *$ & $.129 * *$ & $.123 * *$ & $.0877^{*}$ & .0782 & .0714 & $.0992 * *$ \\
\hline \multicolumn{9}{|l|}{ Sex (Base Female) } \\
\hline Male & & & $-.0525^{*}$ & $-.0634 * *$ & $-.0623 * *$ & $-.0633 *$ & $-.0548 *$ & $-.0805 * *$ \\
\hline \multicolumn{9}{|l|}{ Age Group, Decades } \\
\hline Younger than 20 & & & & -.0373 & -.0209 & .095 & $.147^{*}$ & .151 \\
\hline $20-29$ & & & & -.0837 & -.0858 & -.0542 & -.0511 & .0523 \\
\hline $30-39$ & & & & .0172 & .0278 & .007 & -.00648 & -.00491 \\
\hline \multicolumn{9}{|l|}{ Base: $40-49$} \\
\hline $50-59$ & & & & .0358 & .0348 & .0217 & .0215 & -.00016 \\
\hline $60+$ & & & & $.219 * * *$ & $.208 * * *$ & $.189 * * *$ & $.203 * * *$ & $.233 * * *$ \\
\hline New Zealand European & & & & & $.155 * * *$ & $.131 * * *$ & $.141 * * *$ & $.114 * *$ \\
\hline \multicolumn{9}{|l|}{$\begin{array}{r}\text { Employment Status } \\
\text { (Base Full Time) }\end{array}$} \\
\hline Part Time & & & & & & -.0523 & -.0473 & $.103^{*}$ \\
\hline
\end{tabular}




\begin{tabular}{|c|c|c|c|c|c|c|c|c|}
\hline $\begin{array}{r}\text { Unemployed and looking } \\
\text { for work }\end{array}$ & & & & & & $-.424 * * *$ & $-.406^{* * *}$ & $-.298 * * *$ \\
\hline $\begin{array}{r}\text { Unemployed and not } \\
\text { looking }\end{array}$ & & & & & & -.0118 & -.00187 & $.171^{* * *}$ \\
\hline University Educated & & & & & & & $.12 * * *$ & .0307 \\
\hline & & & & & & & & \\
\hline Personal Income & & & & & & & & \\
\hline Loss/No Income & & & & & & & & -.0208 \\
\hline Less than $\$ 10,000$ & & & & & & & & -.0994 \\
\hline$\$ 10,001-\$ 20,000$ & & & & & & & & $-.191 * *$ \\
\hline$\$ 20,001-\$ 30,000$ & & & & & & & & -.121 \\
\hline$\$ 30,001-\$ 40,000$ & & & & & & & & -.00352 \\
\hline Base: $\$ 40,001-\$ 50,000$ & & & & & & & & \\
\hline$\$ 50,001-\$ 60,000$ & & & & & & & & .075 \\
\hline$\$ 60,001-\$ 70,000$ & & & & & & & & $.154 *$ \\
\hline$\$ 70,001-\$ 100,000$ & & & & & & & & $.167 *$ \\
\hline More than $\$ 100,000$ & & & & & & & & $.36^{* * *}$ \\
\hline Constant & $4.4 * * *$ & $4.45 * * *$ & $4.47^{* * *}$ & $4.42 * * *$ & $4.29 * * *$ & $4.3 * * *$ & $4.27^{* * *}$ & $4.35^{* * *}$ \\
\hline $\mathrm{N}$ & 5164 & 5164 & 5151 & 5151 & 5148 & 4850 & 4802 & 4121 \\
\hline $\mathrm{r} 2$ & .00395 & .0117 & .0125 & .0264 & .0333 & .0451 & .0511 & .0723 \\
\hline r2_a & .00376 & .0105 & .0112 & .0242 & .0308 & .042 & .0477 & .0664 \\
\hline rmse & .854 & .851 & .85 & .844 & .841 & .831 & .829 & .831 \\
\hline 11 & -6509 & -6489 & -6465 & -6429 & -6405 & -5975 & -5906 & -5072 \\
\hline
\end{tabular}


Table 4.1 and 4.2 provide detail on how the relationship between individual life satisfaction and standard deviation life satisfaction in the ward is impacted by controls. The coefficient -1.255 for Model $1(a=4.842, b=-1.255, S E=0.221, p<0.001)$ in Table 4.1 is very close to the -1.233 from the ecological model in Figure 4.10. However, once I control for the attributes of individuals, the influence of the spread of life satisfaction in the ward diminishes.

Model 2 introduces the city variable, Models 3 to 5 introduce the demographic controls, and Models 6 to 8 introduce the 'achievement' controls. Finally, a number of subjective controls are introduced in Models 9 to 11, although these are not presented in Tables 4.1 and 4.2. Bearing in mind the likely endogenous relationship between SWB and the subjective controls, I will first interpret the impacts of controls up to the introduction of income groups in Model 8, and will consider the influence of Models 9 through 11 separately.

In Table 4.1 (2012), the relationship between standard deviation life satisfaction and individual life satisfaction is statistically significant $(p<0.001)$ across all models. The introduction of a city control in Model 2 decreases the magnitude of the coefficient from -1.255 to -1.048 . Such a decline suggests that there are unobserved features of the cities which raise or lower wellbeing over and above the negative effect of dispersion in wellbeing within the wards of that city. Even in Model 8, Christchurch residents are much less likely to be satisfied with life than Auckland residents, while residents from Wellington, Porirua and Dunedin are all much more likely to be satisfied than Auckland residents.

Introducing sex appears to have almost no impact on the effect of standard deviation within an individual's ward, while introducing age group and ethnicity reduces the effect of life satisfaction inequality slightly to -0.992 by Model 5 . Introducing employment status in Model 6 further reduces the impact of life satisfaction inequality, while introducing education actually increases its effect. Introducing income in Model 8 reduces the coefficient of standard deviation life satisfaction in an individual's ward down to $-0.918(a=4.385, b=-0.918, S E=0.126, p<0.001)$, and the $\mathrm{R}^{2}$ is $0.093 .^{13}$

\footnotetext{
${ }^{13}$ The $\mathrm{R}^{2}$ value will always be lower when analysing a relationship at the individual level when compared to the ecological relationship (as in the earlier scatterplots and regressions) owing to the increased number of observations and associated variance between them.
} 
In summary, the estimates Table 4.1 confirms that part of the negative effect of life satisfaction inequality within city wards is due to the composition of their particular residents. Even so, the reductions are relatively minor, and in all Models from 1 through to 8 the inequality in wellbeing in New Zealand wards continues to have a highly significant negative effect on the wellbeing of the sampled individuals.

In keeping with the results of the ecological regressions in section 4.1, by 2014 the relationship between individual life satisfaction and its standard deviation within wards is lower than in 2012 at -0.704 , but remains statistically significant $(p<0.001)$ in all models. Interestingly, the impact of each control on this relationship is quite different in 2014 compared to 2012. In contrast to 2012, the introduction of the city control in Model 2 of 2014 actually strengthens the negative effect of the coefficient for life satisfaction inequality to -0.812 . Christchurch residents are again much less satisfied than those in other cities throughout the models.

In Table 4.2 the coefficient decreases in magnitude with the introduction of each of the three demographic controls, and also decreases with the introduction of employment status in Model 6. Educational attainment in Model 7 increases the coefficient, while controlling for income group in Model 8 increases the coefficient by a considerable amount up to $-0.900(a=4.352, b=-0.900, S E=0.152, p<0.001)$. The $\mathrm{R}^{2}$ value in Model 8 is 0.072 .

So what does a comparison of the 2012 and 2014 results indicate? The most notable impact of the controls is the convergence of the effect of life satisfaction inequality between 2012 and 2014. While initially the coefficient of the relationship between individual life satisfaction and standard deviation life satisfaction was much greater in 2012 than in 2014, the introduction of controls makes them much more similar. The coefficient falls from -1.255 in Model 1 to -0.918 in Model 8 of 2012, while it rises from -0.704 in Model 1 to -0.900 in Model 8 of 2014. In summary, it appears the standard deviation of life satisfaction in an individual's ward has a negative impact on their life satisfaction, even after controlling for the city of residence and a number of demographic and achievement variables.

The number of observations being drawn on in each model (' $N$ ') decreases slightly in both years with each new model, with a reasonably large drop in Model 6 as employment status is introduced, and a far larger one in Model 8 when income is introduced. The fall in $\mathrm{N}$ is a result of individuals not responding to the question. 
Questions about personal income often have the greatest number of missing responses in surveys, as many individuals tend to view their total income as a private and sensitive topic (Ripahn \& Serfling, 2005). However, research indicates that those who choose not to respond to income and wealth questions in surveys are largely spread across the population, indicating I am unlikely to be missing out on a particular subset of the population as a result (Ripahn \& Serfling, 2005).

As expected, introducing the subjective variables in Models 9 through 11 increases the $\mathrm{R}^{2}$ by a large amount in both 2012 and 2014. In 2012 the $\mathrm{R}^{2}$ increases to 0.350 in Model 11, and in 2014 it increases to 0.335 . Introducing subjective health decreases the coefficient of standard deviation life satisfaction in 2012, and increases it in 2014. Controlling for a sense of community does the opposite, increasing the coefficient in 2012 and decreasing it in 2014. Finally, the introduction of self-rated isolation has the largest effect of all the controls, reducing the magnitude of standard deviation life satisfaction in both years by a considerable amount.

The coefficients of the standard deviation of life satisfaction for 2012 and 2014 become more similar in Model 11 than in Model 8, with a coefficient of -0.699 in 2012 $(a=3.858, b=-0.699, S E=0.143, p<0.001)$, and of -0.696 in $2014(a=3.835, b=-0.696$, $S E=0.157, p<0.001)$. Once I introduce geographic, demographic, achievement and subjective controls, a very similar negative relationship between individual life satisfaction and the inequality in life satisfaction in a ward is visible in both 2012 and 2014.

Figure 4.14 and 4.15 visualise the tabular information in Model 8 of Table 4.1 and 4.2 respectively ${ }^{14,15}$. They exclude Models 9 through 11 in order to explicitly focus on the effect of common correlates of SWB in the literature: the ascribed and achieved control variables specified in Models 3 to 8 , as well as the regional controls of Model 2. The red line represents 0 on the $x$ axis, and indicates when the variable has no significant effect. Each plotted point represents the effect of the variable on life satisfaction (on a 5-point scale), and the horizontal lines around these points represent $95 \%$ confidence intervals.

\footnotetext{
${ }^{14}$ The coefficient plots in Figures 4.18 and 4.19 are also cluster-controlled, increasing the accuracy of the standard errors.

${ }^{15}$ For more information on these coefficient plots and how they are formed, see Jann (2014) for a discussion from the creator.
} 
Figure 4.18: Coefficient plot of standard deviation life satisfaction and controls on individual life satisfaction in 2012

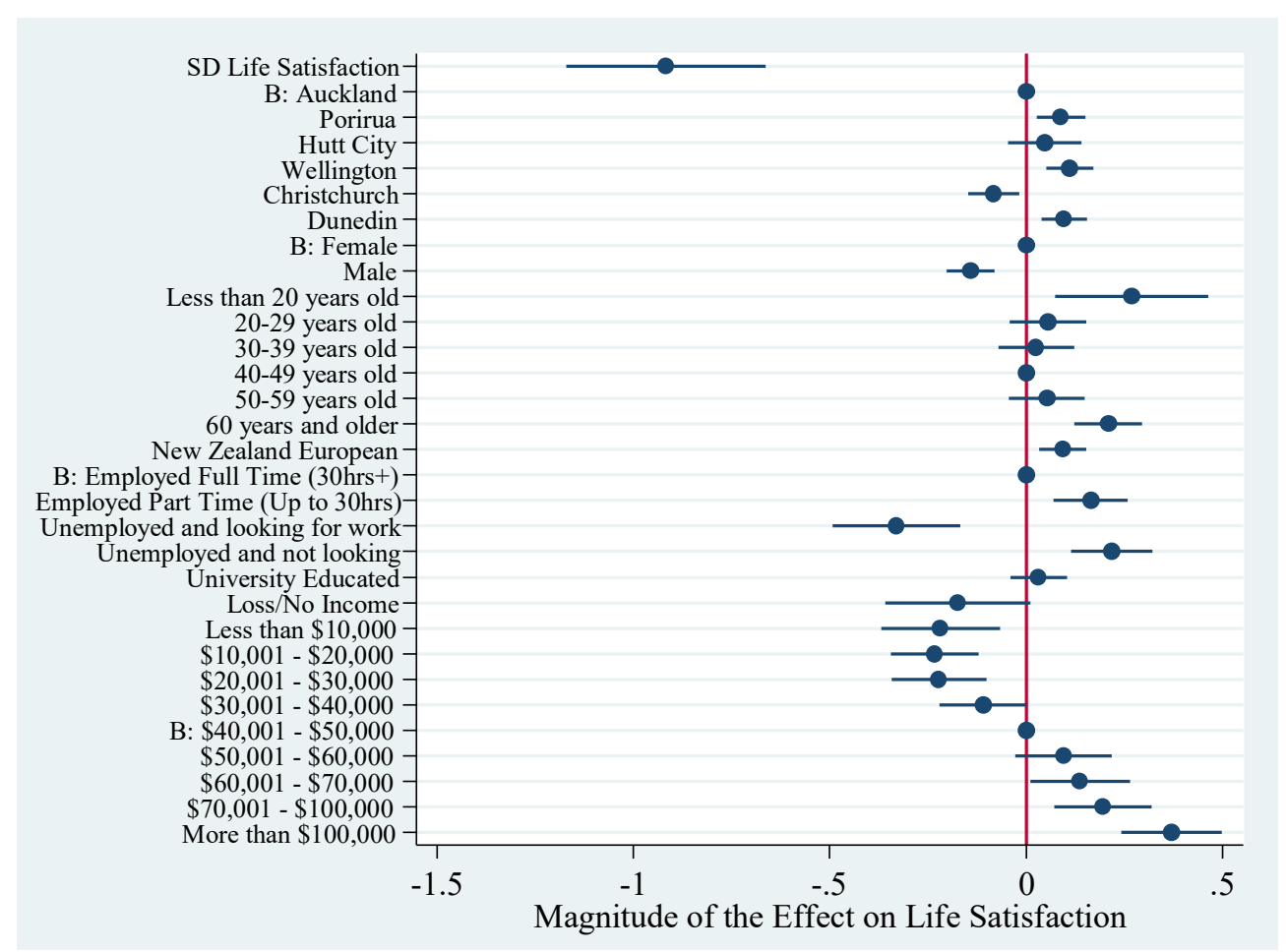

Figure 4.18: QoLS, 2012, New Zealand

Figure 4.19: Coefficient plot of standard deviation life satisfaction and controls on individual life satisfaction in 2014

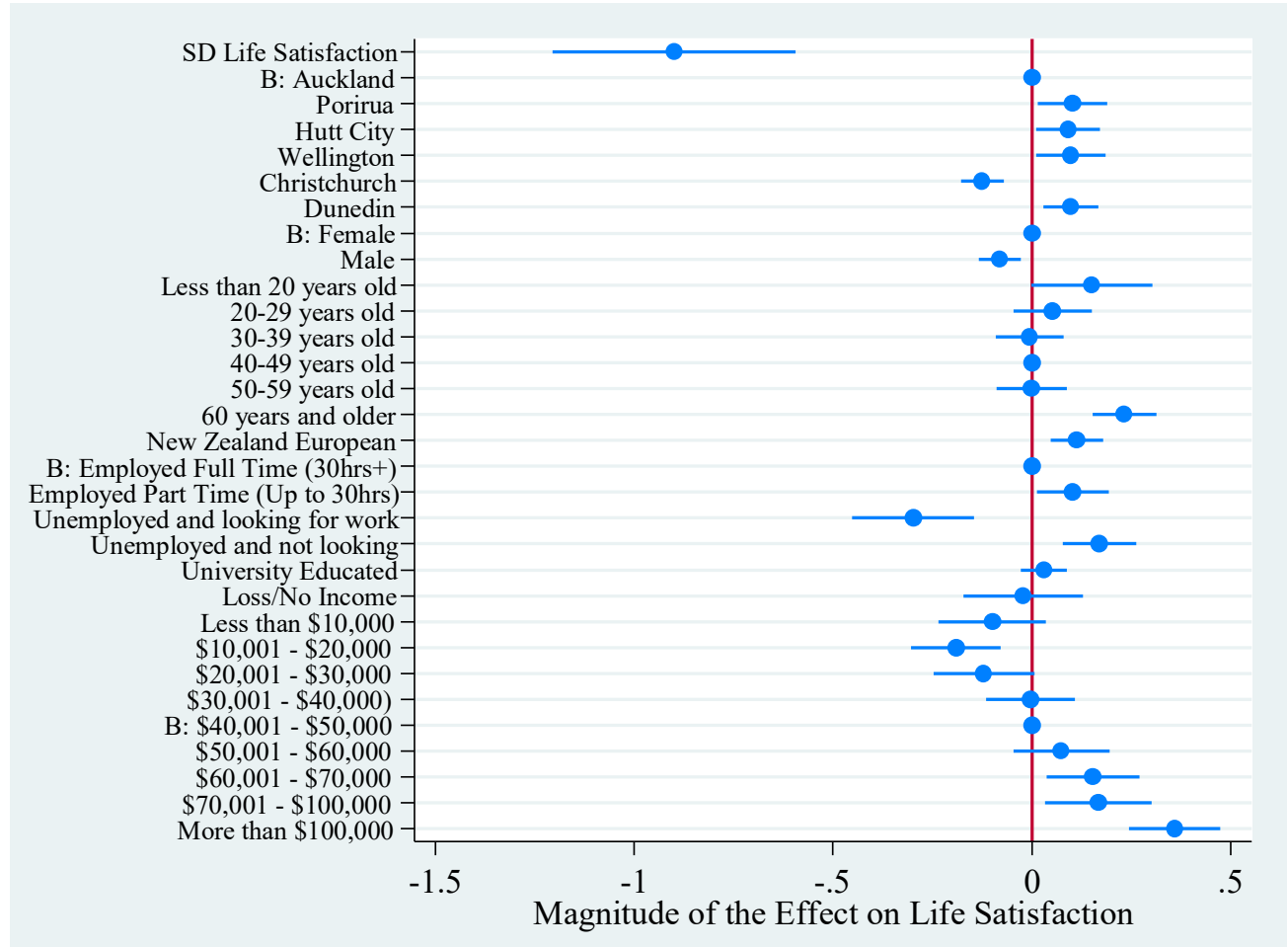

Figure 4.19: QoLS, 2014, New Zealand 
While Figures 4.18 and 4.19 are useful for visualising the impact of controls on wellbeing, perhaps their greatest use is visualising the impact of standard deviation life satisfaction relative to the controls. Of the controls added to the SWB and SWB relationship, being 'unemployed and looking for work' has the greatest impact on life satisfaction in both 2012 and 2014: a common finding in the literature (Dolan et al., 2008; Helliwell, 2003; Layard et al., 2012; Winkelmann, 2009). However, in both years it is clear that a theoretical one unit increase in standard deviation life satisfaction has a greater negative effect on an individual's life satisfaction than unemployment ${ }^{16}$. Such a result suggests the inequality in wellbeing around an individual has a large influence on their wellbeing, and should be given greater attention in future research and policy.

The results for The Primary Hypothesis indicate that SWB is negatively related to SWB inequality at the sub-national level. While these results are always significant at the ecological level when using the 2012 QoLS, they are only significant for the 2014 ecological regressions when examining Auckland wards or New Zealand wards overall. Examining the relationship between life satisfaction and life satisfaction inequality at the sub-national level reveals that controls are responsible for a large part of this difference. After controlling for a comprehensive list of variables known to influence life satisfaction, I find a similar negative relationship between an individual's life satisfaction and the inequality in life satisfaction in their ward in both 2012 and 2014.

The Parochialism Hypothesis that wellbeing is most sensitive to wellbeing inequality at smaller scales is largely not supported using the QoLS data. In the ecological regression in 2012, the effect of wellbeing inequality is greater at the city level than at the ward level. In 2014, the city level effect of inequality is non-significant, whereas some of the ward level effects are significant. While the 2014 data does appear to support The Parochialism Hypothesis to some degree, more concrete evidence is needed $^{17}$.

\footnotetext{
${ }^{16}$ It is worth noting that the range of standard deviation does not appear to differ by as much as one unit when using this QoLS dataset, with the maximum range being closer to 0.5 units. As such, discussing a 'one unit' increase in standard deviation life satisfaction is largely hypothetical within this thesis, and is used for clarity of communication.

${ }^{17}$ The Parochialism Hypothesis will be discussed again in later chapters, but the QoLS is the most appropriate for testing the hypothesis due to its distinct geographic variables. In this thesis I have dealt with one kind of group (the geographic kind) to test parochialism. It is likely that sensitivity to SWB inequality will vary with membership to other successively smaller groups as well (e.g. sub-groups of an ethnicity relative to the ethnicity, particular workplaces relative to an industry, or members of a particular religious denomination relative to the broader faith). Future research can explore these possibilities and can retest The Parochialism Hypothesis using a richer sample of cities.
} 


\section{3: Summary}

The primary aim of this chapter has been to use the QoLS to assess whether those living within a country are sensitive to the inequality of wellbeing in their local area. Taken together, the results support The Primary Hypothesis. At the ecological level, the negative relationship between mean and standard deviation life satisfaction was significant both for cities and wards.

The individual level regressions allowed for the relationship between wellbeing and wellbeing inequality to include controls for individual attributes. Introducing demographic and achievement based controls did not diminish the statistical significance of the effect of wellbeing inequality on individual wellbeing. While controls did reduce the slope of the relationship in 2012 , they actually made the slope steeper in 2014.

In terms of magnitude, the effect of a theoretical one unit increase in standard deviation life satisfaction in an individual's ward had a larger negative effect on life satisfaction than being unemployed - one of the major attributes associated with lower life satisfaction. The statistical significance of SWB inequality persisted even after introducing subjective measures of health and social capital, both strong correlates of SWB. All regressions were adjusted for the clustering of individuals by wards.

Geographic groups were also used to examine the difference in sensitivity to SWB inequality. I expected inequality in smaller groups like wards would have had a larger impact on life satisfaction than inequality in cities (The Parochialism Hypothesis). However, in 2012 the negative effect of SWB inequality on mean SWB was stronger at the city level than at the ward level. The opposite is true for 2014, indicating inconsistent results by year. The potential reasons underlying this lack of support for The Parochialism Hypothesis will be discussed further in the conclusion chapter.

This chapter provides comprehensive evidence of a negative relationship between wellbeing and wellbeing inequality at both the ecological and individual level within New Zealand. However, at present it is still unclear what mechanisms drive this sensitivity to SWB inequality. The following chapter aims to provide initial answers to the question - what makes people sensitive to local wellbeing inequality? 


\section{Chapter 5: What Mechanisms Drive Sensitivity to Wellbeing Inequality?}

Previously I demonstrated that a negative relationship exists between wellbeing and local wellbeing inequality across urban New Zealand. In this chapter I examine the extent to which this relationship is driven by an individual's perceptions of fairness in society and their altruistic tendencies. To do so I draw on the 2008, 2010, and 2012 waves of the New Zealand General Social Survey (NZGSS). The NZGSS is conducted biennially by Statistics New Zealand with the aim of determining how well people are faring throughout the country. ${ }^{18}$

I begin with testing my primary hypothesis on the three survey years available by re-estimating the negative relationship between life satisfaction and life satisfaction inequality. The remainder of the chapter will focus on the analysis of The Fairness Hypothesis and The Altruism Hypothesis as stated in Chapter 2.

\section{1: The Primary Hypothesis - Confirming Wellbeing is Sensitive to Local Wellbeing Inequality}

My primary hypothesis is that wellbeing inequality negatively impacts both average and individual wellbeing, as in Chapter 4. I begin with the ecological relationship between mean life satisfaction and standard deviation life satisfaction by region of New Zealand for each year.

But first, an introduction to the life satisfaction measure is necessary. Like in the QoLS, the NZGSS uses a 5-point Likert scale for life satisfaction. Respondents were asked “...how do you feel about your life as a whole right now?” and were given options ranging from 1 'very dissatisfied' to 5 'very satisfied'. In 2008 the mean life satisfaction score across the dataset was 4.067, in 2010 it was 4.086, and in 2012 it was 4.075 (see Figure 3.5 for a histogram of life satisfaction by year).

For this analysis I draw on the only version of the NZGSS available to researchers outside of Statistics New Zealand: the confidentialised unit record files (CURFs). Many of the variables in the CURFs are collapsed into a smaller number of

\footnotetext{
${ }^{18}$ See Appendix A for technical details on the NZGSS including data collection, the variables available, and the manipulation of those variables for this research.
} 
categories in order to protect the confidentiality of the respondent. This does limit the level of locational detail I have available in the 'region' variable.

However, data is also available on the urbanity of the respondent's location, and I have used this to create a combined urban-region variable. The new variable contains 24 distinct geographic areas consisting of four urban-rural identifiers within each of the six regions, as shown in Table 5.1. To allow for sufficient variation in life satisfaction, any group with 30 or fewer responses in any one year was excluded from the analysis for only that year. The groups not included for that reason are: Secondary Urban Auckland (2012), Minor Urban Auckland (2008, 2010), Rural Auckland (2012), Minor Urban Wellington $(2008,2010)$ and Rural Wellington $(2008,2010,2012)$. After these observations are excluded, 21 spatial groups still remain in each of 2008, 2010, and 2012. The standard deviation of life satisfaction was generated for each of these groups. $^{19}$

Table 5.1: Details on the NZGSS urban-region variable

Label
\begin{tabular}{|c|l|c|c|l|c|}
\hline A1 & Main Urban Auckland & 5,505 & A3 & Minor Urban Auckland & 67 \\
\hline W1 & Main Urban Wellington & 3,237 & W3 & Minor Urban Wellington & 80 \\
\hline N1 & Main Urban Northland Grp & 2,590 & N3 & Minor Urban Northland Grp & 304 \\
\hline RN1 & Main Urban Rest NI & 2,981 & RN3 & Minor Urban Rest NI & 754 \\
\hline C1 & Main Urban Canterbury & 2,562 & C3 & Minor Urban Canterbury & 378 \\
\hline RS1 & Main Urban Rest SI & 1,921 & RS3 & Minor Urban Rest SI & 699 \\
\hline A2 & Secondary Urban Auckland & 112 & A4 & Rural Auckland & 124 \\
\hline W2 & Secondary Urban Wellington & 210 & W4 & Rural Wellington & 77 \\
\hline N2 & Secondary Urban Northland Grp & 154 & N4 & Rural Northland Grp & 707 \\
\hline RN2 & Secondary Urban Rest NI & 757 & RN4 & Rural Rest NI & 541 \\
\hline C2 & Secondary Urban Canterbury & 603 & C4 & Rural Canterbury & 315 \\
\hline RS2 & Secondary Urban Rest SI & 495 & RS4 & Rural Rest SI & 495 \\
\hline \hline
\end{tabular}

Figure 5.1 displays a scatterplot of mean life satisfaction against standard deviation life satisfaction across New Zealand regions for the years 2008, 2010, and 2012. The first year, 2008, is represented by the darkest shade of green, with individual

\footnotetext{
${ }^{19}$ The distribution of standard deviation life satisfaction was tested for outliers in each of the three years. None were found.
} 
$68 \mid \mathrm{P}$ a g e

groups in the shape of hollow squares and a long-dash style line of best fit. 2010 is represented by a medium shade of green, with groups represented by triangles and a short-dash style line of best fit. 2012 is the lightest shade of green, with circular points and a solid line of best fit.

An initial glance seems to support the negative relationship detailed in Chapter 5. The mean life satisfaction of the region appears to fall with the standard deviation of life satisfaction across New Zealand regions in each of the three survey years. The relationship appears to be weakest in 2008 (note the shallow slope and wide confidence intervals) and strongest in 2012. OLS regressions of the lines displayed in Figure 5.1 give a coefficient of -0.315 for $2008,-0.627$ for 2010 , and -0.699 for 2012 . However, with $p$-values of $0.471,0.129$ and 0.141 respectively, none of these regression slopes are statistically significant. These 'regions' in the NZGSS are much larger than the cities used in the QoLS, indicating that a more local geographic variable may be necessary to more fully test the relationship between SWB and SWB inequality.

Figure 5.1: The relationship between mean and standard deviation life satisfaction by region

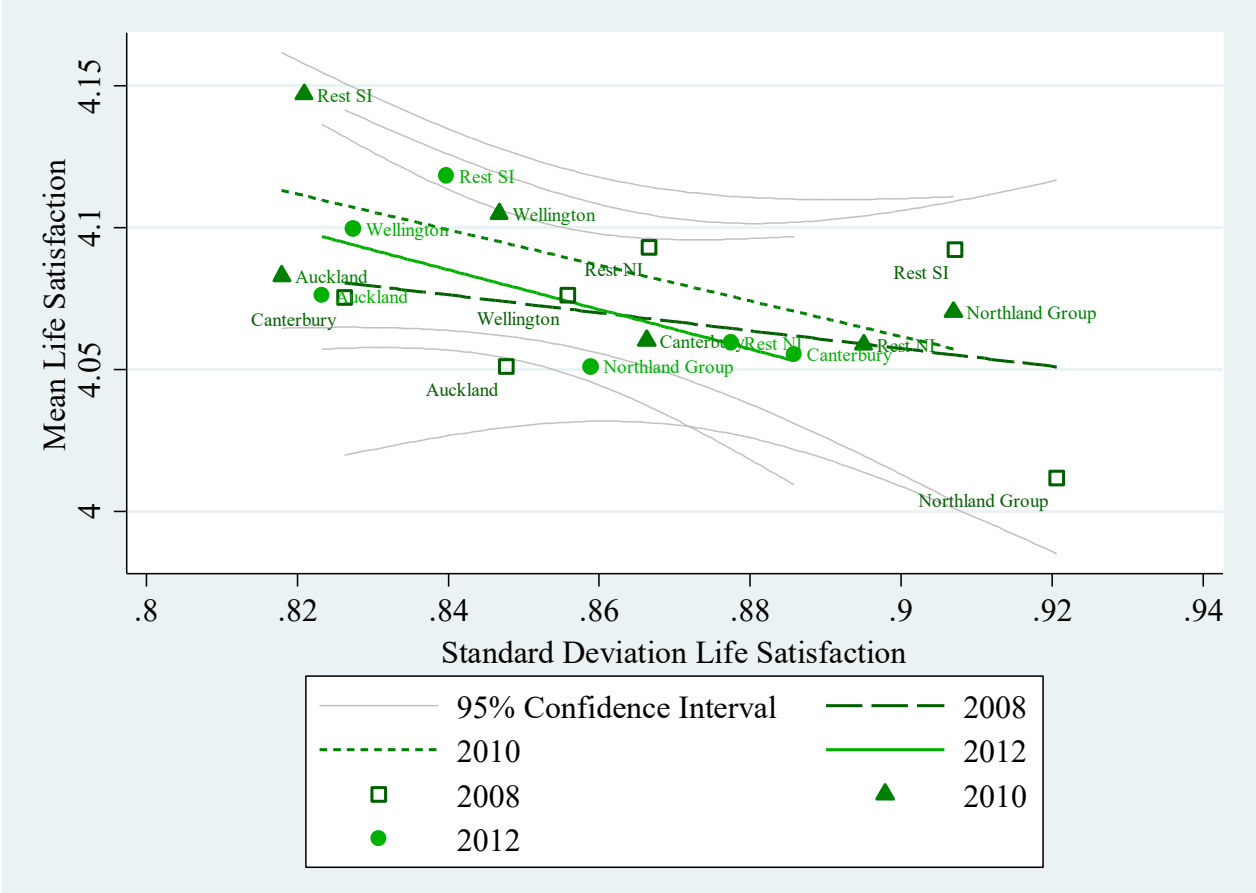

Figure 5.1: NZGSS, 2008, 2010, and 2012, New Zealand

In Figure 5.2 I have used the new, more comprehensive geographic variable to create a scatterplot of mean life satisfaction against standard deviation life satisfaction for each of the three years. In all three years an apparent negative relationship between 
69|P a g e

mean life satisfaction and life satisfaction inequality in an area is evident. While the relationship appears comparable in magnitude in 2010 and 2012, it again seems to be weaker in 2008.

The regressions for Figure 5.2 are more promising than for the previous figure. The magnitude of the coefficient is at its lowest in 2008, with a single unit increase in standard deviation life satisfaction associated with a reduction in mean life satisfaction of only $-0.647(a=4.644, b=-0.647, S E=0.277, p<0.05)$, and an $\mathrm{R}^{2}$ of 0.224 . The effect in 2010 is at the strongest of the three years, both statistically and substantively $(a=4.928, b=-0.951, S E=0.269, p<0.01)$, and the $\mathrm{R}^{2}$ value in this year is 0.397 , indicating that standard deviation of life satisfaction explains more of the variance in mean life satisfaction in this year than in others. The coefficient in 2012 is -0.760 $(a=4.714, b=-0.760, S E=0.313, p<0.05)$, and the $\mathrm{R}^{2}$ is 0.237 .

\section{Figure 5.2: The relationship between mean and standard deviation life satisfaction by urban-region}

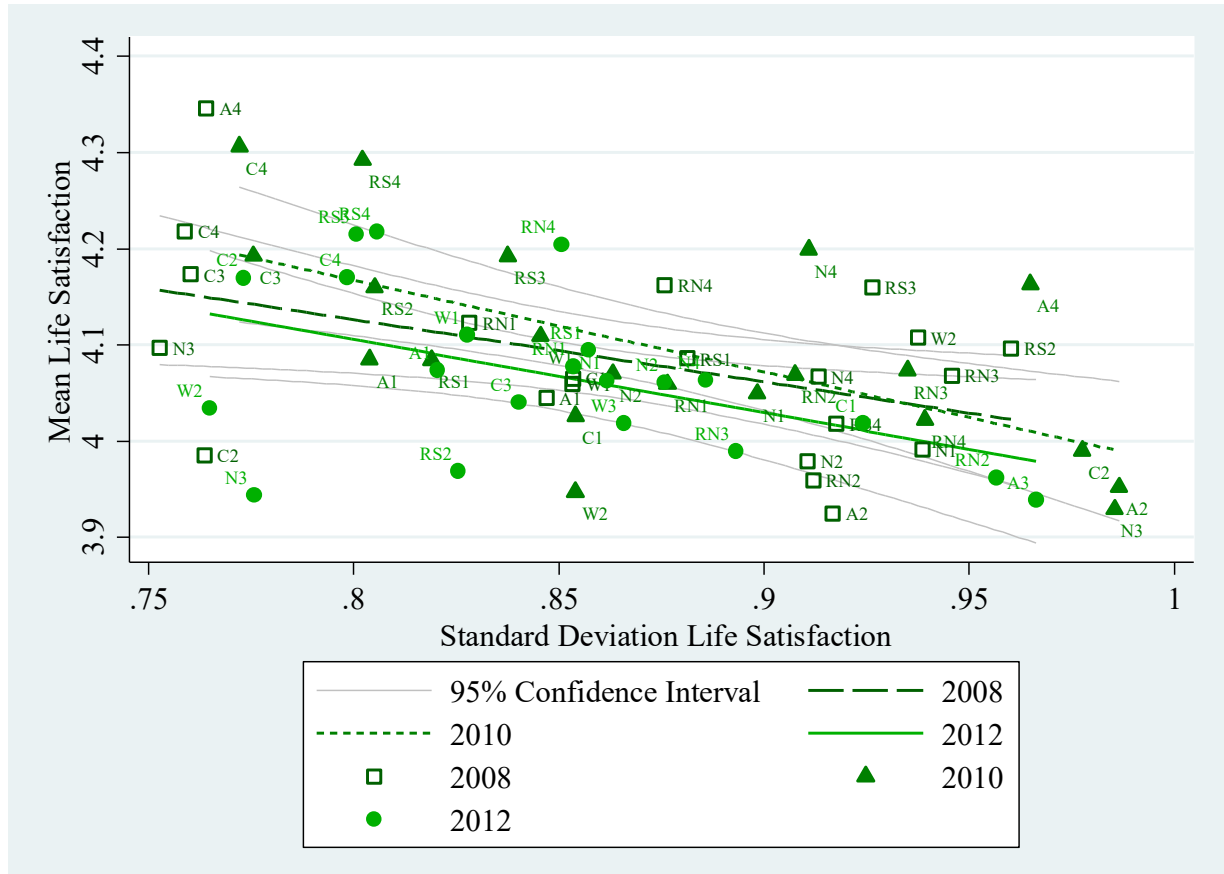

Figure 5.2: NZGSS, 2008, 2010, and 2012, New Zealand

Why might sensitivity be greater in 2010 than in other years? 2010 followed two years of a worsening economy, with a stark rise in the unemployment rate in contrast to 2008 (Morrison, 2015a; also see Table 3.2). Groups may have been more sensitive to the inequality around them as a result of increasingly obvious disparities. While I do explore the different macro conditions prevailing in the years of these 
70|P a g e

surveys to some extent, a more comprehensive coverage of changes of macro conditions over time lies outside the scope of this thesis.

Figure 5.3 and the associated regressions ${ }^{20}$ indicate that The Primary Hypothesis is supported in the NZGSS in 2008, 2010, and 2012 at the ecological level. Combined with the findings in Chapter 4 using the QoLS, these results are further confirmation that mean SWB is negatively related to SWB inequality at the sub-national level.

However, the individual level relationship may differ from the ecological relationship (Robinson, 1950). I therefore ran a series of 13 regression models of individual life satisfaction against standard deviation life satisfaction, introducing a new control in each model. Following a similar sequence to Chapter 4, I introduce the geographic control first (region), followed by demographic controls (sex, age group, New Zealand European, and Māori), and then achievement controls (employment status, university education, and finally income in Model 8).

Two suitable control variables are available in the NZGSS that are not in the QoLS. I include these after the achievement controls, but before the subjective controls that correlate highly with SWB. The first new control I introduce is the Economic Living Standards Index (ELSI) in Model 9. An individual's ELSI score is composed of respondents answers to a number of questions about what they can and cannot afford to do on a regular basis, as well as the things that they have access to within their homes (e.g. "do you have a night out at least once a fortnight?" or "do you have a washing machine?"). The ELSI score ranges from low (0) to high (31). It is worth noting that ELSI also has a few subjective questions within it (e.g. satisfaction with standard of living) which may inflate its correlation with life satisfaction. ${ }^{21}$ Nonetheless, ELSI represents a more comprehensive measure of economic welfare than just income, and so it is a useful control to include.

The second new control I introduce is a binary partnership status variable in Model 10. Research consistently indicates that people who are married or partnered score higher on SWB measures than those who are not partnered, and it has become a common control to include when available (Dolan et al., 2008; Helliwell, 2003; Layard et al., 2012).

\footnotetext{
${ }^{20}$ Residual plots of the geographic groups for each regression indicate they are randomly distributed.

${ }^{21}$ For more information on ELSI, visit www.msd.govt.nz/about-msd-and-our-work/publicationsresources/monitoring/living-standards/index.html
} 
Model 11 introduces subjective health, the first directly subjective control. Model 12 introduces a binary subjective variable, asking whether respondents' feel a sense of belonging in New Zealand. Model 13 introduces a variable asking about the degree of social isolation that the individual feels.

As displaying the models for all three years would take a great deal of space, only the 2012 estimates table is presented on the following page. The tables for 2008 and 2010 will be discussed in the text, but will not be presented. Table 5.2 displays estimates of Models 1 to 10 for the 2012 data of the NZGSS (the only year common to the NZGSS and the QoLS). As in Chapter 4, the subjective variables in the later models have been excluded from the display, but were still analysed and will briefly be discussed.

The most important aspect of this estimates tables for my thesis is the effect that standard deviation life satisfaction in an individual's area has upon their life satisfaction. The results for the NZGSS are similar to those in the QoLS. In 2012, life satisfaction inequality has a strong and significant effect on individual life satisfaction in Model 1 $(a=4.770, b=-0.819, S E=0.205, p<0.001)$. The statistical significance grows weaker with the introduction of regional controls in Model 2, although the coefficient size increases slightly. The introduction of demographic controls decreases the coefficient again, as does controlling for employment status. However, introducing university education raises the coefficient, and introducing income raises the coefficient and the significance. In Model 8 of Table 5.2, the coefficient of life satisfaction inequality on individual life satisfaction is $-0.787(a=4.484, b=-0.787, S E=0.201, p<0.001)$, quite similar to that in Model 8 of Table $4.1(b=-0.918)$ in spite of the differences in data collection methods and variable construction between the QoLS and the NZGSS. The estimates for 2012 in the NZGSS are consistently lower in magnitude than in the QoLS, although this difference does decrease with the addition of each new model.

The effect size drops slightly with the introduction of ELSI and partnership status in Models 9 and 10, falling to $-0.664(p<0.001)$ in Model 10. After all subjective controls are introduced in Model 13, the effect of standard deviation life satisfaction is still negative, but its magnitude is reduced ( $a=2.847, b=-0.308, S E=0.145, p<0.05)$. 
Table 5.2: Models of individual life satisfaction against standard deviation life satisfaction of urban-region group, 2012 (cluster-controlled)

\begin{tabular}{|c|c|c|c|c|c|c|c|c|c|c|}
\hline & Model 1 & Model 2 & Model 3 & Model 4 & Model 5 & Model 6 & Model 7 & Model 8 & Model 9 & Model 10 \\
\hline SD Life Satisfaction & $-.819 * * *$ & $-.908 * *$ & $-.901 * *$ & $-.839 * *$ & $-.763 * *$ & $-.637 * *$ & $-.745 * *$ & $-.787 * * *$ & $-.751 * * *$ & $-.664 * * *$ \\
\hline \multirow{2}{*}{\multicolumn{11}{|c|}{ Region (Base Auckland) }} \\
\hline & & & & & & & & & & \\
\hline Wellington & & .0263 & .0261 & .0192 & .0043 & -.00317 & -.00071 & .00384 & .00676 & .00371 \\
\hline Northland Group & & .00856 & .00768 & -.00907 & -.00567 & -.0184 & .0082 & .0346 & .0235 & .0113 \\
\hline Rest of North Island & & .0317 & .031 & .0193 & .00939 & .00494 & .0316 & $.0573 *$ & $.0396^{*}$ & .0289 \\
\hline Canterbury & & .0344 & .0338 & .0184 & -.0187 & -.028 & .0007 & .0249 & -.0182 & $-.0294 * *$ \\
\hline Rest of South Island & & $.0561 *$ & $.0556^{*}$ & .041 & .0067 & -.00426 & .0192 & $.0509 *$ & .0184 & .00838 \\
\hline \multicolumn{11}{|l|}{ Sex (Base Female) } \\
\hline Male & & & -.0316 & -.0291 & -.0314 & $-.0636 * *$ & $-.0548 * *$ & $-.108 * * *$ & $-.103 * * *$ & $-.122 * * *$ \\
\hline \multicolumn{11}{|l|}{ Age Group, Decades } \\
\hline $15-19$ & & & & $.124 * * *$ & $.14 * * *$ & $.259 * * *$ & $.305 * * *$ & $.273 * * *$ & .0929 & $.19 * *$ \\
\hline $20-29$ & & & & $.101 * * *$ & $.114 * * *$ & $.144 * * *$ & $.152 * * *$ & $.206 * * *$ & $.154 * * *$ & $.182 * * *$ \\
\hline $30-39$ & & & & .0603 & $.0699 *$ & $.0746 * *$ & $.0634 *$ & $.0732 * *$ & $.0799 *$ & $.0711 *$ \\
\hline \multicolumn{11}{|l|}{ Base: 40-49 } \\
\hline $50-59$ & & & & .00821 & .00012 & .00093 & .011 & .0217 & -.0593 & -.0531 \\
\hline $60-69$ & & & & $.191 * * *$ & $.174 * * *$ & $.233 * * *$ & $.255 * * *$ & $.302 * * *$ & $.0627^{*}$ & $.0671 *$ \\
\hline $70-79$ & & & & $.253 * * *$ & $.229 * * *$ & $.399 * * *$ & $.426 * * *$ & $.516 * * *$ & $.101 * *$ & $.106 * *$ \\
\hline $80+$ & & & & $.182 * * *$ & $.15 * * *$ & $.349 * * *$ & $.374 * * *$ & $.483 * * *$ & -.0133 & .0331 \\
\hline Māori & & & & & $-123 * * *$ & $-0905 * *$ & $-0571 *$ & - 0447 & 00524 & 0157 \\
\hline New Zealand European & & & & & $.111 * *$ & $.0849 *$ & $.101 * *$ & $.0658 *$ & .00395 & .00764 \\
\hline & & & & & & & & & & \\
\hline \multicolumn{11}{|l|}{$\begin{array}{r}\text { Employment Status } \\
\text { (Base Employed) }\end{array}$} \\
\hline Unemployed & & & & & & $-.437 * * *$ & $-.418 * * *$ & $-.319 * * *$ & $-.124 *$ & -.0887 \\
\hline Not in Labour Force & & & & & & $-.261 * * *$ & $-.246 * * *$ & $-.149 * * *$ & -.0238 & .00449 \\
\hline
\end{tabular}




\begin{tabular}{|c|c|c|c|c|c|c|c|c|c|c|}
\hline \multirow[b]{3}{*}{ University Educated } & \\
\hline & & & & & & & & & & \\
\hline & & & & & & & $.195 * * *$ & $.132 * * *$ & .0473 & .0391 \\
\hline \multirow{2}{*}{\multicolumn{11}{|c|}{ Income }} \\
\hline & & & & & & & & & & \\
\hline Loss/Zero & & & & & & & & $.163 * *$ & .0411 & -.0307 \\
\hline$\$ 1-\$ 5,000$ & & & & & & & & $.18^{* *}$ & .0386 & -.00641 \\
\hline$\$ 5,001-\$ 10,000$ & & & & & & & & $.132 *$ & $.122 *$ & .0878 \\
\hline$\$ 10,001-\$ 15,000$ & & & & & & & & $-.12 *$ & -.0129 & -.0347 \\
\hline$\$ 15,001-\$ 20,000$ & & & & & & & & -.0732 & -.0161 & -.0133 \\
\hline$\$ 20,001-\$ 25,000$ & & & & & & & & -.0947 & -.0358 & -.0283 \\
\hline \multicolumn{11}{|l|}{ Base: $\$ 25,001-\$ 30,000$} \\
\hline$\$ 30,001-\$ 35,000$ & & & & & & & & .0463 & .0324 & .0241 \\
\hline$\$ 35,001-\$ 40,000$ & & & & & & & & .0927 & -.00588 & -.00741 \\
\hline$\$ 40,001-\$ 70,000$ & & & & & & & & $.219 * *$ & .0451 & .034 \\
\hline$\$ 70,001-\$ 100,000$ & & & & & & & & $.293 * * *$ & .0299 & .0156 \\
\hline$\$ 100,00-\$ 150,000$ & & & & & & & & $.345^{* *}$ & .0199 & .0106 \\
\hline$\$ 150,001$ and above & & & & & & & & $.482 * * *$ & .0953 & .0846 \\
\hline \multirow{2}{*}{ ELSI } & & & & & & & & & & \\
\hline & & & & & & & & & $.05 / 2$ an & $.0552^{2+a n}$ \\
\hline Partnered & & & & & & & & & & $.189 * * *$ \\
\hline Constant & $4.77 * * *$ & $4.82 * * *$ & $4.83 * * *$ & $4.69 * * *$ & $4.58 * * *$ & $4.57 * * *$ & $4.56^{* * *}$ & $4.48^{* * *}$ & $3.38 * * *$ & $3.25 * * *$ \\
\hline $\mathrm{N}$ & 8389 & 8389 & 8389 & 8389 & 8369 & 8364 & 8364 & 8364 & 8089 & 8089 \\
\hline $\mathrm{r} 2$ & .00167 & .00212 & .00246 & .0128 & .0197 & .0403 & .0481 & .0709 & .206 & .216 \\
\hline $\mathrm{r} 2 \mathrm{a}$ & .00155 & .00141 & .00163 & .0112 & .0178 & .0382 & .0459 & .0674 & .203 & .213 \\
\hline rmse & .851 & .851 & .851 & .847 & .844 & .835 & .832 & .822 & .764 & .759 \\
\hline 11 & -10547 & -10546 & -10544 & -10500 & -10446 & -10350 & -10316 & -10215 & -9284 & -9232 \\
\hline
\end{tabular}


In 2010 the data tell a different story. The relationship between individual life satisfaction and local life satisfaction inequality is negative and statistically significant in each of the first 8 models. Without controls, the relationship is weaker than in 2012, with a coefficient of $-0.615(a=4.611, b=-0.615, S E=0.229, p<0.05)$. The coefficient rises in Models 2, 3 and 4, but falls with the introduction of ethnicity in Model 5. The size of the coefficient falls from Model 6 to Model 8, although it retains statistical significance $(a=4.496, b=0.630, S E=0.289, p<0.05)$. The effect of standard deviation life satisfaction on individual life satisfaction becomes non-significant ( $p=0.097)$ with the introduction of ELSI. This variable representing economic welfare likely accounts for a larger portion of the variance in life satisfaction in 2010 owing to the ongoing effects of the period of rapid macroeconomic change over the previous two years. While the relationship remains negative for the rest of the models, statistical significance does not return.

The story is different yet again for the 2008 data. In 2008, Model 1 is not statistically significant $(p=0.970)$. The relationship between life satisfaction and standard deviation life satisfaction becomes significant in Model 2 with the introduction of regional controls $(a=4.582, b=-0.628, S E=0.252, p<0.05)$, and still retains significance in Model 5 after demographic controls are introduced $(a=4.456, b=-0.611$, $S E=0.271, p<0.05)$. However, the coefficient becomes non-significant in Model 6, and significance does not return in any future models.

What might underlie this variation in the relationship between life satisfaction and life satisfaction inequality between the years? While macroeconomic conditions are influential, other possible explanations are institutional. For example, the political climate of 2008. At the time, the Labour Party (a centre-left party) were replaced by the National Party (a centre-right party). It is possible a period of addressing inequality concerns under Labour led to a perception that society was 'equal enough', encouraging people to become less sensitive to the distribution of wellbeing around them, therefore explaining the subsequent switch to National. If this speculation is correct, I can expect to see a difference in the results of The Fairness Hypothesis by year: in 2008, individuals who perceive society to be fairer should be much less sensitive to inequality than individuals who are not, but I will hold off on further discussion of the influence of the political climate until I analyse The Fairness Hypothesis in section 5.2. 
As implied above, the marked increase in the unemployment rate from 2008 to 2010 may have led to individuals being more concerned with their own needs and wellbeing rather than the wellbeing of others. The immediate effects of the 2008 global financial crisis could explain why we see an increase in sensitivity to life satisfaction inequality in 2012 compared with years closer to the crisis.

The final step in assessing The Primary Hypothesis is contrasting the impact of standard deviation life satisfaction on individual life satisfaction relative to the controls. Figure 5.3 displays a plot of the coefficients in Model 10 of Table 5.2. As in the QoLS, it is clear that standard deviation life satisfaction in an area has a stronger negative effect on individual life satisfaction than a number of controls known to impact SWB.

\section{Figure 5.3: Coefficient plot of standard deviation life satisfaction and controls on} individual life satisfaction in 2012

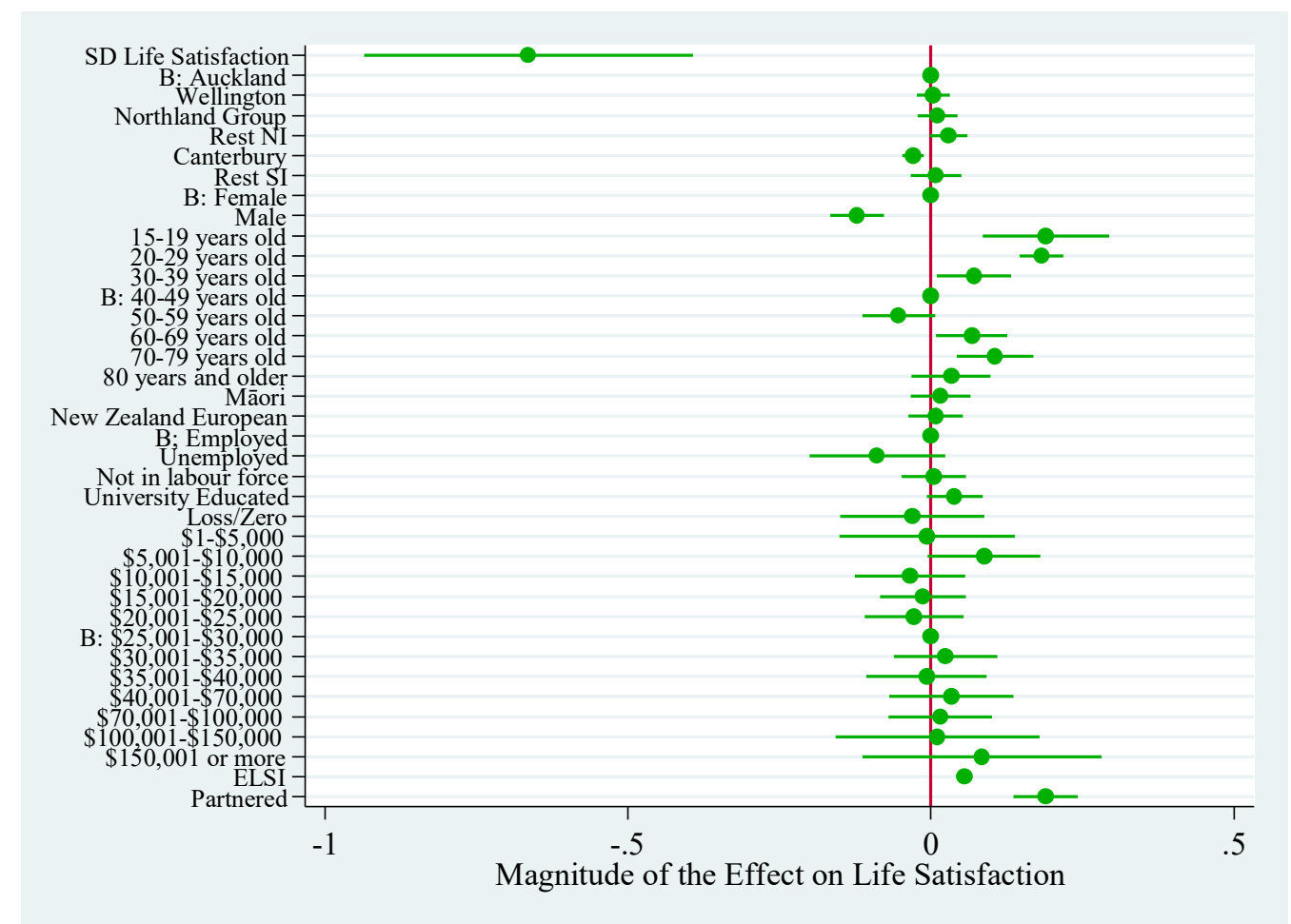

Figure 5.3: NZGSS, 2012, New Zealand

In the previous chapter, I noted that The Parochialism Hypothesis (that sensitivity to wellbeing inequality is greater at smaller scales) was not supported. The QoLS is the most suitable for testing this hypothesis, as it has distinct geographic units nested within each other (i.e. wards within cities). If we set aside the less suitable geographic indicators in the NZGSS, then The Parochialism Hypothesis appears to be supported. The region level regressions were non-significant, while those at a more 
76 | P a g e

local level revealed a significant relationship. I return to The Parochialism Hypothesis in the conclusion.

This section indicates that a negative relationship exists between life satisfaction and sub-national life satisfaction inequality at the ecological level. The relationship between life satisfaction and life satisfaction inequality is less clear at the individual level over time, potentially because sensitivity to life satisfaction inequality may change with the macro conditions in the economy. ${ }^{22}$ Given that SWB inequality has been found to vary over time within the United States (Dutta \& Foster, 2013; Stevenson \& Wolfers, 2008; Veenhoven, 2005), that the same is occurring within New Zealand is certainly within the realm of possibility. However, exploring the mechanisms underlying sensitivity to SWB inequality may shed some light on the differences between years. The following sections will address The Fairness Hypothesis and The Altruism Hypothesis respectively.

\section{2: The Fairness Hypothesis}

The Fairness Hypothesis predicts that individuals who believe society treats people fairly will be less sensitive to wellbeing inequality than those who believe society is less fair. While fairness perceptions have been used to explain the relationship between SWB and income inequality (Katic \& Ingram, 2017; Oishi et al., 2011), they have not yet been tested with regards to wellbeing inequality. Although Goff and colleagues (2016) do contrast people based on beliefs that income inequality is too high (finding that those who believe inequality is too high are more sensitive to wellbeing inequality), such a measure is different from beliefs that people are treated fairly in general. While the former focuses specifically on the fairness of the income generation process, the latter concerns the fairness of interactions between people in society more holistically.

Katic \& Ingram (2017) test whether the life satisfaction scores of those who believe the income generation process is unfair are more negatively impacted by income inequality. They find a significant negative effect, meaning that in high income inequality areas, those who perceive the income generation process to be unfair report a lower life satisfaction.

\footnotetext{
${ }^{22}$ Without longitudinal data, it is difficult to tell whether sensitivity to SWB inequality actually varies over time, or whether variations are due to the different compositions of individuals in the data. The required modelling of repeated cross-sections lies outside of the focus of this thesis.
} 
However, I am not aware of any questions in surveys that ask whether individuals believe the wellbeing generation process is fair, and there are no questions about the income generation process in the NZGSS. As such, how do I approach testing The Fairness Hypothesis as it relates to SWB inequality? The QoLS asks no questions that could address this hypothesis, but the NZGSS has ten variables that may be appropriate. Each variable concerns the respondent's perceptions of the fair treatment of different people by staff from various organisations. Interpreting the analysis for these ten variables individually would be time consuming, and likely not very instructive given that they all theoretically account for the same thing. Therefore, I have created a new binary variable in order to compare those individuals who have a high average perception of fairness in society across these ten variables with those who have a lower average perception of fairness in society.

I discuss the initial variables and the data manipulation process required to create the new variable here. In each year of the NZGSS respondents were asked whether they "think staff at various organisations in New Zealand accept and tolerate different groups" in reference to the following groups: 'staff at your local council', 'the police in your area', 'judges and other staff at law courts', 'staff at government departments', 'your local doctors', 'staff at other health services in your area', 'staff at the schools in your area', 'staff at other education facilities like polytech or university in your area', 'local employers', and 'staff at local shops and other services'. Respondents were given a 5-point scale from 'strongly agree' ('5') to 'strongly disagree' (' 1 ') in response to the statement "(organisation) treat everyone fairly, regardless of what group they are from", where 'organisation' is replaced with one of the above categories. There is a separate variable for each 'organisation'. Unlike the other variables in the NZGSS, a large proportion of each of these questions were coded as 'don't know' ('89').

In order to create a single variable representing an individual's perceptions of fairness in society, the average response of the individual to all ten of the questions was taken. In order to generate this variable, 'don't know' ('89') responses were recoded to be 'neither agree nor disagree' (' 3 ') as this was the response most often statistically not significantly different from 'don't know'. The new 5-point average fairness perception has a mean value of 3.64 in $2008,3.63$ in 2010, and 3.62 in 2012. A binary was created from the average perceptions of fairness variable, with values from ' 1 ' to 
$\mathbf{7 8} \mid \mathrm{P}$ a g e

' 3.49 ' recoded as ' 0 ', and ' 3.5 ' to ' 5 ' recoded as ' 1 '. A total of 5,800 people (67.13\%) perceived organisations to be fair on average in 2008, whilst in 2010 and 2012 these totals were 5,695 (67.13\%) and 5,576 (66.31\%) respectively. As such, a considerable number of people fall into both values of the binary.

Figure 5.4 plots the predicted life satisfaction of those who believe people in society receive fair treatment (top red dashed line) and those who do not (bottom blue solid line). The two separate predictions are generated by interacting the binary 'society is fair' variable with the standard deviation of life satisfaction of the individual's group for each of the three surveys, 2008, 2010, and 2012. The standard errors are adjusted for geographic clustering. The full range of controls from Model 1 to Model 10 have not yet been introduced for this initial picture. 
79 | P a g e

Figure 5.4: Predicted individual life satisfaction by standard deviation life satisfaction by perceptions of fairness, no controls

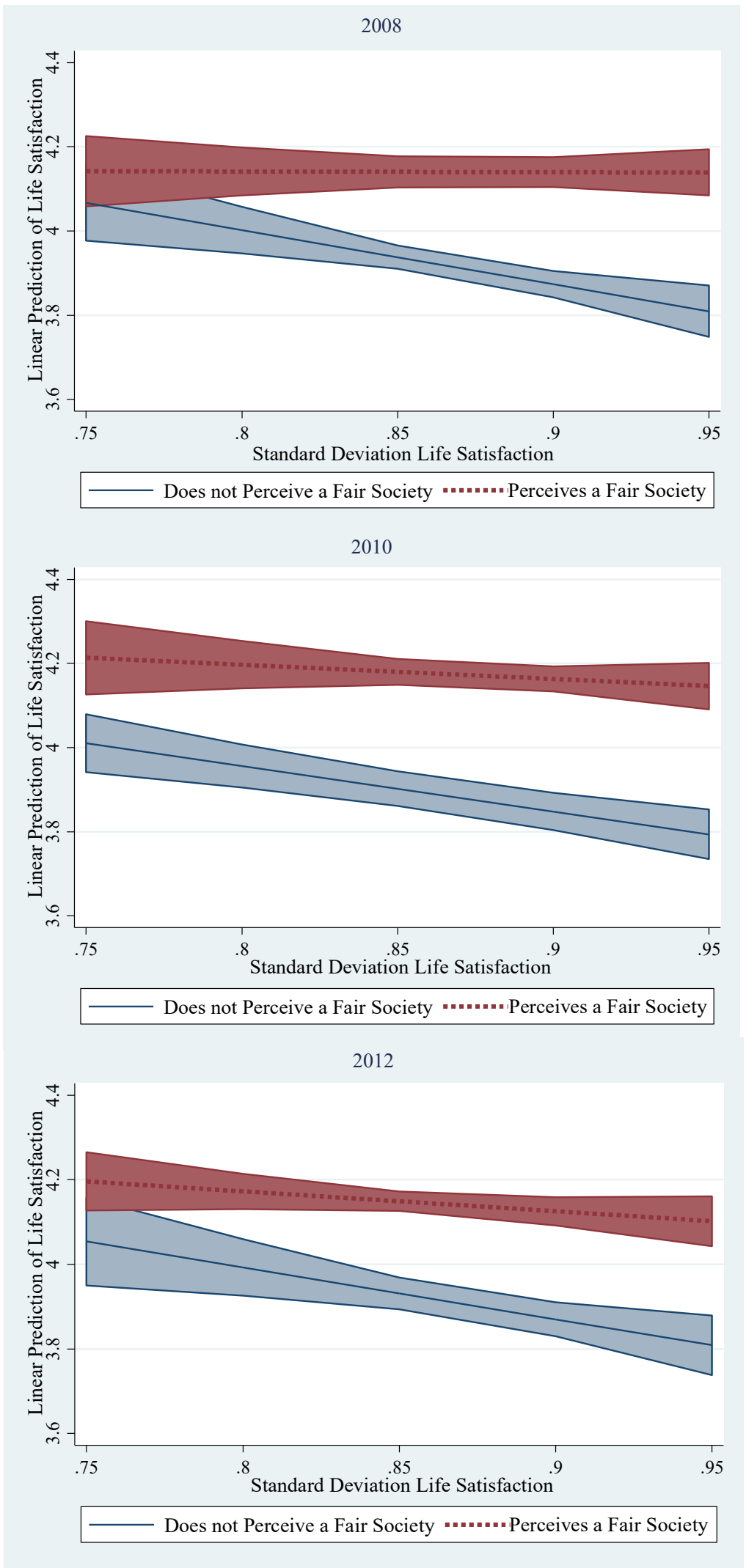

Figure 5.4: NZGSS, 2008, 2010, and 2012, New Zealand 
The underlying regressions for Figure 5.4 reveal more about the differences between those who perceive fairness and those who do not. The results of an interaction in OLS regression can be difficult to understand without an example: as such, Table 5.3 below displays the regression output for the interaction in 2008. The regression indicates that for every hypothetical one unit increase in standard deviation life satisfaction, the life satisfaction of individuals who perceive a fair society is higher by 1.269 on a 5-point scale ( $a=5.030, b=1.269, S E=0.262, p<0.001)$ compared to those who perceive an unfair society. The effect of standard deviation life satisfaction alone is still strong and negative $(a=5.030, b=-1.285, S E=0.341, p<0.01)$.

Looking at the effect of these together, we can tell that the life satisfaction of individuals who perceive an unfair society would fall by -1.285 for every unit increase in standard deviation life satisfaction. In contrast, the life satisfaction of those who perceive a fair society would fall by only -0.016 for every unit increase in standard deviation life satisfaction (as we subtract -1.285 from 1.269). Individuals who perceive society to be fair in 2008 are not only less sensitive to wellbeing inequality, they are almost completely insensitive to wellbeing inequality.

Table 5.3: OLS regression of life satisfaction on the interaction between perceptions of fairness and standard deviation life satisfaction for 2008

\begin{tabular}{|c|c|c|c|c|c|c|}
\hline & Coefficient & SE & $\mathbf{t}$ & $P>t$ & $\begin{array}{r}95 \% \\
\text { In }\end{array}$ & $\begin{array}{l}\text { dence } \\
\text { ls }\end{array}$ \\
\hline SD Life & -1.285 & 0.341 & -3.77 & 0.001 & -1.997 & -0.573 \\
\hline & & & & & & \\
\hline Perceives a Fair & -0.876 & 0.232 & -3.77 & 0.001 & -1.361 & -0.391 \\
\hline & & & & & & \\
\hline $\begin{array}{r}\text { The Interaction } \\
\text { Effect }\end{array}$ & & & & & & \\
\hline Perceives a Fair & 1.269 & 0.262 & 4.84 & 0.000 & 0.723 & 1.815 \\
\hline Society & & & & & & \\
\hline Constant & 5.030 & 0.298 & 16.91 & 0.000 & 4.410 & 5.651 \\
\hline & & 8,576 & & & $\mathrm{R}^{2}=0.0$ & \\
\hline
\end{tabular}

The result for 2008 is evidence my initial predictions may be correct. People who perceive an unfair society experience a greater reduction in their own wellbeing in response to local wellbeing inequality, compared to those who perceive a fair society. 
The following years tell a similar but still different story. In 2010, individuals across New Zealand who perceive society to be fair are still statistically significantly less sensitive to life satisfaction inequality than others $(a=4.824, b=0.748, S E=0.301$, $p<0.05$ ). In 2010 this lower sensitivity does not make them as insensitive to life satisfaction inequality as in 2008 , with the effect of overall standard deviation life satisfaction being greater at $-1.084(a=4.824, b=-1.084, S E=0.236, p<0.001)$. The $\mathrm{R}^{2}$ for 2010 is 0.025 . In 2012, the coefficient of sensitivity to life satisfaction inequality when perceiving a fair society is similar to in 2010 at 0.757 , although this effect is statistically not significant $(p=0.125)$. The coefficient of standard deviation life satisfaction alone in 2012 is $-1.227(p<0.01)$. Such a result suggests that people are sensitive to life satisfaction inequality regardless of their fairness perceptions in 2012.

Although testing The Fairness Hypothesis with the 2012 data gives largely nonsignificant results, it does seem as though there may be some difference in this year. Before exploring these fairness perception results further however, I will consider why we see a strong significant difference according to fairness perceptions in 2008 , and a gradual fall in significance for 2010 and 2012.

In the discussion of The Primary Hypothesis, I touched on the possible impacts of the political climate and macroeconomic conditions on the 2008 data. I believe the difference in sensitivity according to perceptions of fairness is likely due to national politics, and the marked difference in macro conditions over time.

Political debate between the left and right is generally deeply intertwined with inequality. Indeed, Ott (2005) demonstrates that politically left-wing indicators such as expenditure on social security or greater gender empowerment are correlated with lower wellbeing inequality within countries. Those on the political left tend to believe that inequality is present in society, that people are not always treated fairly, and that inequality needs to be addressed. Those on the political right tend to believe that society treats people fairly for the most part and that differences in outcomes are due to merit, luck, or hard work, and thus do not need addressing.

In 2008, New Zealand saw an increase in right wing popularity as the National Party was elected instead of the current Labour Party. Morrison (2015a) cites data that indicates more New Zealanders favour greater income inequality when Labour is in power, and that more favour greater income equality when National is in power. Further, the unemployment rate grew markedly from 2008 to 2010 (Morrison, 2015a), 
$\mathbf{8 2} \mid \mathrm{P}$ a g e

moving from a period where regional variations are typically low to one where they are typically high. This theoretically increased proportion of people seeing society as 'fair enough' at a time of political change could account for the significant difference noted in 2008. In turn, the decreasing significance in difference by perceptions of fairness in 2010 and 2012 could be due to more individuals aligning with a left-wing point of view and becoming more inequality averse due to the increasingly dissatisfactory economic conditions they lived in.

Unfortunately, I can only speculate on the influence of politics on the perceptions of fairness and sensitivity to wellbeing inequality relationship based on literature from the income inequality field. However, determining the influence of political preference and macro conditions on sensitivity to SWB inequality is certainly an area appropriate for future research.

Although I am unable to test for the effects of political orientation, I can test the effects of introducing controls for individual attributes on the relationship between fairness perceptions and sensitivity to wellbeing inequality. Figure 5.5 displays the cluster-controlled interaction between fairness perceptions and sensitivity to life satisfaction in 2008, 2010, and 2012, controlling for all of the variables in Model 10 (i.e. region, sex, ethnicity, age, employment status, higher education, income, ELSI and partnership status).

After introducing controls, the coefficient of the interaction in 2008 is still significant, indicating that those who perceive a fair society are less sensitive to life satisfaction inequality than others $(a=3.474, b=0.773, S E=0.289, p<0.05)$. The effect of standard deviation life satisfaction alone still has a significant negative impact on individual life satisfaction in 2008, even after controls are introduced ( $a=3.474, b=-$ $0.945, S E=0.414, p<0.05)$. The regression therefore predicts that people who perceive society to be fairer (top red line) are barely effected by increases in life satisfaction inequality, holding other things constant. As expected, the $\mathrm{R}^{2}$ is much higher at 0.186 after controls have been introduced. 
Figure 5.5: Predicted individual life satisfaction by standard deviation life satisfaction by perceptions of fairness, with controls
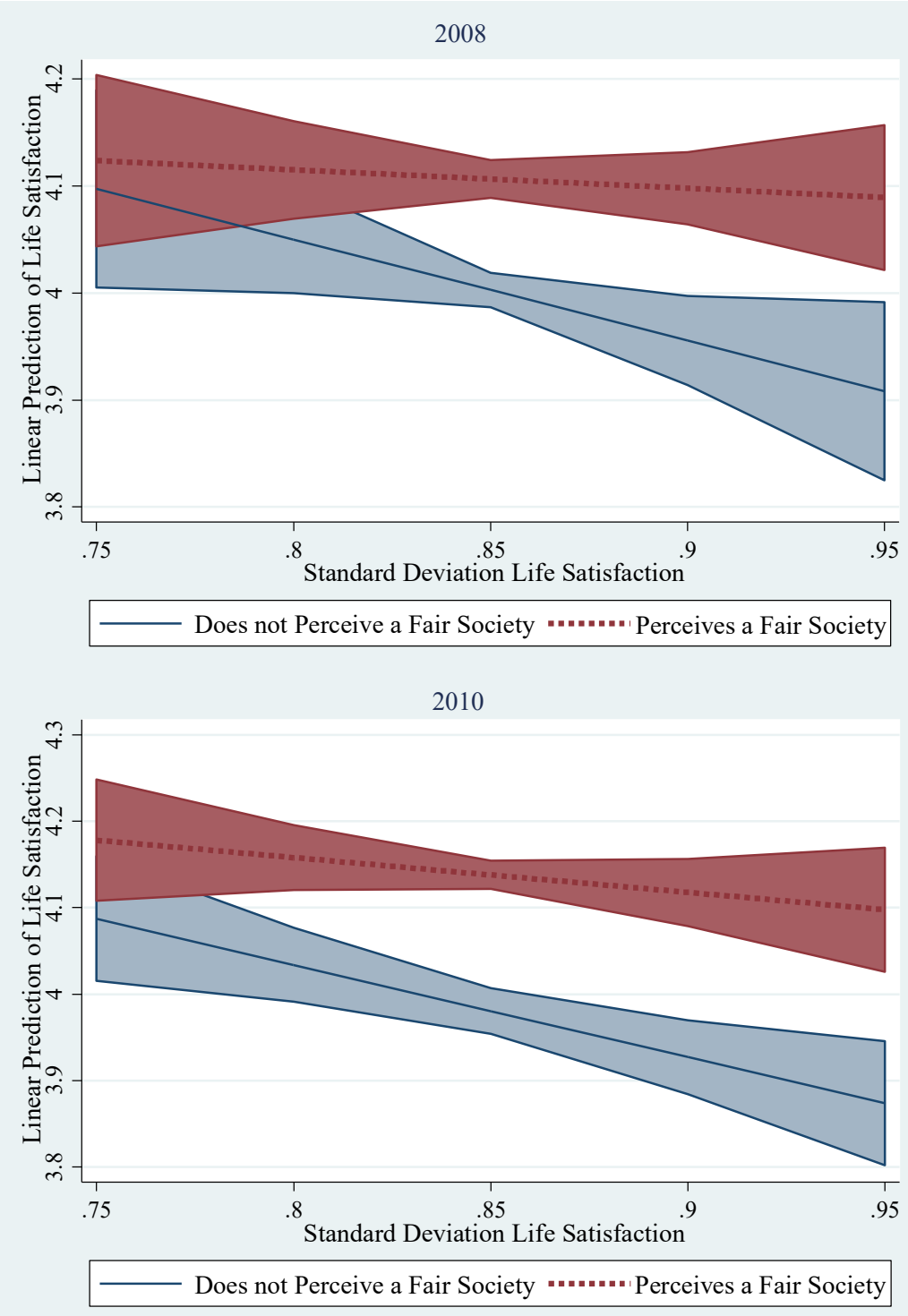

2012

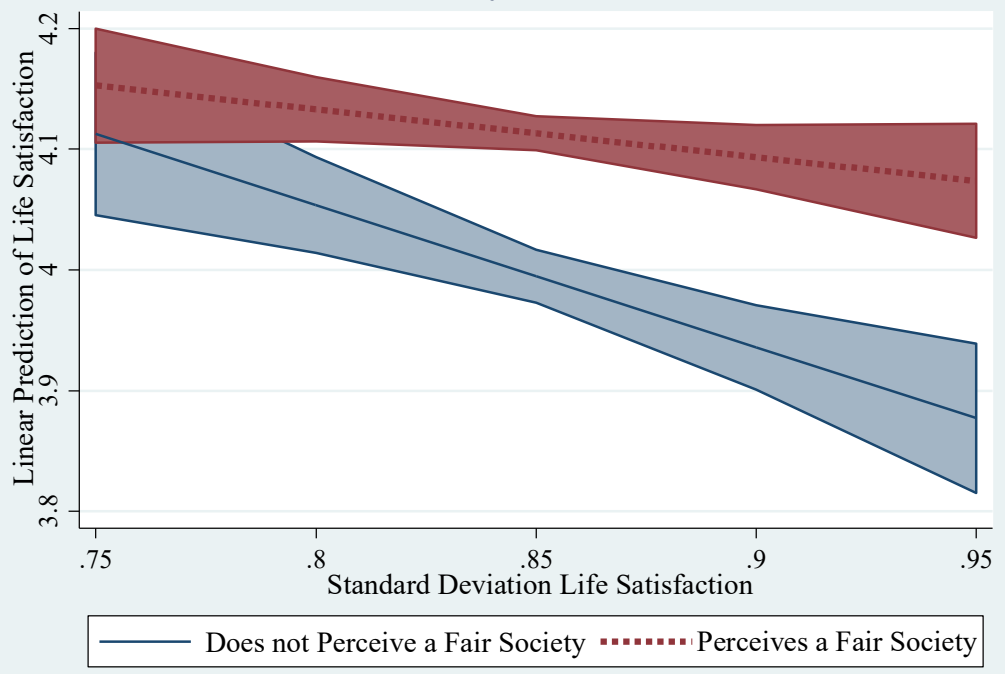

Figure 5.5: NZGSS, 2008, 2010, and 2012, New Zealand 
In 2010, the interaction between life satisfaction inequality and perceiving a fair society is still negative and significant ( $a=3.612, b=0.665, S E=0.286, p<0.05$ ). The effect for 2012 is still not significant ( $p=0.084$ ). A non-significant result for 2012 is in line with the results in section 5.1 that everyone in that year is sensitive to life satisfaction inequality, whereas in 2008 and 2010 sensitivity to life satisfaction inequality is clearest after accounting for fairness perceptions. So far, The Fairness Hypothesis is supported in both 2008 and 2010, but not in 2012 .

When these controls were introduced, the same 13 Models used for the analysis of The Primary Hypothesis were used to generate estimates tables to tell the effect of the interaction between perceptions of fairness and life satisfaction inequality on individual life satisfaction. ${ }^{23}$ Briefly returning to The Primary Hypothesis, I will comment on the effect that controlling for the interaction between standard deviation life satisfaction and perceptions of fairness have upon the relationship between just standard deviation life satisfaction and individual life satisfaction, rather than repeating the results for all of the controls.

With controls in place in 2008, the relationship between life satisfaction and life satisfaction inequality becomes statistically significant in all models up to Model 10. In Model 13 after subjective controls are introduced the effect is still negative at -0.746 , but it misses out on statistical significance $(p=0.086)$.

In 2010, the effect of life satisfaction inequality on individual life satisfaction becomes significant in all models with the introduction of controls. The slope coefficient falls from $-1.084(a=4.824, b=-1.084, S E=0.236, p<0.001)$ in Model 1 to a surprisingly similar value of $-0.947(a=3.309, b=-0.946, S E=0.299, p<0.01)$ in Model 13 after subjective controls have been introduced.

In 2012, the relationship is significant in all models except for Model 13 when subjective social isolation is introduced (one of the strongest correlates of SWB). The coefficient of standard deviation life satisfaction's effect on individual life satisfaction falls sharply from Model $10(a=3.619, b=-1.177, S E=0.292, p<0.01)$ to Model 12 $(a=3.147, b=-0.664, S E=0.283, p<0.05)$. In Model 13 the effect is negative, but misses out on significance by a small margin $(p=0.061)$.

\footnotetext{
${ }^{23}$ Due to space constraints the estimates tables are not presented here.
} 
As such, the negative effect of local life satisfaction inequality is significant in 2008, 2010, and 2012 when accounting for perceptions of fairness and a variety of nonendogenous control variables.

Returning to The Fairness Hypothesis, the final factor worth exploring is the impact that objective disadvantage might have on the relationship between an individual's perceptions of fairness and their sensitivity to SWB inequality. The NZGSS codes each individual with a New Zealand Deprivation Index ranking from 0 (least deprived) to 10 (most deprived) ${ }^{24}$ for the area they live in, allowing the analysis of The Fairness Hypothesis according to groups from areas of different deprivation levels. It is reasonable to assume that individuals living in more deprived areas who also perceive society to be unfair may be more sensitive to SWB inequality in their area, as they would likely be more aware of disadvantage in society. As such, I reran the cluster-controlled interaction between the standard deviation of life satisfaction and fairness perceptions for each year, splitting the data into those 'most deprived' (9 or 10 on the New Zealand Deprivation Index) and everyone else (8 or lower). All regressions include the non-endogenous controls as specified in Model 10.

The regression results here are inconsistent in each year. In 2008, controlling for other factors, those most deprived $(\mathrm{N}=1,405)$ are actually not statistically significantly sensitive to life satisfaction inequality in general $(p=0.838)$ or as a result of their perceptions of fairness $(p=0.570)$. It is everyone else $(\mathrm{N}=7,630)$ who are significantly negatively impacted by life satisfaction inequality around them $(a=3.481$, $b=-0.977, S E=0.363, p<0.05)$. It is for this latter group that perceptions of fairness play a part: individuals who perceive a fair society are less sensitive to life satisfaction inequality $(a=3.481, b=-0.906, S E=0.264, p<0.01)$. The $\mathrm{R}^{2}$ value is 0.180 . It appears as though individuals living in high deprivation locations in 2008 are not sensitive to SWB inequality in their area.

In 2010, neither high deprivation areas $(\mathrm{N}=1,378)$ nor lower deprivation areas $(\mathrm{N}=7,439)$ demonstrate a significant relationship between perceptions of fairness and standard deviation life satisfaction. Those in high deprivation areas again appear not to be statistically significantly sensitive to SWB inequality $(p=0.917)$. Changing the cut

\footnotetext{
${ }^{24}$ For more information on the New Zealand Deprivation Index, see https://www.otago.ac.nz/wellington/departments/publichealth/research/hirp/otago020194.html
} 
off points of the New Zealand Deprivation groups does not alter the lack of statistical significance in 2010 .

In 2012, I find a contradiction of the findings in 2008 and 2010. It appears that deprivation in 2012 may have masked some of the influence of perceptions of fairness on sensitivity to life satisfaction inequality. Those living in high deprivation areas $(\mathrm{N}=1,847)$ who perceive society to be fair when interacted with life satisfaction inequality demonstrate a substantively large coefficient of $2.748(a=4.371, b=2.748$, $S E=0.510, p<0.001)$. The coefficient for the interaction dwarfs that for standard deviation life satisfaction alone at $-2.041(a=4.371, b=-2.041, S E=0.663, p<0.01)$, indicating that those who perceive a fair society and live in a high deprivation area actually get more satisfied as local life satisfaction inequality increases. The $\mathrm{R}^{2}$ for this regression is 0.223 . The interaction for the less deprived portion of the population $(\mathrm{N}=7,162)$ is statistically not significant $(p=0.353)$, although the effect of life satisfaction inequality alone remains strong $(a=3.475, b=-1.051, S E=0.374, p<0.05)$.

The results of the regression for the high deprivation locations (9 or 10 on the index) in 2008, 2010, and 2012 are plotted in Figure 5.6. The trends in Figure 5.6 may seem odd at face value: in 2012 people living in deprived areas who also perceive a fair society are more satisfied when there is greater inequality in life satisfaction. In contrast, people living in high deprivation areas in 2008 and 2010 seem to largely not be sensitive to life satisfaction inequality in their locality.

However, the 2012 results do still support The Fairness Hypothesis, as those who perceive society to be fair are being less sensitive to SWB inequality. The lack of sensitivity in deprived areas in 2008 and 2010 again could be due to changes in macroeconomic conditions (which would affect those most deprived the worst). However, it could be that the unexpected results are due to the smaller $\mathrm{N}$ in high deprivation areas. Regardless, the fact that some individuals actually seem to benefit from SWB inequality when they perceive a fair society is one that warrants investigation in future research. 
87 | P a g e

Figure 5.6: Predicted individual life satisfaction by standard deviation life satisfaction by perceptions of fairness, high deprivation areas
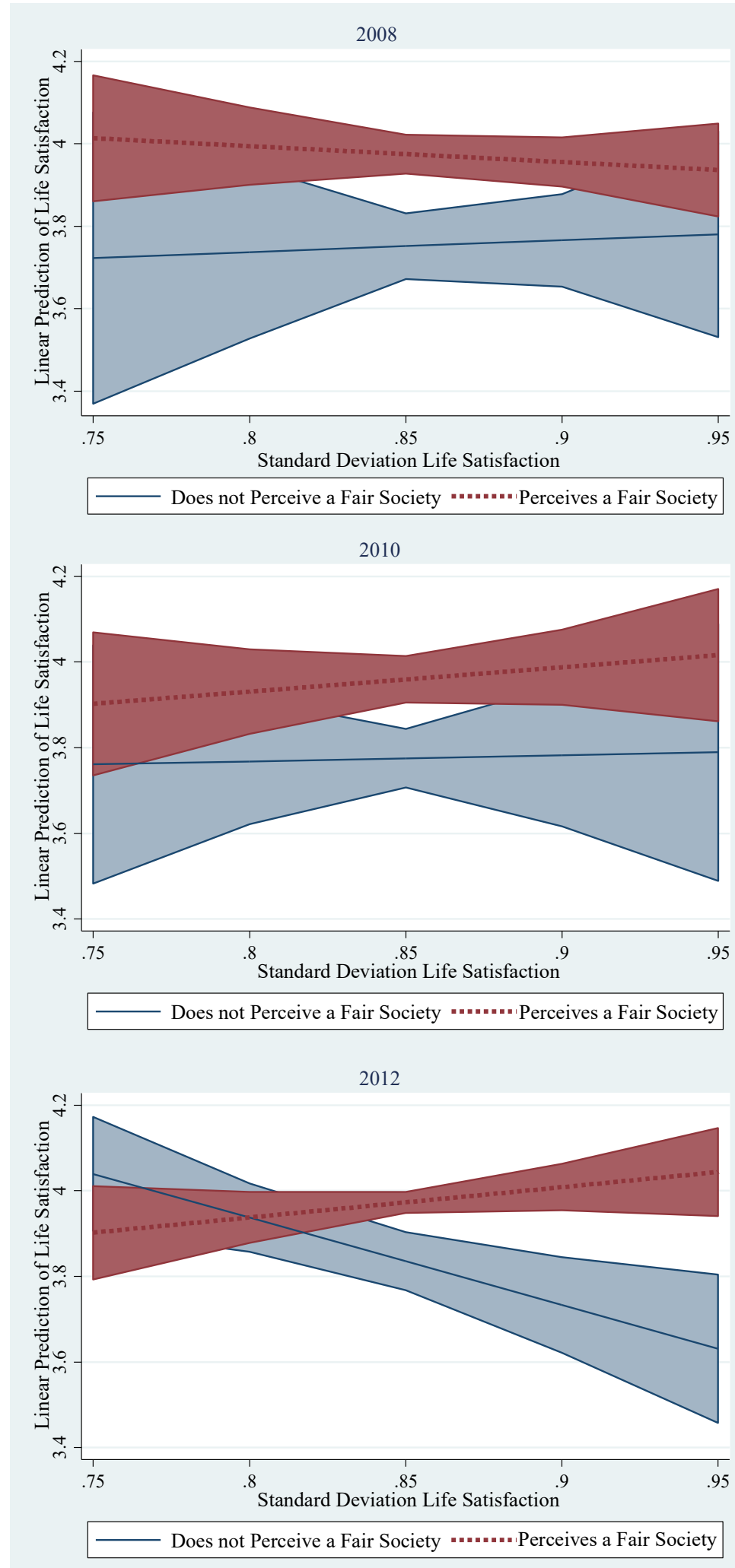

Figure 5.6: NZGSS, 2008, 2010, and 2012, New Zealand. High deprivation areas encompass those scoring a 9 or a 10 on the New Zealand Deprivation Index. 
Overall, The Fairness Hypothesis is largely supported by my results. In 2008, I find consistent support for The Fairness Hypothesis, with those who perceive society to be fair being less sensitive to wellbeing inequality. The exception to this is for those most deprived in New Zealand when controlling for other factors, when there is no significant difference according to fairness perceptions.

In 2010, the results support the fairness hypothesis both with and without controls. The exception to this is when the population is split according to their score on the New Zealand Deprivation Index, where perceptions of fairness no longer appear to impact sensitivity to wellbeing inequality.

In 2012, the results largely do not support The Fairness Hypothesis, as people seemed sensitive to wellbeing inequality regardless of their perceptions. The interactions between perceptions of fairness and wellbeing inequality are only statistically significant when running the analysis for those living in the highest deprivation areas of New Zealand.

While the results for The Fairness Hypothesis are far from conclusive, they certainly demonstrate that perceptions of fairness play a role in affecting some individuals' sensitivity to SWB inequality. Controlling for the interaction of fairness perceptions and wellbeing inequality also seems to increase the effect of just wellbeing inequality on individual wellbeing.

In the following chapter I analyse The Fairness Hypothesis further using a different dataset, providing greater clarity regarding the impact of individual fairness perceptions. Regardless, future research on the relationship between SWB and SWB inequality would do well to explore the impact of perceptions of fairness.

An individual's perceptions of fairness may not be the only thing effecting their sensitivity to life satisfaction inequality. The following section addresses whether altruistic virtues may have an impact on the relationship between wellbeing and wellbeing inequality.

\section{3: The Altruism Hypothesis}

Altruism involves an individual going out of their way to do something that benefits others, particularly if there is no direct benefit for themselves. Other research has found that participating in altruistic activities is beneficial for the wellbeing of 
individuals (Becchetti et al., 2017; Binder, 2015). However, the effect of altruism on SWB inequality or sensitivity to it has not yet been explored.

My altruism hypothesis predicts that more altruistic individuals will be more sensitive to inequality in SWB than less altruistic individuals. While none of the surveys used in this thesis contain specific measures of altruistic values, the NZGSS has variables which may be used as proxies.

The NZGSS contains variables on helping others and volunteering, and I take these altruistic actions as likely representative of altruistic values, as others have done before me (Becchetti et al., 2017). Furthermore, there is some evidence that females are more altruistic than males and that the elderly are more altruistic in their preferences for equality than younger individuals (Morrison, 2015b; Rand et al., 2016; Simmons \& Emanuele, 2007). As such, sex and age are also used as a test of the altruism hypothesis.

I begin with sex, testing the difference between male and female sensitivity to SWB inequality. As females are theoretically more altruistic, I expect them to be more sensitive to SWB inequality. Running interactions of sex and standard deviation life satisfaction revealed no significant difference at the individual level for any of the years. In all cases, the sensitivity of males and females were statistically indistinguishable, both with and without controls.

Testing age as a proxy for altruism tells a similar story. For the purposes of this hypothesis, I created a new age variable to test the difference between younger, middleaged, and older age groups. Those who are in the age groups covering 15 years to 29 years were grouped into the younger age category, those in the 30 to 59 years age groups were grouped into the middle-aged category, and those 60 years and older were placed in the older age category. Rather than creating a simple binary variable, a 3-point variable was created to account for the established U-shaped relationship between age and wellbeing (Dolan et al., 2008). Based on evidence that older individuals prefer greater income equality (Morrison, 2015a), I expect them to be more sensitive to inequalities in wellbeing around them. Interacting standard deviation life satisfaction in an individual's area with this new 3-point age group categorical variable reveals no difference between age groups in 2008, 2010, and 2012, either with or without controls.

Luckily, the NZGSS contains some more common proxies for altruism: one variable representing volunteering, and another representing providing unpaid help to 
$\mathbf{9 0} \mid \mathrm{P}$ a g e

someone outside your household. Interactions between each of these altruism variables and standard deviation life satisfaction were run for each year. The same 13 Models used to analyse The Primary Hypothesis were used again here to determine the effect of controls on how the interaction between SWB inequality and altruism effects individual wellbeing.

The interaction of volunteering and life satisfaction inequality does not have a significant effect on individual life satisfaction in any of the 13 Models run in 2008, 2010, or 2012. Likewise, the interaction of providing unpaid help and life satisfaction inequality has no significant effect in any year for any Model.

Why do these theoretically closer proxies of altruism also exhibit no effect on individual sensitivity to wellbeing inequality? There is evidence that volunteering protects individual SWB from adverse circumstances (Binder, 2015). It is therefore possible that performing altruistic actions such as volunteering actually protects individuals from the negative effects of inequality around them, cancelling out the greater sensitivity I expected. Further, Becchetti and colleagues (2017) demonstrate that altruistic values do not always underlie theoretically altruistic actions like volunteering: this phenomenon could also explain the lack of effect altruism has on sensitivity to SWB inequality in the NZGSS.

Regardless, The Altruism Hypothesis is not supported using data from the NZGSS. It may be that the lack of results gained using this dataset are a result of the indicators used to represent altruism. Chapter 6 will re-test The Altruism Hypothesis using measures from a different survey. Chapter 7 will suggest avenues for future research that could address The Altruism Hypothesis.

\section{4: Summary}

I set out this chapter with the goal of explaining why a negative relationship between wellbeing and local inequalities in wellbeing exists, drawing on the NZGSS. I offered forth two possible explanations for this sensitivity - perceptions of fairness and altruism - which I have since tested.

I began with by using the NZGSS to retest the hypothesis that wellbeing is sensitive to local wellbeing inequality. I find a consistent negative relationship between life satisfaction and life satisfaction inequality at the ecological level. The negative relationship is consistent at the individual level as well, although there is evidence that 
91 | P a g e

sensitivity to wellbeing inequality may differ between years (according to macroeconomic conditions) and according to personal characteristics or values. While the NZGSS is not an ideal dataset for testing The Parochialism Hypothesis owing to the geographic indicators it contains, the evidence from section 5.1 supports the hypothesis by indicating that sensitivity to wellbeing inequality is greater at smaller scales.

In testing The Fairness Hypothesis, I assessed how perceptions of fair treatment in society might change an individual's sensitivity to the inequality in wellbeing around them. Support for this hypothesis is mixed. In 2008, a time of political change for New Zealand, those who perceive society to be fair are less sensitive to wellbeing inequality, supporting The Fairness Hypothesis. In 2010, after a two year period of economic turmoil, those who perceive society to be fair are still less sensitive to inequality than those who perceive an unfair society, but this difference is smaller in magnitude. In 2012, The Fairness Hypothesis is supported only among those living in the highest deprivation areas of New Zealand, where those who perceive fairness got more satisfied as inequality rises.

There is evidence that controlling for the interaction between wellbeing inequality and perceptions of fairness makes the effect of solely wellbeing inequality on individual wellbeing clearer. Taken together, these results indicate that perceptions of fairness almost certainly have some influence on an individual's sensitivity to wellbeing inequality.

The Altruism Hypothesis involved testing whether typically altruistic groups are more sensitive to life satisfaction inequality than others. The altruistic groups tested were females, older age groups, volunteers, and those who provided unpaid help to others. No significant difference was found in sensitivity to wellbeing inequality between any of the more altruistic and less altruistic groups. The issue may lie in the variables used to represent altruism, or it could be the case that altruism has no effect.

The following chapter will draw on Te Kupenga to explore the sensitivity of Māori to local wellbeing inequality. Chapter 6 will also retest both The Fairness Hypothesis and The Altruism Hypothesis. 


\section{Chapter 6: Do These Mechanisms Apply for Māori?}

I have already analysed two large New Zealand datasets, so why analyse Te Kupenga? First and foremost, the survey represents a unique opportunity to determine whether the negative relationship between wellbeing and wellbeing inequality exists for the Māori subset of the population which is generally a minority in other surveys. Furthermore, drawing on Te Kupenga allows me to test whether the same mechanisms for non-Māori (fairness perceptions and altruism) might underlie sensitivity to wellbeing inequality for Māori. Given that Māori generally have lower life satisfaction than non-Māori (Sibley et al., 2011), and that Māori wellbeing is different conceptually from non-Māori wellbeing (Cram, 2014), specifically exploring the way that Māori are impacted by local inequality in the wellbeing of other Māori is worthwhile.

Te Kupenga is New Zealand's first nationally representative survey of Māori wellbeing, and was conducted by Statistics New Zealand following the 2013 census. The survey collects information on the cultural, social and economic wellbeing of Māori in New Zealand. Te Kupenga draws on a range of measures used in other surveys (such as the NZGSS and the World Values Survey), but also uses a number of new questions specifically relating to key aspects of Te Ao Māori, such as wairuatanga (spirituality), tikanga (Māori customs and practices), whanaungatanga (social connectedness) and te reo Māori. By focusing solely on Māori, Te Kupenga holds some cultural and personal characteristics relatively constant, allowing for the analysis of the sensitivity of Māori to the wellbeing of other Māori in their area ${ }^{25}$.

I begin by asking whether the relationship between SWB and SWB inequality is as strong or stronger when drawing from a Māori sample. I will then analyse both The Fairness Hypothesis and The Altruism Hypothesis, expecting both to play a more prominent role for Māori specifically than for the general population in Chapter 5.

\footnotetext{
${ }^{25}$ Before I proceed, it is important I recognise my positionality in regards to the research I am conducting. As a New Zealand European with no Māori upbringing and only an outsider's familiarity with Māori culture, I must recognise that I approach this research from a distinctly non-indigenous viewpoint. I do not pretend to be an expert on Te Ao Māori, nor do I claim to be speaking for Māori in my interpretation. While emphasising researcher positionality is an unconventional practice in quantitative research, I feel it is important to acknowledge that I can only present the following results from my perspective as a New Zealand European, whereas a Māori researcher would likely be able to do the data greater justice in their interpretation.
} 


\section{1: The Primary Hypothesis - Does the Wellbeing of Māori Decline with the Inequality of Wellbeing Among Māori?}

Is the wellbeing of Māori related to sub-national wellbeing inequality among Māori in New Zealand? One benefit of using Te Kupenga to test my hypotheses is that the life satisfaction measure is on an 11-point scale ranging from 0-10, allowing for more spread in wellbeing than in the previous two surveys with only a 5-point scale ${ }^{26}$. The specific life satisfaction question asked in Te Kupenga was "Where zero is completely dissatisfied, and ten is completely satisfied, how do you feel about your life as a whole?".

The unusually high level of mean life satisfaction in Te Kupenga is worth discussing. Summary statistics reveal a mean life satisfaction of 7.744 across the entirety of the dataset. This value places Māori sampled in Te Kupenga above the New Zealand population mean of 7.334 as reported by Helliwell and colleagues (2016) on a similar 0-10 scale of life satisfaction ${ }^{27}$. At first this seems to contradict Sibley and colleagues' (2011) finding that the SWB gap between Māori and New Zealand Europeans has widened over time. However, within the Te Kupenga sample, Māori who also identity as New Zealand European report higher mean life satisfaction (7.803) than Māori who do not identify as New Zealand European (7.673). A t-test indicates the means are statistically significantly different $(p<0.01)$, highlighting the need for a better understanding of the cultural factors underlying self-representation in surveys, especially for Māori.

Like the NZGSS, Te Kupenga is used in its confidentialised unit record files (CURFs) format. Many of the variables in the CURFs are collapsed into a smaller number of categories in order to protect the confidentiality of the respondent. The urban variable and the region variables are two which were collapsed, representing a barrier for testing the relationship between SWB and SWB inequality across these places.

In order to generate a more nuanced geographic variable, I have followed the same process as in the NZGSS and have combined the region variable with the urban

\footnotetext{
${ }^{26}$ A convincing argument has been made that whānau wellbeing is as good (if not better) as a measure of SWB for Māori (for a review of whānau wellbeing as it appears in Te Kupenga, see Kukutai, Sporle \& Roskruge, 2017). However, in order to maintain consistency with the previous analyses in an explicit focus on individual wellbeing, analysis is presented here only for the life satisfaction variable.

${ }^{27}$ It is worth noting here that the discrepancy between these values may be due to the conceptual differences between the Cantril Ladder measure used by Helliwell and colleagues and the life satisfaction question used in Te Kupenga.
} 
$94 \mid \mathrm{P}$ a g e

indicator to make an urban-region variable. Unfortunately, there are only three urban indicators rather than four like in the NZGSS. As such, a total of only 18 urban-region geographic categories were able to be created.

Table 6.1 displays labels (top row) for these new categories as they will appear on future graphs. The mean life satisfaction on the $0-10$ scale is also presented for each group in Table 6.1, as are the standard deviation life satisfaction values (in brackets). The size of each group $(\mathrm{N})$ is provided in each cell as well.

Table 6.1: Details for the Te Kupenga urban-region variable

\begin{tabular}{|c|c|c|c|}
\hline & Main Urban & Other Urban & Rural \\
\hline $\begin{array}{r}\text { North of North } \\
\text { Island }\end{array}$ & $\begin{array}{c}\text { N1 } \\
7.715 \\
(1.815) \\
\mathrm{N}=850\end{array}$ & $\begin{array}{c}\mathbf{N} 2 \\
7.939 \\
(1.740) \\
\mathrm{N}=573\end{array}$ & $\begin{array}{c}\mathbf{N 3} \\
7.986 \\
(1.628) \\
\mathrm{N}=420\end{array}$ \\
\hline Auckland Region & $\begin{array}{c}\text { A1 } \\
7.592 \\
(1.749) \\
\mathrm{N}=910\end{array}$ & $\begin{array}{c}\mathbf{A 2} \\
7.732 \\
(1.633) \\
\mathrm{N}=82\end{array}$ & $\begin{array}{c}\mathbf{A 3} \\
7.750 \\
(1.041) \\
\mathrm{N}=28\end{array}$ \\
\hline $\begin{array}{r}\text { Middle North } \\
\text { Island }\end{array}$ & $\begin{array}{c}\text { M1 } \\
7.594 \\
(1.929) \\
\mathrm{N}=807\end{array}$ & $\begin{array}{c}\text { M2 } \\
7.674 \\
(2.041) \\
\mathrm{N}=291\end{array}$ & $\begin{array}{c}\text { M3 } \\
7.940 \\
(1.667) \\
\mathrm{N}=184\end{array}$ \\
\hline Wellington Region & $\begin{array}{c}\text { W1 } \\
7.937 \\
(1.737) \\
\mathrm{N}=494\end{array}$ & $\begin{array}{c}\text { W2 } \\
7.849 \\
(2.137) \\
\mathrm{N}=106\end{array}$ & $\begin{array}{c}\text { W3 } \\
8.733 \\
(1.335) \\
N=15\end{array}$ \\
\hline Canterbury Region & $\begin{array}{c}\text { C1 } \\
7.697 \\
(1.827) \\
\mathrm{N}=244\end{array}$ & $\begin{array}{c}\text { C2 } \\
7.738 \\
(1.836) \\
\mathrm{N}=103\end{array}$ & $\begin{array}{c}\text { C3 } \\
7.815 \\
(1.570) \\
\mathrm{N}=27\end{array}$ \\
\hline Rest of South Island & $\begin{array}{c}\text { S1 } \\
7.449 \\
(1.847) \\
\mathrm{N}=247\end{array}$ & $\begin{array}{c}\text { S2 } \\
7.852 \\
(1.490) \\
\mathrm{N}=88\end{array}$ & $\begin{array}{c}\text { S3 } \\
7.875 \\
(1.956) \\
N=64\end{array}$ \\
\hline
\end{tabular}

Of the above categories, the rural Auckland region (A3), the rural Wellington region (W3), and the rural Canterbury region (C3) have sample sizes below 30. As 
95|P a g e

such, they do not allow enough variation to get an effective measure of life satisfaction inequality, and will be excluded from the analysis.

Although I have created this urban-region variable, I will begin with analysis of the relationship between mean and standard deviation life satisfaction in the six regions of New Zealand included in the survey. Figure 6.1 displays a scatter of this relationship.

Figure 6.1: The relationship between mean and standard deviation life satisfaction by New Zealand regions

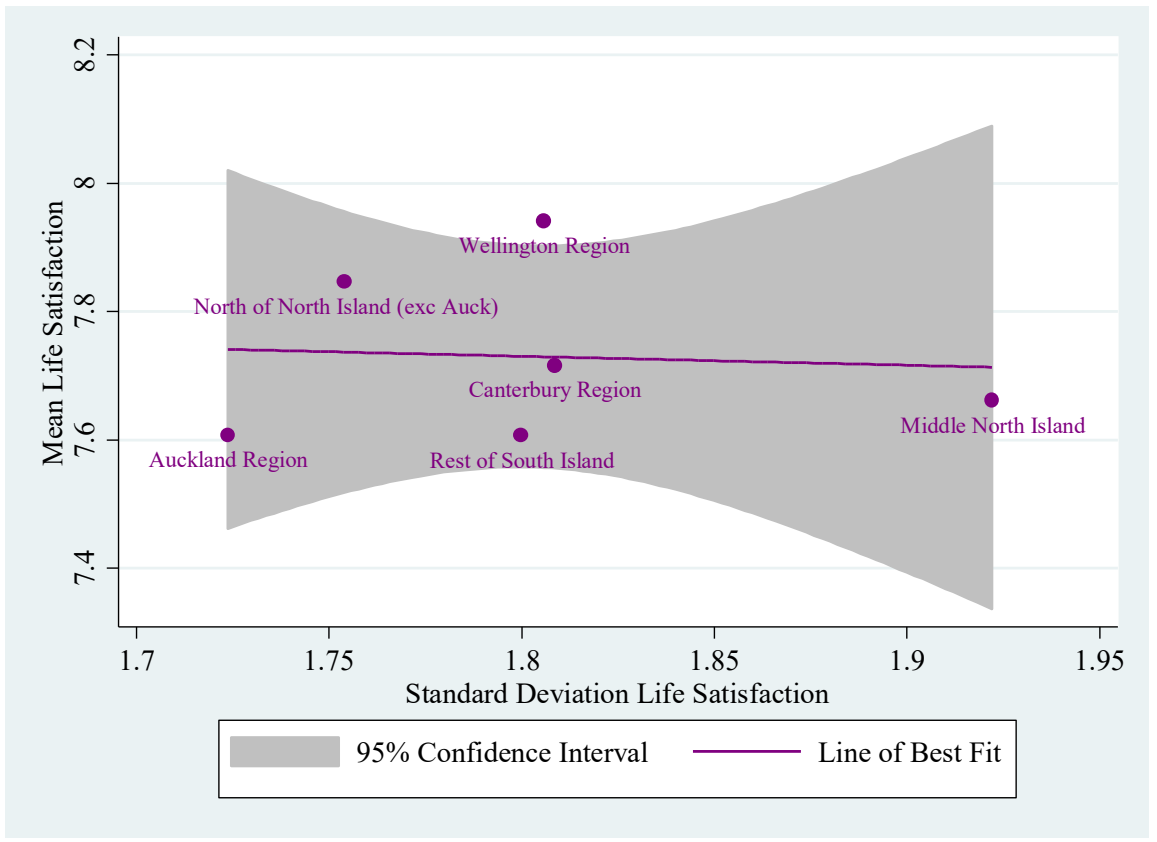

Figure 6.1: Te Kupenga, 2013, New Zealand

There is no evidence of a relationship between mean and standard deviation life satisfaction among Māori at the level of the region in New Zealand ( $p=0.896)$. Notably, with an $\mathrm{R}^{2}$ of only 0.005 , this model explains very little of the variation in life satisfaction compared to the majority of ecological regressions presented in earlier chapters. This suggests that average Māori life satisfaction in regions is not sensitive to regional Māori life satisfaction inequality.

This relative lack of sensitivity may be due to the large size of the regions. Like in the NZGSS, I may find that sensitivity to SWB inequality is uncovered only when conducting analysis at a smaller sub-national scale. Before running the ecological regressions using the urban-region indicator, I briefly check their standard deviation values for outliers. A box plot (not presented here) indicates that three of the remaining categories are outliers (other urban middle North Island: M2, other urban Wellington: W2, and other urban rest of South Island: S2). The analysis in Chapter 4 indicated that 
$\mathbf{9 6} \mid \mathrm{P}$ a g e

outliers are likely to skew the reality of the relationship between life satisfaction and life satisfaction inequality, and so the outliers are excluded prior to the analysis.

Figure 6.2 plots the relationship between mean and standard deviation life satisfaction using the 12 urban-region geographic categories. Once again it seems as though the life satisfaction of Māori are not sensitive to the life satisfaction inequality among Māori within New Zealand. While the coefficient for this relationship is negative at -0.724 , it is not significant, with a $p$-value of 0.137 . The Primary Hypothesis is not supported on the basis of the ecological relationships presented thus far.

Figure 6.2: The relationship between mean and standard deviation life satisfaction by urban-region categories

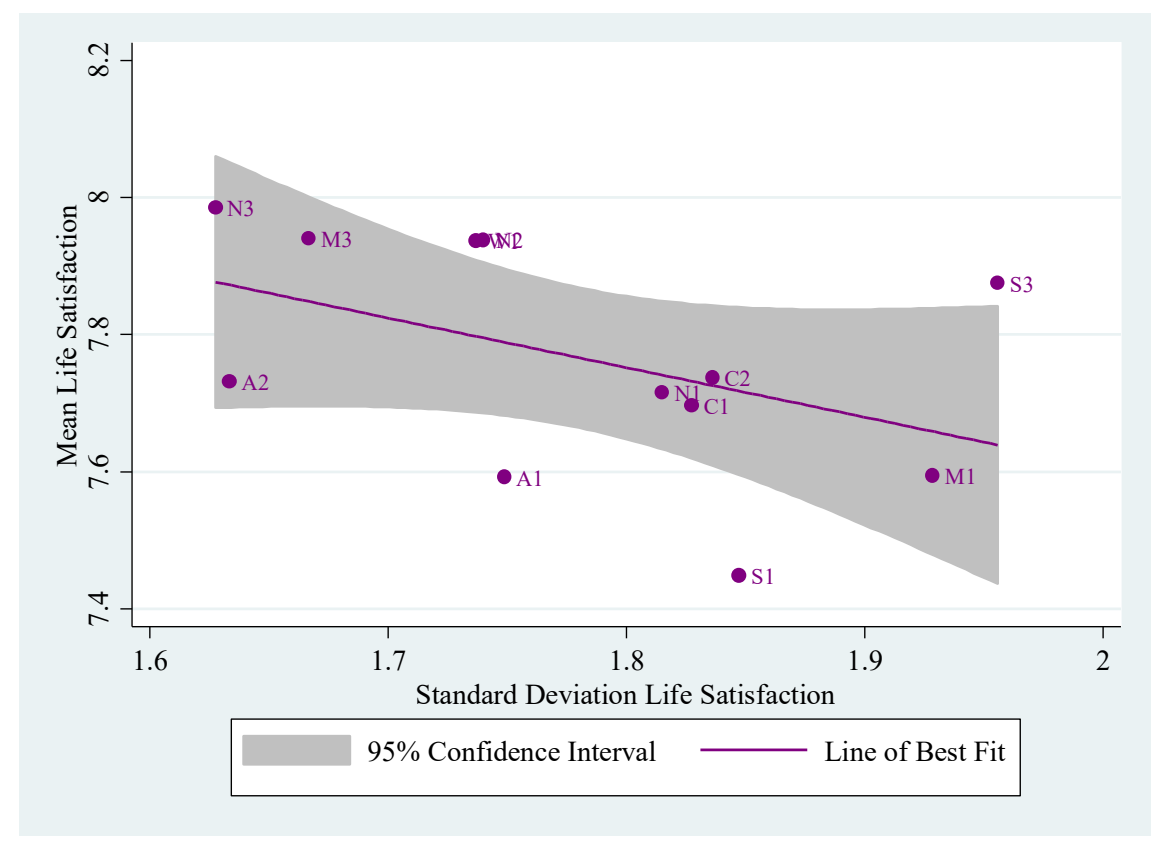

Figure 6.2: Te Kupenga, 2013, New Zealand

The urban-region categories in Table 6.1 are of widely varying size, meaning that some smaller geographic areas may be skewing the results. Therefore, I re-present Figure 6.2 using frequency weights representing the population of respondents within groups. The markers in the resulting Figure 6.3 have been replaced with hollow circles corresponding to the relative sample size of the geographic group. A larger circle is proportional to the square root of the population size.

The complementary weighted regression indicates a strong statistically significant negative relationship between life satisfaction and life satisfaction inequality by group $(a=9.489, b=-1.181, S E=0.020, p<0.001)$. The $\mathrm{R}^{2}$ value of 0.407 indicates that 
97| P a g e

the inequality in life satisfaction accounts for just under half of the variance in mean life satisfaction across the 12 areas. $^{28}$

\section{Figure 6.3: The frequency weighted relationship between mean and standard} deviation life satisfaction by urban-region categories

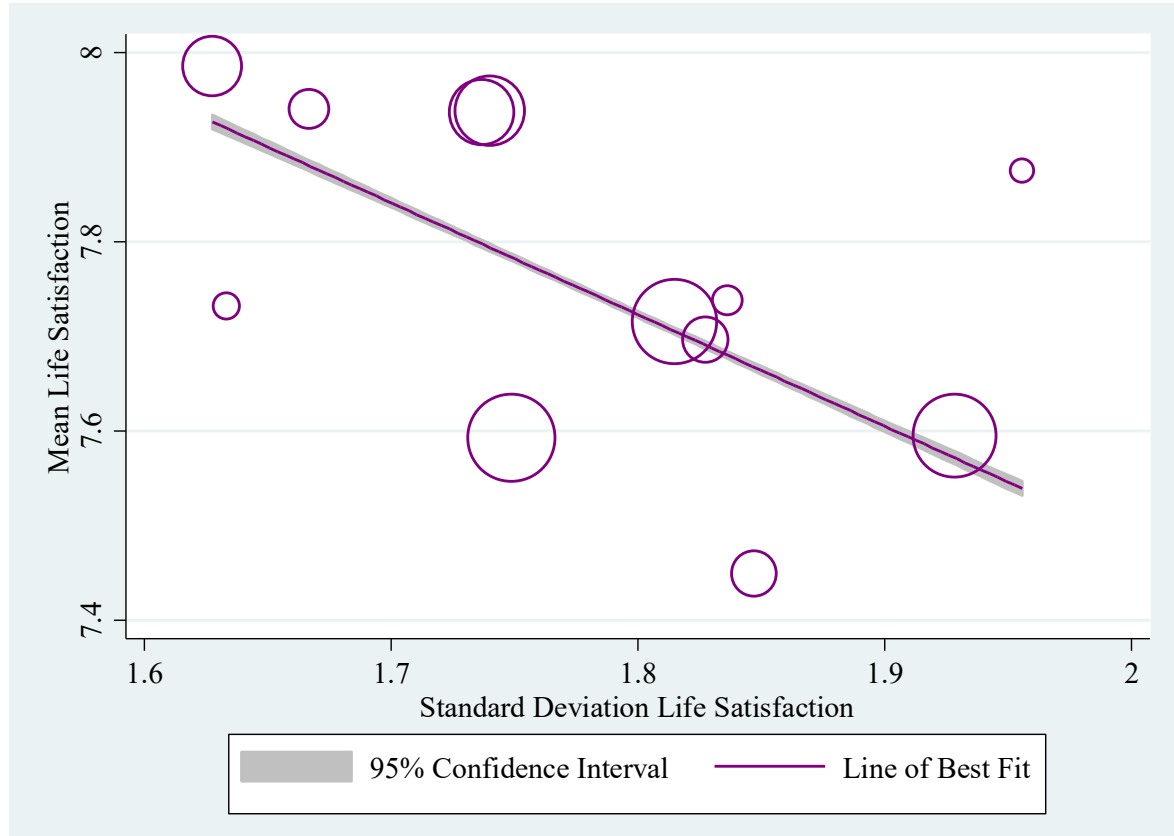

Figure 6.3: Te Kupenga, 2013, New Zealand

The coefficient of the slope in the weighted regression ought to correspond exactly to the coefficient of the un-controlled regression in the individual level model. Table 6.2 contains ten cluster-controlled models of the relationship between standard deviation life satisfaction and individual life satisfaction. With each model, a new control is added to the relationship. Model 1 examines the impact of standard deviation life satisfaction in an individual's area on their own life satisfaction. Model 2 introduces a control for the respondent's region. Models 3 through 5 control for demographic characteristics (sex, age group, and a binary variable indicating if the respondent identifies as New Zealand European in addition to being Māori). Models 6 through 10 control for achieved or chosen characteristics (partnership status, employment status, income, the New Zealand Deprivation Index, and smoking status). Models 11 and 12 introduce subjective health and a measure of social isolation respectively. As in Chapter 4 and Chapter 5, these subjective controls will be considered separately owing to their known high correlation with wellbeing.

\footnotetext{
${ }^{28}$ To compare the impact that the potential outliers would have upon the relationship, I reanalysed the weighted ecological regression with the outlier groups included. The coefficient is far weaker at -0.587 $(p<0.001)$, and the $\mathrm{R}^{2}$ falls by almost half to 0.221 . Such a result indicates that their exclusion from the analysis is sensible.
} 
Table 6.2: Models of individual life satisfaction against standard deviation life satisfaction of urban-region group, 2013 (cluster-controlled)

\begin{tabular}{|c|c|c|c|c|c|c|c|c|c|c|}
\hline & Model 1 & Model 2 & Model 3 & Model 4 & Model 5 & Model 6 & Model 7 & Model 8 & Model 9 & Model 10 \\
\hline SD Life Satisfaction & $-1.181 * *$ & $-1.253 * * *$ & $-1.253 * * *$ & $-1.240 * * *$ & $-1.282 * * *$ & $-1.038 * * *$ & $-1.012 * * *$ & $-1.030 * * *$ & $-.999 * *$ & $-1.028 * *$ \\
\hline & & & & & & & & & & \\
\hline \multicolumn{11}{|l|}{$\begin{array}{r}\text { Region (Base North of } \\
\text { North Island) }\end{array}$} \\
\hline Auckland & & $-.255 * * *$ & $-.255 * * *$ & $-.252 * * *$ & $-.259 * * *$ & $-.249 * * *$ & $-.244 * * *$ & $-.25 * * *$ & $-.297 * * *$ & $-.298 * * *$ \\
\hline Middle North Island & & -0.0238 & -0.0238 & -0.0152 & -0.0102 & -0.0559 & -0.0533 & -0.0396 & -0.093 & -0.0751 \\
\hline Wellington & & 0.0755 & 0.0755 & $0.0914^{*}$ & 0.0779 & 0.078 & 0.0523 & -0.0033 & -0.0483 & -0.0411 \\
\hline Rest of South Island & & -0.158 & -0.158 & -0.149 & -0.166 & -.231 & $-.25^{*}$ & -0.197 & -.258 & -0.248 \\
\hline Canterbury & & -0.0359 & -0.0359 & -0.0302 & -0.0615 & -0.0564 & -0.0906 & -0.0839 & $-0.179 *$ & -0.166 \\
\hline \multirow{2}{*}{\multicolumn{11}{|c|}{ Sex (Base Female) }} \\
\hline & & & & & & & & & & \\
\hline Male & & & -0.00096 & -0.00714 & -0.00538 & -0.0638 & $-.111^{*}$ & $-.166^{* *}$ & $-.158 *$ & $-.179 *$ \\
\hline & & & & & & & & & & \\
\hline \multicolumn{11}{|l|}{ Age Groups } \\
\hline $15-19$ & & & & $.345^{*}$ & $.336^{*}$ & $.756 * * *$ & $.878 * * *$ & $.941 * * *$ & $.926 * * *$ & $.842 * * *$ \\
\hline $20-24$ & & & & -0.0983 & -0.109 & 0.158 & .212 & $.359^{*}$ & $.355^{*}$ & $.345 * *$ \\
\hline $25-34$ & & & & -0.0174 & -0.0203 & 0.0556 & 0.0787 & 0.116 & 0.114 & .127 \\
\hline \multicolumn{11}{|l|}{ Base: $35-44$} \\
\hline $45-54$ & & & & -0.122 & -0.113 & -0.0444 & -0.0391 & -0.00104 & -0.00037 & .0118 \\
\hline $55-64$ & & & & 0.138 & 0.147 & $0.17 *$ & 0.168 & $.217^{*}$ & $.203 *$ & $.199 *$ \\
\hline $65+$ & & & & $.604 * * *$ & $.611 * * *$ & $.690 * * *$ & $.811 * * *$ & $.87 * * *$ & $.836 * * *$ & $.766 * * *$ \\
\hline \multirow[t]{2}{*}{ New Zealand European } & & & & & .153 & 0.0839 & 0.0356 & -0.0261 & -0.074 & -.102 \\
\hline & & & & & & & & & & \\
\hline \multirow[t]{2}{*}{ Partnered } & & & & & & $.679 * * *$ & $.604 * * *$ & $.573 * * *$ & $.555^{* * *}$ & $.540 * * *$ \\
\hline & & & & & & & & & & \\
\hline \multicolumn{11}{|l|}{$\begin{array}{r}\text { Employment Status } \\
\text { (Base Employed) }\end{array}$} \\
\hline Unemployed & & & & & & & $-.836^{* * *}$ & $-.697 * *$ & $-.642 * *$ & $-.591 * * *$ \\
\hline Not in Labour Force & & & & & & & $-.349 * *$ & -.203 & -.182 & -0.165 \\
\hline D. & & & & & & & & & & \\
\hline Personal Income & & & & & & & & & & \\
\hline
\end{tabular}




\begin{tabular}{|c|c|c|c|c|c|c|c|c|c|c|}
\hline Loss/Zero & & & & & & & & 0.0317 & 0.0125 & .00728 \\
\hline$\$ 1-\$ 10,000$ & & & & & & & & -0.182 & -0.162 & -0.186 \\
\hline$\$ 10,001-\$ 20,000$ & & & & & & & & $-.261 *$ & $-.234^{*}$ & $-0.251^{*}$ \\
\hline$\$ 20,001-\$ 30,000$ & & & & & & & & -0.111 & -0.107 & -0.0985 \\
\hline \multicolumn{11}{|l|}{ Base: $\$ 30,001-\$ 40,000$} \\
\hline$\$ 40,001-\$ 50,000$ & & & & & & & & 0.0858 & 0.0843 & .0753 \\
\hline$\$ 50,001-\$ 70,000$ & & & & & & & & $0.192 *$ & 0.161 & .130 \\
\hline$\$ 70,001$ and above & & & & & & & & $.383 * *$ & $.299 *$ & .261 \\
\hline & & & & & & & & & & \\
\hline NZ Deprivation Index & & & & & & & & & $.515^{* * *}$ & $.513 * * *$ \\
\hline 2 & & & & & & & & & $.341 * *$ & $.323 *$ \\
\hline 3 & & & & & & & & & 0.253 & .268 \\
\hline 4 & & & & & & & & & 0.116 & .0901 \\
\hline \multicolumn{11}{|l|}{ Base: 5} \\
\hline 6 & & & & & & & & & 0.146 & .182 \\
\hline 7 & & & & & & & & & 0.0637 & .0824 \\
\hline 8 & & & & & & & & & -0.0294 & -0.0219 \\
\hline (High Dep) 10 & & & & & & & & & 0.0278 & .0477 \\
\hline & & & & & & & & & & \\
\hline \multicolumn{11}{|l|}{$\begin{array}{l}\text { Smoking Status (Base } \\
\text { Never Regular Smoker) }\end{array}$} \\
\hline Ex-Smoker & & & & & & & & & & -0.0934 \\
\hline Regular Smoker & & & & & & & & & & $-.218^{*}$ \\
\hline Constant & $9.85 * * *$ & $10 * * *$ & $10 * * *$ & $9.92 * * *$ & $9.92 * * *$ & $9.1 * * *$ & $9.26 * * *$ & $9.31 * * *$ & $9.25 * * *$ & $9.42 * * *$ \\
\hline $\mathrm{N}$ & 4971 & 4971 & 4971 & 4971 & 4971 & 4834 & 4803 & 4519 & 4446 & 4293 \\
\hline r2 & 0.00343 & 0.00703 & 0.00703 & 0.0225 & 0.0243 & 0.0527 & 0.0675 & 0.0737 & 0.0794 & .0798 \\
\hline r2_a & 0.00323 & 0.00583 & 0.00563 & 0.02 & 0.0215 & 0.0498 & 0.0642 & 0.0688 & 0.0725 & .0722 \\
\hline rmse & 1.79 & 1.79 & 1.79 & 1.77 & 1.77 & 1.73 & 1.72 & 1.69 & 1.69 & 1.69 \\
\hline 11 & -9947 & -9938 & -9938 & -9899 & -9894 & -9513 & -9408 & -8774 & -8620 & -8321 \\
\hline
\end{tabular}


In interpreting Table 6.2 above, I start with the effect of introducing controls on the relationship between life satisfaction and standard deviation life satisfaction for Māori. The effect in Model 1 is identical to that in the weighted ecological regression, with a hypothetical one unit increase in the standard deviation of life satisfaction (on the 11-point scale) resulting in a -1.181 decrease in individual life satisfaction ( $a=9.489$, $b=-1.181, S E=0.290, p<0.001)$. Introducing a control for the respondent's region increases the coefficient for a one unit increase to $-1.253(a=10.037, b=-1.253$, $S E=0.184, p<0.001)$.

The primary slope coefficient remains the same when sex is introduced in Model 3 and is lowered slightly when age groups are controlled for in Model 4. Controlling for identifying as New Zealand European raises the coefficient again slightly to its strongest in all the Models at $-1.282(a=9.917, b=-1.282, S E=0.176, p<0.001)$. Introducing partnership status drops the coefficient by a considerable margin to -1.038 ( $a=9.097, b=-1.038, S E=0.170, p<0.001)$, and introducing employment status results in a smaller drop, while introducing income group controls raises the coefficient slightly. Controlling for the respondent's New Zealand Deprivation Index rating drops the coefficient of the slope slightly and reduces the statistical significance $(p<0.01)$, while controlling for smoking status (an objective measure of health) raises the coefficient.

In Model 10, a hypothetical one unit increase in life satisfaction inequality in an area results in a -1.028 drop in individual life satisfaction $(a=9.419, b=-1.028$, $S E=0.310, p<0.01)$. In Model 10, the $\mathrm{R}^{2}$ is 0.080 , and the $\mathrm{N}$ has dropped to 4,293 from 4,971 in Model 1. Given that the slope coefficient only drops from -1.181 in Model 1 to -1.028 in Model 10, it seems that non-subjective controls account for very little of the sensitivity of Māori to wellbeing inequality among other Māori in their area.

Model 11 introduces subjective health, the first of the subjective controls. As expected when introducing a highly correlated (likely endogenous) control, the slope coefficient for standard deviation life satisfaction drops substantially $(a=8.837, b=$ $0.868, S E=0.259, p<0.01)$. Controlling for social isolation in the final model, Model 12, an increase in standard deviation life satisfaction still has a large and statistically significant effect on individual life satisfaction $(a=8.142, b=-0.773, S E=0.239, p<0.01)$. Even after introducing subjective controls on life satisfaction, it appears as though The Primary Hypothesis is supported at the individual level for Māori in relation to other Māori when drawing on Te Kupenga. 
It is worth commenting on the regional geography of the levels of wellbeing for Māori. When region is introduced as a control, living in Auckland is significantly negative for individual life satisfaction at $-0.255(p<0.001)$, compared to living elsewhere in the north of the North Island, consistent with the other surveys. The negative effect of living in Auckland on Māori wellbeing grows stronger as controls are introduced and remains significant. As noted by Morrison and Weckroth (2017) finding SWB to be significantly lower in the largest metropolitan region of a developed country such as New Zealand is common. The other regions are only intermittently significant throughout the models. However, note that when compared to living in the north of the North Island (excluding Auckland) the coefficient of the effect of living in all regions is negative, suggesting that Māori living in Northland may be more satisfied than Māori living elsewhere.

Finally, I draw attention to Figure 6.4, which provides a plot of the coefficients for the regression in Model 10 of Table 6.2. Figure 6.4 allows the easy visual comparison of the effects of controls and standard deviation life satisfaction on individual life satisfaction.

Figure 6.4: Coefficient plot of standard deviation life satisfaction and controls on individual life satisfaction

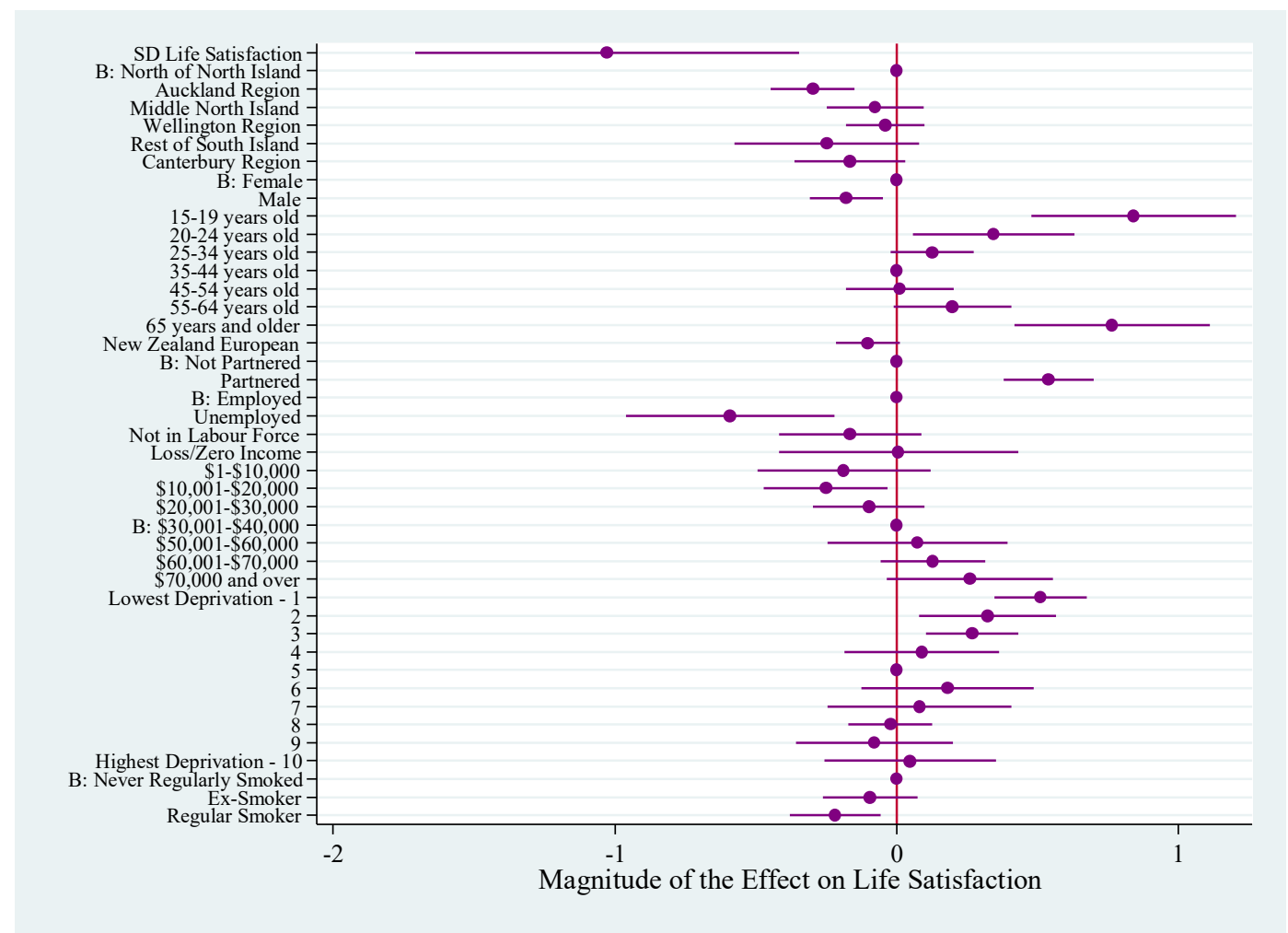

Figure 6.4: Te Kupenga, 2013, New Zealand 
It is clear from Figure 6.4 that a one unit increase in the standard deviation of local life satisfaction likely has the largest effect on individual Māori life satisfaction. However, it is possible that other effects like being unemployed could have an equivalent effect on wellbeing due to the overlapping confidence intervals. It is also possible I would find a clearer distinction between the effects if I were drawing on a sample with more effective geographic indicators for measuring life satisfaction inequality (like those used in the QoLS). Regardless, if a one unit increase in life satisfaction inequality were ever to occur, it would be at least as negative for life satisfaction as unemployment (in contrast to employment), one of the strongest predictors of poor wellbeing (Layard et al., 2012; Winkelmann, 2009)

Taken together, the evidence from the analysis of Te Kupenga supports The Primary Hypothesis. Mean Māori life satisfaction in an area is negatively associated with standard deviation Māori life satisfaction in that area when the population size of the area is accounted for. Models at the individual level indicate that increasing local life satisfaction inequality still brings a decrease in levels of life satisfaction after controls are accounted for. In this thesis, I have consistently found evidence of a negative relationship between life satisfaction and life satisfaction inequality at the subnational level. However, the analysis in Chapter 5 did not provide complete clarity regarding what underlies the relationship between wellbeing and local wellbeing inequality. The following sections will therefore reassess The Fairness Hypothesis and The Altruism Hypothesis using Te Kupenga.

\section{2: The Fairness Hypothesis}

Trust and perceptions of fairness are interconnected. In their analysis of the drivers of SWB sensitivity to income inequality, Oishi and colleagues (2011) indicate that both high perceptions of fairness and high trust in others protect the happiness of people from decline in years where there is greater income inequality. However, Delhey and Dragolov (2014) argue that these two measures are actually one and the same when using subjective opinions of others fairness:

"Oishi et al. aim at the perceived (un)fairness of the income distribution as a systemic feature, the concrete item used taps into individual honesty ('people try to be fair'), hence there is a mismatch between concept and operationalization. Further, many researchers use this 'fairness' item in conjunction with trust in most people for measuring one single 
underlying construct, dubbed either 'faith in people' (Rosenberg, 1957) or 'trust' (Reeskens and Hooghe, 2008). Hence, one can have doubts as to whether Oishi et al. have actually uncovered two different mediators, or just one." (p. 152, italics original)

As such, I use general trust in others separately from the average perceptions of fairness in organisations as both representative of perceiving the fair treatment of others more broadly. I expect that Māori who perceive society to be unfair will exhibit a greater sensitivity to wellbeing inequality than Māori who perceive a fair society, like individuals in the NZGSS. However, the magnitude of this relationship may be stronger for Māori, given the generally more community-oriented nature of their wellbeing (Cram, 2014).

Perceptions of fairness is measured in six separate questions on 11-point Likert scales. Like in the NZGSS, these questions were combined to make a measure of an individual's average perceptions of fairness. Both average perceptions of fairness and trust (also an 11-point Likert scale) in Te Kupenga were transformed into a binary variable using the same method detailed in the NZGSS. Those who perceive society to be fair or who believe most people can be trusted are assigned a value of ' 1 ' on each of the variables, while those who think the opposite are assigned a value of ' 0 '.

Figure 6.5 compares predicted sensitivity to life satisfaction inequality for those who perceive society to be fair in contrast with those who do not. No controls are included in Figure 6.5. As in Chapter 5, the red thick dashed line and shaded area represents those who do perceive society treats people fairly, while the blue thin solid line and shaded area represents those who perceive that society does not treat people fairly.

Figure 6.5 offers clear support for The Fairness Hypothesis. The life satisfaction of those who perceive society to be fair is less sensitive to life satisfaction inequality than those who perceive that society treats people unfairly. With every unit increase in standard deviation life satisfaction, people who perceive a fair society are 1.752 more satisfied with life than those who perceive an unfair society $(a=11.154, b=1.752$, $S E=0.413, p<0.01)$. The $\mathrm{R}^{2}$ of this regression is 0.037 . 
While perceiving society to be fair accounts for a much lower sensitivity to life satisfaction inequality, does not entirely cancel out the negative effects of it in Figure 6.5 , as the coefficient for life satisfaction inequality alone is still higher at -2.112 $(a=11.154, b=-2.112, S E=0.385, p<0.001)$.

\section{Figure 6.5: Predicted individual life satisfaction by standard deviation life satisfaction by perceptions of fairness, no controls}

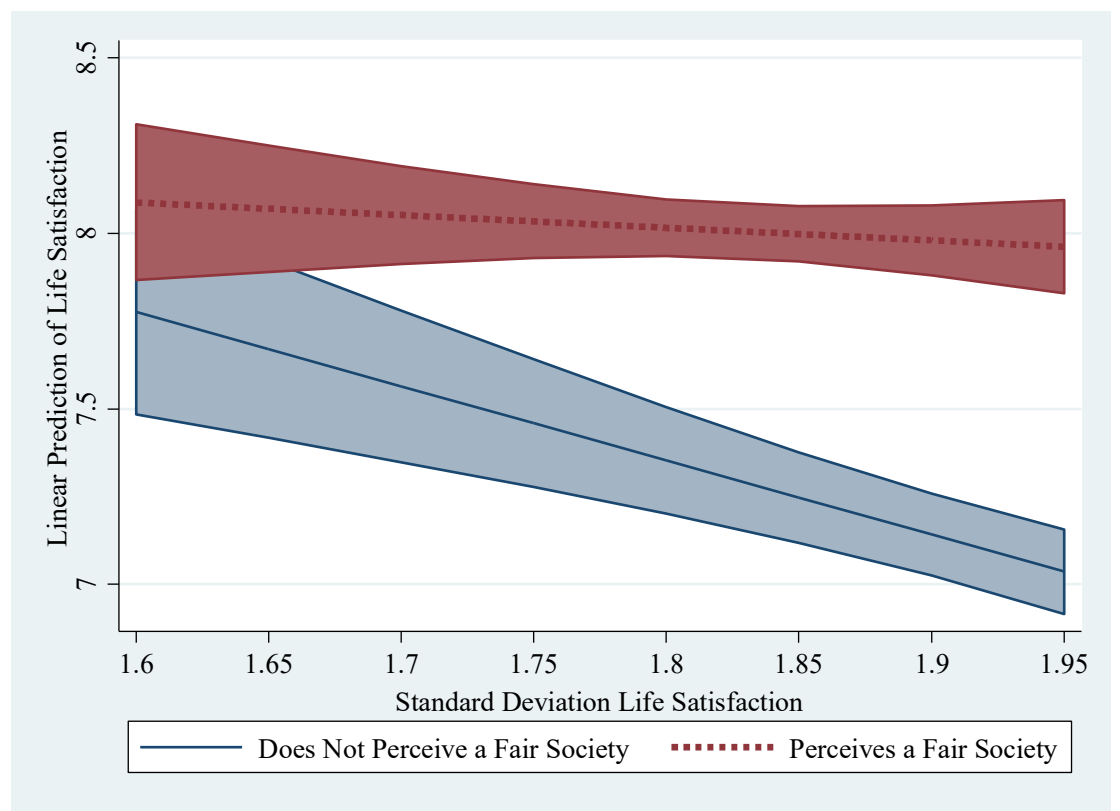

Figure 6.5: Te Kupenga, 2013, New Zealand

This first result is encouraging for The Fairness Hypothesis. However, another indicator of perceptions of fairness is available in Te Kupenga, and should be tested. Figure 6.6 displays the predicted life satisfaction of people who agree that others can generally be trusted (thick red dashed line) in contrast to those who do not (blue solid line. According to The Fairness Hypothesis, I expect that those who do agree that others can be trusted are less sensitive to life satisfaction inequality than those who do not agree that others can be trusted.

The predicted values of life satisfaction in Figure 6.6 provide further support for The Fairness Hypothesis, although the magnitude and significance of the effect is slightly lower than that demonstrated in Figure 6.5. For every unit increase in standard deviation life satisfaction, individual life satisfaction falls by $-1.861(a=10.728, b=$ $1.861, S E=0.664, p<0.05)$ for the group as a whole, but is $1.396(a=10.728, b=1.396$, $S E=0.563, p<0.05)$ higher among those who agree that others can be trusted compared to those who do not. The $\mathrm{R}^{2}$ of this regression is 0.030 . 
$\mathbf{1 0 5} \mid \mathrm{P}$ a g e

Figure 6.6: Predicted individual life satisfaction by standard deviation life satisfaction by trust in others, no controls

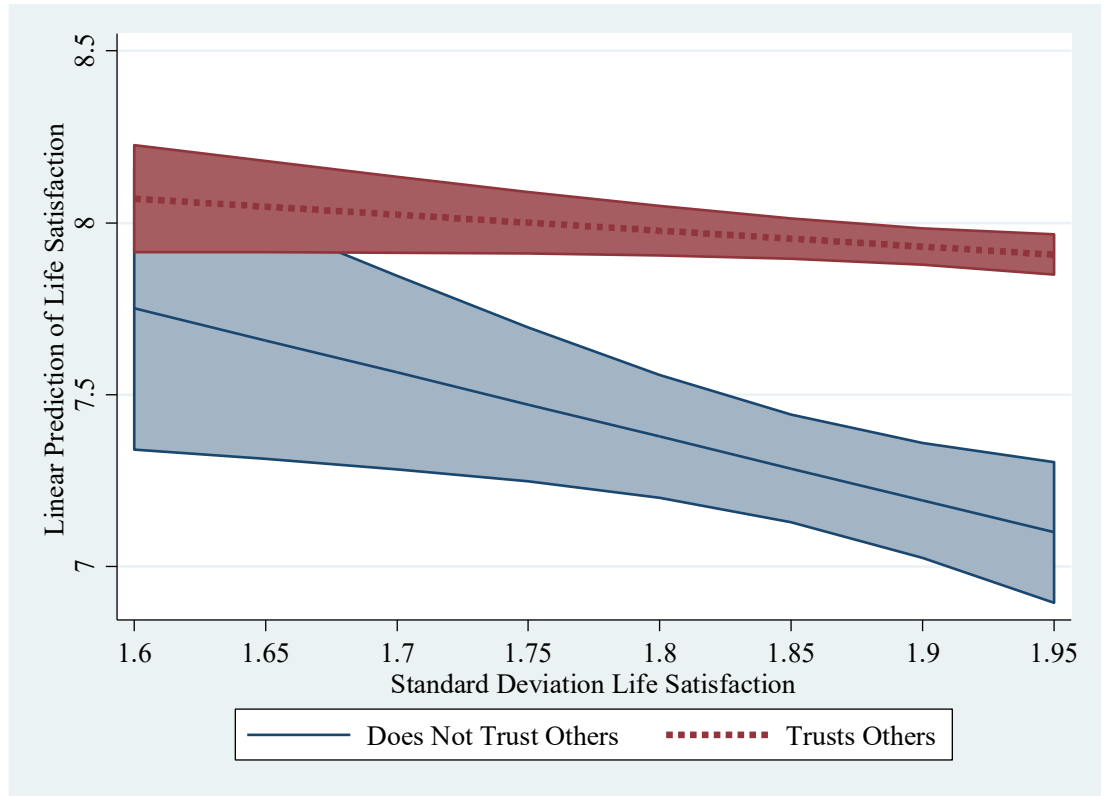

Figure 6.6: Te Kupenga, 2013, New Zealand

The regressions for Figure 6.5 and 6.6 both offer strong support for The Fairness Hypothesis. At this stage, it seems as though Delhey and Dragolov (2014) may have been correct in claiming that perceptions of fairness and beliefs that others can be trusted are actually measures of the same underlying values, as they interact similarly with sensitivity to wellbeing inequality here.

However, until I control for other factors, I cannot be certain that the Te Kupenga data really supports the Fairness Hypothesis. I run the 12 models from The Primary Hypothesis using the interaction of the fairness perception binary and standard deviation life satisfaction as the base model $^{29}$.

In each of the 12 Models, those who perceive society to be fair are less sensitive to life satisfaction inequality than those who do not. In Model 10 when smoking status is introduced, the coefficient of the interaction has fallen to only 1.635 ( $a=10.613$, $b=1.635, S E=0.379, p<0.01$ ) from 1.752 in Model 1. It appears that introducing nonsubjective controls has little impact on the effect of fairness perceptions on sensitivity to life satisfaction inequality. Introducing the subjective controls lowers the coefficient to $1.252(a=9.160, b=1.252, S E=0.351, p<0.01)$ in Model 12. In both instances the

\footnotetext{
${ }^{29}$ Due to space constraints the estimates tables are not presented here.
} 
effect of the interaction is not enough to cancel out the negative impacts of life satisfaction inequality entirely, and in both cases the effect of life satisfaction inequality is still significantly negative ( $p<0.001$ for Model 10 and $p<0.01$ for Model 12).

I also run the 12 Models for the interaction between the trust binary and standard deviation life satisfaction. The interaction between the two is significant until Model 8 , when income is introduced. After Model 8, people who believe most others can be trusted are still less sensitive to life satisfaction inequality, but this sensitivity is not significant $(p=0.106)$. The introduction of additional controls only weakens the significance further.

Why might economic indicators weaken the effects of trust? Model 9 of Table 6.2 indicates that people living in the least deprived areas of New Zealand are significantly more satisfied than people in other areas. Chapter 5 also indicated that deprivation may play a part in the relationship between perceptions of fairness and sensitivity to wellbeing inequality. As such, I ran the regression in Model 9 separately for the least deprived groups in New Zealand (1 on the New Zealand Deprivation Index) and for all the other groups (2-10). Both groups still showed a non-significant interaction. Evidently, once you control for regional, demographic, achievement and subjective controls, an individual's belief that others can be trusted does not influence their sensitivity to life satisfaction inequality.

Taken together, the results in this section still constitute support for The Fairness Hypothesis. Comparing people based on their perceptions of fairness in society using a more comprehensive measure than that used in Chapter 5 reveals that those who believe society treats people fairly are less sensitive to wellbeing inequality, even after a full set of controls are introduced. Testing the sensitivity of those who trust others compared to those who do not reveals a similar relationship without controls. However, once controls are introduced, the differences according to beliefs that others can be trusted disappear.

Re-assessing Delhey and Dragolov's (2014) claim, perceptions of fairness and belief that others can be trusted do appear to be distinct. While they may both account for similar underlying values, direct measures of fairness perceptions are much more significant and robust explanatory mechanisms of sensitivity to wellbeing inequality than trust beliefs. Therefore, I am inclined to support Oishi and colleagues (2011) in 
identifying fairness perceptions and trust in others as two separate drivers of sensitivity to inequality.

The following section will retest The Altruism Hypothesis using data from Te Kupenga, and will be the final analysis section of my thesis.

\section{3: The Altruism Hypothesis}

In Chapter 5, I found no support for the hypothesis that altruistic individuals are more sensitive to wellbeing inequality when drawing from NZGSS data. As the tests using sex and age group were ineffective, those variables will not be used. No specific volunteering variable is available in Te Kupenga for analysis. However, there are eight binary 'unpaid work' variables in Te Kupenga, which may represent altruistic tendencies. While volunteering has no significant effect on sensitivity to wellbeing inequality in the NZGSS, testing the effect of eight separate variables allows for a more robust test of The Altruism Hypothesis.

Again, I expect that differences due to culture will likely be present. Robinson and Williams (2001) discuss Māori perspectives on volunteering and giving in contrast with non-Māori perspectives. They highlight that Māori traditionally see providing help to others as a cultural obligation, emphasising collective responsibility to care for others. They explain:

"[Māori] are driven more by manaaki, tautoko etc., concepts underpinned by collective responsibility (beneath these). These values and the practice of them have nothing to do with how much you get paid, what resources/skills you have, but about your duty and your obligation, though your resources might get pulled into that." (Robinson \& Williams, 2001:65)

As such, there are three possible outcomes from analysis of The Altruism Hypothesis when using a dataset of Māori respondents. One possibility is in line with the hypothesis itself: that Māori who display altruistic values will be more sensitive to wellbeing inequality than those who do not. However, given Robinson and Williams' (2001) argument that Māori conceptualise volunteering in a very different way (with altruistic values not necessarily underlying that conceptualisation), a second expectation is that altruism as measured by voluntary work has no effect on sensitivity to life satisfaction. A third possibility is that Māori who do participate in unpaid work are 
actually less sensitive to wellbeing inequality than others: this result could be explained either by the protective role that volunteering plays in general (Binder, 2015) or by the culturally-specific values underlying volunteer work for Māori (Robinson \& Williams, 2001).

Eight variables identify whether individuals have participated in a variety of activities helping others without pay in the previous four weeks. Of the eight variables, two have a significant effect on the sensitivity of individuals to life satisfaction: unpaid work for 'a school, church, sports club or other group or organisation', and unpaid work 'cooking, cleaning, gardening, doing repairs, or any other housework for someone who doesn't live with you' ${ }^{30}$. The other six unpaid work variables have no significant effect on the relationship between life satisfaction and life satisfaction inequality for Māori.

Figure 6.7 plots the predicted effect of providing unpaid help to groups or organisations in an individual's community on sensitivity to life satisfaction inequality. The red dashed line on top represents those who have provided help to community groups or organisations, while the thin blue line on the bottom represents those who have not provided help to community groups or organisations.

Figure 6.7: Predicted individual life satisfaction by standard deviation life satisfaction by community help provided, no controls

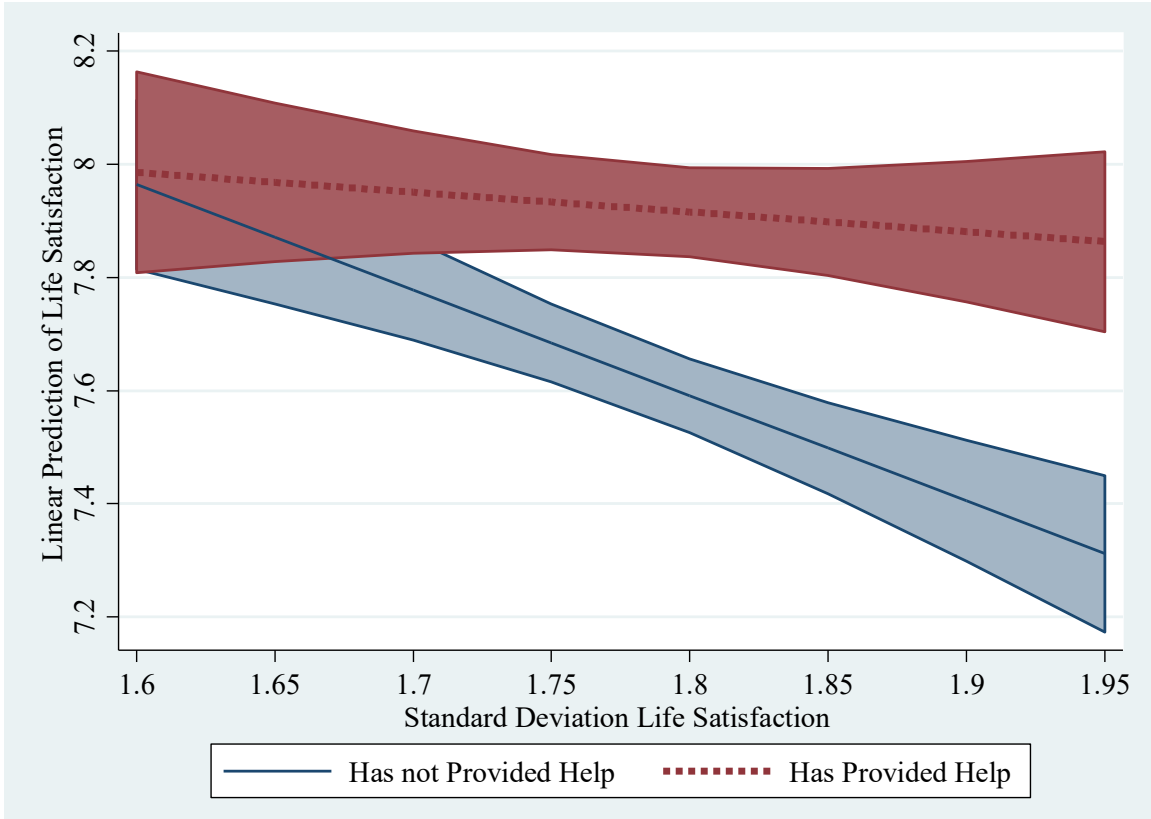

Figure 6.7: Te Kupenga, 2013, New Zealand

${ }^{30}$ The eight variables are described in full in Appendix A, but are left out here owing to their length. 
Rather than offering support for The Altruism Hypothesis, the regression in Figure 6.7 indicates I should reject it. For every one unit increase in standard deviation life satisfaction, those who help groups in their community are 1.514 points more satisfied than those who do not help groups in their community, while for every 0.5 unit increase the effect is 0.757 , and for a 0.2 unit increase it is 0.303 ( $a=10.945, b=1.514$, $S E=0.574, p<0.01)$. The $\mathrm{R}^{2}$ of this equation is 0.012 . If helping others in your community is indeed indicative of altruism, then altruistic people are actually less sensitive to wellbeing inequality than non-altruistic people, having a similar effect to perceiving a fair society. However, as noted, altruistic values may not underlie unpaid work for Māori (Robinson \& Williams, 2001), or even for the general population (Becchetti et al., 2017).

I turn to the other altruism variable which significantly effects sensitivity to wellbeing inequality to see if the effect is consistent: unpaid housework for other people. Figure 6.8 provides a plot of the predicted effect of life satisfaction inequality on individual life satisfaction for those who provide unpaid housework for others and for those who do not. The same colour scheme used in Figure 6.7 is also used in Figure 6.8 .

Figure 6.8: Predicted individual life satisfaction by standard deviation life satisfaction by housework help provided to those outside of the household, no controls

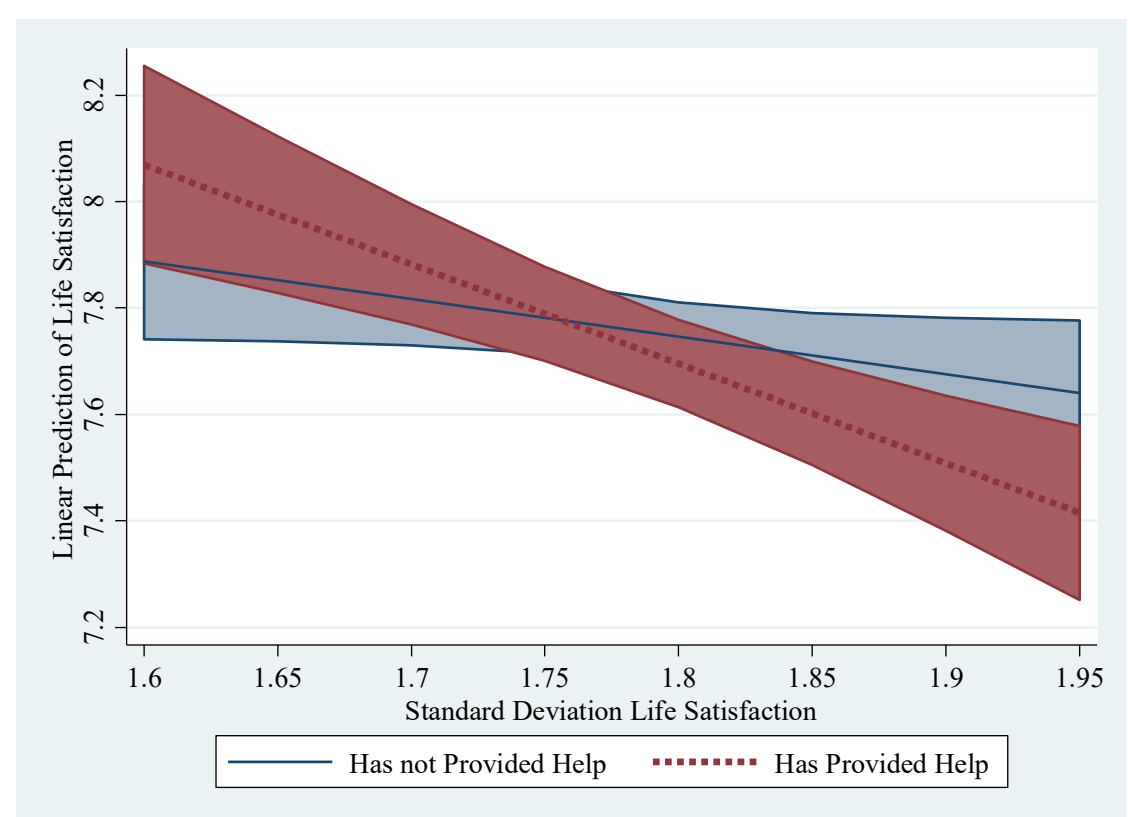


The results in this instance offer some support for The Altruism Hypothesis. For every unit increase in life satisfaction inequality, individual life satisfaction decreases by -1.164 more for those who do provide unpaid housework assistance ( $a=9.015, b=-$ 1.164, $S E=0.582, p<0.05)$. However, the $95 \%$ confidence intervals of the two groups largely overlap, suggesting that evidence for a significant effect might be weak. The $\mathrm{R}^{2}$ for this regression is also low relative to others, at only 0.004 .

To test for the effect of controls I ran the same 12 models used throughout this chapter. ${ }^{31}$ First I test for the effect of controls on the relationship in Figure 6.7, by using the interaction between standard deviation life satisfaction and unpaid help in groups such as school and churches as the base model. The difference between those who provide unpaid help in community groups and those who do not actually grows wider with controls, and the effect is statistically significant in each model. In Model 10 when smoking status is introduced, those who do provide unpaid help in their community are 2.003 more satisfied with their lives per unit increase in standard deviation life satisfaction than those who do not help out in their communities ( $a=10.768, b=2.003$, $S E=0.402, p<0.001)$. The size of this effect in Model 10 is substantively large, and suggests that providing unpaid help in the community plays a strong protective role in the individual wellbeing of Māori. Indeed, the coefficient more than compensates for the negative effect of -1.853 for standard deviation life satisfaction overall in Model 10 $(a=10.768, b=-1.853, S E=0.300, p<0.001) . \quad$ The $\mathrm{R}^{2}$ in Model 10 is 0.093 .

Introducing the subjective controls lowers the coefficient of the slope to 1.519 in Model $12(a=9.234, b=1.519, S E=0.405, p<0.01)$. However, this final effect is still stronger in magnitude than in Model 1 at 1.514, and overcompensates for the effect of just standard deviation life satisfaction in Model 12 at $-1.429(a=9.234, b=-1.429$, $S E=0.284, p<0.001)$. The $\mathrm{R}^{2}$ for Model 12 is 0.192 .

The results from this succession of models indicates that providing unpaid help to or through a group or organisation (such as a school, church, or sports club) plays a strong protective role regarding wellbeing inequality. Such a finding is in keeping with Binder (2015), who indicates that volunteering protects the subjective wellbeing of volunteers in the face of otherwise adverse conditions. In Models 10 and 12 with a large number of controls, individual wellbeing even rises in the presence of greater

\footnotetext{
${ }^{31}$ Due to space constraints the estimates tables are not presented here.
} 
$111 \mid \mathrm{P}$ a g e

inequality for those who provide unpaid help. This may be a result of these helpful individuals sensing greater inequality, but also feeling that they are fulfilling their cultural obligation to redress this inequality (Robinson \& Williams, 2001). Further research is necessary to test this speculation

I also test the effect of providing unpaid housework to others in the same series of 12 Models, to see whether the relationship identified in Figure 6.8 persists. The significance of the difference between those who do unpaid housework for others and those who do not disappears with the introduction of regional controls in Model 2 $(p=0.133)$. The effect does not return for any of the Models after it.

My analysis finds no evidence to support The Altruism Hypothesis. Six of the eight variables theoretically connected to altruism in Te Kupenga have no significant effect on the relationship between wellbeing and wellbeing inequality. Only providing unpaid help in community groups or organisations has a significant effect on sensitivity to wellbeing inequality after introducing controls. The substantive effect of this variable is large, but runs counter to The Altruism Hypothesis. All evidence in Te Kupenga indicates that The Altruism Hypothesis should be rejected. However, the finding that providing help to community groups serves a protective function for Māori regarding wellbeing inequality is novel, and warrants further investigation in future research.

\section{4: Summary}

In spite of the fact that Te Kupenga contains data exclusively on Māori, the overall findings of this chapter are remarkably similar to those for the general population in Chapters 4 and 5, suggesting that sensitivity to wellbeing inequality is common across New Zealand cultures. The Primary Hypothesis has been confirmed once again at the ecological and the individual level using Te Kupenga, indicating that the negative relationship between wellbeing and wellbeing inequality exists for Māori relative to Māori as well as for the general population relative to the general population.

I analysed The Fairness Hypothesis using two strong measures of perceptions of fairness from Te Kupenga: perceptions that society treats people fairly, and belief that others can be trusted. I found support for The Fairness Hypothesis using both measures, but found that this support was only robust after introducing a full set of controls for the more direct measure of perceptions of fairness. That I found a difference between the effects of 'perceptions of fairness' and 'trust in others' on sensitivity to wellbeing inequality adds credence to Oishi and colleagues (2011) argument that the 
measures account for distinct (albeit related) values, in contrast to Delhey and Dragolov's (2014) claims that they are the same.

Finally, I re-assessed The Altruism Hypothesis in this chapter using eight variables regarding participation in unpaid work. Based on the analysis, The Altruism Hypothesis should be rejected. Seven of the eight variables have no significant effect on sensitivity to wellbeing inequality after controls are introduced. Doing unpaid work in community groups like schools and churches does impact individual sensitivity to wellbeing inequality, but in the opposite way from my predictions: those who perform altruistic activities are actually less sensitive to wellbeing inequality than others. It is worth bearing in mind the role that the culturally specific nature of Māori participation in voluntary work may play in this result (Robinson \& Williams, 2001), as well as the protective role that volunteering plays for wellbeing more broadly (Binder, 2015).

In the following chapter, I will discuss the results my conclusions for each of the five hypotheses. I will also discuss the potential policy implications of these conclusions. Finally, I will highlight the limitations and constraints of my research, before discussing the areas appropriate for future research that my own analysis has uncovered. 


\section{Chapter 7: Conclusion}

For over 40 years researchers have been trying to determine why people's wellbeing varies. Recent work points to a robust negative relationship between the average subjective wellbeing of countries and the distribution of subjective wellbeing within them (Bolle et al., 2009; Delhey, 2004; Fahey \& Smyth, 2004; Goff et al., 2016; Ott, 2005; Quick, 2015). The fact that our wellbeing falls as wellbeing distributions widen suggests we care about inequalities in the lives of others. However, to date little attention has been paid to this relationship at the sub-national level within regions and cities (Tesfazghi et al., 2010). The contribution of my thesis is to take the next step in research on variations in wellbeing by showing that the same relationship continues to hold within countries. My New Zealand evidence not only confirms the generality of the relationship identified at the inter-country level, it also has potentially profound implications for wellbeing policy at the national and local level.

My enquiry into the role of fairness suggests that we may be protected from the negative impacts of inequality in wellbeing around us by having a positive (and perhaps unrealistic) outlook on society. I have systematically tested the effect of individual perceptions of fairness in society, and have demonstrated that those who believe individuals are treated fairly are less sensitive to wellbeing inequality than those who are more sceptical. I have also tested the impact of altruistic tendencies, but have found little effect. In the instance where an effect was found, Māori with altruistic tendencies were less sensitive to inequalities in wellbeing than Māori without them.

This concluding chapter provides synthesis of each of the following hypotheses: The Primary Hypothesis, The Parochialism Hypothesis, The Fairness Hypothesis, and The Altruism Hypothesis.

Section 7.2 considers possible policy implications of the findings. The chapter ends in section 7.3 with a discussion of the limitations of the current research and recommendations for future research pursuits in the SWB inequality field.

\section{1: Were the Hypotheses Supported?}

I drew on responses to similar life satisfaction questions from three surveys: the NZGSS, the QoLS and Te Kupenga, which together covered a population of 41,985 individuals spread over five different years. The first hypothesis I tested was that people's wellbeing would decline with the increasing inequality in wellbeing around 
them. A negative relationship between SWB and inequality in SWB was expected on the basis of previous research at the international level (Bolle et al., 2009; Delhey, 2004; Fahey \& Smyth, 2004; Goff et al., 2016; Ott, 2005). However, to date the theoretical framework and methodology has not been considered and applied at the sub-national level.

My research used sub-national data from the three surveys to examine the same relationship. In each survey a consistent negative relationship was found between life satisfaction and the dispersion in life satisfaction among sub-national spatial groups and individuals. Analysing Te Kupenga confirmed that the negative relationship between wellbeing and wellbeing inequality also exists for Māori, in spite of their different conceptualisation of wellbeing compared to non-Māori (Cram, 2014). The QoLS contained the greatest number of geographic indicators, and so produced the most robust regressions of life satisfaction and life satisfaction inequality. In both of the survey years I used, a hypothetical increase of one standard deviation in life satisfaction inequality was associated with an almost one unit decrease in life satisfaction on the 5point scale. The predicted impact was greater than that of being unemployed, one of the strongest predictors of poor wellbeing in the field (Dolan et al., 2008; Layard et al., 2012; Winkelmann, 2009). In the NZGSS and Te Kupenga surveys, the impact of a one unit increase in the standard deviation of life satisfaction was associated with an impact on life satisfaction that was at least of the equivalent magnitude experienced by the unemployed.

These empirical findings suggest that the wellbeing inequality of those around us is one of the strongest predictors of our own wellbeing. As such, my findings confirm that the lack of attention to wellbeing inequality internationally needs to be rectified (Helliwell et al., 2016).

In earlier research, doubt was cast on the statistical validity of the wellbeing and wellbeing inequality relationship due to the ceiling (or 'bounded') properties of SWB scales (Delhey \& Kohler, 2011). These have now been largely dismissed as important considerations (Goff et al., 2016), and my own tests of the role of explanatory mechanisms help confirm the presence of substantive reasons for the negative relationship.

The first explanation I offered for sensitivity to local wellbeing was parochialism. Parochialism as a theory asserts that individuals are most sensitive and 
$\mathbf{1 1 5} \mid \mathrm{P}$ a g e

averse to inequality in smaller groups (Fehr et al., 2008). As such, I predicted that the wellbeing and wellbeing inequality relationship would be more negative at successively smaller scales.

The most robust survey with which to test this hypothesis is the QoLS, as it contains a high number of wards nested within six New Zealand cities. In contrast, the NZGSS and Te Kupenga both contain a relatively small number of geographic categories, and they are all quite broad as a result of the variables available. Bearing these restraints in mind, it is not encouraging that only mixed support for parochialism was found in the QoLS, while consistent support was found in the NZGSS. In the 2012 wave of the QoLS, the ecological regressions of life satisfaction and life satisfaction inequality were all greater in magnitude at the city level than at the ward level. However, the opposite was true for 2014, where the regressions at the city level were not even significant. In the NZGSS, regional effects were not significant in 2008, 2010 or 2012 ecological regressions, whereas they were significant and negative in the ecological regressions using more specific geographic indicators.

Taken together, these results offer tentative support for the idea that parochialism plays a part in individual sensitivity to local wellbeing inequality. Ideally, more geographic categories would have been available at all scales to test the effects of parochialism more thoroughly.

However, it is possible that the lack of parochialism recognised in the 2012 wave of the QoLS is representative of reality. Wards, while the most specific spatial unit used in this thesis, are not necessarily representative of an individual's in-group or community. People may consider themselves part of smaller neighbourhood community groups first and foremost, or perhaps as members of the city overall, potentially not considering their ward as an in-group at all. It is also worth bearing in mind an earlier point: that other in-groups may be more suitable for testing the influence of parochialism on sensitivity to wellbeing inequality. People may consider themselves members of religious or cultural groups more so than they consider themselves members of a spatial grouping, and so would likely be more sensitive to wellbeing inequality in those groups than in their local area. There is certainly potential for future research on how parochialism effects sensitivity to wellbeing inequality.

I also assessed perceptions of fairness as a potential explanation for sensitivity to wellbeing inequality. Although individual perceptions of fairness in society have not 
been used to explain sensitivity to inequalities in SWB, they have been used to explain sensitivity to income inequality (Delhey \& Dragolov, 2014; Katic \& Ingram, 2017; Oishi et al., 2011). In addition, one study used a similar measure (beliefs that income inequality is too high) to identify differences in sensitivity to wellbeing inequality (Goff et al., 2016). As such, the extent to which perceptions of fair treatment in society modify sensitivity to SWB inequality was tested in this thesis.

The hypothesis that those who perceive society to be fair are less sensitive to inequality in wellbeing has been supported by the New Zealand data. Life satisfaction data from both the NZGSS and Te Kupenga was used in order to address The Fairness Hypothesis, alongside a variety of variables measuring perceptions of fairness and trust in society. In the NZGSS, respondents were asked about their perceptions regarding how fairly people in New Zealand are treated by a variety of institutions and organisations. Averaging an individual's responses across these different organisations provided a composite variable representing their average beliefs that society is fair. Comparing those who do perceive society to be fair with those who do not, I found evidence that the former are significantly less sensitive to inequality in their area than the latter. Introducing controls completely diminished this effect in 2012 and slightly reduced the significance of it in 2010, but the effect remained strong in 2008 .

I speculated that increasing sensitivity to wellbeing inequality of those who perceive society to be fair is the result of changed macro conditions and a changing political climate. In 2008, Labour was reaching the end of a long stint in government, and demand for the labour force was still high. After a long period of attention to inequality under Labour, I suspect that individuals who perceived society as fair felt that the distribution of wellbeing was 'fair enough' and so had lower sensitivity to it. Indeed, research supports the idea that people favour greater inequality in periods with a Labour government, and favour less inequality with a National government (Morrison, 2015a). The convergence in sensitivity over time in 2010 and 2012 (i.e. those who perceive society to be fair get more sensitive to inequality) was likely due to the increase in more visible forms of inequality under the National government that came along with stark rises in the unemployment rate (among other macroeconomic trends).

In order to provide some test of this speculation, I compared the effects of fairness perceptions on sensitivity to wellbeing inequality between individuals who live in high deprivation and low deprivation areas (as defined by the New Zealand 
$117 \mid \mathrm{P}$ a g e

Deprivation Index). I anticipated that those who were in more deprived areas would be more aware of unequal distributions in society, and so would be more sensitive to them. However, in 2008, those in the highest deprivation areas of New Zealand (a 9 or 10 on the Index) were not sensitive to life satisfaction inequality. In contrast, it was the majority of individuals living in less deprived areas ( 0 to 8$)$ who demonstrated greater sensitivity to wellbeing inequality when they perceived an unfair society in 2008 . In 2010 there is little difference in sensitivity to wellbeing inequality between people in high and low deprivation areas, perhaps due to everyone suffering from recent marked rises in inequality.

However, in 2012 the relationships by deprivation are reversed in comparison to 2008, with perceptions of fairness in high deprivation areas playing a part in sensitivity to wellbeing inequality, while fairness perceptions have no effect for those in the less deprived majority. This result suggests that those living in high deprivation who also perceive society to be fair are the only ones significantly less sensitive to wellbeing inequality in 2012. Further research is needed to confirm the factors underlying these differences in the relationship over time.

The Te Kupenga survey tests support for The Fairness Hypothesis using two separate variables: perceptions of fairness in society, and belief that others can generally be trusted. Delhey and Dragolov (2014) argue that measures of trust in others and perceptions of fair treatment are both indicators of the same thing. Therefore, I compared the sensitivity to wellbeing inequality of those who have high trust with those who have low trust. In addition, I used a similar measure of average perceptions of societal fairness to that used in the NZGSS. My initial analysis of Te Kupenga suggested that those who have less trust in other people are more sensitive to SWB inequality, as are those with lower average perceptions of fairness.

However, after introducing a number of demographic (sex, age, and ethnic identity) and other (partnership, employment, income, deprivation, and smoking status) controls, the effect of trust in others on sensitivity to wellbeing inequality became statistically non-significant. By contrast, the effect of perceiving that society is fair remained strong and significant. Therefore, I disagree with Delhey and Dragolov's (2014) assertion that trust in others and perceptions of a fair society capture the same values. While they may be related, I agree with Oishi and colleagues (2011) in that the 
two measures are distinct. Regardless, an individual's perceptions of fairness $d o$ clearly play a part in their sensitivity to the dispersion in wellbeing around them.

Altruistic values have often been found to drive egalitarian preferences (Dawes et al., 2007; Dawes et al., 2012; Johnson et al., 2009). The Altruism Hypothesis predicts that those who have altruistic tendencies will be more inequality averse and hence more sensitive to local inequalities in wellbeing. Unfortunately, none of the surveys I use contain a direct measure of altruistic values, so I have had to use a number of proxies instead. As females have been found to be more altruistic in previous research (Rand et al., 2016; Simmons \& Emanuele, 2007), being female is used to test altruism. As the elderly are known to favour greater equality (Morrison, 2015b), age is also used as a proxy of altruism.

It is perhaps because of this lack of a direct altruism measure that The Altruism Hypothesis was largely unsupported. I found no statistically significant difference between male and female sensitivity to wellbeing inequality. Likewise, older age groups were never significantly more sensitive to wellbeing inequality.

The NZGSS also enabled me to analyse two behavioural variables as proxies for altruistic values in the form of volunteering and helping others. In Te Kupenga, eight variables recording participation in unpaid work were also available for analysis. However, previous research indicates that participation in behaviours such as volunteering may not always be motivated by altruistic behaviours (Becchetti et al., 2017). I am also aware that volunteering and similar activities may act to protect an individual's wellbeing in otherwise unsatisfactory conditions, such as inequality (Binder, 2015). Specifically within a Māori cultural context, unpaid volunteering has a very different conceptual underpinning compared to non-Māori, and does not necessarily represent altruism (Robinson \& Williams, 2001).

Perhaps because of these qualifications, no significant difference was found in the relationship between wellbeing and wellbeing inequality in the NZGSS. In Te Kupenga, helping out in community groups or organisations was associated with a lower sensitivity to wellbeing inequality. After introducing controls, the wellbeing of those who helped out in community groups actually rose slightly as inequality increased. The other seven unpaid work variables in Te Kupenga have no impact on sensitivities to SWB inequality after controls are introduced. 
This null result may be due to the protective role that Binder (2015) suggests volunteering serves for wellbeing. Robinson \& Williams (2001) indicate that helping others is perceived as a cultural obligation among Māori. That Māori who volunteer to help others get more satisfied as inequality increases may be a result feelings that they are fulfilling their cultural obligations. Regardless, my prediction that altruistic individuals would be more sensitive to wellbeing inequality is currently unsupported.

\section{2: Policy Implications}

The finding of a negative link between wellbeing inequality and both individual and average wellbeing at a sub-national level has a number of important implications. First and foremost, it indicates that policies aimed at increasing the wellbeing of a population will not necessarily succeed unless they also take the distribution of wellbeing into account.

My results imply that wellbeing inequality must be addressed alongside policies aiming to improve wellbeing overall. If a policy is put in place that does not consider its effects on the distribution of wellbeing, then we may find that any positive progress for one group is only offset by the increased wellbeing inequality around them. A corollary of such a focus is that we always ask 'who is likely to benefit most from a policy?' and 'who will be negatively impacted?'.

One of my findings that has a particularly important implication for policy is the magnitude of the wellbeing consequences of living in a more unequal area. I have showed that the effect of living in an area with one standard deviation lower life satisfaction is greater than the difference between someone who is employed full time and someone who is unemployed. Finding that the impacts of wellbeing inequality are so severe is evidence that the distribution of local wellbeing deserves immediate attention in policy. Measuring the wellbeing of citizens comprehensively and in diverse ways is an important start.

A purely statistical reading of my results suggests that without reducing wellbeing inequality through targeted intervention, we are unlikely to see as large an increase in individual or average wellbeing. Research at the international level has primarily recommended country-level changes to redress wellbeing inequality (Abdallah \& Quick, 2016; Ott 2005; 2011; Ovaska \& Takashima, 2010). For example, Ott (2011) argues that improving the technical quality of national governance will reduce wellbeing inequality. However, the presence of regional, city, and within city 
differences in wellbeing inequality suggests that policies at a local level are also likely to be essential.

In the introduction, I discussed the change in the Local Government Act (LGA) in 2012 under National. As a reminder, when the LGA was introduced in 2002, one of the two statutory purposes of local government was defined as: "to promote the social, economic, environmental, and cultural wellbeing of communities, in the present and in the future" (emphasis added). In 2012 National amended this purpose to: "to meet the current and future needs of communities for good quality local infrastructure, local public services, and performance of regulatory functions in a way that is most costeffective for households and businesses."

My research suggests that local government should have a greater influence on matters of wellbeing, not less. As such, I support the recently proposed amendment to the LGA: the "Local Government (Community Well-being) Amendment Bill". The bill proposes to reintroduce wellbeing to local government, explicitly changing the purpose of the LGA back to its 2002 condition (Parliamentary Counsel Office, 2018). The evidence I have assembled speaks strongly in favour of providing local government with a clear purpose and the requisite funding to address the underlying causes of local inequalities in SWB. Central and local government must pay greater attention to the distribution of wellbeing within localities if the wellbeing of all is to improve.

\section{3: Limitations and Recommendations for Future Research}

My thesis explored the relationship between SWB and SWB inequality in a complementary way to the literature comparing countries, and showed that such an approach is helpful for understanding variations in local wellbeing within countries as well. At the same time, my work also has limitations. These limitations revolve primarily around the analytical constraints imposed by the three datasets.

Perhaps the main limitation of this work has been the limited geographic variables available in the datasets. The QoLS permitted analysis within cities and wards, which allowed for the generation of standard deviation SWB variables within small scale geographic areas. However, even wards leave something to be desired, as they vary markedly in geographic size, and their boundaries can change subtly between years. Furthermore, the wards in the QoLS were confined largely to the main cities of New Zealand. 
The CURFs available for the NZGSS and Te Kupenga offer even less geographic resolution. The regions I used often spanned large portions of the country, and as such are not the most effective when trying to measure the sensitivity of an individual to the inequality in life satisfaction around them. While combining the region variables with the urban indicators helped somewhat, the geographic units used in the NZGSS and Te Kupenga still remained a considerable limitation of the current study.

A further limitation concerns the confidentialised nature of the NZGSS and Te Kupenga. Accessing the physical Statistics New Zealand data lab was a time consuming option that I was reluctant to take as part of a master's thesis, but as a result many of the variables in the datasets had to be used in either their collapsed form or were unavailable entirely. ${ }^{32}$ The opportunity to use more specific geographic indicators and to test certain hypotheses with more detailed variables would be possible with data lab access.

One limitation common to a great deal of social science literature is the crosssectional nature of my research. While both the QoLS and the NZGSS contain data on multiple years, neither of these datasets are longitudinal, and so are unable to hold personal characteristics stable between years. Furthermore, without longitudinal data I can never be certain of the causality in my results.

A positive result of the above limitations is that there are ample opportunities for future research into sub-national sensitivity to SWB inequality. The Parochialism Hypothesis could be re-tested using a diverse number of geographic scales, or even nongeographic groupings such as religious or ethnic groups. Further research could also investigate whether factors such as housing tenure or sense of belonging in a community may moderate the effect of parochialism on sensitivity to wellbeing inequality.

The addition of questions to New Zealand surveys on individual values such as altruism and perceptions of (or preferences for) fairness would be valuable for more specific testing of The Altruism Hypothesis and The Fairness Hypothesis. The findings of The Fairness Hypothesis are relatively robust, although the influence of changes in macro conditions and politics over time would be worth exploring alongside fairness perceptions using longitudinal data.

\footnotetext{
${ }^{32}$ Data lab access on campus was not available when I began this study.
} 
While using 5-point Likert scales to generate a measure of SWB inequality is acceptable, drawing on surveys with 10-point or 11-point scales is preferable, as it allows for greater variety in SWB. I would recommend that all New Zealand surveys move towards adopting an 11-point scale of life satisfaction.

In a more practical manner, future research should concern itself with understanding what factors are associated with being at the lower percentiles of the wellbeing distribution. Doing so will allow the development of interventions targeting unhappy and dissatisfied populations in particular. The marginal effect of living in areas with varying levels of wellbeing inequality could be tested more specifically.

\section{4: In Summary}

I have drawn from three large independent datasets to demonstrate that local levels of wellbeing inequality are negatively correlated with mean and individual wellbeing across regions in New Zealand. The statistical magnitude of this effect is strong and robust to numerous tests of the methodology conducted internationally.

I have investigated several psychological mechanisms underlying the negative link between wellbeing and wellbeing inequality. There is some evidence to support greater sensitivity to wellbeing inequality at successively smaller spatial scales as a result of parochialism. Testing for the effect of perceived fairness in society, I found that people who believe society treats everyone fairly are less sensitive to local wellbeing inequality. However, I was unable to find evidence using the proxies available to show that altruistic tendencies also drive sensitivity to wellbeing inequality in New Zealand.

I believe this is the first piece of research to extensively examine the subnational relationship between wellbeing and wellbeing inequality, and as such, my thesis represents a unique contribution to the literature. Perhaps more importantly, I have highlighted the likely value of focusing on local variations in wellbeing inequality for the construction of policy instruments. If the proposed amendment to the Local Government Act is passed, and policies addressing local inequalities in wellbeing are created, then New Zealanders will be happier as a result. 
$\mathbf{1 2 3} \mid \mathrm{P}$ a g e

\section{Reference List}

Abdallah, S. \& Quick, A. (2016). Roundtable briefing: Inequalities in wellbeing. New Economics Foundation. Retrieved from www.neweconomics.org

Alesina, A., Di Tella, R., \& MacCulloch, R. (2004). Inequality and happiness: Are Europeans and Americans different? Journal of Public Economics, 88(9/10), 2009-2042

Aslam, A. \& Corrado, L. (2012). The geography of well-being. Journal of Economic Geography, 12(3), 627-649.

Ballas, D. \& Tranmer, M. (2012). Happy people or happy places? A multilevel modelling approach to the analysis of happiness and well-being. International Regional Science Review, 35(1), 70-102.

Becchetti, L., Corrado, L. \& Conzo, P. (2017). Sociability, altruism and well-being. Cambridge Journal of Economics, 41(2), 441-486.

Becchetti, L., Massari, R. \& Naticchioni, P. (2013). The drivers of happiness inequality: Suggestions for promoting social cohesion. Oxford Economic Papers, 66(2), 419-442.

Bentham, J. (1823). An introduction to the principles of morals and legislation. London: W. Pickering.

Berg, M. \& Veenhoven, R. (2010). Income inequality and happiness in 119 nations. In Greve, B. (Ed.), Social Policy and Happiness in Europe, (174-194). Cheltenham: Edward Elgar.

Binder, M. (2015). Volunteering and life satisfaction: A closer look at the hypothesis that volunteering more strongly benefits the unhappy. Applied Economics Letters, 22(11), 874-885.

Bolle, F., Okhrin, Y. \& Vogel, C. (2009). A note on interdependent happiness. The Journal of Socio-Economics, 38, 713-721.

Brulé, G. \& Veenhoven, R. (2017). The '10 excess' phenomenon in responses to survey questions on happiness. Social Indicators Research, 131(2), 853-870.

Buss, D. M. (2000). The evolution of happiness. American Psychologist, 55(1), 15-23.

Cantril, H. (1963). A study of aspirations. Ekistics, 16(93), 121-124.

Charlton, B. G. (1997). The inequity of inequality: Egalitarian instincts and evolutionary psychology. Journal of Health Psychology, 2(3), 413-425.

Chin-Hon-Foei, S. (1989) Life satisfaction in the EC countries, 1975-1984, in R. Veenhoven (ed.) Did the Crisis Really Hurt? Universitaire Pers Rotterdam: Netherlands. 
Clark, A. E., Fléche, S., \& Senik, C. (2016). Economic growth evens out happiness: Evidence from six surveys. Review of Income and Wealth, 62(3), 405-419.

Clark, A. E., Frijters, P., \& Shields, M. A. (2008). Relative income, happiness, and utility: An explanation for the Easterlin Paradox and other puzzles. Journal of Economic Literature, 46(1), 95-144.

Cram, F. (2014). Measuring Māori wellbeing. MAI Journal, 3(1), 18-32.

Dalziel, P. \& Saunders, C. (2014). Wellbeing Economics: Future Directions for New Zealand. Wellington: Bridget Williams Books.

Davidson, R. J. \& Schuyler, B. S. (2015). Neuroscience of happiness. In J. Helliwell, R. Layard, \& J. Sachs (Eds.), World Happiness Report 2015, (88-105), retrieved from http://worldhappiness.report/download/

Dawes, C. T., Fowler, J. H., Johnson, T., McElreath, R. \& Smirnov, O. (2007). Egalitarian motives in humans. Nature, 446, 794-796.

Dawes, C. T., Loewen, P. J., Schreiber, D., Simmons, A. N., Flagan, T., McElreath, R., Bokemper, S. E., Fowler, J. H. \& Paulus, M. P. (2012). Neural basis of egalitarian behaviour. PNAS, 109(17), 6479-6483.

Delhey, J. (2004). Life Satisfaction in an Enlarged Europe. European Foundation for the Improvement of Living and Working Conditions. Luxembourg: Office for Official Publications of the European Communities.

Delhey, J. \& Dragolov, G. (2014). Why inequality makes Europeans less happy: the role of distrust, status anxiety, and perceived conflict. European Sociological Review, 30(2), 151-165.

Delhey, J. \& Kohler, U. (2011). Is happiness inequality immune to income inequality? New evidence through instrument-effect-corrected standard deviations. Social Science Research, 40(3), 742-756.

Dolan, P., Peasgood, T. \& White, M. (2008). Do we really know what makes us happy? A review of the economic literature on the factors associated with subjective well-being. Journal of Economic Psychology, 29(1), 94-122.

Dutta, I. \& Foster, J. (2013). Inequality of happiness in the U.S.: 1972-2010. Review of Income and Wealth, 3, 393-415.

Easterlin, R. A. (1974). Does economic growth improve the human lot? Some empirical evidence. In P. A. David \& M. W. Reder (Eds.), Nations and Households in Economic Growth: Essays in Honor of Moses Abramovitz (89125). New York: Academic Press.

Fahey, T. \& Smyth, E. (2004). Do subjective indicators measure welfare? Evidence from 33 European societies. European Societies, 6(1), 5-27. 
$\mathbf{1 2 5} \mid \mathrm{P}$ a g e

Fehr, E., Bernhard, H. \& Rockenbach, B. (2008). Egalitarianism in young children. Nature, 454(28), 1079-1084.

Ferrer-i-Carbonell, A. \& Frijters, P. (2004). How important is methodology for the estimates of the determinants of happiness? The Economic Journal, 114(497), 641-659.

Fortin, N., Helliwell, J. F., \& Wang, S. (2015). How does subjective well-being vary around the world by gender and age? In J. Helliwell, R. Layard, \& J. Sachs (Eds.), World Happiness Report 2015, (42-75), retrieved from http://worldhappiness.report/download/

Fowler, J. H. \& Christakis, M. A. (2008). Dynamic spread of happiness in a large social network: Longitudinal analysis over 20 years in the Framingham Heart Study. BMJ, 337(a2338), 1-9.

Goff, L., Helliwell, J. H. \& Mayraz, G. (2016). The Welfare Costs of Well-being Inequality. NBER Working Paper No. 21900. Revised December 2016 JEL No. D6,D63,I3

Graham, C. \& Felton, A. (2006). Inequality and happiness: Insights from Latin America. Journal of Economic Inequality, 4(1), 107-122.

Hagerty, M. R. (2000). Social comparisons of income in one's own community: Evidence from national surveys of income and happiness. Journal of Personality and Social Psychology, 78(4), 764-771.

Haller, M. \& Hadler, M. (2006). How social relations and structures can produce happiness and unhappiness: An international comparative analysis. Social Indicators Research, 75(2), 169-216.

Helliwell, J. H. (2003). How's life? Combining individual and national variables to explain subjective well-being. Economic Modelling, 20(2), 331-360.

Helliwell, J. H., Huang, H. \& Wang, S. (2016). The distribution of world happiness. In J. Helliwell, R. Layard, \& J. Sachs (Eds.), World Happiness Report 2016 Update, (8-49), retrieved from http://worldhappiness.report/download/

Helliwell, J. H., Layard, R. \& Sachs, J. D. (2013). Introduction. In J. Helliwell, R. Layard, \& J. Sachs (Eds.), World Happiness Report 2013, (2-7), retrieved from http://worldhappiness.report/download/

Helliwell, J. H. \& Wang, S. (2012). The state of world happiness. In J. Helliwell, R. Layard, \& J. Sachs (Eds.), World Happiness Report, (10-57), retrieved from http://worldhappiness.report/download/

Hutcheson, F. (1726). An Inquiry Into the Original of Our Ideas of Beauty and Virtue. New York: Garland. 
Ifcher, J. \& Zarghamee, H. (2016). Inequality of happiness: Evidence of the compression of the subjective well-being distribution with economic growth. In K. Basu \& J. Stiglitz (Eds.), Inequality and Growth: Patterns and Policy Volume I: Concepts and Analysis, (225-245). New York: Palgrave Macmillan.

Jann, B. (2014). Plotting regression coefficients and other estimates in Stata. The Stata Journal, 14(4), 708-737.

Johnson, T., Dawes, C. T., Fowler, J. H., McElreath, R. \& Smirnov, O. (2009). The role of egalitarian motives in altruistic punishment. Economics Letters, 102, 192-194.

Kalmijn, W. M. (2012). Happiness is not normally distributed: A comment to Delhey and Kohler. Social Science Research, 41(1), 199-202.

Kalmijn, W. M. \& Arends, L. R. (2010). Measures of inequality: Application to happiness in nations. Social Indicators Research, 99(1), 147-162.

Kalmijn, W. M. \& Veenhoven, R. (2005). Measuring inequality of happiness in nations: In search for proper statistics. Journal of Happiness Studies, 6(4), 357-396.

Kalmijn, W. M. \& Veenhoven, R. (2014). Index of Inequality-Adjusted Happiness (IAH) Improved: A Research Note. Journal of Happiness Studies, 15(6), 1259-1265.

Katic, I. \& Ingram, P. (2017). Income inequality and subjective well-being: Toward an understanding of the relationship and its mechanisms. Business and Society, DOI: https://doi.org/10.1177/0007650317701226

Kelley, J. \& Evans, M. D. R. (2017). Societal inequality and individual subjective well-being: Results from 68 societies and over 200,000 individuals, 19812008. Social Science Research, 62, 1-23.

Kramer, A. D. I., Guillory, J. T. \& Hancock, J. E. (2014). Experimental evidence of massive-scale emotional contagion through social networks. Proceedings of the National Academy of Sciences of the United States of America, 111(24), 8788-8790.

Kukutai, T., Sporle, A. \& Roskruge, M. (2017). Subjective whānau wellbeing in Te Kupenga. Social Policy Evaluation and Research Unit. Retrieved from http://www.superu.govt.nz/publication/subjective-whanau-wellbeing-tekupenga

Layard, R. (2016). Promoting Happiness Ethics: The Greatest Happiness Principle. In J. Helliwell, R. Layard, \& J. Sachs (Eds.), World Happiness Report 2016 Update, (50-55-59), retrieved from http://worldhappiness.report/download/

Layard, R., Chisholm, D., Patel, V. \& Saxena, S. (2013). Mental illness and unhappiness. In J. Helliwell, R. Layard, \& J. Sachs (Eds.), World Happiness Report 2013, (38-53), retrieved from http://worldhappiness.report/download/ 
Layard, R., Clark, A. E. \& Senik, C. (2012). The causes of happiness and misery. In J. Helliwell, R. Layard, \& J. Sachs (Eds.), World Happiness Report, (58-89), retrieved from http://worldhappiness.report/download/

Mill, J. S. (1863). Utilitarianism. London: Parker, Son and Bourn.

Morawetz, D., Atia, E., Bin-Nun, G., Felous, L., Gariplerden, Y., Harris, E., Soustiel, S., Tombros, G. \& Zarfaty, Y. (1977). Income distribution and self-rated happiness: Some empirical evidence. The Economic Journal, 87(347), 511-522

Morrison, P. S. (2007). Subjective wellbeing and the city. Social Policy Journal of New Zealand, 31, 74-103.

Morrison, P. S. (2015a). Who cares about income inequality? Policy Quarterly, 11(1), 56-62.

Morrison, P. S. (2015b). Age and inequality. AUT Briefing Papers, retrieved from http://briefingpapers.co.nz/age-and-inequality/

Morrison, P. S. \& Weckroth, M. (2017). Human values, subjective well-being and the metropolitan region. Regional Studies, 52(3), 325-337.

Ngamaba, K. H., Panagioti, M. \& Armitage, C. J. (2018). Income inequality and subjective well-being: a systematic review and meta-analysis. Quality of Life Research, 27(3), 577-596.

Oishi, S., Kesebir, S. \& Diener, E. (2011). Income inequality and happiness. Psychological Science, 22(9), 1095-1100.

Okulicz-Kozaryn, A. (2011). Geography of European life satisfaction. Social Indicators Research, 101(3), 435-445.

Ott, J. C. (2005). Level and inequality of happiness in nations: Does greater happiness of a greater number imply greater inequality in happiness? Journal of Happiness Studies, 6(4), 397-420.

Ott, J. C. (2011). Government and happiness in 130 nations: Good governance fosters higher level and more equality of happiness. Social Indicators Research, 102(1), 3-22.

Ovaska, T. \& Takashima, R. (2010). Does a rising ride lift all the boats? Explaining the national inequality of happiness. Journal of Economic Issues, 44(1), 205223.

Paine, T. (1792). Rights of Man. London: Printed for J.S. Jordan

Parliamentary Counsel Office. (2018). Local Government (Community Well-being) Amendment Bill, retrieved from http://www.legislation.govt.nz/bill/government/2018/0048/latest/LMS30972.ht $\underline{\mathrm{ml}}$ 
Pittau, M. G., Zelli, R. \& Gelman, A. (2010). Economic disparities and life satisfaction in European regions. Social Indicators Research, 96(2), 339-361.

Quick, A. (2015). Inequalities in wellbeing: Challenges and opportunities for research and policy. New Economics Foundation. Retrieved from www.neweconomics.org

Rand, D. G., Brescoll, V. L., Everett, J. A. C., Capraro, V. \& Barcelo, H. (2016). Social heuristics and social roles: Intuition favors altruism for women but not for men. Journal of Experimental Psychology: General, 145(4), 389-396.

Reeskens, T., \& Hooghe, M. (2008). Cross-cultural measurement equivalence of generalized trust. Evidence from the European Social Survey (2002 and 2004). Social Indicators Research, 85(3), 515-532.

Ripahn, R. T., Serfling, O. (2005). Item non-response on income and wealth questions. Empirical Economics, 30(2), 521-538.

Robinson, D. \& Williams, T. (2001). Social capital and voluntary activity: Giving and sharing in Māori and non-Māori society. Social Policy Journal of New Zealand, 17, 52-71.

Robinson, W. S. (1950). Ecological correlations and the behaviour of individuals. American Sociological Review, 15(3), 351-357.

Rosenberg, M. (1957). Misanthropy and attitudes towards international affairs. The Journal of Conflict Resolution, 1(4), 340-345.

Sachs, J. D. (2015). Investing in social capital. In J. Helliwell, R. Layard, \& J. Sachs (Eds.), World Happiness Report 2015, (152-167), retrieved from http://worldhappiness.report/download/

Schwarze, J. \& Härpfer, M. (2007). Are people inequality averse, and do they prefer redistribution by the state? Evidence from German longitudinal data on life satisfaction. The Journal of Socio-Economics, 36(2), 233-249.

Senik, C. (2004). When information dominates comparison, learning from Russian subjective panel data. Journal of Public Economics, 88(9-10), 2099-2123.

Sibley, C. G., Harré, N., Hoverd, W. \& Houkamau, C. A. (2011). The gap in the subjective wellbeing of Māori and New Zealand Europeans widened between 2005 and 2009. Social Indicators Research, 104(1), 103-115.

Simmons, W. O. \& Emanuele, R. (2007). Male-female giving differentials: Are women more altruistic? Journal of Economic Studies, 34(6), 534-550.

Stevenson, B. \& Wolfers, J. (2008) Happiness inequality in the United States. Journal of Legal Studies, 37(2), 33-79. 
129|P a g e

Stiglitz, J. E., Sen, A. \& Fitoussi, J. P. (2009). Report by the commission on the measurement of economic performance and social progress. (The Commission on the Measurement of Economic Performance and Social Progress (CMEPSP)). Retrieved from http://ec.europa.eu/eurostat/documents/118025/118123/Fitoussi+Commission+ $\underline{\text { report }}$

Tesfazghi, E. S., Martinez, J. A. \& Verplanke, J. J. (2010). Variability of quality of life at small scales: Addis Ababa, Kirkos sub-city. Social Indicators Research, 98(1), 73-88.

Tobler, W. R. (1970). A Computer Movie Simulating Urban Growth in the Detroit Region. Economic Geography, 46, 234-240.

Tomes, N. (1986). Income distribution, happiness and satisfaction: A direct test of the interdependent preferences model. Journal of Economic Psychology, 7(4), 425-446.

Ura, K., Alkire, S. \& Zangmo, T. (2012). Case Study: Bhutan, gross national happiness and the GNH index. In J. Helliwell, R. Layard, \& J. Sachs (Eds.), World Happiness Report, (58-89), retrieved from http://worldhappiness.report/download/

Veenhoven, R. (2000). Well-being in the welfare state: Level not higher, distribution not more equitable. Journal of Comparative Policy Analysis: Research and Practice, 2, 91-125.

Veenhoven, R. (2005). Return of inequality in modern society? Test by dispersion of life-satisfaction across time and nations. Journal of Happiness Studies, 6(4), 457-487.

Veenhoven, R. (2012). The medicine is worse than the disease: Comment on Delhey and Kohler's proposal to measure inequality in happiness using 'instrumenteffect-corrected' standard deviations. Social Science Research, 41(1), 203-205.

Veenhoven, R. \& Kalmijn, W. M. (2005). Inequality-adjusted happiness in nations: Egalitarianism and utilitarianism married in a new index of societal performance. Journal of Happiness Studies, 6(4), 421-455.

Wilkinson, R. \& Pickett, K. (2009). The Spirit Level: Why More Equal Societies Almost Always Do Better. London: Penguin Books.

Winkelmann, R. (2009). Unemployment, social capital, and subjective well-being. Journal of Happiness Studies, 10(4), 421-430. 


\section{Appendix A: Technical Information on the Three Surveys}

This appendix contains information on the data collection process for each of the three surveys, and details how the data was manipulated to create the variables used in my analysis. While not essential to interpreting the information within the thesis, this appendix is useful for reference should a greater depth of knowledge about the surveys or variables be desired.

\section{A.1: The Quality of Life Survey (QoLS)}

\section{Data Collection Methodology}

In 2012, the QoLS team for the first time used a sequential mixed-method methodology, which was subsequently used in 2014 as well. This approach to data collection relied on self-completion of the respondents, with respondents being encouraged to complete the survey online before being sent a paper copy. Given that this method does not require telephone access, the survey team drew their sample from the entire electoral roll, rather than just those who had been successfully linked with a telephone number. As such, the 2012 and 2014 QoLS were able to reach a wider number of people than telephone based surveys.

As for changes in the questions presented in the surveys, from 2012 to 2014 the survey questions remained largely the same. Any differences between the two survey years will be discussed later in this Appendix in the 'Variables' section.

\section{The Target Population}

The target population of the QoLS was residents aged 18 years and living in the six cities participating in both years. Quotas were set for age, ethnicity, sex, city, and ward.

In 2012, the target sample population was set at 5,700 responses, with the actual responses falling short at 5,151. Auckland, Hutt City and Porirua all contributed to this, failing to reach their individual targets. Data collection was conducted between August and October of 2012. The response rate for the 2012 survey was 33\%.

In 2014, the target sample population was 4,500. This target was met, with a total of 5,295 responses collected. Christchurch was the only city to fall short of its 
target in 2014, obtaining 488 out of the desired 500 responses. The survey was conducted between June and July of 2014, and the response rate was only $30 \%$.

\section{$\underline{\text { Variables for Analysis }}$}

The QoLS data was sometimes inconsistent in terms of coding from year to year, and variable labels were often different. During the data cleaning process, a few new variables were generated, and many of the existing variables of interest were edited in order to make them suitable for analysis. The following section will provide detail on the editing and generation process of the QoLS variables most relevant for analysis.

\section{Original Dataset Variables}

A few variables were consistent between 2012 and 2014 in the way that they were formatted within the questionnaire. However, the way in which the data was inputted differed, meaning that most of the variables needed to be transferred to Excel for data cleaning purposes. The cleaning process primarily involved recoding or collapsing the variables to ensure that the values and value labels were consistent throughout the years. This section will discuss the variables that were only recoded, rather than those newly generated or completely transformed for analysis.

The life satisfaction (the dependent variable of interest in this thesis) was consistent between 2012 and 2014.The question itself reads: "Taking everything into account, how satisfied or dissatisfied are you with your life in general these days?". Respondents had the option of answering on a 5-point Likert scale, ranging from "Very dissatisfied" ('1') to "Very satisfied" ('5'). The SWB inequality variables will be discussed later in this appendix.

Following the SWB variable, the most important variables to consider in this thesis are the geographical ones, which are used in the creation of our SWB inequality indicators. In contrast with both the NZGSS and Te Kupenga, use of the QoLS did not require the generation of a completely new geographic variable.

Both 2012 and 2014 contained a 'city' variable, indicating the respondent's city of residence. Options included six cities: Auckland, Wellington, Porirua, Hutt City, Christchurch and Dunedin.

In addition to the city variable, both years of the QoLS include a variable assigning each respondent with an electoral ward. This variable is named 'ward'. While 
the variable itself was not new, each ward was coded differently in each survey, requiring a great deal of data cleaning to ensure that the variable was consistent across the dataset. In the dataset, Auckland contains 20 wards, Wellington contains five wards, Hutt City contains six wards, Porirua contains three wards, Christchurch contains seven wards, and Dunedin contains five wards. Technically, the Auckland 'wards' identify community or local board areas: but for the sake of clear communication they are referred to as 'wards' as well throughout this thesis. A total of 46 wards are present for analysis.

Given that the ward variable was to be used in the generation of the SWB inequality variables, it was important to ensure that each ward contained enough individual observations to allow some significant amount of variation. As such, any ward with less than 30 respondents in them in either of 2012 or 2014 were dropped from the analysis. The only ward to fit this criteria was the Banks Peninsula ward of Christchurch, which had a total of only 12 responses in 2012, and 14 responses in 2014. This left a total of 45 wards for analysis.

\section{$\underline{\text { Generated Variables }}$}

The key variable that has been generated from the QoLS for this research is a measure of life satisfaction inequality. The same procedure for generating the inequality variable is used in each survey.

The ward variable was used as the grouping indicator with which to generate the standard deviation values for life satisfaction. The dataset was collapsed (using the 'collapse' command) to create a new dataset containing just the life satisfaction variables prepared for the ecological regressions. This new dataset has only 90 observations remaining, one per ward for each year. Every individual ward has a mean SWB value 'meanls' and a SWB life satisfaction value 'sdls' assigned as part of this process. Table A.1 displays the mean and standard deviation (in brackets) of life satisfaction, rounded to $3 \mathrm{dp}$.

For the analysis of The Primary Hypothesis the same process was used to generate life satisfaction inequality using the city variable instead of the ward variable. At the city level in the collapsed dataset the mean life satisfaction variable is labelled 'meanlscity' and the standard deviation life satisfaction variable is labelled 'sdlscity'. 
133 | P a g e

In the individual dataset the city level standard deviation life satisfaction variable is labelled 'sdcitylifesat'.

Generating the SWB inequality variable at the individual level followed the same process in each survey as well. The 'egen' command was used on the ward variable in order to create the standard deviation of life satisfaction variables: 'sdlife12' and 'sdlife14', for 2012 and 2014 respectively.

Table A. 1

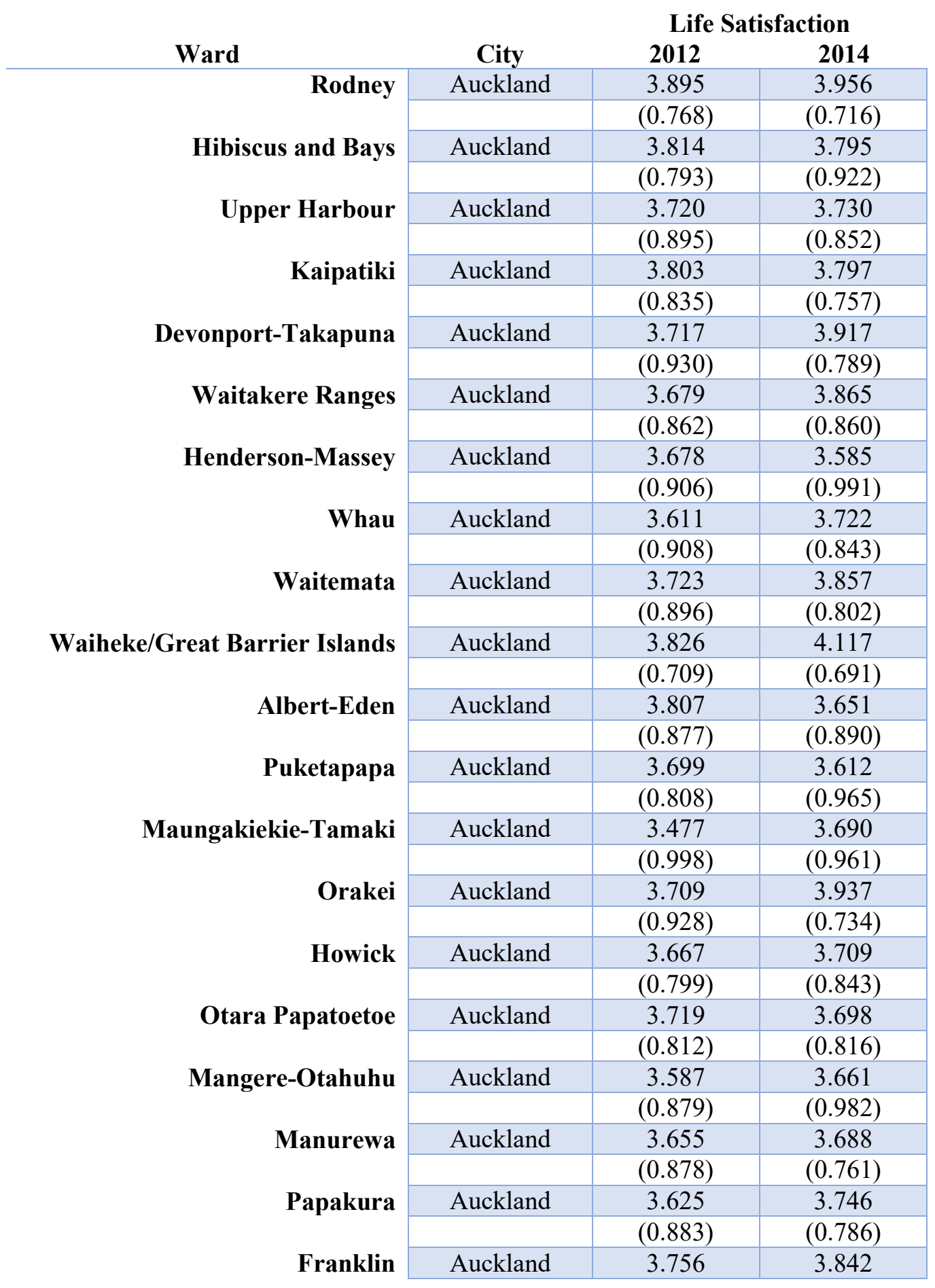




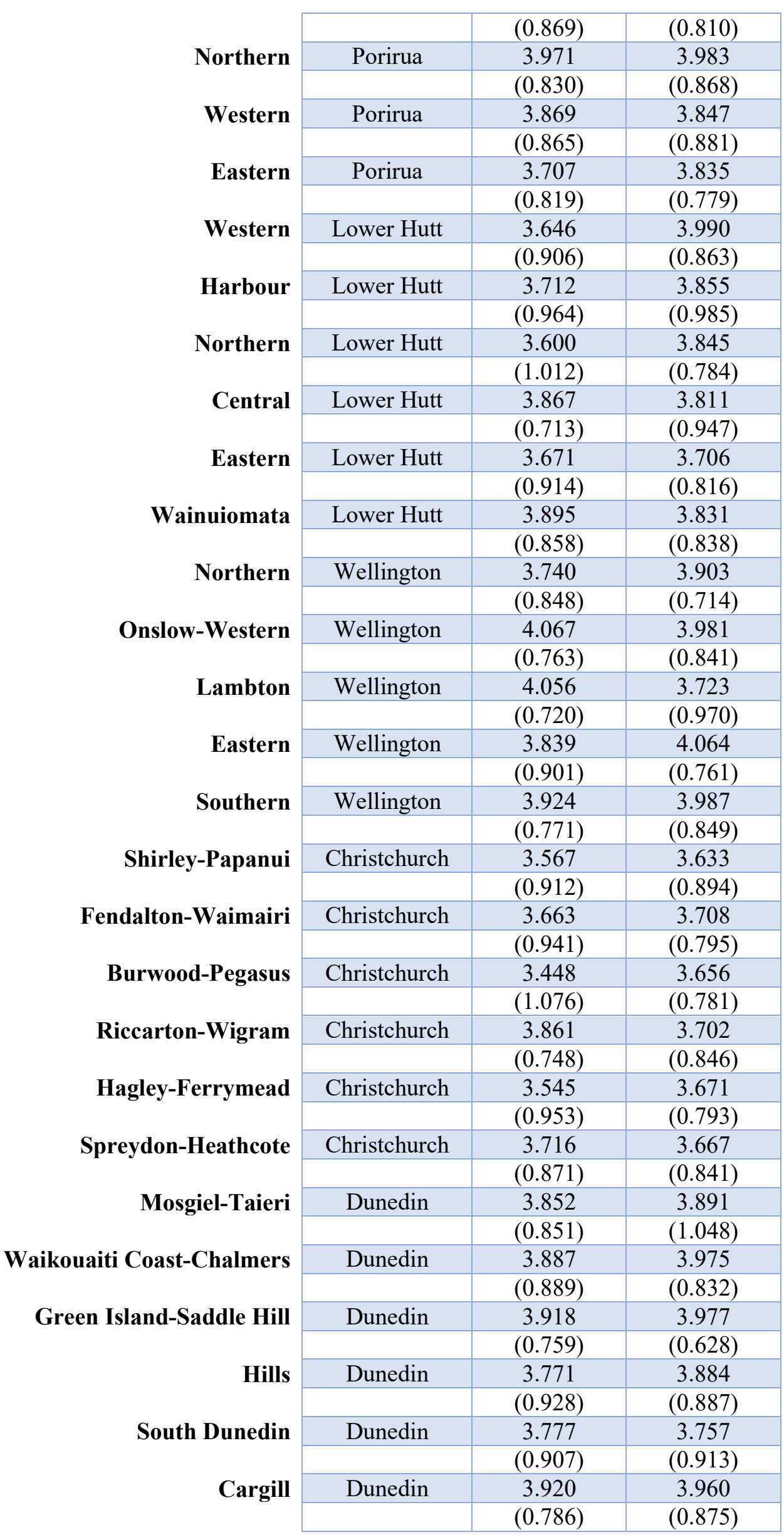


$\mathbf{1 3 5} \mid \mathrm{P}$ a g e

\section{Controlling Variables}

Given the time consuming nature of editing each variable and making them consistent between the two years, only a select number of controlling variables were incorporated into the QoLS dataset. These variables were chosen on the basis that they have been shown to have the greatest impact upon SWB in the literature. The control variables used in the QoLS include age, ethnicity, social isolation, sense of community, subjective health, employment, education, and income. The following paragraphs will provide detail on the form of these variables, as well as briefly covering any alterations made to them.

The age variable in the QoLS originally consisted of 12 age groups, and is named 'agegrp'. The youngest age group was for those below the age of 18 , and the oldest age group for those over 65 years of age. As only eight individuals were younger than 18, they were added to the second youngest age group (18-19 years of age), bringing the total down to 11 groups in the variable. Apart from the youngest age group, 'less than 20 years old' and the oldest age group ' $65+$ ', all of the age groups consisted of five year bands. The mean age group in 2012 was the 45-49 year old group and in 2014 was the 40-44 year old group. From this age group variable, a new one called 'agedecade' was created, with age groups in decades instead of five year age groups. This new variable has six age groups in total, ranging from 'less than 20 years old' at the bottom, to ' $60+$ ' at the top.

Ethnicity in the QoLS is measured using a simple binary variable indicating whether the respondent identifies as New Zealand European or not. The variable is labelled 'nzeuro' with ' 1 ' indicating that the respondent is New Zealand European, and ' 0 ' that they are not. In 2012 there are 3,745 people who identify as New Zealand European (72.87\%) and 1,394 who do not (27.13\%). The equivalent numbers 2014 are $3,682(69.83 \%)$ and $1,591(30.17 \%)$.

The sex variable was a simple binary indicator in each year, asking individuals to choose "Male" or "Female". In the current dataset the sex variable is labelled 'sex', with male represented as ' 1 ' and female as ' 0 '. Unfortunately, neither year gave respondents the option of choosing a non-binary gender. The 2012 data contains 2,773 females (53.96\%) and 2,366 males (46.04\%), while the 2014 data contains 2,847 females (54.04\%) and 2,421 males (45.96\%), indicating a reasonably good gender balance in respondents. 
Social capital, or regular close social interaction with others, is one of the most effective ways that an individual can increase their SWB. As such, controlling for an individual's social connectivity can be useful. In particular social connectivity is relevant for this research, as more social individuals are likely to have a better idea of the wellbeing distribution as a result of their increased frequency of interactions. Social capital and connectivity is measured in a variety of ways across different surveys. Luckily, the QoLS contains two useful measures which are fairly representative of social connections in the local community. The first is a rating of personal social isolation or loneliness, and the second relates to an individual's sense of community.

The social isolation question was asked in the QoLS in both years and was phrased: "In the last 12 months how often, if ever have you felt lonely or isolated". Respondents had the option of replying with "Always" ('1'), "Most of the time" ('2'), "Sometimes" ('3'), "Rarely" ("4') or "Never" ('5'). The variable in the current research is labelled 'isolation', and has been reverse coded so that a higher score on the variable is associated with greater isolation ("Always" is now ' 5 ' and "Never" is ' 1 '). The mean score for isolation is 2.07 in 2012, and 2.04 in 2014, indicating that most individuals in the dataset only feel lonely or isolated rarely.

The other measure of social connectedness used in this survey relates to an individual's sense of community in their local area. This variable has the potential to be a particularly important control, as it is likely that those with a greater sense of community will also care more and be more aware of the SWB distribution within it. In both years survey participants were asked to rank their agreement or disagreement on a 5-point Likert scale in response to the following statement "I feel a sense of community with others in my neighbourhood". The variable is labelled 'communitysense' in the current research. On the Likert scale, "Strongly disagree" is ' 1 ' and "Strongly agree" is ' 5 ', meaning that higher scores are associated with a stronger sense of community. The mean score on the communitysense variable for 2012 is 3.45 , and for 2014 is 3.41 , indicating that most of the respondents lean towards some degree of agreement that they feel a sense of community.

The subjective health variable in the QoLS is labelled 'health'. The question itself remained the same in both years of the data and asked "In general how would you rate your health", giving individuals the option to respond with either "Poor" ('1'), "Fair" ('2'), "Good" ('3’), "Very good" ('4') or "Excellent" ('5'). As such, a higher 
score on the 'health' variable corresponds to better self-evaluated health. In 2012 the mean subjective health score is 3.36 , while in 2014 the mean is 3.42 .

The employment status variable in the QoLS was the same in both years of the data. Respondents were asked "Which of the following best describes your current employment status?". Employment was also defined for the respondent as "you undertake work for pay, profit or other income, or do any work in a family business without pay". Respondents had the option of choosing between "Employed full time (for 30 hours or more per week)" ('1'), "Employed part time (for less than 30 hours per week)" ('2'), "Not in paid employment and looking for work" ('3'), or "Not in paid employment and not looking for work (e.g. full-time parents, retired person)” (‘4'). Respondents were also explicitly given the option to refuse the question, although this was not coded in the dataset. In 2012, $6.4 \%$ of people did not answer the question. Of these, $52.06 \%$ are employed full time, $16.34 \%$ are employed part time, $5.97 \%$ are unemployed and looking for work, and $25.63 \%$ are unemployed and not looking for work. In 2014, $6 \%$ of people did not answer the employment question. Of these, $55.83 \%$ are employed full time, $18.33 \%$ are employed part time, $6.59 \%$ are unemployed and looking for work, and $19.27 \%$ are unemployed and not looking for work. These percentages indicate that there is considerable variation in employment status between the two years of the data.

Education in the QoLS was initially a categorical variable with 15 separate categories. However, the literature generally finds an unclear relationship between life satisfaction and education when so many separate categories are used, so a common approach is to look at the impacts of university education upon life satisfaction. As such, a binary variable representing tertiary education was created by combining the four tertiary education categories and coding them as ' 1 ', while coding the rest as ' 0 '. The variable is named 'colled'. The tertiary education categories were "Teaching or Nursing Certificate/Diploma", "Bachelor's degree", "Postgraduate degree" and "Postgraduate diploma". In 2012, a total of 1,913 people have a university education (38.31\%), while in 2014 there are 2,173 people with a university education (41.77\%).

The personal income variable in the QoLS is labelled 'incomepers', and indicates the income group to which the respondent belongs. The first group is "Loss/No Income" (coded as '0'), followed by "Less than $\$ 10,000$ " ('1'). After the first two groups, each group increases by $\$ 10,000$ from " $\$ 10,001-\$ 20,000 ”$ ('3’) to 
“\$60,001 - \$70,000” ('7'). The final two groups are " $\$ 70,001-\$ 100,000 ”$ ('8') and "More than \$100,000" ('9'). The mean score for the income variable is 4.57 in 2012, and 4.78 in 2014 , both somewhere between the $\$ 30,001-\$ 40,000$ and $\$ 40,001$ $\$ 50,000$ groups.

\section{A.2: The New Zealand General Social Survey}

The NZGSS is conducted biannually by Statistics New Zealand to provide statistics about social wellbeing, with the aim of informing decision making at the government and wider community levels. Each NZGSS wave consists of two surveys: the personal questionnaire and the household questionnaire. This thesis draws on the personal questionnaire. One eligible individual per household is randomly selected to participate in the individual questionnaire/

\section{The Target Population}

The NZGSS target population is the usually resident population of New Zealand who aged 15 years and over and who live in private dwellings. Two groups are excluded from this target population which are relevant to consider when forming geographic variables. Firstly, people living on offshore islands are not included in the sample, with the exception of Waiheke Island. Secondly, New Zealand usual residents who live in remote areas that are difficult or costly to access were also not surveyed. For the most part however, the survey target population is reasonably representative of wider New Zealand.

\section{$\underline{\text { Data Collection Methodology and Reliability of Data }}$}

The NZGSS data collection methodology was reasonably consistent across 2008, 2010, and 2012. Data was collected in all three years using computer-assisted personal interviews (CAPI), lasting an average of 45 minutes in each year. CAPI involves researchers interviewing respondents, using showcards for the questions, and inputting the data on to computers. With the exception of a few added questions not relevant to this thesis, the survey structure remained the same throughout 2008, 2010 and 2012 .

Response rates in all three years were high. In 2008, the survey response rate was $83 \%$, with a total of 8,721 responses. In 2010 the response rate was $81 \%$, with a total of 8,550 responses. In 2012 , the response rate was at its lowest at $78 \%$, with only 
139| P a g e

8,462 responses. The three years of the NZGSS have the largest samples size of any of the survey years analysed in this thesis.

It is important to also consider the period of data collection for each survey, in order to discern whether outside events may have had an influence on the sample or the data. The 2008 survey was conducted from April 2008 to March 2009; the 2010 survey was conducted from April 2010 to March 2011; and the 2012 survey was conducted from April 2012 to March 2013. The most significant event in this period was the Christchurch earthquake on the $22^{\text {nd }}$ of February, 2011. As a result of this event, workers could not collect data in certain parts of the Canterbury region in the final month, meaning that the 2010 data has fewer responses from Canterbury than initially expected. The earthquake did not impact data collection for the 2012 dataset.

\section{$\underline{\text { Variables for Analysis }}$}

Given that the data was collected and processed by researchers from a different organisation, a greater amount of time than usual had to be dedicated to data exploration, cleaning and manipulation in order to prepare the data for analysis within this thesis. Further complicating this process is the need to make data from three different years consistent, with variables that often changed in labelling and formatting from year to year. As a result, the variables used in this research are a combination of those originally available in the survey and additional variables generated. Initially, the three years were combined to form a pooled dataset. However, statistically significant differences between the years for a number of variables prompted the decision to analyse each year separately in the final analysis.

\section{$\underline{\text { Original Dataset Variables }}$}

A number of variables were consistent across the 2008, 2010 and 2012 versions of the dataset, and required only minor alterations when comparing the different years. The append function of Stata takes any variables with the same name over various separate datasets (such as different years) and combines them in a new dataset, making them easier to compare during analysis. Any variables with different names are still included in the new dataset, but only have observations for the year that they were recorded. The following few paragraphs will focus on discussing the NZGSS variables most relevant to the hypotheses of the current research which required little or no modification to maintain consistency between the years. 
The dependent variable of interest in the NZGSS is the survey's life satisfaction question, and as such it deserves greater depth in explanation. Across the three years, the life satisfaction question remained the same. The question is prefaced by "I' $m$ now going to ask you a very general question about your life. This includes all areas of your life, not just what we have talked about so far." This was followed by the presentation of a showcard with the different responses on it, and the question "Looking at showcard 1, how do you feel about your life as a whole right now?" Responses were recorded on a 5-point Likert scale, and ranged from "very satisfied" (coded as '11') to "very dissatisfied" ('15'). These values were reverse coded in this research, so that a higher number is indicative of greater life satisfaction, consistent with the majority of existing literature. Those who were coded as "don't know" ('88') or refused ('99') were recoded as missing observations. As such, the final range of the life satisfaction variable used in this research ranged from "very dissatisfied" ('1') to "very satisfied" ('5'). In 2008 the mean life satisfaction score is 4.07, in 2010 it is 4.09, and in 2012 it is 4.07 once again.

Other variables relevant to the four hypotheses include some representation of an individual's level of altruism, wellbeing inequality, perceptions of fairness and demographic characteristics. Wellbeing inequality is measured by geographic area, and will discussed in the following sub-section on newly generated variables. The demographic characteristics will be discussed in the following section on controlling variables. However, there are a few questions which roughly correspond to an individual's level of altruism and perceptions of fairness in society, and which will therefore be used in the analysis.

The first variable relevant to altruism is a measure of whether an individual volunteers. Volunteering entails sacrificing one's own time to work for the benefit of others, making it representative of altruistic virtues. The question is labelled 'volunteer', and asked the respondent if "In the last four weeks, did you do any voluntary work for a group or organisation?" Respondents simply replied with "yes" (coded as '1') or "no" (coded as '0'). Across the combined NZGSS dataset, 6 respondents were coded as 'don't know', and were subsequently recoded as missing. In the 2008 data a total of $2,794(32.18 \%)$ of individuals had volunteered recently, in 2010 the total is $2,695(31.67 \%)$, and in 2012 the total is at its lowest at 2,512 (29.82\%). 
In order to provide more than one measure to represent an individual's altruistic tendencies, a second variable will also be analysed. This variable has been labelled 'helper', and is indicative of a much broader set of altruistic tasks than the volunteer variable. In the NZGSS the question asked respondents "[Apart from the voluntary work that you told me about earlier], in the last four weeks did you give any help that you did not get paid for, [to people who don't live with you] anyone?", to which they again responded with either "yes" (' 1 ') or "no" ('0'). The 13 respondents across the three years who replied with 'don't know' were recoded as missing. Note that the helping question is explicitly distinguished from the volunteering question (which preceded it in the surveys) in order to ensure that the same activities are not being recorded twice. As expected, a number of individuals were both volunteers and helpers: the correlation between the two variables is .17 in 2008, .14 in 2010, and .15 in 2012. In 2008, a total of 5,522 people reported that they provided help recently (63.62\%), in 2010 the total is 5,521 (64.91\%), while in 2012 the total is 5,228 (62.05\%). Evidently, while only a small proportion of people have volunteered recently, the majority of individuals have recently helped someone that they do not live with.

The final hypothesis of this thesis concerns the impact that individual perceptions of fairness may have upon sensitivity to SWB. The NZGSS 2008, 2010 and 2012 pose a number of different questions regarding the individual's perceived fairness of different aspects of New Zealand's society. Respondents were asked whether they "think staff at various organisations in New Zealand accept and tolerate different groups" in reference to the following groups: 'staff at your local council', 'the police in your area', 'judges and other staff at law courts', 'staff at government departments', 'your local doctors', 'staff at other health services in your area', 'staff at the schools in your area', 'staff at other education facilities like polytech or university in your area', 'local employers', and 'staff at local shops and other services'. Respondents were asked on a 5-point scale from "strongly agree" ('11') to "strongly disagree" ('15') in response to the statement "(organisation) treat everyone fairly, regardless of what group they are from.", where 'organisation' is replaced with one of the above categories. There is a separate variable for each 'organisation'. The variables have been recoded so that "strongly agree" is ' 5 ' and "strongly disagree" is ' 1 '. Unlike the other variables in the NZGSS, a large proportion of each of these questions were coded as “don’t know” ('89'). As such, created variables kept “don’t know” responses as ' 89 ' in the data. 
Each perceptions of fairness variable was also converted into a binary representing trust in that 'organisation' to be fair. In these instances, responses of ' 4 ' and ' 5 ' were recoded to ' 1 ', representing those who do trust in that organisation to be fair, and '1', '2', '3' and '89' were recoded to '0', representing those who do not trust in that organisation to be fair. There are two sets of 10 variables representing perceptions of fairness, but their labels will not be reproduced here, as they are not individually used in the analysis.

In order to create a singular variable representing an individual's perceptions of fairness in society, the average response of the individual to all 10 of the questions was taken. In order to generate this variable, "don't know" ('89') responses were recoded to be "neither agree nor disagree" ('3') as this was the response that was most often statistically not significantly different from "don't know". The new variable is labelled 'avgtrusttobefair', and has a mean value of 3.64 in 2008, 3.63 in 2010, and 3.62 in 2012. A binary labelled 'avgtrustbinary' was created from 'avgtrusttobefair', with values from ' 1 ' to ' 3.49 ' recoded as ' 0 ', and ' 3.5 ' to ' 5 ' recoded as ' 1 '. A total of 5,800 people $(67.13 \%)$ trusted organisations to be fair on average in 2008, whilst in 2010 and 2012 these totals were 5,695 (67.13\%) and 5,576 (66.31\%) respectively.

\section{Generated Variables}

The geographic variables in the NZGSS were 'region' and 'urban'. The variable 'urban' consists of a simple code of whether an individual lived in one of four types of urban area: "main urban", "secondary urban", "minor urban" or "rural". The majority of responses (nearly three quarters) are from main urban areas, while the remainder of responses are spread more evenly between the other categories. The variable 'region' consists of six categories of regions around New Zealand, varying significantly in size. The regions are "Auckland", "Wellington", "Northland Group", "Rest of North Island", "Canterbury" and "Rest of South Island". The responses were spread fairly evenly throughout the categories, but the largest populations were from 'Auckland' and the 'Rest of North Island' respectively.

While these categories are useful on their own, they do not provide enough groups to examine the relationship between SWB and local SWB inequality in a meaningful way. The 'group' command was used in Stata to combine the urban and region variables, creating a new variable called 'reg1' (referred to as 'urban-region' in the text). Instead of having two geographic variables with four and six different 
$\mathbf{1 4 3} \mid \mathrm{P}$ a g e

categories, this allowed the usage of a new variable which contained 24 distinct geographic areas. These areas and the labels associated with them are summarised in Table 5.1.

This new geographic variable 'reg1' was used as the grouping variable with which to generate standard deviation values. The dataset was collapsed so that only 24 observations remained for each of the three years, one for each of the new combined region and urban areas. A $25^{\text {th }}$ group was generated in each year for the 65 individuals that did not have a value for the 'urban' variable; this group was recoded as missing from the data due to their unknown location related to the others. Each of these regions had a mean life satisfaction value and a standard deviation life satisfaction value assigned. Table A.2 displays the mean life satisfaction values and standard deviation life satisfaction values (in brackets) corresponding to each group within reg1 for each year, rounded to $3 \mathrm{dp}$. In the collapsed dataset, the variable representing mean life satisfaction for 2008 is labelled 'meanls08', for 2010 is 'meanls10', and for 2012 is 'meanls12'. Standard deviation in the collapsed dataset is labelled 'sdls08' for 2008, 'sdls10' for 2010, and 'sdls12' for 2012. 


\begin{tabular}{|c|c|c|c|c|}
\hline Table A. 2 & Main Urban & $\begin{array}{c}\text { Secondary } \\
\text { Urban }\end{array}$ & $\begin{array}{l}\text { Minor } \\
\text { Urban }\end{array}$ & Rural \\
\hline \multicolumn{5}{|l|}{2008} \\
\hline \multirow[t]{2}{*}{ Auckland } & 4.045 & 3.925 & 4.083 & 4.346 \\
\hline & $(0.847)$ & $(0.917)$ & $(0.900)$ & $(0.764)$ \\
\hline \multirow[t]{2}{*}{ Wellington } & 4.060 & 4.108 & 4.533 & 4.500 \\
\hline & $(0.853)$ & $(0.938)$ & $(0.640)$ & $(0.707)$ \\
\hline \multirow[t]{2}{*}{ Northland Group } & 3.991 & 3.979 & 4.097 & 4.067 \\
\hline & $(0.939)$ & $(0.911)$ & $(0.753)$ & $(0.913)$ \\
\hline \multirow[t]{2}{*}{ Rest of North Island } & 4.123 & 3.959 & 4.068 & 4.162 \\
\hline & $(0.828)$ & $(0.912)$ & $(0.946)$ & $(0.876)$ \\
\hline \multirow[t]{2}{*}{ Canterbury } & 4.065 & 3.985 & 4.174 & 4.218 \\
\hline & $(0.853)$ & $(0.764)$ & $(0.760)$ & $(0.759)$ \\
\hline \multirow[t]{2}{*}{ Rest of South Island } & 4.086 & 4.097 & 4.160 & 4.018 \\
\hline & $(0.881)$ & $(0.960)$ & $(0.926)$ & $(0.918)$ \\
\hline \multicolumn{5}{|l|}{2010} \\
\hline \multirow[t]{2}{*}{ Auckland } & 4.085 & 3.952 & 3.952 & 4.163 \\
\hline & $(0.804)$ & $(0.987)$ & $(1.244)$ & $(0.965)$ \\
\hline \multirow[t]{2}{*}{ Wellington } & 4.109 & 3.947 & 4.167 & 4.222 \\
\hline & $(0.846)$ & $(0.854)$ & $(0.835)$ & $(0.892)$ \\
\hline \multirow[t]{2}{*}{ Northland Group } & 4.049 & 4.070 & 3.929 & 4.199 \\
\hline & $(0.898)$ & $(0.863)$ & $(0.986)$ & $(0.911)$ \\
\hline \multirow[t]{2}{*}{ Rest of North Island } & 4.059 & 4.069 & 4.074 & 4.022 \\
\hline & $(0.877)$ & $(0.908)$ & $(0.935)$ & $(0.939)$ \\
\hline \multirow[t]{2}{*}{ Canterbury } & 4.026 & 3.990 & 4.193 & 4.306 \\
\hline & $(0.854)$ & $(0.978)$ & $(0.776)$ & $(0.772)$ \\
\hline \multirow[t]{2}{*}{ Rest of South Island } & 4.084 & 4.160 & 4.192 & 4.292 \\
\hline & $(0.819)$ & $(0.805)$ & $(0.838)$ & $(0.802)$ \\
\hline \multicolumn{5}{|l|}{2012} \\
\hline \multirow[t]{2}{*}{ Auckland } & 4.074 & 4.367 & 3.939 & 4.043 \\
\hline & $(0.820)$ & $(0.718)$ & $(0.966)$ & $(0.928)$ \\
\hline \multirow[t]{2}{*}{ Wellington } & 4.111 & 4.034 & 4.019 & 4.083 \\
\hline & $(0.828)$ & $(0.765)$ & $(0.866)$ & $(0.974)$ \\
\hline \multirow[t]{2}{*}{ Northland Group } & 4.063 & 4.061 & 3.944 & 4.063 \\
\hline & $(0.862)$ & $(0.876)$ & $(0.776)$ & $(0.886)$ \\
\hline \multirow[t]{2}{*}{ Rest of North Island } & 4.078 & 3.963 & 3.990 & 4.205 \\
\hline & $(0.853)$ & $(0.957)$ & $(0.893)$ & $(0.851)$ \\
\hline \multirow[t]{2}{*}{ Canterbury } & 4.019 & 4.170 & 4.041 & 4.170 \\
\hline & $(0.924)$ & $(0.773)$ & $(0.840)$ & $(0.798)$ \\
\hline \multirow[t]{2}{*}{ Rest of South Island } & 4.094 & 3.969 & 4.215 & 4.218 \\
\hline & $(0.857)$ & $(0.825)$ & $(0.801)$ & $(0.806)$ \\
\hline
\end{tabular}


The process for generating the standard deviation of life satisfaction at the individual level was similar to that used at the aggregate level. The dataset was not collapsed into a smaller one, instead remaining with all of its original observations intact. The same geographic groupings in reg1 were again used, this time in conjunction with the 'egen' command in Stata. This created a new variable for each year representing the standard deviation of life satisfaction in each 'reg1' group in that year. Variable names remained the same as those used in the collapsed dataset: 'sdls08', 'sdls10' and 'sdls12' for 2008, 2010 and 2012 respectively.

Within the standard deviation variables, each individual was assigned a value corresponding with the standard deviation in life satisfaction within their area. These standard deviations were the same as those in the collapsed dataset displayed in Table 5.1. For example, in the year 2008 every individual living in 'Main Urban Auckland' would be assigned a standard deviation ('sdls08') value of .847, while every individual in 'Secondary Urban Wellington' would be assigned a value of .938. 65 individuals were not assigned a value for the 'urban' variable, and so their observations were coded as missing for the analysis.

A number of 'reg1' groups were excluded from the final analysis owing to small sample size. In order to allow for sufficient variation in life satisfaction, any group with 30 or fewer responses in any one year was excluded from the analysis in that year. The groups not analysed are: Secondary Urban Auckland (2012), Minor Urban Auckland (2008, 2010), Rural Auckland (2012), Minor Urban Wellington (2008, 2010) and Rural Wellington $(2008,2010,2012)$. After these observations are excluded, 21 distinct groups remain in each of 2008, 2010, and 2012.

For addressing the hypotheses that required a larger geographic region or a higher sample size, the same processes were used to generate life satisfaction inequality using the basic 'region' variable instead of the 'reg1' generated variable. When doing so, variables were created labelled 'sdlsregion08', 'sdlsregion10', 'sdlsregion12' for standard deviation life satisfaction and 'meanlsregion08', 'meanlsregion10' and 'meanlsregion12' for mean life satisfaction. 


\section{Controlling Variables}

A range of other variables were included from the original data in order to control for known factors that may have an influence on life satisfaction. These variables include age, sex, ethnicity, marital or partnership status, social isolation, subjective health, education, employment, an index of material wellbeing known as the Economic Living Standards Index, and income. The form of these variables will briefly be covered here.

The age variable in the NZGSS consists of 15 age groups, and has been labelled 'agegrp'. Each age group up to the $14^{\text {th }}$ group spans 5 years, beginning with $15-19$ year olds in the first group, to $80-84$ year olds in the $14^{\text {th }}$ group. The $15^{\text {th }}$ group was for respondents 85 years of age and over. The mean age of the population was the 45-49 year old group. A new variable 'agedecade' was created like in the QoLS, to represent age groups in decades. In 'agedecade', 80+ is the highest group and 'younger than 20' is the lowest. From this agedecade group, the 'agetri' three-category variable was created for the analysis of The Altruism Hypothesis. The younger group here is 'Younger than 30 ' and the oldest is ' $60+$ '.

Two dummy ethnicity variables have been created for use in the NZGSS analysis. The first variable indicates individuals who identify as New Zealand European (labelled 'nzeuro'). The second variable represents those who identify as Māori (labelled 'maori'). A value of ' 1 ' on either variable indicates that the respondent picked that ethnicity. In 2008, 7,017 people identify as New Zealand European (80.70\%), in 2010 and 2012 the numbers are 6,861 (80.48\%) and 6,477 (76.91\%) respectively. In 2008, 973 people identify as Māori (11.19\%), in 2010 and 2012 the numbers are 944 $(11.07 \%)$ and $1,108(13.11 \%)$ respectively. Bear in mind that these ethnicity variables are not mutually exclusive, as an individual can identify as both Māori and New Zealand European. In $20084.41 \%$ of the individuals in the dataset identified as both ethnicities, while in 2010 and 2012 this proportion was similar at $4.21 \%$ and $4.74 \%$ respectively.

In the NZGSS respondents were only given the option of responding with either 'male' or 'female' when asked their sex. As such, the sex variable in the combined NZGSS dataset is a dummy variable. The variable is labelled 'male', with ' 1 ' representing those who identify as male and ' 0 ' representing those who identify as female. As in many surveys, the number of female responses was greater than the number of male responses. In 2008 there are 4,791 females (55.08\%) and 3,907 males 
(44.92\%). In 2010 there are 4,762 females (55.85\%) and 3,765 males (44.15\%), and in 2012 there are 4,738 females (56.12\%) and 3,705 males (43.88\%).

The variable regarding relationship status is not well defined in the NZGSS. It is a binary variable labelled 'partnered' recording the respondents 'social marital status', simply coding whether the individual is "partnered" (' 1 ') or "not partnered" ('0'). Being "partnered" is here interpreted to represent individuals who are living with a partner, but are not necessarily married. The two groups are relatively even in each year, although partnered individuals always outnumber those not partnered. In 2008 there are 4,828 people coded as being partnered (55.51\%), in 2010 there are 4,718 $(55.33 \%)$, and in 2012 there are 4,558 (53.99\%).

Feeling lonely or socially isolated from others can have a severely detrimental impact on an individual's wellbeing. The social isolation variable in the NZGSS is labelled 'isolated', and is measured on a 5-point scale in response to the question "in the last four weeks, how often have you felt isolated from others?". The question remained the same in each year, and was introduced to respondents with the statement "Some people say they feel isolated from the people around them while others say they don't. They might feel isolated even though they see family or friends every day". This introductory statement is important, as it explicitly tells the respondent that the question refers to feelings of isolation from others regardless of physical proximity. Responses were originally coded with ' 11 ' indicating "All of the time", ' 12 ' "Most of the time", '13' "Some of the time", "14' "A little of the time" and ' 15 ' "None of the time". The variable was reverse coded so that a higher value on the 'isolated' variable corresponds to a greater feeling of isolation, with "All the time" now coded as ' 5 ' and "None of the time" coded as ' 1 '. Respondents coded as "Don't know" ('89') were recoded as missing. The mean value of isolation in 2008 is 1.57 , and in 2010 and 2012 are 1.54 and 1.53 respectively, all between "None of the time" and "A little of the time".

Health and wellbeing are inextricably related. As such, subjective health variables in surveys are often included as controls in SWB research. The subjective health variable used in the NZGSS analysis is labelled 'health', and is measured on a 5point scale responding to the question "In general, would you say your health is excellent, very good, good, fair or poor?" The responses were originally coded with "excellent" as ' 11 ' and "poor" as ' 15 ', but were reverse coded for the current research so that a higher subjective health value is associated with better health, meaning that 
"excellent" is now ' 5 ' and "poor" is now ' 1 '. The mean subjective health values in 2008, 2010 and 2012 are 3.61, 3.6 and 3.57 respectively, all between 'good' and 'very good'.

Education can be associated with higher wellbeing, although the strength of this association often disappears after controlling for income. Nonetheless, controlling for the effect of educational attainment on SWB is sensible when the option is available. Within the NZGSS, individuals were asked about their highest qualification. For the current dataset a binary control was created labelled 'colled' representing those who have a university qualification. Those who have a Bachelor's Degree or higher are coded as ' 1 ' whilst those without are coded as ' 0 '. In 2008 , there are 1,742 people with a university qualification (20.04\%), in 2010 there are 1,828 (21.44\%), and in 2012 there are $1,789(21.19 \%)$.

The low wellbeing of the unemployed is a common finding in the SWB literature (Dolan et al., 2008), and so employment status is an important control to include in analysis. The variable representing employment status in the combined NZGSS dataset is labelled 'employment', and consists of three groups. The groups are "Employed" ('1'), "Unemployed" ('2'), and "Not in labour force" ('3'). In this instance those not in the labour force are considered to be people who are unemployed but not looking for work, such as retirees and stay-at-home parents. Over the three years, we see a rise in the proportion of respondents who are unemployed or no longer in the labour force, and a fall in those currently employed. In 2008 there are 5,559 people coded as employed (63.96\%), only 246 are coded as unemployed (2.83\%), and 2,886 are coded as not in the labour force (33.21\%). In 2010, there are 5,261 people employed (61.80\%), 315 unemployed (3.70\%), and 2,937 (34.50\%), while in 2012 the equivalent values are 5,145 (60.97\%), 333 (3.95\%), and 2,960 (35.08\%). The 26 individuals across the years who were coded as being of uncertain labour force status ('77') have been recoded as missing data.

The Economic Living Standards Index (ELSI) is a complex measure of the material wellbeing of an individual or household. ELSI addresses the physical circumstances in which people live, the goods and services that they can access, and the economic resources that they have. Rather than focusing specifically on deprivation, ELSI was developed to be a measure that captures information on all of the population 
149 | P a g e

in a more comprehensive manner than simply income ${ }^{33}$. Each of the individual questions that make up the ELSI are provided for respondents to fill out in the NZGSS. As there are so many questions making up the ELSI, there is not the space available here to go into depth on each one. An ELSI score on a scale from zero to 31 is provided as a separate variable (named 'elsi'), and it is this variable which will be used in the analysis. A higher score on the ELSI variable is associated with a better economic standard of living. The mean ELSI score in each year was similar: 22.96, 22.84, and 22.74 in 2008, 2010 and 2012 respectively.

The final control variable is the income variable. Unfortunately, personal income was only recorded in the NZGSS in terms of income groups, rather than actual income. These groups vary in the amount of income that they cover from year to year, reducing the ease with which they could be compared, and further reducing the usefulness of the variable. The coding of the income variable itself changed between 2008 and 2010, but remained the same between 2010 and 2012. As such, the income variable was recoded so that all groups were consistent across the three years. After recoding the combined NZGSS contained an income variable labelled 'income', with 13 distinct income groups. These income groups began with '0" "Loss/Zero" and ' 1 ' “\$1-\$5,000”, and up until group ' 8 ' "\$35,001-\$40,000” rose in increments of $\$ 5,000$. Group ' 9 ' contained those earning " $\$ 40,001-\$ 70,000$ ", group ' 10 ' those earning “\$70,001-\$100,000", group '11' those earning “ $\$ 100,001-\$ 150,000 "$, with the final income group (' 12 ') containing those earning " $\$ 150,000$ or more". The mean income group in 2008 is 6.05 , in 2010 is 6.02 , and in 2012 is 6.12, all roughly corresponding to an income between $\$ 25,001$ and $\$ 30,000$ per year. While the income group variable in the NZGSS is not as useful as an absolute numerical variable would be, it will still suffice for at least a basic control of the relationship between SWB and personal income.

\section{A.3: Te Kupenga}

Te Kupenga was conducted between June and August of 2013, and collected responses from 5,549 Māori in New Zealand. The survey's primary goal was to understand how Māori engaged with Te Ao Māori and how that related to the wellbeing of Māori and their whānau.

\footnotetext{
${ }^{33}$ For more information on ELSI, visit http://www.msd.govt.nz/about-msd-and-our-work/publicationsresources/monitoring/living-standards/living-standards-elsi.html
} 


\section{The Target Population}

The target population valid to participate in the survey included all usually resident individuals who identified as Māori ethnicity or of Māori descent in the 2013 census. Respondents were required to be 15 years of age or older on census day $\left(5^{\text {th }}\right.$ March 2013).

\section{Data Collection Methodology and Reliability of Data}

Like the NZGSS, interviews with respondents were conducted using computerassisted personal interviews (CAPI), lasting an average of 40 minutes. Respondents had the option of completing the survey either in English or te reo Māori. Of the 5,549 interviews, only 27 were completed in either Te Reo Māori or a combination of Te Reo Māori and English. As such, the majority of responses will have been completed in the same language, reducing the likelihood of potential issues that may result from the translation of terms relevant to wellbeing.

As a post-census survey, Te Kupenga represented a unique opportunity to run a comprehensive study of a relatively small sub-group of the population. Using the 2013 census collection period to collect Te Kupenga responses at the same time provided a degree of population coverage not achievable using any other method, and also allowed for responses in Te Kupenga to be linked to census data to create a richer final dataset. The response rate for Te Kupenga was $74 \%$.

\section{$\underline{\text { Variables for Analysis }}$}

As with the NZGSS and the QoLS, the data in Te Kupenga was collected and coded by researchers from a different organisation. While Te Kupenga still required a great deal of data cleaning and exploration before the dataset was ready for analysis, the process was simpler than for the other two surveys, as Te Kupenga only consisted of one year of data, as opposed to three and four for the NZGSS and QoLS respectively. Again, the variables used in this research are a combination of those already available in the dataset, and some newly generated. This sub-section of the chapter will discuss the variables that will be used later in the analysis.

\section{Original Dataset Variables}

The SWB variable of interest within Te Kupenga is again life satisfaction. However, while the life satisfaction measure was very similar across the NZGSS and 
the QoLS, the measure in Te Kupenga has one key point of difference. Both the NZGSS and the QoLS measured life satisfaction on a 5-point scale, while Te Kupenga measures life satisfaction on a much more robust 11-point scale. The question was preceded by "First of all, I am going to ask you a very general question about your life as a whole these days. This includes all areas of your life." While the question itself asked "Where zero is completely dissatisfied, and ten is completely satisfied, how do you feel about your life as a whole?", respondents had the option of selecting a number between ' 0 ' ("Completely dissatisfied") and '10' (Completely satisfied). The life satisfaction variable did not require recoding of values, and is labelled 'lifesatisfaction'. Mean life satisfaction in the entirety of Te Kupenga is 7.744. A total of 7 respondents did not answer the life satisfaction question.

As with the previous chapters, the geographic variables and controlling variables will be covered in a latter part of this sub-section. However, I will first cover a few of the variables within Te Kupenga that are directly relevant to at least one of my hypotheses.

There are eight binary 'unpaid work' variables in Te Kupenga that can be used to test altruism. These eight variables identify whether individuals have participated in a variety of activities helping others within pay in the previous four weeks. For each of the eight variables, those who did take part in the activity in the past four weeks are coded ' 1 ', and those who did not are coded ' 0 '. Questions all start with "in the last four weeks...". Each question is long, and for the purposes of saving space will be summarised. The first question asks about help provided through or for 'a marae, hapu or iwi' (labelled 'unpaidmarae' in the dataset), the second about help provided through or for 'a school, church, sports club, or other group or organisation ('unpaidschool'), the third asks whether they looked after a child under 14 in their household ('unpaidchildinhouse'), the fourth asks if they looked after someone over 14 in the same household 'who needed special care because of illness, disability or old age' ('unpaidadultinhouse'). The following two repeated the previous two for children or adults outside of their household ('unpaidchildouthouse' and 'unpaidadultouthouse' respectively). The seventh question asks if they helped with 'cooking, cleaning, gardening, doing repairs, or any other housework for someone who doesn't live with you' ('unpaidhouseworkouthh'), and the final question asks about other unpaid work ('unpaidother'). 
Social trust questions are used in a wide variety of international surveys. However, Te Kupenga is the only survey in this thesis which includes a variable recording how much individuals trust others. Ability to trust others is often used as a control in SWB research, with people who are more trusting generally also reporting higher wellbeing. Furthermore, SWB research has used social trust as a measure of faith in others to be fair, making it a suitable measure with which to test The Fairness Hypothesis (Delhey \& Dragolov, 2014). The social trust variable is labelled 'trust', and is measured on an 11-point Likert scale. Survey participants were informed "I am now going to ask you a very general question about trust", followed by the question "Where zero is people can never be trusted, and ten is people can always be trusted, how much of the time do you think most people in New Zealand can be trusted?'. The coding of the variable was not altered from the original dataset, with ' 0 ' remaining equivalent to "People can never be trusted", and '10' remaining "People can always be trusted". The mean value of 5.87 in the dataset indicates that many respondents are undecided about the trustworthiness of others, but are slightly more inclined to trust others rather than not. The spread of responses to this variable are quite wide, with a standard deviation of 1.98. For analysis of the fairness hypothesis, binary labelled 'trustbinary' was created from 'trust', with values from ' 0 ' to ' 5 ' recoded as ' 0 ', and ' 6 ' to ' 10 ' recoded as ' 1 '. $58.10 \%$ of the dataset believes others can generally be trusted.

Te Kupenga follows up the previous question on social trust with a number of questions about how much the respondents trust various New Zealand institutions to treat people fairly. While the format of the questions is similar to the perceptions of fairness questions used in the NZGSS, the institutions asked about are slightly altered, and respondents answered the questions on a different scale. Respondents were introduced to the questions with "The next questions are about whether you have trust in various institutions in New Zealand", followed by the question itself: "Where 0 is the public institution can never be trusted, and 10 is the public institution can always be trusted, how much do you trust the < specific institution/system > to treat people fairly?". The words "<specific institution/system>" were replaced with "health system", "system of government", "education system", "police", “courts" and "media". As a result, the question was asked six times about six different institutions, with the respondent ranking each one on the 11-point scale. 
In order to create a singular variable representing an individual's perceptions of fairness in society, the average response of the individual to all six of the questions was taken. In order to generate this variable, "don't know" ('89') responses were recoded to be "neither agree nor disagree" (' 5 ') as this was the response that was most often statistically not significantly different from "don't know". The new variable is labelled 'avgtrusttobefair', and has a mean value almost exactly in the middle at 5.504. A binary labelled 'avgtrustbinary' was created from 'avgtrusttobefair', with values from ' 0 ' to ' 5.49 ' recoded as ' 0 ', and ' 5.5 ' to ' 10 ' recoded as ' 1 '. $55.46 \%$ of the dataset perceives society to be fair.

\section{$\underline{\text { Generated Variables }}$}

I will first address the geographic variables in Te Kupenga, before going on to discuss the SWB inequality variables generated from them.

As Te Kupenga is a survey created by Statistics New Zealand, the geographic variables available after the data was confidentialised are very similar in style to those in the NZGSS. Te Kupenga provides information on the region that the respondents are from, as well as providing a measure of how urban or rural the respondent's location is. As in the NZGSS, these variables are labelled 'region' and 'urban' respectively.

The variable 'region' again consists of six categories of regions around New Zealand. The regions are "North of North Island", "Auckland Region", "Middle North Island", "Wellington Region", "Canterbury Region" and "Rest of South Island". The 'North of North Island' region excludes those living in the Auckland Region. Of the 5,548 people in the dataset, 5, 535 are associated with a particular region. The 'North of North Island group has the highest number of responses with 1,844, while the 'Canterbury Region' group has the fewest with only 374 responses.

The 'urban' variable in Te Kupenga consists only of three categories in contrast to the four in the NZGSS. Respondents are identified as living in either a "Main Urban", "Other Urban", or "Rural" area. 5,546 respondents have been allocated a value within the 'urban' variable, with $64.24 \%$ of those living in a main urban area, $22.45 \%$ living in an other urban area, and the remaining $13.31 \%$ living in a rural area.

While both 'region' and 'urban' are useful variables individually, we will follow the same process used in the NZGSS here by combining the two to create a more specific geographic variable. The 'group' command was used in Stata to combine the 
urban and region variables, creating a new variable called 'reg1'. The new variable 'reg1' now has 18 distinct geographic areas, providing more observations for analysis (see Table 7.1).

As in the NZGSS, the variable 'reg1' was used to create a SWB inequality variable by acting as the grouping for standard deviations in life satisfaction. The 'collapse' command was used to create a dataset of 18 observations based on the 'reg1' groups, each with a mean and standard deviation life satisfaction value. A $19^{\text {th }}$ group was created containing those who were not associated with a region or urban value, and has been dropped from the analysis. Table 7.1 displays the mean life satisfaction values and standard deviation life satisfaction values (in brackets) for each group, rounded to $3 \mathrm{dp}$. The same process was followed when conducting analysis at the regional level, except using only the 'region' variable to group values.

When using the individual scale data, the process for generating the life satisfaction inequality variable in Te Kupenga was the same as that used for both the NZGSS and the QoLS. The 'egen' command was used in conjunction with the categories created by reg1, to create the new standard deviation of life satisfaction variable 'sdreg1'. The 15 individuals who were not associated with a 'region' and an 'urban' variable were dropped from the dataset.

For specific analyses the same processes were used to generate life satisfaction inequality using the 'region' variable instead of the 'reg1' variable. When using 'region' in the collapsed dataset the mean life satisfaction variable is labelled 'meanlsregion' and the standard deviation life satisfaction variable is labelled 'sdlsregion'. In the individual dataset the regional standard deviation life satisfaction variable is labelled 'sdregion'.

Te Kupenga also contains data on how deprived the area that an individual lives in is. This variable was labelled 'NZDep2013' in the original dataset, but has been renamed 'deprivation' in the current dataset for ease of understanding. 'NZDep2013' in Te Kupenga stands for 'New Zealand Deprivation 2013', and provides small area indexes of socioeconomic deprivation in New Zealand ${ }^{34}$. The New Zealand deprivation index indicates the deprivation of a meshblock (a small geographic unit) on a scale from

\footnotetext{
${ }^{34}$ For more information on the New Zealand Deprivation Index and it's measurement, see http://www.otago.ac.nz/wellington/otago069936.pdf
} 
$\mathbf{1 5 5} \mid \mathrm{P}$ a g e

' 1 ' (least deprived) to '10' (most deprived). The mean deprivation score across the dataset is 7.013, indicating that most Māori who responded to Te Kupenga live in high deprivation areas.

\section{Controlling Variables}

Numerous other variables were included from the original data in order to control for known factors that may have an influence on life satisfaction, and to allow for analysis of the wellbeing and wellbeing inequality relationship by group. These variables include age, sex, marital or partnership status, loneliness, subjective health, smoking status (as a measure of objective health), employment, and income. The form of these variables will be briefly summarised in this sub-section.

As in the NZGSS and the QoLS in the chapters before this, age is recorded in Te Kupenga in the form of age groups. Respondents gave their exact age in the survey, which was then converted into an age group variable for the CURFs provided to researchers. The age group variable is labelled 'agegrp' in the dataset, and consists of seven distinct groups. The groups begin with those aged "15-19" ('1') and "20-24" ('2'), before increasing in increments of 10 years in each group from " $25-34$ " ('3') to "55-64" ('6'), and ending with the "65+" ('7') category. The mean age group in the combined dataset after dropping the 15 missing observations is 3.93 , close to the " 35 44 " year old age group.

The sex variable in Te Kupenga (labelled 'sex') is once again a binary variable, and is derived from the respondent's linked census data. Consistent with the previous surveys, in the Te Kupenga dataset sex was coded with ' 1 ' indicating a "Male" respondent, and ' 0 ' indicating a "Female" respondent. After dropping the 15 missing observations, there were 3,091 respondents who identified as female (55.86\%), and 2,442 who identified as male $(44.14 \%)$.

Even though Te Kupenga is a survey of Māori wellbeing, respondents still had an option of choosing an ethnicity. Many people identify as more than one ethnicity, and so despite all respondents in the survey being of Māori descent, a number of other ethnic identities were chosen. Interestingly, there were also a number of respondents who did not choose Māori as an ethnicity, in spite of being of Māori descent. The most common ethnicity chosen was Māori, followed by New Zealand European (NZ European). As a result of numerous privileges, NZ Europeans generally fare better on 
a number of outcomes relevant to wellbeing in comparison to Māori. It is therefore possible that those individuals of Māori descent who also identify as NZ European would fare better than those who do not identify as NZ European. Another possibility is that those who do not consider themselves Māori at all would fare better in terms of wellbeing than certain groups of Māori. As such, dummy variables for both NZ European and Māori were created in order to control for any effects that race may have upon SWB. The NZ European variable is labelled 'nzeuro'. 3,012 respondents (54.44\%) do identify as NZ European, and are coded as ' 1 ', while 2,521 respondents (45.56\%) do not identify as NZ European, and are coded ' 0 '. The Māori ethnicity dummy is labelled 'maori'. The values are labelled ' 1 ' for those who do identify as Māori, and ' 0 ' for those who do not. A total of 4,993 individuals identify as Māori (90.24\%), while 540 do not (9.76\%). As the sample of individuals who do not identify as Māori (but still have Māori ancestry) is so small, they not separated in the analysis. 2,551 individuals identify as both Māori and NZ European, while 76 individuals identify as neither (fitting into the 'other' category).

Partnership status is also measured within Te Kupenga. Labelled 'partnered' in the dataset, the variable is a simple binary variable which indicates whether an individual is "Partnered" (' 1 ') or "Not Partnered" (' 0 '). The survey population is roughly equally divided between partnered and non-partnered individuals, with 2,757 people coded as partnered (51.27\%) and 2,620 people coded as not partnered (48.73\%). In Te Kupenga, partnership status is not asked directly within the survey, and instead is derived from census data. As in the previous surveys, being 'partnered' here is interpreted as being in a long term relationship with another individual (i.e. not restricted to just those who are married).

The loneliness question in Te Kupenga is structured much like the isolation question in the NZGSS. The question is prefaced with "People who have a lot of contact with family and friends can still sometimes feel lonely, while those that have little contact with others might not feel lonely at all.", followed by the question "In the last four weeks, how much of the time would you say you have felt lonely?". The variable is labelled 'loneliness' and was initially coded with "all of the time" as ' 11 ' and "none of the time" as ' 15 ', but were reverse coded for the current research, meaning that "all of the time" is now ' 5 ' and "none of the time" is now ' 1 '. 
Subjective health in Te Kupenga is labelled 'health', and is measured on a 5 point scale responding to the same question as used in the NZGSS: "In general, would you say your health is excellent, very good, good, fair or poor?". Identical to the NZGSS dataset, the variable was initially coded with "excellent" as ' 11 ' and "poor" as ' 15 ', but were reverse coded for the current research, meaning that "excellent" is now ' 5 ' and "poor" is now ' 1 '. The mean subjective health value in the combined dataset is 3.48 , between 'good' and 'very good'.

The variable measuring employment status in Te Kupenga again assigns individuals to one of three categories. These categories are labelled "Employed" ('1'), "Unemployed" ('2'), and "Not in Labour Force” ('3'). The employment status variable itself is labelled 'employment'. Of the 5,498 individuals who responded with an employment status in Te Kupenga, 3,538 are coded as employed (64.28\%), 339 are coded as unemployed (6.17\%), and 1,625 are coded as not in the labour force (29.56\%).

Income is measured in income groups within Te Kupenga. 5,168 individuals in the dataset are coded into an income group. Income was not asked directly in Te Kupenga, instead being garnered from the respondents answer to the income group question in the census. Within the Te Kupenga CURF, eight unique income groups are recorded. The lowest group is "Loss/Zero" ('0’), followed by “ $\$ 1-\$ 10,000$ ” (' 1 '), after which income groups rise by $\$ 10,000$ per group until reaching “ $\$ 50,001-\$ 70,000$ ” ('6”) and " $\$ 70,000$ and above" ('7'). The income group variable is labelled 'incomepersonal' in Te Kupenga. The mean income group in the data is 3.2 , roughly equivalent to the “\$20,001-\$30,000” group. 